\title{
VERIFICATION OF Business Rules Programs
}

\author{
Bruno Berstel - DA Silva
}

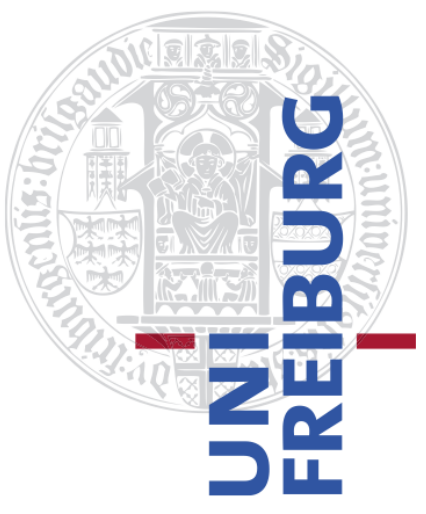

Dissertation

ZUR ERlangung DES DOKTORGRAdes

DER TEChNiSChEN FAKULtÄT

Der Albert-Ludwigs-Universität Freiburg

Defended on July 11, 2012 
Bruno Berstel-Da Silva

Verification of Business Rules Programs

Version 1.1

Built on October 17, 2012

Dekan:

Erstgutachter:

Zweitgutachter:

Vorsitz:

Beisitz:
Prof. Dr. Bernd Becker

Prof. Dr. Andreas Podelski

Prof. Dr. Georg Lausen

Prof. Dr. Bernhard Nebel

Prof. Dr. Peter Thiemann 
To Emil Post, to Robert Floyd, and to all the zebras around the world. 



\section{Zusammenfassung}

Der Beitrag dieser Arbeit ist der (unserem Wissen nach) erste formale Ansatz zur Verifikation von Geschäftsregel-Programmen. Wir schlagen eine Verifikationsmethode vor, um die Korrektheit von Geschäftsregel-Programmen kompositionell zu beweisen. Der Ansatz ermöglicht Autoren von Geschäftsregeln und Entwicklern einer Werkzeugumgebung, Eigenschaften der Ausführung von Geschäftsregel-Programmen zu verstehen, formal zu beschreiben und zu beweisen. Der übergeordnete Beitrag der Arbeit ist, die logischen Grundlagen bereit zu stellen, um Geschäftsregeln überhaupt erst als Gegenstand der wissenschaftlichen Untersuchung in Semantik und Verifikation einführen zu können.

Schichtenarchitekturen benutzten in wachsendem Maße die sogenannten Geschäftsregeln, um die Logikschicht (auch: Businessschicht) zu kodieren. Die Erfassung und die Ausführung von Geschäftsregel-Programmen wird durch ein Geschäftsregel-Managementsystem (GRMS) unterstützt. Ein Geschäftsregel-Programm besteht aus einer Menge von voneinander unabhängigen Regeln, d.h., bedingten Zuweisungsinstruktionen, die in einem modularen, Fall-basierten Ansatz gesammelt werden. Ein Geschäftsregel-Programm ist deklarativ in dem Sinne, dass es nicht den Kontrollfluss spezifiziert; die Regeln werden auf der gegebenen Menge von Objekten ausgeführt, nach und nach für jede Regel und jedes Objekt, in nicht-festgelegter Reihenfolge.

Die wissenschaftliche Forschung hat sich bisher auf die Effizienz der Ausführung von Geschäftsregel-Programmen durch ein GRMS konzentriert. Eine Vielfalt von Kompilierungs- und Ausführungsschemas sind entwickelt worden, so insbesondere der bekannte Rete-Algorithmus. Die Verifikation von Geschäftsregel-Programmen wurde bisher als Gegenstand wissenschaftlicher Forschung vernachlässigt. Der Bedarf an Korrektheit ist jedoch für Geschäftsregel-Programme nicht weniger einleuchtend als für sicherheitskritische Systeme, auch wenn die auf die Spiel stehenden Risiken ökonomisch sind, und üblicherweise nicht lebensbedrohlich.

Die Arbeit besteht aus drei Hauptteilen.

Im ersten Teil präsentieren wir eine Formalisierung des Ausführungsverhaltens von Geschäftsregel-Programmen. Bisher exisistierende Beschreibungen hingen inhärent von den Eigenheiten des jeweiligen Kompilierungsschemas ab. Die Aufgabe, das Ausführungsverhalten eines Geschäftsregel-Programms formal und allgemein zu erfassen, wird erschwert durch die schiere Vielfalt der Kompilierungs- und Ausführungsschemas, die in existierenden GRMS benutzt werden. Wer haben ein allgemeines und gleichzeit formal einfaches Rahmenwerk entworfen, das es uns in die Lage versetzt, das Ausführungsverhalten von Geschäftsregel-Programmen zu beschreiben und die Hauptunterschiede zwischen den verschiedenen Ausführungsschemas herauszustellen. Die dank der Formalisierung des Ausführungsverhalten von Geschäftsregel-Programmen mögliche präzise Beobachtungsweise führt zu der Erkenntnis, dass die Einfachheit von Geschäftsregeln nur oberflächig gilt. Tatsächlich kann das Zwischenspiel von Ausführungen von einer oder mehreren Regeln auf einem oder mehreren, möglicherweise geteilten Objekten (die nicht-deterministisch aus einer endlich, aber unbeschränkt großen Menge ausgewählt werden) extrem komplex sein, und dies schon für kleine Beispiele.

Eine Ausführung ist formal eine Folge von Zuständen. Um die Unbeschränkt- 
heit der Größe der Objekt-Mengen in den jeweiligen Zuständen berücksichtigen zu können, modellieren wir einen Zustand als eine Struktur der Logik erster Stufe. Wir können der Vielfalt der Ausführungsschemas (die neuen Alternativen zum Rete-Algorithmus eingeschlossen) Rechnung tragen, indem wir Konzepte einführen, die es uns erlauben, zwischen der Anwendbarkeit und der Auswählbarkeit einer Regel zu unterscheiden.

Im zweiten Teil der Arbeit führen wir Korrektheitsspezifikationen für Geschäftsregel-Programmen ein. Bisher bestand die einzige Möglichkeit, die Korrektheit eines Geschäftsregel-Programms zu beurteilen, darin, jede Regel des Programms einzeln zu betrachten und ihre Anwendung auf einzelne Objekte im Hinblick auf mögliche Verhaltensmuster zu untersuchen. Es war nicht möglich, den globalen Effekt der Anwendung eines ganzen Geschäftsregel-Programms auf eine Objekt-Menge zu erfassen.

Die Schwierigkeit, formal die Korrektheit von Geschäftsregel-Programmen zu definieren, stammt aus der Diskrepanz zwischen dem lokalen Verhalten während der Ausführung einer Regel-Anwendung und dem globalen Effekt des gesamten Geschäftsregel-Programms. Das lokale Verhalten bezieht sich nur auf das Objekt, auf das die Regel angewandt wird; der globale Effekt bezieht sich auf die gesamte, also endliche aber unbeschränkte Menge von Objekten, auf der das Programm ausgeführt wird.

Wir definieren die Bedeutung eines Hoare-Tripels für ein Programm und globale Zusicherungen als eine konservative Erweiterung der Bedeutung eines Hoare-Tripels für eine einzelne Regel-Anwendung und lokale Zusicherungen. Wir erhalten so Korrektheitsspezifikationen, die dem modularen und dem deklarativen, d.h., Kontroll-unspezifischen Aufbau von Geschäftsregel-Programmen gerecht werden.

Im dritten und letzten Teil der Arbeit führen wir eine kompositionelle Verifikationsmethode für Geschäftsregel-Programme ein. Die Schwierigkeit bei der Kompositionalität stammt aus den möglichen Interferenzen zwischen RegelAnwendungen während der Ausführung eines Programms. Daher kann der Beweis einer Korrektheitseigenschaft für ein Geschäftsregel-Programm nicht einfach der Zerlegung des Programms in seine syntaktischen Bestandteile, also der Regeln, folgen. Geleitet von der Intuition hinter der Owicki-Gries-Methode für parallele Programme, präsentieren wir ein Beweissystem mit einem erweiterten, auf Geschäftsregeln angepassten Begriff der Kompositionalität.

In dem Beweissystem kann ein globales Hoare-Tripel für ein Programm von den lokalen Hoare-Tripeln für dessen einzelne Regeln abgeleitet werden. Wir beweisen, dass das Beweissystem 'sound' und relativ vollständig ist (wir benutzen relative Vollständigkeit in dem gleichen Sinn wie bei Hoare-Logik). Wir leiten Beweisregeln für wichtige Klassen von Geschäftsregel-Programmen und von Zusicherungen als Spezialfälle der allgemeinen Beweisregel ab. Wir benutzen verschiedene Beispiele, um die praktische Anwendung der allgemeinen Beweisregel und ihrer Spezialfälle zu veranschaulichen.

In einem nicht-technischen Anhang zu dieser Arbeit demonstrieren wir das praktische Potential unseres Ansatzes im Kontext eines existierenden kommerziellen Geschäftsregel-Managementsystems. Dieses GRMS verfügt über ein leichtgewichtiges Analyse-Modul (unter dem Namen "Rule Static Analysis"). Wir zeigen, dass den verschiedenen Analyse- und Verifikationsfunktionalitäten des GRMS dank des in dieser Arbeit vorgestellten Ansatzes zur formalen Verifikation eine solide Fundierung gegeben werden kann. 


\section{Summary}

The technical contribution of the thesis is to present - to the best of our knowledge, for the first time - an approach to the formal verification of business rules programs. We propose a verification method for proving correctness properties for a business rules program in a compositional way. The approach enables rule authors and tool developers to understand, express formally, and prove, properties of the execution behavior of business rules programs. The conceptual contribution of this thesis is to present the enabling framework for treating business rules as a topic of scientific investigation in semantics and program verification.

Multitier architectures increasingly use business rules to encode the application tier (the so-called business logic). The authoring and the execution of business rules programs is supported by a Business Rules Management System (BRMS). A business rules program consists in a set of mutually independent rules, that is, conditional update statements authored in a modular, case-bycase approach. A business rules program is declarative in that it does not specify the control flow; the set of rules are executed on a set of objects, exhaustively for each rule and each object, in any order.

Until now, the emphasis in research has been on optimizing the efficient execution of business rules programs by a BRMS. A variety of compilation and execution schemes have been developed, including the well-known Rete algorithm. The verification of business rules programs has been neglected as a topic of scientific research. The need for correctness is, however, no less obvious for business rules programs than it is for safety-critical systems, even though the risks at stake are economic and usually not life-threatening.

The thesis is structured in three main parts.

In the first part, we present a formal definition of the execution behavior of business rules programs. Previous descriptions of business rules execution depended on the intrinsics of a specific compilation scheme. The very first issue in the formalization task is the diversity of compilation and execution schemes used in existing BRMS. We have designed a general, yet formally simple, framework that enables us to describe the execution behavior of business rules programs and to single out the main differences between the various execution schemes. The formalization of the execution behavior of business rules programs allowed us to observe that the apparent simplicity of business rules is only superficial. Indeed, the interplay between executions of one or several rules on one or several, possibly shared, objects (selected nondeterministically from a finite, but unbounded set) can become extremely complex, even for small examples.

An execution is formally a sequence of states. To account for the unboundedness of the set of objects in each state, we model a state as a first-order logic structure. We account for the diversity of execution schemes, including recent alternatives to the Rete algorithm, by introducing concepts that allow us to distinguish between the applicability and the eligibility of a rule.

In the second part of the thesis, we introduce correctness specifications for business rules programs. Previously, the only way to assess the correctness of a business rules program was to examine each of its rules and its possible behaviors when applied to various objects. The expected global effect of a program had to be expressed in natural language, which could result in misunderstandings 
between authors of programs and their users. The difficulty in formally defining the correctness of business rules programs stems from the gap between the local behavior of the single application of a rule and the global effect of a whole business rules program on a set of objects. The local behavior involves only the object on which the rule is applied, whereas the global behavior involves the whole, finite but unbounded, set of objects on which the program is run. We define the meaning of a Hoare triple for a program and global assertions as a conservative extension of the meaning of a Hoare triple for a single rule application and local assertions. We thus obtain correctness specifications that follow the modular and declarative nature of a business rules program.

In the third and final part of the thesis, we propose a compositional verification method for business rules programs. The challenge of compositionality stems from the possibility of interference between rule applications during the execution of a program. Proving a correctness property of a business rules program cannot simply follow the decomposition of the program into its syntactic constituents, i.e., the rules. Borrowing the intuition of the Owicki-Gries method for parallel programs, we present a proof system that features an extended notion of compositionality, suitable for business rules programs. By our proof system, a global Hoare triple for a program can be derived from local Hoare triples for its individual rules. We show that the proof system is sound and relatively complete (we use relative completeness in the same sense as for Hoare logic). We derive proof rules for important classes of business rules programs and assertions, as special cases of the general proof rule. We use several examples to illustrate the practical application of the general proof rule and its specializations.

In a non-technical appendix to the thesis, we demonstrate the practical potential of our formal approach in the context of an existing commercial Business Rules Management System. This BRMS comes with a lightweight analysis engine (named "Rule Static Analysis"). We show that its various analysis and verification features can be given a solid foundation thanks to the approach to the formal verification of business rules programs presented in this thesis. 


\section{Acknowledgments}

I carried out this thesis as a remote-working student, first of the Max-Planck Institut für Informatik of Saarbrücken, then of the Institut für Informatik of the Albert-Ludwigs-Universität in Freiburg. At the same time I was a software engineer at ILOG, and then at IBM after this company acquired ILOG. My thanks go to Nitsan Seniak for proposing me the subject of rule program verification within ILOG; to Hassan Aït-Kaci for establishing the connection with Andreas Podelski; and to Andreas Podelski, my supervisor, for offering me to reify our thoughts as a $\mathrm{PhD}$ thesis, and for his support and guidance throughout the years. It is also an honor for me to count Prof. Lausen as the Zweitgutachter, as well as Prof. Nebel and Prof. Thiemann as the members of my defense committee.

Achieving the present work in an industrial environment would not have been possible without the initial agreement of Jean-François Abramatic, and overall the creativity in management demonstrated by Nitsan Seniak and Antoine Melki. More generally, I would like to thank the members of the "BEAR R\&D Governance" team within ILOG for their patience.

Substantial advances in this work have been achieved during a six months period spent in the Modélisation et Vérification team of the LIAFA laboratory in Paris Diderot university. I am most grateful to Ahmed Bouajjani for welcoming me. This stay was a major step for me, thanks to the friendly atmosphere and stimulating discussions. My thoughts go to Mihaela Sighireanu, Tayssir Touili, Cezara Drăgoi, Constantin Enea, Mathilde Bouvel, and Ahmed Rezine, as well as the whole "Vérif" team.

Within ILOG I have also benefited from enriching conversations and exchanges. I was nurtured by the outcomes of the RVS project, which included Hassan Aït-Kaci, Ulrich Junker, Michel Leconte, and Andreas Podelski; by the proofreading and most relevant comments of Hassan, Michel, Philippe Bonnard, and Hélène Kencker; and by the fruitful discussions with Hugues Citeau, Amina Chniti, Aurélie Baton, Marie Girard, and Philippe Laborie. Very special thanks are deserved by Michel for his continuous support, sustained stimulation, and for passing down the demand for accuracy inherited from my father.

During the long process that this work has represented for me, the right words were found on several occasions by Ulrich Junker and Claire David to help me find the required energy.

Last, but far from least, I am specially grateful to my wife and children for their support and their care. 



\section{Contents}

Acknowledgments vii

1 Introduction 1

1.1 Business Rules Management Systems . . . . . . . . . . . . . . . 1

1.1.1 From Business Policies to Business Rules . . . . . . . . . 1

1.1.2 A Brief Genealogy of Business Rules Management Systems 2

1.1.3 Industrial Context . . . . . . . . . . . . . . . 3

1.2 Motivation .......................... 4

1.3 Summary of Contribution . . . . . . . . . . . . . 7

1.4 Thesis Outline ................... 8

2 Related Work 11

2.1 Rule-Based Paradigms: Model vs. State . . . . . . . . . . . . . . 11

2.2 Formalization and Verification of Rule Programs . . . . . . . . . 14

2.3 Verification of Pointer and Concurrent Programs . . . . . . . . . 16

$\begin{array}{lll}\text { I Rule Programs } & 19\end{array}$

3 Syntax of Rules and Rule Programs 21

3.1 Signature ... . . . . . . . . . . . . . . . 21

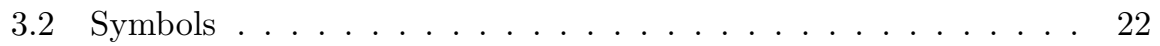

3.3 Expressions and Formulas . . . . . . . . . . . . . . 23

3.3.1 Parameterized Languages of Expressions and Formulas . . 23

3.3.2 Well-Typed Expressions and Formulas . . . . . . . . . . 25

3.3.3 Formulas Are Flat . . . . . . . . . . . . . . . . 26

3.3.4 Theory Used in Examples . . . . . . . . . . . . . . 26

3.4 Assignment . . . . . . . . . . . . . . . . . . 27

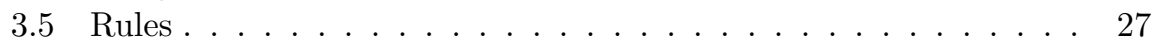

3.5.1 Rule Variables . . . . . . . . . . . . . . . . . . 28

3.5 .2 Rule Guard . . . . . . . . . . . . . . . . . . . . 29

3.5.3 Rule Action . . . . . . . . . . . . . . . . . . . . 29

3.6 Rule Programs . . . . . . . . . . . . . . . . 30

4 States and State Assertions 31

4.1 States Are First-Order Logic Structures . . . . . . . . . . . . . . 32

4.1 .1 Domain .................. . . 32

4.1.2 Variable Valuations .............. 32 
4.1.3 Interpretation of Expressions . . . . . . . . . . . . . . 33

4.1.4 Interpretation of Formulas . . . . . . . . . . . . . . 34

4.1.5 States . . . . . . . . . . . . . . 37

4.2 State Assertions . . . . . . . . . . . . . . . . . . . . 37

4.2.1 Assertions . . . . . . . . . . . . . . . 38

4.2.2 Global Assertions . . . . . . . . . . . . . . . . . . . . . 38

4.2.3 Assertions Focused on a Rule . . . . . . . . . . . . 39

4.3 Transition Assertions . . . . . . . . . . . . . . . . . . . . . 39

4.3.1 Forward Transition Assertions . . . . . . . . . . . . . . 39

4.3.2 Backward Transition Assertions . . . . . . . . . . . . . 40

4.3.3 Global Transition Assertions . . . . . . . . . . . . . . 41

4.4 Semantics of Assignment . . . . . . . . . . . . . . . . . . . . . . 41

4.4.1 Update of an Attribute . . . . . . . . . . . . . . 41

4.4.2 Executing an Assignment . . . . . . . . . . . . . . 42

4.4.3 Assignment as a Relation Between States . . . . . . . . . 43

4.5 Rule Guard and Action . . . . . . . . . . . . . . . . . . . 43

4.5.1 Rule Guard . . . . . . . . . . . . . . . . . . 43

4.5.2 Rule Action . . . . . . . . . . . . . . . . . 44

5 Operational Semantics of Rule Programs $\quad \mathbf{4 5}$

5.1 Rule Program Execution, Informally . . . . . . . . . . . . . . . . 45

5.2 Working Memory . . . . . . . . . . . . . . . . . . . 48

5.2.1 Type-System Compliant States . . . . . . . . . . . . . . . . 49

5.2 .2 Objects as Instances of Types . . . . . . . . . . . . . . . . . 50

5.2.3 Preservation Properties ............. 51

5.3 Rule Execution . . . . . . . . . . . . . . . . . . . . 52

5.3 .1 Rule Instance . . . . . . . . . . . . . . . 52

5.3 .2 Applicability of a Rule Instance . . . . . . . . . . . . . 53

5.3.3 Application of a Rule Instance . . . . . . . . . . . . . . 53

5.3.4 Execution of a Rule Instance . . . . . . . . . . . . . 55

5.3.5 Executions of a Rule . . . . . . . . . . . . . . 55

5.4 Rule Program Execution . . . . . . . . . . . . . . . . . . 57

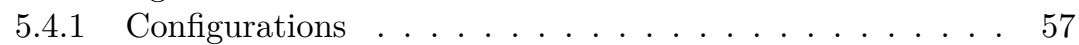

5.4 .2 Initial Configuration . . . . . . . . . . . . . . 57

5.4.3 Transition Between Configurations . . . . . . . . . . . 58

5.4.4 Executions of a Rule Program . . . . . . . . . . . . . 59

5.5 Selection Strategies . . . . . . . . . . . . . . . . . 61

5.6 Eligibility Strategies . . . . . . . . . . . . . . . . 62

5.6 .1 Identity Eligibility Strategy . . . . . . . . . . . . . 63

5.6 .2 Refraction Eligibility Strategy . . . . . . . . . . . . 64

5.6.3 Sequential Execution Strategy . . . . . . . . . . . . 67

5.6.4 One-Shot Eligibility Strategy . . . . . . . . . . . . 68

II A Hoare Logic for Rule Programs $\quad 71$

6 Correctness of Rule Programs $\quad 73$

6.1 Preliminaries ........................ 73

6.1.1 Fixing the Execution Strategy . . . . . . . . . . . 73

6.1.2 Correctness Formulas, Proofs, Proof Systems . . . . . . . 74 
6.2 Correctness Formula for a Single Rule . . . . . . . . . . . . 75

6.3 Rules Compared to Conditional Statements . . . . . . . . . . 78

6.3 .1 Loop-Free Programs . . . . . . . . . . . . . . . . . 78

6.3.2 Rules vs. Conditional Statements . . . . . . . . . . . . 80

6.4 Correctness Formula for a Rule Program . . . . . . . . . . . . 81

6.4.1 Syntax and Semantics . . . . . . . . . . . . . . . 82

6.4.2 From Rules to Rule Programs . . . . . . . . . . . . . . 83

7 Correctness of Programs: A Comparison $\quad 87$

7.1 Correctness of Loop-Free Parallel Programs . . . . . . . . . . . 87

7.1.1 Loop-Free Parallel Programs . . . . . . . . . . . . . . 88

7.1.2 Loop-Free Parallel Program Derived from a Rule Program 90

7.1.3 Rule Programs vs. Loop-Free Parallel Programs . . . . . . . 91

7.2 Correctness of Parallel Programs _ . . . . . . . . . . . . . . 92

7.2 .1 Ghost Variables . . . . . . . . . . . . . . . . . . . . . 93

7.2 .2 while Programs . . . . . . . . . . . . . . . . 94

7.2 .3 Parallel Programs . . . . . . . . . . . . . . . . . 95

7.2.4 Parallel Program Derived from a Rule Program . . . . . . 96

7.2.5 Rule Programs vs. Parallel Programs . . . . . . . . . . . . 99

7.3 Correctness of Nondeterministic Programs . . . . . . . . . . . 101

7.3.1 Nondeterministic Programs . . . . . . . . . . . . . . . 101

7.3.2 Nondeterministic Program Derived from a Rule Program 103

III Proof Rules for Rule Programs 107

8 Main Steps in Rule Program Verification 109

8.1 Interference Cases in Rule Programs . . . . . . . . . . . 110

8.1.1 Cross-Rule Interference . . . . . . . . . . . . . . . . 110

8.1 .2 Interference due to Aliasing . . . . . . . . . . . . . 111

8.2 Unary Rules . . . . . . . . . . . . . . . . . . . . . . . . . 112

8.3 A First Proof Rule . . . . . . . . . . . . . . . . . . . . . . 114

8.4 Usage . . . . . . . . . . . . . . . . . . . . 118

8.4.1 Use the Proof Rule Bottom-Up . . . . . . . . . . . . . . 119

8.4 .2 What to Look for . . . . . . . . . . . . . . . . . 119

8.4.3 Application of the Verification Method . . . . . . . . . 120

8.5 Incompleteness . . . . . . . . . . . . . . . . . . 122

9 A Verification Method for Rule Programs 125

9.1 Taking Eligibility into Account . . . . . . . . . . . . 125

9.1.1 Eligibility Ghost Variables . . . . . . . . . . . . . . . 126

9.1.2 Eligibility-Aware Assertions . . . . . . . . . . . . . . 127

9.1.3 Eligibility-Aware Execution . . . . . . . . . . . . . . 128

9.1.4 Eligibility-Aware Correctness Formulas . . . . . . . . . . 131

9.2 Interference Freedom . . . . . . . . . . . . . . . . . 133

9.2 .1 Cross-Rule Interference . . . . . . . . . . . . . . . . . . 133

9.2 .2 Interference due to Aliasing . . . . . . . . . . . . . . 134

9.3 General Proof Rule . . . . . . . . . . . . . . . . . . . . . . . 135

9.4 Relative Completeness . . . . . . . . . . . . . . . . . . . . 139

9.5 Application of the Verification Method . . . . . . . . . . 146 
9.5.1 Approach . . . . . . . . . . . . . . . . . . . 146

$9.5 .2 \quad$ Example . . . . . . . . . . . . . . . . . . . . . 148

10 Specialized Proof Rules 157

10.1 Eligibility-Aware Unary Rules . . . . . . . . . . . . . . . . . 157

10.1.1 Proof Rule . . . . . . . . . . . . . . . . . . 158

10.1 .2 Example . . . . . . . . . . . . . . . . . 160

10.2 Aliasing-Free N-ary Rule Programs . . . . . . . . . . . . . . . 164

10.3 Disjointness in Rule Programs . . . . . . . . . . . . . . . . . 167

10.3.1 Disjoint Unary Rules . . . . . . . . . . . . . . . . . . . 167

10.3.2 Disjoint Rule Programs . . . . . . . . . . . . . . . . 170

11 Conclusion and Future Work

173

A Verification in an Industrial Business Rules Management System 179

A.1 Preliminaries . . . . . . . . . . . . . . . . . . . . . . . 179

A.2 Rule Applicability . . . . . . . . . . . . . . . . . . 181

A.3 Impact Analysis . . . . . . . . . . . . . . . . . . 184

A.4 Redundancy . . . . . . . . . . . . . . . . . . . . 187

A.5 Confluence and Completeness . . . . . . . . . . . . . . . . . . 189

A.6 Safety . . . . . . . . . . . . . . . . . . . . . . . . 191

A.7 Discussion . . . . . . . . . . . . . . . . . . . . . . 192

$\begin{array}{ll}\text { List of Figures } & 195\end{array}$

$\begin{array}{ll}\text { List of Proof Rules } & 197\end{array}$

$\begin{array}{ll}\text { Bibliography } & 199\end{array}$

$\begin{array}{ll}\text { Index } & 215\end{array}$ 


\section{Chapter 1}

\section{Introduction}

Our focus in this thesis is on business rules programs, that is, rule programs as handled in Business Rules Management Systems. We define Business Rules Management Systems (BRMS) in this chapter, and we position them among other rule-based paradigms in the next one.

The conceptual contribution of this thesis is to present the foundation for treating business rules as a topic of scientific investigation in semantics and program verification.

The technical contribution of the thesis is to present an approach to the formal verification of business rules programs. We propose a verification method for proving correctness properties for a business rules program in a compositional way. Compositionality means that the proof of a correctness property for a program is built up from correctness properties for the individual rules. With our approach, we aim at enabling rule authors and tool developers to understand, express formally, and prove, properties of the execution behavior of business rules programs.

In the present chapter we settle the context and motivation of our work. We then give a short description of the subsequent chapters.

\subsection{Business Rules Management Systems}

\subsubsection{From Business Policies to Business Rules}

The business policies that describe how a company, small or large, should be run in order to execute its mission take various forms, from informal manuals to methodologies, to standards. For a few decades now, they have often been automated as software to be executed by a computer. Software business applications in such areas as industry, finance, insurance, or administration implement the policies of an enterprise or organization for such tasks as loan approval, pricing, claim processing, and many more.

Change in general, and in business policies in particular, is inevitable. It has become increasingly frequent due to an acceleration in regulations and business. Organizations have to conform with more and more regulations, such as new accounting standards, laws addressing environmental concerns, or related to global or homeland security, etc. In addition, competition and market demand 
motivate changes in the business policies of a company, by introducing new pricing plans, implementing new production schemes, etc.

Due to the stress put by these changes on software systems and I.T. departments, the traditional, monolithic implementation of a business application, in which business policies are hard-coded in databases or in COBOL, is no longer able to deliver the agility required. This is where Business Rules Management Systems come into play.

To cope with frequent change and achieve the desired agility, the part of the application that implements the business policies is logically externalized as business rules. That is, it is reimplemented (possibly semi-automatically from its original implementation) as an external component, by means of constructs such as condition-action rules or decision tables. The business application is equipped with a rule engine responsible for executing this new component. Also, tools are provided for business users to manage the business rules, such as versioning or collaborative working features. Additional tools allow the I.T. department to devise semi-natural, domain-specific rule languages, so that business experts with no advanced computer knowledge can read and even write business rules.

A Business Rules Management System (BRMS) is a software system that gathers these authoring, management, and execution tools. Using business rules and a BRMS to implement the business logic of an application is a recognized way to achieve greater agility [MS06]: the business policies are no longer hardcoded in the application, and business experts, who own the knowledge about the business policies, can author business rules directly.

\subsubsection{A Brief Genealogy of Business Rules Management Systems}

Business Rules Management Systems, or BRMS, can be seen as the convergence of production systems and business rules.

Production systems were originally proposed by logician Emil Post in 1943 [Urq09] in his works on decidability. They were brought to computer science by Robert Floyd in 1961 [Knu03, Flo61] as a way to describe translation from one language to another, a seminal step in compiler construction. They really became a reasoning tool, as well as a programming one, in the work of Allan Newell on problem solving [NS72]. Newell was designing heuristics inspired by human reasoning. In these heuristics, operators were applied to a state to evolve it towards a goal. He resorted to production systems when he equipped each operator with a description of the conditions under which the operator could be applied, actually turning the operators into if-then statements [Pic07].

Such if-then statements, initially named productions and later rules, became an ubiquitous tool in artificial intelligence and beyond. Section 2.1 compares rules in BRMS (and hence production rules) with some other occurrences of the concept of rule in computer science.

A production system is thus composed of: a set of production rules that describes the operations at hand and the conditions under which they can be applied; a working memory that models the state operated upon by the production rules; and an inference engine responsible for choosing production rules and applying them to the working memory. Production systems, and more generally expert systems, gave birth to numerous implementations, the seminal one being OPS5 [For81, BFKM85], based on the Rete algorithm invented by Charles Forgy 
[For82]. The other historical algorithm is TREAT [Mir87]; the Gator algorithm [HH93] is derived from both of them. The key ideas in the Rete algorithm are to compile the production rules into a network where the predicates shared by the rules are not duplicated, and to use this network to maintain incremental information on the applicability of each rule on the working memory. This algorithm and its variants are still in use in many production rules engines.

Recently, the W3C has issued the RIF-PRD recommendation [dSMHP10]. This recommendation is part of the Rule Interchange Format effort. It proposes a standard for a Production Rule Dialect, with an abstract syntax and an operational semantics. Although the door is left open for alternatives, the recommendation describes the semantics of Rete only.

The other parent of BRMS, business rules, appeared in the 1970s as a tool to model the business policies introduced in the previous section. Although Ronald Ross claims to be "the father of business rules", a number of other authors contributed to the definition and expanded use of business rules as an approach to representing business knowledge [Ros03, vH02, vHG06]. Methodologies have been defined and a consortium, the Business Rules Group, has been created [2].

Users of business rules, seeing them as a knowledge representation tool, are more interested in the elicitation and harvesting of business expertise than in the automation of their execution. Software tools around business rules aim at assisting business analysts and knowledge engineers in defining glossaries of business terms, authoring business rules, and checking the consistency of rule bases. A connection with the semantic web has been established through the definition of standards such as the Object Management Group's one on Semantics of Business Vocabularies and Business Rules (SBVR) [OMG08].

Business Rules Management Systems have inherited from the business rules community the objective of remaining close to the business expert. The vocabulary definition and authoring tools found in BRMS reflect this concern. From production systems they have inherited the operational orientation towards execution of the rules. The execution components of BRMS provide this aspect by being integrated in applications, in which production rules implement the business logic.

\subsubsection{Industrial Context}

The commercial successors of OPS5 as production system implementations, such as CLIPS [CR03], Jess [FH03], Rules [Alb94], or Nexpert [ALS90], were designed for the implementation of complex algorithms using production rules. Typical examples are found in telecommunications, with alarm correlation applications [HSV99], where signals from physical sources are entered in working memory, and higher-level events are synthesized and correlated by the rules, to eventually issue alarms to the operator. In such rule programs, executions often include the chaining of several rules, and the system state permanently evolves as new signals are inserted.

However, with the increasing demand for agile software systems to cope with frequent changes in business policies, production systems evolved to meet business rules and were renamed Business Rules Engines [TR07]. As part of this evolution, the kind of rule programs that were written changed as well. The number of rules in a rule program increased, especially since they often resulted from the automated translation of existing databases or COBOL components, 
but their complexity decreased. Executions now more rarely include complex inferences and the combination of numerous rules. They rather involve a handful of objects and act as a procedure call, than perform an endless loop on a flow of data.

This evolution motivated the introduction in Business Rules Engines of alternatives to the historical Rete-like algorithms, such as the sequential execution mode described in Section 5.6.3 [IBM11, Red10, Fai07]. It also fostered the development of authoring and management tools, including web-based tools directly targeted at the business user. All in all, this evolution defined a new family of industrial products that took the name of Business Rules Management Systems [MS06, RG08].

Analysts have valued the BRMS market in 2008 at $\$ 285 \mathrm{M}$, with an estimated $10 \%$ growth until 2013 [Hen09]. Both the size of the market and the added value of BRMS have led large software editors to acquire the leading BRMS vendors or products. Nowadays, the main players in the BRMS arena are:

- IBM [5] with the IBM Websphere Operational Decision Management line of products, after the acquisition of ILOG;

- FICO [4] with the FICO ${ }^{\mathrm{TM}}$ Blaze Advisor $®$ product, after the acquisition of Blaze, formerly Nexpert [ALS90];

- Oracle [8] with the Oracle Business Rules product, after the acquisition of Haley;

- Red Hat [9] with the JBoss Enterprise BRMS product, repackaged from the Drools open-source project;

- SAP [11] with the NetWeaver Business Rules Management product, after the acquisition of Yasu;

- Corticon [3] with the Corticon Business Rule Management System product.

With the incorporation of BRMS into broader catalogues of software products, a trend develops, in addition to the market for a pure BRMS offer, to integrate them with Business Process Management or Predictive Analytics software.

\subsection{Motivation}

As exposed in the previous section, Business Rules Management Systems are increasingly used in industrial applications to implement fast-changing, missioncritical parts of their application tier - the so-called business logic. A business rules program consists in a set of mutually independent rules, that is, conditional update statements authored in a modular, case-by-case approach. A business rules program is declarative in that it does not specify the control flow; the set of rules are executed on a set of objects, exhaustively for each rule and each object, in any order.

In spite of the crucial role of business rules programs, the emphasis in research on rule programs has until now been on optimizing their execution by a BRMS. A variety of compilation and execution schemes have been developed, including the well-known Rete algorithm. The verification of business rules programs has been neglected as a topic of scientific research. This may be due to 
their categorization as information systems, that is, rather as a business area than as computer science. The need for verification tools in BRMS has only recently been expressed [Hen06]. It is, however, no less obvious for business rules programs than it is for safety-critical systems, even though the risks at stake are economic and usually not life-threatening. Fulfilling this need requires to describe the correctness of a rule program in BRMS, and hence to introduce a formalization of these programs.

\section{The Need for Formalization}

Business Rules Management Systems are industrial products: as such they are ultimately defined by their implementations. Yet, of course, reasoning in a rigorous manner about a business rules program, for example to verify its correctness, requires a formal description of the language and of the execution engine. More generally, the task of providing a formal basis for verification features in a BRMS is a cornerstone to an agreement on their definitions, for example between product marketing and development teams.

Proposing a formalism for an industrial product is a trade-off between the simplifications required to achieve a practical model, and keeping the substance of the modeled product. In particular, such a formalism must take into account the fact that rules in a BRMS are most of the time based on an object-oriented model, defined by the application in which they are integrated. For example, it should be able to handle a rule such as the one given in Fig. 1.1, which was written with IBM Websphere Operational Decision Management.

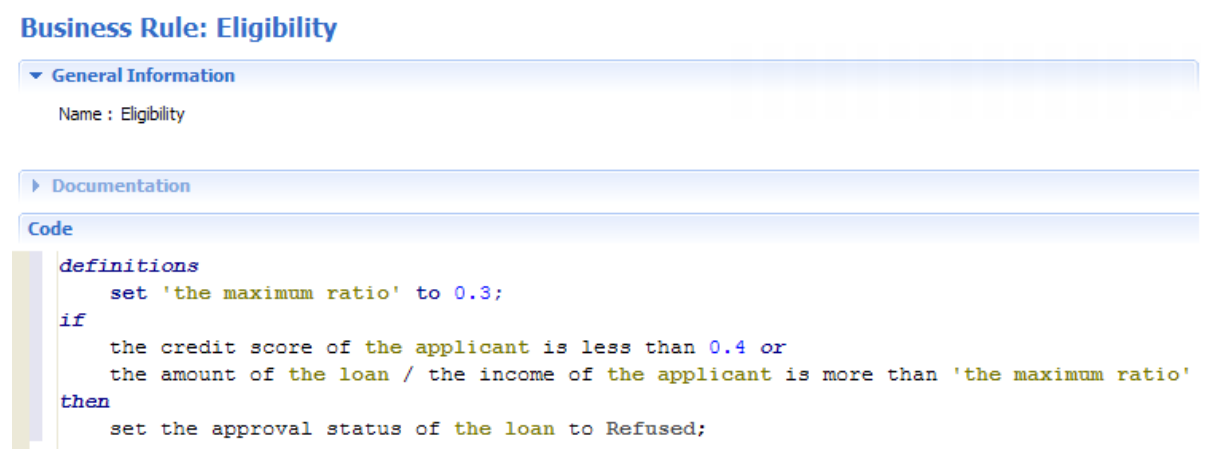

Figure 1.1: A business rule written with IBM Websphere Operational Decision Management.

In Part I, we present a formal framework based on first-order logic structures. Although not exotic, this framework meets the above requirements. In addition, it supports the description of the recently introduced alternatives to the Rete algorithm.

As a result, our formalization of rule programs makes it possible to specify the expected behavior of a business rules program, and to verify (that is, to prove formally) that the executions of the program will comply with this specification. This goes further than most verification features of current BRMS products, 
which either are only based on a syntactic analysis of the rules, or are limited to the execution of individual rules and do not consider the full execution of a rule program.

Note also that apart from enabling formal verification, being able to express specifications can be useful in other cases. A well-known example is testing, that is, running the program on some input data and checking that the actual output conforms to the one predicted by the specification. In addition to indicating the outputs expected, a specification can be used to assess the comprehensiveness of the input data sets used for tests with respect to the data space that the program might receive as input. The specification can also be used to enhance it by generating missing test cases.

Other usages of specifications include run-time testing, that is, instrumenting the executable code to trigger an exception when a specification is violated; and historical testing, that is, comparing recorded executions of the program with specifications, for example to detect changes in the program behavior over time or to assess the compliance of the program in its current implementation with respect to a proposed evolution of its specification.

\section{The Need for Verification}

The need for verification of programs developed with a Business Rules Management System (BRMS) comes both from reasons that apply to all software systems, and from reasons more specific to BRMS themselves.

As highlighted earlier in this chapter, one major role of a BRMS in industrial applications is to provide tools for the authoring and execution of programs written as rules. In other words, it provides a programming language and its interpreter. As such, the question of the correctness of the programs with respect to a specification is natural (or should be). This concern is reinforced by two factors.

Firstly, the functions implemented with a BRMS are often critical functions for the company. These can range from stock trading to loan approval, to production control, etc. A failure in a business-critical application could have dramatic consequences.

Secondly, these applications represent large software projects, both by the size of the teams involved in their development and maintenance, and by the number of rules that a BRMS-based application often contains. Indeed, it is common to find applications involving thousands of rules [SBJ87, 6]. As mentioned previously, this often comes from the fact that a BRMS-based application is the result of the externalization of a component previously implemented in COBOL or with databases. It is usually considered simpler and safer to mechanically translate the policies encoded in database table rows into as many rules, than to reengineer the implementation into a more compact but more subtle set of rules.

As a consequence, the development and maintenance of a BRMS-based application will benefit from tooling dedicated to verification, as for any software project with a large code base.

In addition to the above, organizations have felt the need to monitor the agility that they have gained from the use of a Business Rules Management System. Indeed a BRMS gives business users access to the rules. This feature is motivated by the idea that business users, as domain experts, have a good 
knowledge of what the business rules should be. It increases the agility of the overall application by shortening its production cycle, thus allowing the organization as a whole to adapt to change faster.

Yet this increased flexibility gained by giving programming power to users with no advanced qualification in computer programming demands some control. In early BRMS products, the lack of verification features has been pointed as a shortcoming [Hen06]. As an answer the initial argument of agility has evolved into that of controlled agility, where program analysis techniques are applied to rule programs to try and raise alarms when relevant. A sampling of such rule analysis features contributing to controlled agility is given in Appendix A.

\subsection{Summary of Contribution}

The contribution of this thesis consists in a formal, logic-based description of the behavior of rule programs in Business Rules Management Systems (BRMS), and in the introduction of a Hoare logic and a verification method for these rule programs. With this, we provide tools to express and verify global correctness properties on rule programs, based on local knowledge about the individual rules in the program.

As a first step, we present a formal characterization of the execution behavior of business rules programs. The task of formally characterizing the execution behavior of business rules programs faces several issues. A very first issue is the diversity of compilation and execution schemes used in existing BRMS. Previous descriptions of business rules execution depended on the intrinsics of each specific compilation scheme. We have designed a general, yet formally simple, framework that enables us to describe the execution behavior of business rules programs and to single out the main differences between the various execution schemes. The formalization of the execution behavior of business rules programs allowed us to observe that the apparent simplicity of business rules is only superficial. Indeed, the interplay between executions of one or several rules on one or several, possibly shared, objects (selected nondeterministically from a finite, but unbounded set) can become extremely complex, even for small examples.

An execution is formally a sequence of states. To account for the unboundedness of the set of objects in each state, we model a state as a first-order logic structure. We account for the diversity of execution schemes, including recent alternatives to the Rete algorithm, by introducing concepts that allow us to distinguish between the applicability and the eligibility of a rule.

Based on our formal framework, we introduce correctness specifications for business rules programs. Previously, the only way to assess the correctness of a business rules program was to examine each of its rules and its possible behaviors when applied to various objects. The expected global effect of a program had to be expressed in natural language, which could result in misunderstandings between authors of programs and their users. The difficulty in formally defining the correctness of business rules programs stems from the gap between the local behavior of the single application of a rule and the global effect of a whole business rules program on a set of objects. The local behavior involves only the object on which the rule is applied, whereas the global behavior involves the whole, finite but unbounded, set of objects on which the program is run.

We define the meaning of a correctness formula of the form $\{p\} \mathcal{R}\{q\}$ where 
$\mathcal{R}$ is a rule program, as a conservative extension of the meaning of a correctness formula of the form $\{p\} r\{q\}$ where $r$ is a rule. We thus obtain correctness specifications that follow the modular and declarative nature of a business rules program. With correctness formulas for both rules and rule programs, we give the BRMS user a tool for expressing knowledge about rules and specifications of rule programs. We also define the building blocks of a compositional verification method for rule programs.

Finally, we propose such a compositional verification method for rule programs. The challenge of compositionality stems from the possibility of interference between rule applications during the execution of a program. Proving a correctness property of a business rules program cannot simply follow the decomposition of the program into its syntactic constituents, i.e., the rules. Borrowing the intuition of the Owicki-Gries method for parallel programs (see [OG76], but also the presentation of proof systems for sequential and concurrent programs in [AO97] by Krzysztof Apt and Ernst-Rüdiger Olderog, and the revised version [AdBO09] by Apt, Olderog, and Frank de Boer), we present a proof system that features an extended notion of compositionality, suitable for business rules programs. By our proof system, a global correctness formula for a program can be derived from local correctness formulas for its individual rules. We show that the proof system is sound and relatively complete (we use relative completeness in the same sense as for Hoare logic). We also study several particular cases of this proof rule for specialized classes of rule programs and correctness formulas.

\subsection{Thesis Outline}

After the bird-eye view of Business Rules Management Systems given in the first section of this chapter, Chapter 2 relates them to other bodies of work. In that chapter, we see that rule programs as handled by BRMS have both similarities to, and differences from, other paradigms that use the concept of rules. From the viewpoint of their execution, and therefore of verifying execution properties, they also share features with databases, programs with pointers, and concurrent programs.

The subsequent eight chapters contain our contribution and are structured in three parts. Part I (Chapters 3 to 5) presents our formalization of rule programs as handled by BRMS. Part II (Chapters 6 and 7 ) defines a Hoare logic for these rule programs. Part III (Chapters 8 to 10) proposes a compositional verification method for rule programs. The focus of each of these chapters is as follows.

Chapters 3 and 4 define the syntax and semantic of a prototypical rule language, as well as assertions to express conditions on the states of systems that execute programs written in this rule language. Such programs can handle objects, reason on the values of their attributes, and perform updates on these attributes. The business facet is a parameter to this language, embodied in a theory and a type system that are considered fixed orthogonally to the rule language. The theory describes the data types (such as numbers) and the operations allowed on them; the type system structures the objects into classes.

Chapter 5 defines the meaning of putting rules together as a rule program, and executing a rule program on a set of objects. We describe the operational semantics of a rule program by modeling a rule engine with an abstract machine. 
This abstract machine is parameterized by execution strategies, which represent the varying aspects among the algorithms used in BRMS, either Rete or not. We give a formal description of the main execution strategies, including the oneshot strategy. Parts II and III study rule programs from the viewpoint of this execution strategy, as it is a reasonable approximation of the Rete algorithm and a good abstraction of the principal Rete alternative, in the sense that it abstracts away its implementation-dependent aspects.

Chapter 6 studies the semantics of rule programs from the viewpoint of the relation between their inputs and their outputs. To this end it defines correctness formulas in the classical form of so-called Hoare triples $\{p\} r\{q\}$, where $r$ is a rule. It then defines correctness formulas of the same form $\{p\} \mathcal{R}\{q\}$, where $\mathcal{R}$ now is a rule program.

Chapter 7 builds on the definition of correctness formulas for rule programs, to compare rule programs with parallel and then nondeterministic programs. This comparison investigates whether a parallel program, and then a nondeterministic one, can be found for any rule program, so that it has the same input/output behavior. We will see that in general, one cannot find a parallel program with the same input/output behavior as a rule program. This result motivates the search for a verification method to derive a correctness formula for a rule program from individual correctness formulas for its rules.

Chapters 8 to 10 propose several proof rules achieving the above, under various conditions. In Chapter 8 we first introduce a simpler proof rule for a particular class of rule programs, so as to exhibit the core mechanism at play. We then expose in Chapter 9 the general case and prove that the corresponding proof rule is sound and relatively complete. Chapter 10 completes the presentation of proof rules with several other particular cases that can benefit from specialized proof rules.

Chapter 11 concludes with a summary of the work exposed in this thesis. In this last chapter we also suggest directions for extensions of this work.

Finally, in Appendix A, we demonstrate the practical potential of our formal approach in the context of an existing commercial Business Rules Management System. This BRMS developed by IBM comes with a lightweight analysis engine. Its various analysis and verification features are not an application of the present work, as they are limited to the executions of individual rules, as opposed to considering the full execution of a rule program. However, we show that they can be given a solid foundation thanks to the approach to the formal verification of business rules programs presented in this thesis.

Across the chapters of Parts I to III, we start each section with an abstract of its content. This abstract is typeset in a specific way, and is intended to allow the knowledgeable reader to skip the section without losing the continuity of the discussion. As a consequence, the abstracts might contain forward references to concepts introduced in the section. An abstract should therefore not be seen as an introduction to the section, at the risk of finding it redundant with the section body. 


\section{Chapter 2}

\section{Related Work}

Rule programs as handled by Business Rules Management Systems (BRMS) are the focus of this thesis. In the present chapter we relate these programs to other programming paradigms, whether rule-based or not. We concentrate on the similarities and differences that have an impact on our objective, which is to help understand the effects, and prove correctness properties on the executions of rule programs as handled by BRMS.

The first section of this chapter reviews several rule-based paradigms, which allows us to relate them more or less tightly to rule programs in BRMS. Then we discuss some formalization and verification approaches of related rule-based paradigms, but also of concurrent programs.

\subsection{Rule-Based Paradigms: Model vs. State}

Rule programs as handled by Business Rules Management Systems are by far not the only occurrence of the concept of rules in computer science. To sort out the numerous paradigms that give an important role to rules, we adopt a schematic classification between those where rules are used to model the knowledge about a given domain, and those where they are used to perform a computation. We refer to the former as model-oriented rule-based paradigms, and to the latter as state-oriented ones.

This classification, which follows a similar one proposed by Victor Vianu [Via97], has the advantage of concentrating on the intent behind using rules. This in contrast, for example, with a common distinction based on whether the rules are used in backward- or forward-chaining. In the latter classification, the focus is on the way in which rules are put into action: this gives little, if any, information on their actual role.

The distinction between model- and state-oriented rule-based paradigms should not hide the fact that the usages of rules are not clear cut. In particular, and as suggested by their genealogy briefly presented in Section 1.1.2, BRMS inherit from paradigms in both categories.

We conclude this section with a brief review of some other cases where the two categories are combined. 


\subsubsection{Model-Oriented Rules}

In rule-based paradigms to which we refer as model-oriented, the intent is to build a description of a given body of knowledge. Rules are used to provide parts of this model intensionally. They are commonly gathered in rule bases, in contrast with state-oriented paradigms which often collect them in rule programs.

Model-oriented rules are of course abundantly used in the large area of artificial intelligence that studies knowledge representation; see for example [LN94] or [Sow99] for a survey. Deriving from this area are business rules in the sense of the description given in Section 1.1.2, which are used to implement models of business policies.

As a consequence, Business Rules Management Systems naturally inherit from these rules. This inheritance materializes primarily in the authoring components. By authoring components, we mean the tools that provide business experts with rule languages that are as close as possible to their domain-specific vocabulary. BRMS will for example use standards such as the OMG one on Semantics of Business Vocabularies and Business Rules (SBVR) [OMG08].

Another important occurrence of model-oriented rules is to be found in deductive databases, or deductive rules in databases [Min96, Lud98]. Here also rules are used to provide parts of the model intensionally. Examples are rules used to enrich the data stored in the base (for instance through recursive views), or rules used to specify integrity constraints in a concise way.

The increasing work on the semantic web [BLH01] in the last decade has leveraged several concepts from artificial intelligence, with a renewed interest for ontologies. An extensive survey of the combination of rules and ontologies in the context of the semantic web is given in $\left[\mathrm{ADG}^{+} 05\right]$. This remains an area of active research with an annual Conference on Web Reasoning and Rule Systems [CL08]. As mentioned in $\left[\mathrm{ADG}^{+}\right.$05, p. 20], the kind of rules that are considered are model-oriented ones.

It is interesting to note also that the semantic web community is showing interest [DL07] in the SBVR standard, which had initially been promoted by the business rules community.

\subsubsection{State-Oriented Rules}

In rule-based paradigms to which we refer as state-oriented, the role of rules is not to build a model intensionally for a body of knowledge, but rather to solve a problem. In this, rules are used to evolve a state of the system towards a goal, by performing a series of computations. Particularly in this category of rule-based paradigms, both forward- and backward-chaining approaches are represented.

Again, artificial intelligence provides several occurrences of state-oriented rules. As mentioned in Chapter 1, production systems were introduced through the work of Alan Newell on heuristics reproducing the human approach [Pic07, NS72]. Business Rules Management Systems have inherited from production systems the basis for their execution components: the engines that are in charge of selecting the rules to apply, and actually perform their actions on the current state of the system.

The most obvious contribution is the Rete algorithm [For82], the variants and 
evolutions of which are implemented in the rule engines of BRMS. This influence of production systems on BRMS is the one that is the most relevant to us, since we are interested in the execution of rule programs as handled by Business Rules Management Systems. Despite the seminal role of Rete, alternatives to Rete-based execution have emerged in the last decade [IBM11, Red10, Fai07]. However, in Chapter 5 we exhibit a structure in the execution of rule programs that is common to all execution algorithms. We achieve this by dissociating applicability and eligibility of rules, and exploit it to define a semantics of rule programs formally.

Databases, which as just seen use model-oriented rules through deductive rules, also use state-oriented rules in active databases [WC96]. While the rules in production systems are also called condition-action rules, the active rules in databases are often called event-condition-action rules, because they react to events that are internal or external to the database. The actions taken by these rules range from checking integrity constraints to updating data in the base, to triggering broader operations outside of the database. Even when the purpose of these rules is to ensure the integrity of the database, the fact that they do so by applying updates to the base gives them a state orientation.

The use of event-condition-action rules, not in the context of databases but in that of the semantic web, has been investigated in $\left[\mathrm{BFM}^{+} 06, \mathrm{BBB}^{+} 07\right]$.

\subsubsection{Combining Both Approaches}

Although the classification between model- and state-oriented rules reflects an actual difference in the intent for using rules, there are several cases where a combination of the two approaches has been investigated.

One of them is automated planning and scheduling, another occurrence of rules in artificial intelligence. In this field, rules can be used to infer additional information on a given state from domain knowledge; this usage of rules is called axiomatic inference and is rather model-oriented. Rules are also extensively used in the state space approach to planning, to evolve the state of the system towards a goal, that is, with a state orientation. See [GNT04] for an overview of automated planning and scheduling.

The deductive and active rules in databases give another illustration of the use of either approach in a common area, with deductive rules oriented towards knowledge modeling and active rules oriented towards computation. In [Lud98] their seemingly diverging capabilities are integrated by the introduction of the Statelog language, which Bertram Ludäscher defines as a state-oriented extension of Datalog. This goes further than the work of Vianu, who provides alternate semantics for the same syntax in [Via97]. Indeed, Ludäscher simultaneously puts into play features of both kinds of rules to build Statelog.

The FLORID engine [ $\left.\mathrm{FHK}^{+} 97\right]$ was developed to evaluate rules in deductive databases, thus in a model-oriented usage of rules. In [SSL07] the use of the Rete algorithm, which was introduced for the implementation of production rule systems, is investigated as an evaluation technique.

Another example of combining the two approaches is given by the work on performing rule-based computations on the semantic web, that is, on universes described by ontologies. This usage of state-oriented rules on the semantic web is addressed in $\left[\mathrm{ADG}^{+} 05\right]$, and is one of the subjects of study for the REWERSE network [10]. The specific question on how Business Rules Management Systems 
could be enabled to handle data modeled by such intensional mechanisms as ontologies is one objective of the ONTORULE project [7], addressed for example in $\left[\mathrm{HdBR}^{+} 09, \mathrm{CDAC} 10\right]$.

\subsection{Formalization and Verification of Rule Pro- grams}

\subsubsection{Formalization}

Business Rules Management Systems inherit from both model-oriented and state-oriented rules, although the influence of each category expresses itself in distinct components. On the one hand, the model orientation has an influence in the rule authoring components, through the design and implementation of the vocabularies targeted at the business experts. On the other hand, the state orientation shows in execution components, through the semantics given to rules. We can therefore expect that the formalization of rule programs as handled by BRMS will receive an influence of formalisms adopted for either orientation of rule-based paradigms.

Databases also make an extensive use of both kinds of rules with deductive and active rules being the respective representatives of model- and state-oriented rules. The Datalog language [GM77, Ull88, Min96] is at the heart of deductive and active databases, and formalizations of numerous variants of this language have been proposed [AV91, AV88, Via97, Min96]. These formalisms, which provide several semantics for Datalog, are all logic-based.

Among them, F-logic [KL89] has the peculiarity of having originally been developed for deductive databases, and of being used more recently in the context of the semantic web as a formalism for ontologies [AKL09], together with description logic $\left[\mathrm{BCM}^{+} 03\right]$ and order-sorted features logic [AKPCG97, AK07]. There is no definitive formalism for rules in the context of the semantic web. The choice depends primarily on the formalism adopted for ontologies, and on whether the rules are used in a model orientation to provide parts of the knowledge, or in a state orientation to perform computations.

The principal point of effort when formalizing state-oriented rules, such as active rules in databases or production rules, is the fact that they perform updates on states. To this end, [dBR09] uses $\mu$-calculus to represent propositional production systems, and fixed-point logic to represent production systems with variables. This approach leads to using first-order logic structures as we do. A formalization of production systems with situation calculus is given by [BL07], while [BW94] represents active rules in databases by means of an extended relational algebra.

The formalizations proposed by François Fages and Rémi Lissajoux in [FL92], then by Claude Kirchner, Pierre-Étienne Moreau et al. in [CKMM04], are also based on first-order logic. They are restricted to the Rete algorithm. Furthermore, the former explicitly discards the control strategy from its scope, and the latter gives no detail of how it affects the execution of a rule program. On the other hand, the RIF-PRD recommendation by the W3C [dSMHP10] does provide a rigorous description of the control strategy of the Rete algorithm and its influence on rule program execution. 
Our formalization goes one step further towards the verification of properties of rule programs as handled by BRMS, in particular by considering the execution strategy as a parameter (Chapter 5), and by allowing the description of a saturation semantics that extends to parallel or nondeterministic programs (Chapter 7).

\subsubsection{Verification of Model-Oriented Rule Programs}

As seen in Section 2.1.1, model-oriented rules are primarily used in knowledge management applications and in deductive databases. In these contexts the verification concerns are almost exclusively focused on consistency and completeness of the knowledge base, respectively of the database. See [PS94, SSS82, MP05] for a survey of problems and techniques in the verification of knowledge bases, and [vLNPS85] for an illustration on a specific knowledge management system. Deductive databases reuse these techniques, or develop specific ones [KSS87, Min96].

The verification of the consistency of an ontology can be seen as a particular case of consistency of a knowledge base. Specific investigations have been led when ontologies are used in the semantic web context $\left[\mathrm{PGC}^{+} 11\right]$, and in particular when they are implemented with description logic [PHE10].

The verification of business rules in the sense of Section 1.1.2, that is, rules modeling business policies, has been the subject of extensive study by Rik Gerrits and Silvie Spreeuwenberg [GS00]. It has given birth to the commercial product LibRt VALENS, which claims to be used by a few Business Rules Management Systems. However the description of the product in [GS00] and on the company website, furthermore confirmed by an in-depth study from the standpoint of a BRMS [Nak04], shows that LibRt VALENS considers rules with a model orientation, and proves to be out of focus for a BRMS. For example, a self-contradiction is signaled when a rule is not applicable in the state that results from its execution, which is a rather common case in a state-oriented rule applications as they are nonmonotonic. More generally, the verification concerns of LibRt VALENS focus on rule base consistency and completeness, and transpose badly to production rules.

Consistency and completeness of a rule base are not our primary focus here. Rather, we are interested in correctness properties of executions of a rule program. However, we can provide an interpretation of consistency and completeness in the context of Business Rules Management Systems. For example, a rule that can never be applicable, no matter the values of the attributes of the objects in the working memory, can be seen as an inconsistency in the rule program. Additional examples of such interpretations of consistency for rule programs in BRMS are given in Appendix A and in [CAC11, $\left.\mathrm{PGC}^{+} 11\right]$. Completeness can also be relevant for BRMS: checking the completeness of a rule program means verifying whether the program can be executed from all possible working memories - or from working memories in a subspace of interest.

\subsubsection{Verification of State-Oriented Rule Programs}

While the verification concerns for model-oriented rules are focused on consistency and completeness, those for state-oriented rules primarily concentrate on confluence and termination. 
Several approaches to the verification of confluence and termination in active databases are reviewed in [BW94]. Among them, [KU94] reduces eventcondition-action rules to conditional term rewriting, and [HH91] restricts the rule language to guarantee confluence. [BW94] itself proposes an approach based on an extension of the relational algebra. On the other hand, [LL98] focuses on the termination problem in active databases.

The connection between production rules and term-rewriting systems has been explored with the intention of using the techniques of rewriting systems to analyze properties, and eventually verify the correctness, of production systems. In [SS94, SS95, SS96, SS97, SS99], James Schmolze and Wayne Snyder propose a rewrite semantics for OPS5. This proposal includes a form of constrained rewriting for negative tests ("there is no object such that...", which we do not address). In addition, techniques such as Knuth-Bendix completion [KB70] are applied to test the confluence of production systems and repair it in case of failure, and to detect design issues such as redundancy. This approach has shortcomings, which are listed by the authors themselves [SS96, pp. 514515]. Some of them can probably be addressed by appropriate extensions of the formalism: for example the limitation of expressions to Boolean ones, and of actions to additions to, and removals from, the working memory. Other limitations however, such as not modeling the selection and eligibility strategies, may reveal too severe to apply this approach to rule programs as handled by BRMS.

For rule programs as handled by Business Rules Management Systems, confluence can be defined as the property for a rule program that all its executions from a given state lead to the same final state. This property is described in [BL10]. We do not study it here, mainly because general confluence involves several concurrent executions and hence cannot be expressed with correctness formulas of the form $\{p\} \mathcal{R}\{q\}$. However, in Appendix A, we present examples of particular cases of confluence checking.

In addition to confluence and termination verification, analyses have been designed in the context of production systems to find design flaws. For example, [PSM91] describes a method to analyze OPS5 programs statically, in order to detect relations between production rules that would suggest errors in the design of the program.

The verification of safety properties in BRMS rule programs is within our focus. [BL10] addresses it by translating this verification task into a constraint satisfiability problem. However, the technique described in this paper for proving these safety properties lacks compositionality, contrary to the one introduced in Part III.

\subsection{Verification of Pointer and Concurrent Pro- grams}

Verification of programs with a Hoare logic is a widespread technique. Its origins are in the works of Robert Floyd [Flo67] and Tony Hoare [Hoa69]. It has since been extended to numerous programming paradigms. Surveys can be found for example in [dBdBZ80, Apt81, Apt84, Old84].

We have seen that rule programs as handled by Business Rules Management Systems have common points with databases, in particular because produc- 
tion rules can be seen as a special case of event-condition-action rules in active databases, although the execution semantics of either kind of rule programs are different. In addition they share features with two other, non-rule-based paradigms: programs with pointers and concurrent programs.

The similarities with programs with pointers come from the fact that business applications integrating with Business Rules Management Systems are typically object-oriented applications. As a result, rules in BRMS handle objects with attributes and methods. As mentioned in Chapter 1 (and formally described in Chapter 5) the objects on which the rules reason are handled through a set called the working memory.

In many aspects the working memory is similar to a heap: it is finite but not bounded, it is accessed randomly, in particular when rule instances are formed, and it is dynamic in two ways. Firstly, the links between the objects can be updated by the execution of the rules (this aspect is covered in the present work). Secondly, rules can add objects to the working memory or remove objects from it (we do not cover this aspect). In contrast with programs with pointers however, there is usually no concern such as shape analysis when verifying rule programs.

Beyond the similarities between the working memory and a heap, the fact that rules handle objects brings in the aliasing problem, that is, the fact that two distinct variables can hold the same object. This has to be taken into account, in particular, in the way updates of attributes are interpreted (Section 4.4) or rule execution is defined (Section 5.3). We adopt the approach of Joseph Morris [Mor82], which consists in including a conditional expression in the formula that defines the effect of assignment. We present it on page 41; it is also presented in detail in [AdBO09, pp. 42-45].

Rule programs in BRMS also have important similarities with concurrent programs, which is a well-studied topic [Old91a, Old91b, Lam93, OR93, Lam02, BO01]. Clearly the working memory can be seen as a shared memory. Furthermore, the execution of a rule program as described in Chapter 5 shows two degrees of concurrency, which result from the Cartesian product between rules and object tuples that is performed by the rule engine. One way to look at this is to consider each rule in the program as a process, within which all objects are concurrent; the symmetric vision holds as well, where all objects are processes and run the same program made of the rules in concurrence. In addition, both viewpoints must account for the fact that rules do not match individual objects but object tuples, which introduces synchronization constraints. Chapter 7 handles this complexity by transposing the saturated semantics of rule engines onto parallel programs.

In the light of the similarities between rule programs in BRMS and concurrent programs, the question naturally arises of whether the verification methods for concurrent programs could apply to rule programs. Such methods were initially designed by Edward Ashcroft [Ash75] using a global invariant, then independently made compositional by Leslie Lamport [Lam77], and by Susan Owicki and David Gries [OG76]. These methods are also described or discussed in [Lam93, SA86, AdBO09].

We shall see in Chapter 7 that in general, a rule program as handled by a BRMS cannot be simulated by a concurrent program. As a consequence, the verification of a rule program cannot be performed by translating it into a concurrent one and then applying the verification methods for concurrent programs. 


\section{Part I}

\section{Rule Programs}





\section{Chapter 3}

\section{Syntax of Rules and Rule Programs}

The rule programs we consider are written in a language built on a set of symbols. The symbols are assembled into formulas and statements, to eventually form rules and rule programs. The symbols and assembly rules are exposed in this chapter; the next two chapters give the semantic interpretation of elements of rule programs and of rule programs themselves.

\subsection{Signature}

In this section ${ }^{1}$ we introduce the signature on which all the subsequent languages (expressions, formulas...) are defined. The symbols in this signature are introduced in the next section. We also postulate that a theory and a type system are fixed, which represent the business-specific aspects of rule programs, inherited from the BRMS-based application.

We consider a set $\Sigma$ of symbols. This signature includes symbols for constants, variables, types, and attributes, as well as operators and symbols for writing statements.

Rule programs implement the business logic of a wider BRMS-based application, and to this end handle and transform the data of this application. The data types involved can range from Boolean values, to numbers, either integral or real, to enumerations of symbolic values such as colors or customer categories, and beyond. Since we consider rule programs integrated in objectoriented applications, rules will also handle objects, which are basically data structures that aggregate references to other objects and values of the data types just mentioned. Objects are classified by an application into object types, as described below.

\footnotetext{
${ }^{1}$ Let us remind that in the eight central chapters, each section starts with an abstract like this one. These abstracts do not aim at introducing the section, but rather at allowing the knowledgeable reader not to read the entire section. As a consequence, the abstract might contain forward references to, or be redundant with, the section body. Also, the reader can skip the abstract if $\mathrm{s} /$ he intends to read the section.
} 
Data types vary from one BRMS-based application to another. Fundamentally, they are parameters to the rule language. To formalize application data types, we consider from now on that a theory $\Theta$ has been fixed. This theory describes the data types from the application that are handled by the rule language. The theory comes with a signature, which contributes to $\Sigma$ with constant symbols and operators.

In addition, we consider that a type system $\mathcal{T}$, as defined in Section 3.3.2, has been fixed. The type system describes the classification among object types enforced by the BRMS-based application. It contributes to $\Sigma$ with a set of symbols (the type names, defined below) that denote the object types.

In our examples, we will use real numbers and enumerated symbolic values as the data types of our applicative theory. We will also use object types such as persons, shopping carts, or cars.

\subsection{Symbols}

This section defines the symbols found in rules and rule programs. In particular, variable symbols denote variables that will hold objects, and attribute symbols denote the structural features of these objects.

\section{Symbols for constants}

Constant symbols denote the values handled by rule programs. We include the Boolean constants true and false in our rule language. Other constant symbols come from the signature of the theory $\Theta$. Examples are integral or real numbers, as well as enumerations of symbolic values such as Red or Blue for colors, or Silver or Gold for customer categories.

\section{Symbols for variables}

Variable symbols denote free or quantified variables. As we shall see, variables are used in rule programs to handle the objects matched and acted upon by the rules. Variable symbols come in infinite number and their set is noted Var.

Since variables hold objects, they are usually named $o$ or $o_{1}, o_{2}, \ldots$ In examples, where the objects handled through the variables are given a business meaning such as persons or shopping carts, they might be named with more meaningful letters such as $p$ or $c$. Conversely, dummy or quantified variables are usually named $x, y$, etc. Metavariables are noted $\xi$ or $\zeta$.

\section{Type names}

Type names are symbols that denote object types as defined by the BRMS-based application. The set of type names is finite and is noted Types.

A common generic name for a type is $T$, or $T_{1}, T_{2}, \ldots$ In examples we might use more business-oriented names such as Person, Car, etc.

\section{Attribute symbols}

Attribute symbols denote attributes or fields, that is, structural features of objects. An object attribute has a name and a value. The symbol denoting an 
attribute carries the attribute name. The infinite set of all attribute symbols is noted Attr.

Examples of attribute symbols are age or value. A common generic name for an attribute symbol is $f$ (for field, or feature).

\section{Operators}

Operators are function symbols used in infix notation, which are introduced by each value types.

All data types use equality, Booleans introduce logical connectors, and numbers introduce arithmetic and comparison operators.

Objects also use equality, and two additional operators. The dot is used for attribute reference: for example, p.age denotes the age attribute of the object held by the variable $p$. The colon is used to express an object typing expression: for example, $p$ : Person denotes that the variable $p$ is expected to hold objects of type Person.

\subsection{Expressions and Formulas}

Non-quantified formulas are Boolean expressions; quantified formulas are built out of them. All formulas are flat, meaning that chains of attribute references are not allowed: p.spouse.age $>20$ must be written $q=p$.spouse $\wedge$ q.age $>20$.

In addition, a type system establishes a link between variables, type names, and the attribute symbols referenced on the variables of a well-typed expression or formula.

\subsubsection{Parameterized Languages of Expressions and For- mulas}

Expressions and formulas are built with the symbols defined above. Depending on the context, we might want to restrict which variables are allowed to appear in an expression or a formula. To this end, we define parameterized languages of expressions and formulas, based on a set of variables $V \subseteq$ Var.

In Fig. 3.1, we give a definition of a few parameterized languages, namely the language of expressions $\mathcal{E}(V)$, the language of Boolean expressions or nonquantified formulas $\mathcal{B}(V)$, and the language of possibly quantified formulas $\mathcal{F}(V)$.

For these definitions we use grammars in Backus-Naur format. We slightly extend the notation for non-terminals, such as $\langle$ expression $\rangle$, to link a language with the root non-terminal of its grammar, as for example in $\langle$ expression : $\mathcal{E}(V)\rangle$. Also, we sometimes introduce shortcuts for a non-terminal, as $\varphi$ in $\langle$ formula : $\varphi \in \mathcal{F}(V)\rangle$.

In the grammar of Fig. 3.1, one can note the presence of the theory-dependent non-terminals $\langle\Theta$ expr. $\rangle$ and $\langle\Theta$ formula $\rangle$. With these extension points, we can integrate expressions and formulas from the application theory into the language. In Section 3.3.4, we give the definitions for the expressions and formulas that we use in examples. 


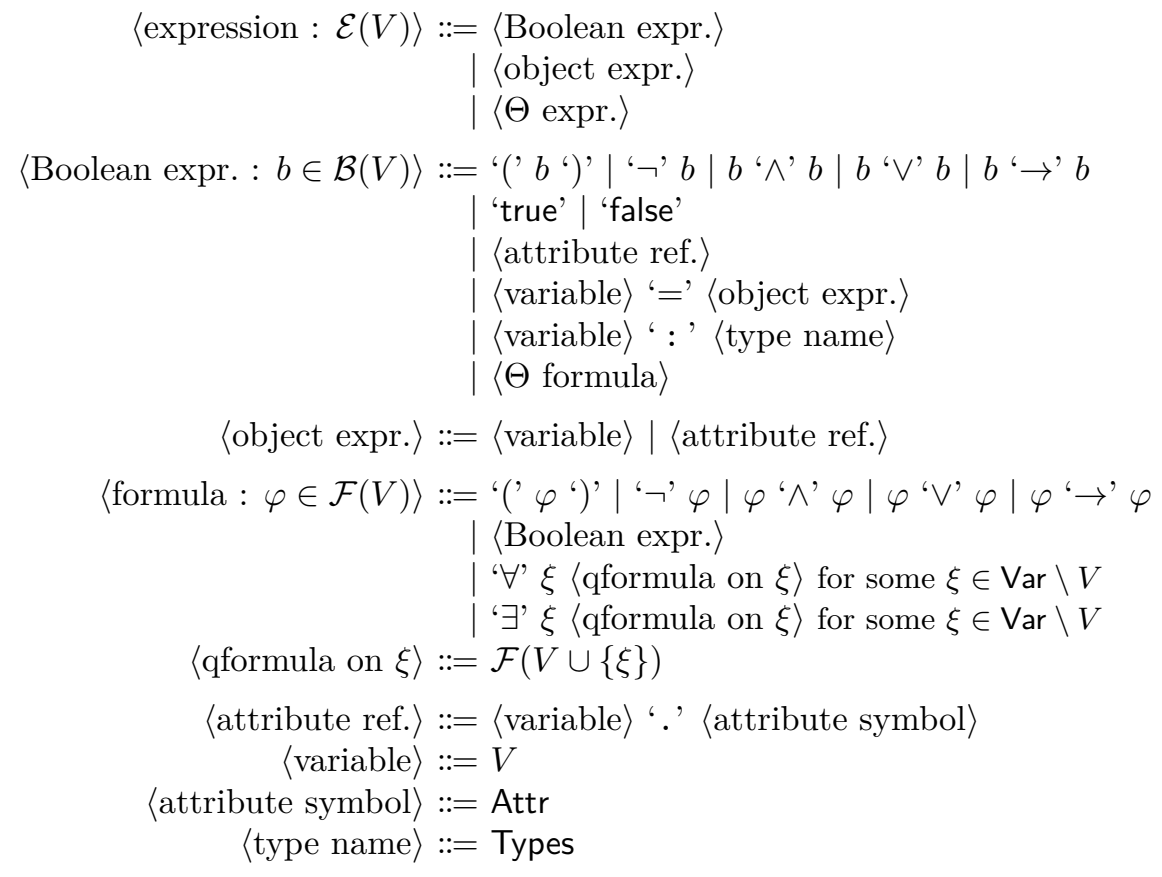

Figure 3.1: Expressions and formulas.

Also, the grammar does not enforce any typing constraint. For example, an attribute reference can occur as a Boolean expression or as an object expression. These cases are handled in the semantic interpretation in Chapter 4, by defining for example that Blue $=2$ is false. Similarly, constraints that result from object types are not enforced by the grammar, but will be enforced through the notion of well-typed expressions and formulas, introduced in Section 3.3.2.

\section{Variables and Attribute Symbols of Expressions and Formulas}

Let us consider a set of variables $V \subseteq \operatorname{Var}$ and a formula $\varphi \in \mathcal{F}(V)$. We note $\operatorname{var}(\varphi)$ the set of variables appearing in $\varphi$.

Variables in a formula appear either under the scope of a quantifier, in which case they are called bound variables, or not, in which case they are called free variables. The set of free variables in $\varphi$ is noted $\operatorname{free}(\varphi)$. Formulas with no free variables are called closed formulas. Given the definition of $\mathcal{F}(V)$, the set $V$ is not a superset of all the variables in a formula $\varphi \in \mathcal{F}(V)$, but only of the free variables in $\varphi$. If $\varphi \in \mathcal{B}(V)$, that is, it contains no quantifier, then free $(\varphi)=\operatorname{var}(\varphi)$ and $\operatorname{var}(\varphi) \subseteq V$. Conversely, $\mathcal{F}(\emptyset)$ is the set of all closed formulas.

For a formula $\varphi \in \mathcal{F}(V)$, we note $\operatorname{Attr}(\varphi)$ the set of attribute symbols appearing in $\varphi$. In addition, given a variable $\xi \in \operatorname{Var}$, we note $\operatorname{Ref}(\xi, \varphi)$ the set of attribute symbols appearing in $\varphi$ in an attribute reference where the variable is $\xi$. Finally, we note $\operatorname{Free}(\varphi)=\cup_{\xi \in \text { free }(\varphi)} \operatorname{Ref}(\xi, \varphi)$ the set of attribute symbols appearing in references to attributes of free variables in $\varphi$.

We make similar definitions for expressions: given an expression $e \in \mathcal{E}(V)$ and a variable $\xi \in \operatorname{Var}$, we define the sets $\operatorname{var}(e), \operatorname{Attr}(e)$, and $\operatorname{Ref}(\xi, e)$. Since 
expressions contain no quantifiers, there is no notion of bound or free variable in expressions.

Example. Given the formula

$$
\varphi \equiv \forall p(\text { p.age }>60 \wedge \text { p.ancestor }=q) \vee q . \text { age }=0
$$

we have

$$
\begin{aligned}
\operatorname{var}(\varphi) & =\{p, q\} & \operatorname{Attr}(\varphi) & =\{\text { age, ancestor }\} \\
\operatorname{free}(\varphi) & =\{q\} & \operatorname{Ref}(p, \varphi) & =\{\text { age, ancestor }\} \\
\operatorname{Free}(\varphi) & =\{\text { age }\} & \operatorname{Ref}(q, \varphi) & =\{\text { age }\} .
\end{aligned}
$$

\subsubsection{Well-Typed Expressions and Formulas}

We use a basic type framework inspired from [KLW95]. We introduce below only the concepts that we need.

In our framework, a type system contains type names and type definitions. Type names are symbols as exposed in Section 3.2, and denote what is called classes in the object-oriented paradigm. Type definitions provide a set of attribute symbols for each type, which corresponds in the object-oriented paradigm to the set of attributes defined in the class.

It results naturally that when a variable is equipped with a type, well-typed expressions and formulas should not contain references to other attributes of this variable than those included in the definition of its type.

Definition 3.1. A type system $\mathcal{T}=($ Types, $\alpha)$ consists of

(i) a set of type names noted Types;

(ii) a type definition function $\alpha$ : Types $\rightarrow \wp($ Attr) that maps each type name $T \in$ Types onto a finite set of attribute symbols $\alpha(T) \subset$ Attr.

To simplify the definitions and notations that will occur later, we require type definitions to be disjoint, that is, given any two distinct types names $T_{1}, T_{2} \in$ Types, we require that $\alpha\left(T_{1}\right) \cap \alpha\left(T_{2}\right)=\emptyset$.

This requirement for type definitions to be disjoint amounts to forbidding attribute symbol overloading. For example, we forbid the age of a person and the age of a car from being denoted by the same attribute symbol. Such a restriction does not reduce the expression power of type systems. In practice, it is usually hidden to the programmer through some mangling process performed by the language compiler. Also, we do not consider subtyping, as occurs in the object-oriented paradigm with subclasses, because it does not introduce significant differences in our formalism, and is not our focus.

We note $\operatorname{Attr}(\mathcal{T})=\bigcup_{T \in \text { Types }} \alpha(T)$ the set of all attribute symbols used in type definitions of our type system. This set is finite by construction.

We now introduce the definition of a type constraint on a set of variables, and we build on this concept to define well-typed expressions and formulas.

Definition 3.2. A type constraint on variables is a function $\tau: V \rightarrow$ Types, with $V \subseteq \operatorname{Var}$, that associates a type name with each variable in $V$. We note $\operatorname{Dom}(\tau)$ the domain $V$ of the type constraint. 
Definition 3.3. Given a set of variables $V \subseteq \operatorname{Var}$, a type system $\mathcal{T}=($ Types, $\alpha)$ and a type constraint $\tau$ :

(i) An expression $e \in \mathcal{E}(V)$ is well-typed with respect to $\mathcal{T}$ and $\tau$ if for each variable $\xi \in \operatorname{var}(e) \cap \operatorname{Dom}(\tau)$ one has $\operatorname{Ref}(\xi, e) \subseteq \alpha(\tau(\xi))$.

(ii) A formula $\varphi \in \mathcal{F}(V)$ is well-typed with respect to $\mathcal{T}$ and $\tau$ if for each variable $\xi \in \operatorname{var}(\varphi) \cap \operatorname{Dom}(\tau)$ one has $\operatorname{Ref}(\xi, \varphi) \subseteq \alpha(\tau(\xi))$.

Since we work with a fixed type system, we will usually omit citing it, and say that an expression or a formula is well-typed for a given type constraint.

\subsubsection{Formulas Are Flat}

Note that the grammar for $\mathcal{B}(V)$ imposes the constraint that an object can be referred to only either through a variable, or through the reference to an attribute of a variable. That is, the grammar excludes chains of attribute references, forbidding for example p.parent.age. The resulting formulas are called flat by analogy with flat terms where variables occur only at depth 0 or 1 .

As will become obvious when we introduce the semantics of formulas, this is not a proper restriction. In short, a formula from an extension of some $\mathcal{F}(V)$ language with chains of attribute references can be transposed into an equivalent formula of the strict $\mathcal{F}(V)$ language by introducing existentially-quantified fresh variables. For non-quantified formulas, the transposition from an extension of some $\mathcal{B}(V)$ language with chains of attribute references includes the introduction of additional variables, resulting in formulas from some $\mathcal{B}\left(V^{\prime}\right)$ where $V^{\prime}$ is a strict superset of $V$.

For example, the formula p.parent.age $>60$ will be written $q=$ p.parent $\wedge$ q.age $>60$.

\subsubsection{Theory Used in Examples}

The definition of the languages of expressions and formulas above opens the door for theory-specific expressions and formulas. In the subsequent examples we use this mechanism to include numbers and symbolic values in the rules.

The grammar in Fig. 3.2 gives the derivations to complete the grammar of Fig. 3.1 with numbers and symbolic values, thus defining the languages used in our examples.

$$
\begin{aligned}
& \langle\Theta \text { expr. }\rangle::=\langle\text { num. expr. }\rangle \mid\langle\text { symb. expr. }\rangle
\end{aligned}
$$

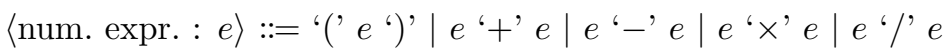

$$
\begin{aligned}
& \text { | NUMBER } \\
& \text { | 〈attribute ref. }\rangle \\
& \langle\text { symb. expr. }::=\text { SYMBOLIC-VALUE }|\langle\text { attribute ref. }\rangle \\
& \langle\Theta \text { formula }\rangle::=e^{\prime}={ }^{\prime} e\left|e^{\prime}<{ }^{\prime} e\right| e^{\prime} \leq ' e\left|e^{\prime}>{ }^{\prime} e\right| e^{\prime} \geq ' e \\
& \mid\langle\text { symb. expr. }\rangle \text { ' }=\text { ' }\langle\text { symb. expr. }\rangle
\end{aligned}
$$

Figure 3.2: Expressions and formulas on numbers and symbolic values (extension to Fig. 3.1). 


\subsection{Assignment}

The assignment of an expression to an attribute of an object held by a variable, is the statement that will constitute the actions of rules.

Our rule language contains only one statement: assignment of a value to an attribute of an object. Because the rule engine takes charge of the control, no control flow statement, such as conditionals or loops, is included in the action language for our rules.

However, we will later resort, in our reasoning about rule programs, to traditional sequential statements and to some statements found in parallel and nondeterministic programs. We will formally introduce these statements in Chapter 6 . For now we simply define the syntax of assignment, and its semantics in the next chapter.

The definition of assignment is based on the definition of expressions provided in Fig. 3.1. As a consequence, this definition is also parameterized by a set of variables $V \subseteq$ Var. In Fig. 3.3, we define the language of assignment $\mathcal{A}(V)$, which constitutes also the language for rule actions.

$$
\begin{aligned}
\langle\text { assignment }: \mathcal{A}(V)\rangle::=\langle\text { attribute ref. }\rangle ':='\langle\text { expression }: \mathcal{E}(V)\rangle \\
\langle\text { attribute ref. }\rangle::=\langle\text { variable }: V\rangle^{\prime} \cdot{ }^{\prime}\langle\text { attribute symbol : Attr }\rangle
\end{aligned}
$$

Figure 3.3: Assignment.

An assignment operates on an attribute reference, and assigns it an expression from the language $\mathcal{E}(V)$ as defined in Fig. 3.1. In particular, the expression can contain theory-specific terms.

Example. The following first assignment sets the value of the category attribute of the object held by variable $p$, to the constant Gold. The second one increments by 10 the value of the discount attribute of the object held by variable $c$.

(i) p.category $:=$ Gold

(ii) c.discount $:=$ c.discount +10

Definition 3.4. Let $x . f:=e$ be an assignment. Given a type system $\mathcal{T}=$ (Types, $\alpha$ ) and a type constraint $\tau$, the assignment is well-typed for $\tau$ if:

(i) the attribute on the left-hand side of the assignment is in the type definition of the variable, that is, $f \in \alpha(\tau(x))$; and

(ii) the expression $e$ is well-typed for $\tau$.

\subsection{Rules}

Rules are the building blocks of rule programs. Each rule handles a tuple of objects through a collection of variables. The rule guard is a formula in these variables, the rule action is an assignment to an attribute of one of them.

Definition 3.5. A rule $r=(\vec{o}, \vec{T}, g, a)$ consists of: 
(i) $\vec{o}=\left(o_{1}, \ldots, o_{m}\right):$ a tuple of variables;

(ii) $\vec{T}=\left(T_{1}, \ldots, T_{m}\right)$ : a tuple of type names of the same length, and called the types of the variables; together with the tuple of variables, the tuple of type names forms a type constraint $(\vec{o}: \vec{T})$ as defined in Definition 3.6 just below;

(iii) $g \in \mathcal{B}\left(\left\{o_{1}, \ldots, o_{m}\right\}\right):$ a non-quantified formula in the above variables, well-typed for the rule type constraint $(\vec{o}: \vec{T})$, and called the guard of the rule;

(iv) $a \in \mathcal{A}\left(\left\{o_{1}, \ldots, o_{m}\right\}\right):$ an assignment to an attribute of one of the above variables, well-typed for the rule type constraint $(\vec{o}: \vec{T})$, and called the action of the rule.

Definition 3.6. Given a rule $r=(\vec{o}, \vec{T}, g, a)$ involving a tuple of variables $\vec{o}=\left(o_{1}, \ldots, o_{m}\right)$ and a tuple of type names $\vec{T}=\left(T_{1}, \ldots, T_{m}\right)$, the rule type constraint for $r$ is the type constraint $\tau$, also noted $(\vec{o}: \vec{T})$, defined by

$$
\operatorname{Dom}(\tau)=\left\{o_{1}, \ldots, o_{m}\right\} \quad \text { and } \quad \tau\left(o_{j}\right)=T_{j} \quad \text { for } j=1, \ldots, m .
$$

Example. The following rule is made of two rule variables $c$ and $p$, respectively of type Cart and Person, a guard p.age $>60 \wedge$ c.value $>1000$, and an action c.discount $:=10$.

$$
r(c: \text { Cart, } p: \text { Person }): \text { p.age }>60 \& \text { c.value }>1000 \rightarrow \text { c.discount }:=10
$$

All rules in the examples will be expressed in this self-explanatory concrete syntax that provides the rule name, its variables and their types in parenthesis, and its guard and action separated by an arrow ${ }^{2}$.

\subsubsection{Rule Variables}

The variables of a rule $r=(\vec{o}, \vec{T}, g, a)$ are those in the tuple $\vec{o}=\left(o_{1}, \ldots, o_{m}\right)$. Their number $m \geq 0$ can vary from rule to rule, and is called the arity of the rule, also noted $|r|$.

The rule variables denote the objects that will be matched by the rule (as defined in Chapter 5). We refer to their set as $\operatorname{var}(r)=\left\{o_{1}, \ldots, o_{m}\right\}$. They appear as variables of the guard $g$ and/or in the assignment performed in the action $a$. To avoid spurious matches we require that $\operatorname{var}(r)=\operatorname{var}(g) \cup \operatorname{var}(a)$, that is, the tuple of rule variables contains no extra variable.

The rule variables are in essence dummy variables, in the sense that their actual names do not matter within the scope of one individual rule. As for the formal parameters in a procedure definition, what matters is their number and their order. In the previous example, the rule variables are named $c$ and $p$, in reference to shopping carts and persons, but could have any name.

Given a tuple $\vec{x}=\left(x_{1}, \ldots, x_{m}\right)$ of $m$ variables, we will note $r(\vec{x})$ the rule $r$ where the variables $o_{1}, \ldots, o_{m}$ have been replaced by the variables $x_{1}, \ldots, x_{m}$, respectively.

\footnotetext{
${ }^{2}$ In this thesis, an arrow is used in three situations: for logical implication, in the concrete syntax of rules introduced here, and in guarded commands introduced in Chapter 7. Although the context should make the meaning of arrows clear, we will use the regular arrow $\rightarrow$ for logical implication, and special arrows for the other two cases, namely $\rightarrow$ in rules and $\rightarrow$ in guarded commands.
} 
When several rules are considered together, it is important to be conscious that the variables of each rule are distinct. Although it is a common practice, that we will often adopt in examples, to reuse the variable names from rule to rule, an unambiguous description of a set of rules will introduce distinct variable names for each rule. For instance, the variables of two rules $r_{1}=\left(\vec{o}_{1}, \vec{T}_{1}, g_{1}, a_{1}\right)$ and $r_{2}=\left(\vec{o}_{2}, \vec{T}_{2}, g_{2}, a_{2}\right)$ will be noted $\vec{o}_{1}=\left(o_{1}^{1}, \ldots, o_{m_{1}}^{1}\right)$ and $\vec{o}_{2}=\left(o_{1}^{2}, \ldots, o_{m_{2}}^{2}\right)$.

The type names $T_{1}, \ldots, T_{m}$ provide a type constraint on the variables, that will have to be complied with by the rule guard and action. The above comment on variable names being distinct between rules applies to type names, with the type names of some rule $r_{i}$ having to be noted $\vec{T}_{i}=\left(T_{1}^{i}, \ldots, T_{m_{i}}^{i}\right)$. However, in a rule program the same type name can, and usually will, occur several times as the type of variables of several rules. It is the case in the rule program given as an example in Section 3.6.

\subsubsection{Rule Guard}

The guard of a rule $r=(\vec{o}, \vec{T}, g, a)$ with $\vec{o}=\left(o_{1}, \ldots, o_{m}\right)$ is a non-quantified formula $g \in \mathcal{B}\left(\left\{o_{1}, \ldots, o_{m}\right\}\right)$. Its variables are all among the rule variables.

The guard is well-typed with respect to the rule type constraint $(\vec{o}: \vec{T})$, meaning that it contains references to no other attribute of each variable $o_{j}$ than those included in the definition of the type $T_{j}$, for $j=1, \ldots, m$. In the previous example, this implies that value $\in \alpha$ (Cart) and age $\in \alpha$ (Person).

As noted in Section 3.3.3, the guard of a rule is a flat formula. This means that no chains of attribute reference are allowed, and represents no loss in expression power, at the possible cost of introducing new rule variables.

As a result, all the objects involved in the guard of a rule are directly held by one of the rule variables. This will make it easier to control interference between rules.

\subsubsection{Rule Action}

The action of a rule $r=(\vec{o}, \vec{T}, g, a)$ with $\vec{o}=\left(o_{1}, \ldots, o_{m}\right)$ is a single assignment, of the form $o_{1} \cdot f_{r}:=e_{r}$, where $f_{r}$ is some attribute symbol and $e_{r}$ an expression that involves the rule variables, their attributes, constants, etc. To simplify notations and without loss of generality, we fix that the object whose attribute is set in a rule action, is the one held by the first rule variable $o_{1}$.

The action is also well-typed with respect to the rule type constraint $(\vec{o}: \vec{T})$. In the previous example, this additionally implies that discount $\in \alpha$ (Cart).

Contrary to the constraint posed on rule guards to be flat formulas, the limitation of rule actions to only one assignment affects the expression power of rule programs. For example, a rule that implements a reset of a customer score when upgrading its category could be expressed as follows:

$$
r(\mathrm{p}: \text { Person }): \text { p.cat }=\text { Gold \& p.score }>1000 \rightarrow \text { p.cat }:=\text { Plat } ; \text { p.score }:=0
$$

Yet this rule cannot be rephrased with one or several rules whose action is limited to a single assignment.

The motivation for this restriction on the actions of rules is simplification. The work on correctness and verification exposed in Parts II and III is largely independent from this aspect and could be reformulated for more complex rule actions, at the cost of more complicated formulas [BDS12]. 


\subsection{Rule Programs}

A rule program is a set of rules. The fact that rules are not ordered within a rule program underlines the declarative nature of rule programming.

Definition 3.7. A rule program is a finite set of rules $\mathcal{R}=\left\{r_{1}, \ldots, r_{n}\right\}$ with $n \geq 0$. Each rule $r_{i}$ (for $\left.i=1, \ldots, n\right)$ has

(i) its own variables $\vec{o}_{i}=\left(o_{1}^{i}, \ldots, o_{m_{i}}^{i}\right)$ and their types $\vec{T}_{i}=\left(T_{1}^{i}, \ldots, T_{m_{i}}^{i}\right)$, which define the rule type constraint $\left(\vec{o}_{i}: \vec{T}_{i}\right)$;

(ii) its own guard $g_{i} \in \mathcal{B}\left(\left\{o_{1}^{i}, \ldots, o_{m_{i}}^{i}\right\}\right)$ well-typed for $\left(\vec{o}_{i}: \vec{T}_{i}\right)$; and

(iii) its own action $a_{i} \in \mathcal{A}\left(\left\{o_{1}^{i}, \ldots, o_{m_{i}}^{i}\right\}\right)$ well-typed for $\left(\vec{o}_{i}: \vec{T}_{i}\right)$, with $a_{i} \equiv$ $o_{1}^{i} \cdot f_{r_{i}}:=e_{r_{i}}$.

Example. Consider the rule program $\mathcal{R}=\left\{r_{1}, r_{2}, r_{3}\right\}$ composed of the following rules:

$$
\begin{array}{r}
r_{1}(c: \text { Cart, } p: \text { Person }): \text { p.age }>60 \& \text { c.value }>1000 \rightarrow \text { c.discount }:=10 \\
r_{2}(p: \text { Person }): \text { p.cat }=\text { Gold } \rightarrow \text { p.bonus }:=20 \\
r_{3}(c: \text { Cart, } p: \text { Person }): \text { p.bonus }>15 \& \text { c.value }>2000 \rightarrow \text { c.discount }:=30
\end{array}
$$

Here we followed the tradition, and reused the variable names across the rules. However, when manipulating these rules together, we would distinguish the variables, and for example describe the rules as follows:

- Rule $r_{1}$ is made of the variables $\vec{o}_{1}=\left(c_{1}, p_{1}\right)$, the type names $\vec{T}_{1}=$ (Cart,Person), the guard $g_{1} \equiv p_{1}$.age $>60 \wedge c_{1}$.value $>1000$, and the action $a_{1} \equiv c_{1}$.discount $:=10$.

- Rule $r_{2}$ is made of the variables $\vec{o}_{2}=\left(p_{2}\right)$, the type names $\vec{T}_{2}=$ (Person), the guard $g_{2} \equiv p_{2} . c a t=$ Gold, and the action $a_{2} \equiv p_{2}$.bonus $:=20$.

- Rule $r_{3}$ is made of the variables $\vec{o}_{3}=\left(c_{3}, p_{3}\right)$, the type names $\vec{T}_{3}=$ (Cart,Person), the guard $g_{3} \equiv p_{3}$.bonus $>15 \wedge c_{3}$.value $>2000$, and the action $a_{3} \equiv c_{3}$.discount $:=30$.

Given a rule program $\mathcal{R}$, we note $\operatorname{var}(\mathcal{R})$ the set gathering all the variables of all the rules of $\mathcal{R}$. That is, if $\mathcal{R}=\left\{r_{1}, \ldots, r_{n}\right\}$ with $r_{i}=\left(\vec{o}_{i}, \vec{T}_{i}, g_{i}, a_{i}\right)$ for each $i=1, \ldots, n$, we have

$$
\operatorname{var}(\mathcal{R}) \stackrel{\text { def }}{=} \bigcup_{i=1}^{n}\left\{o_{1}^{i}, \ldots, o_{\left|r_{i}\right|}^{i}\right\} .
$$

In the example above, we have $\operatorname{var}(\mathcal{R})=\left\{c_{1}, c_{3}, p_{1}, p_{2}, p_{3}\right\}$. 


\section{Chapter 4}

\section{States and State Assertions}

The syntactic elements exposed in the previous chapter allow us to build rule programs, as well as assertions for reasoning about them. In the present chapter, we use first-order logic structures and variable valuations to give a meaning to these syntactic elements.

\section{Example}

For the purpose of giving expressions a value, and formulas a truth value, firstorder logic structures provide a semantic interpretation of constant symbols, attribute symbols, and operators; and variable valuations provide an object value for each variable symbol.

We shall see that constant symbols and operators are interpreted in a classical way, and that attribute symbols are interpreted by functions providing for each object the value of the attribute. With this informal description of our semantics, let us consider an example. (This example is discussed in a more complete description in Section 4.1.5.)

In all examples, including this one, we will use names such as Alice, Bob, Alfa, or Beetle to denote objects; that is, concrete objects that are the values of variables.

A valuation $\nu$ assigning the object Alice to the variable $q$ and the object Beetle to the variable $c$ is noted

$$
\nu: q \mapsto \text { Alice } \quad c \mapsto \text { Beetle. }
$$

An example of a first-order logic structure consists in the functions that interpret each attribute symbol. Each such function maps the objects (Alice and the others) onto values. Let us assume that the attribute symbols defined by our type system are age, car, and color. The first-order logic structure $s$ given below defines the corresponding three functions, where age $e^{s}$ denotes the function that interprets the attribute symbol age, etc.:

$$
\begin{aligned}
& a g e^{s}: \text { Alice } \mapsto 21 \mathrm{car}^{s} \text { : Alice } \mapsto \text { Beetle } \operatorname{color}^{s}: \text { Alfa } \mapsto \text { Red } \\
& \text { Bob } \mapsto 80 \quad \text { Bob } \mapsto \text { Alfa } \quad \text { Beetle } \mapsto \text { Yellow. } \\
& \text { Carol } \mapsto 75 \quad \text { Carol } \mapsto \text { Alfa }
\end{aligned}
$$


The first-order logic structure $s$ and the variable valuation $\nu$ above make the formulas below valid:

$$
\begin{aligned}
& \varphi \equiv \text { c.color }=\text { Yellow } \\
& \psi \equiv \forall p \forall w((\text { age }>60 \wedge w=p . \text { car }) \rightarrow w . \text { color }=\text { Red }) \wedge \text { q.age }=21 .
\end{aligned}
$$

This can be explained, still informally, as follows. The formula $\varphi$ holds because the value onto which $\nu$ maps $c$ is Beetle, and the image by $\operatorname{color}^{s}$ of Beetle is Yellow. In the formula $\psi$ the first, quantified conjunct is true because in $s$, all persons above 60 own the car Alfa, the color of which is red; the second conjunct in $\psi$ is true because the valuation assigns the object Alice to the variable $q$, and the age of Alice in $s$ is 21 .

\subsection{States Are First-Order Logic Structures}

The expressions and formulas defined in Section 3.3 are interpreted by firstorder logic structures, which serve as states for reasoning about the execution of rule programs. This section defines the domain and interpretation function of states.

A state is a first-order logic structure. Its main contribution is to interpret each attribute symbol by a function that maps objects onto values. Variables are mapped onto objects by valuations, also introduced in this section.

An expression or a formula is thus given a value by both a state and a valuation.

A state is a first-order logic structure. It provides a domain and an interpretation function for constant symbols, attribute symbols, and operators. Throughout this section, we define the domain and interpretation function of states, leading to the formal definition of a state in Section 4.1.5.

A state and a valuation (defined in Section 4.1.2) are enough to give expressions a value, and formulas a truth value. In Section 4.4, we introduce another construction on first-order logic structures to describe, together with a valuation, the effect of a statement.

\subsubsection{Domain}

The common domain for all the first-order logic structures we will use, is $\mathcal{D}=$ $\mathbb{D} \cup \mathbb{V}$, where $\mathbb{D}$ is the object space and $\mathbb{V}$ is the value space, as defined below.

We note $\mathbb{D}$ the infinite set of all objects. An object has a unique identity. The identities of two objects can be compared for equality; no other operations are available on the identities of objects.

We note $\mathbb{V}$ the set of all (non-object) values considered, from Booleans to application-specific values described by the fixed theory $\Theta$ which acts as a parameter to our rule language. In this chapter we give the interpretation of the parameterized language, plus an interpretation of the theory used in examples, including numbers and symbolic values.

\subsubsection{Variable Valuations}

Definition 4.1. A valuation is a function $\nu: \operatorname{Var} \rightarrow \mathbb{D}$ assigning an object to each variable symbol. 
The role of a valuation in the interpretation of expressions and formulas is to provide object values for variables.

As already mentioned when defining variable symbols in Section 3.2, and as formalized by the definition above, variables hold objects. Our rule language does not include scalar, non-object variables. Allowing scalar variables as rule variables (see Section 3.5.1) would diverge from the reality of rule programs as handled by Business Rules Management Systems, where rules only match objects from the working memory (defined in the next chapter). Adding scalar variables to expressions and formulas while forbidding them as rule variables is possible, but would add little value to our rule language in terms of expressive power.

A common formalization of production rule programs uses the notion of substitution to model a rule matching facts (see for example [FL92, CKMM04, dSMHP10]). In these non object-oriented settings, facts are ground terms and a rule contains patterns, which are non ground terms; a rule is said to match a fact if there exists a substitution that maps the patterns of the rule onto the fact (for positive patterns). Our setting is not fundamentally different: as we shall see below, a state interprets the attribute symbols that occur in the rule guard by functions; the valuations just defined are used to "ground" these functions on objects.

\subsubsection{Interpretation of Expressions}

\section{Constants}

The constant symbols for Booleans are interpreted by the corresponding Boolean values. When needed, we note $\mathbb{B}=\{$ true, false $\}$ the set of Boolean values. Operations on Booleans and on Boolean expressions are exposed in detail in Section 4.1.4 below.

Numbers are interpreted by integral ones (from $\mathbb{Z}$ ) or real ones (from $\mathbb{R}$ ) or complex ones, etc. - as specified by the fixed theory $\Theta$. This choice has an impact only when decidability or completeness comes into play; we will then discuss it.

The constant symbols for symbolic values (such as Red or Child) are interpreted by values with no other features than their identities. All symbolic values are distinct, which is usually referred to as the Unique Name Assumption [RN09]. them.

Also, symbolic values are not objects, and as such cannot be compared to

In some cases, such as completeness analysis of rule programs (see Section A.5.2), symbolic values are grouped into enumerations, such as colors, genders, customer categories, etc. Each enumeration is finite, which is usually referred to as the Domain Closure Assumption (on each enumeration) [RN09].

\section{Attributes}

An attribute symbol $f \in$ Attr is interpreted in a state $s$ by a function noted $\mathcal{I}_{s}(f)$ or $f^{s}$. This function is total, and defined from $\mathbb{D}$ to $\mathcal{D} \cup\{\perp\}$. The $\perp$ value is used to define the function on objects that have no value for the attribute. 
Definition 4.2. Given a state $s$ and an attribute symbol $f$, the domain of $f$ in $s$ is the set of objects for which the function $f^{s}$, which interprets the attribute symbol in the state, has a non- $\perp$ value. We note

$$
\operatorname{Dom}_{s}(f)=\left\{O \in \mathbb{O} \mid f^{s}(O) \neq \perp\right\} .
$$

We impose as an additional constraint on our states that each attribute symbol must have a finite domain, that is, the set of objects with a non- $\perp$ value in a given state must be finite for all attributes.

\section{Attribute references}

Given this interpretation of attribute symbols, the reference to an attribute of an object is simply interpreted by the application of the corresponding function.

For example, in a state where the attribute symbol age is interpreted by a function $a g e^{s}$ such that $a g e^{s}$ (Alice) $=10$, and under a valuation that maps $p$ onto Alice, the attribute reference p.age is given the value 10 .

\section{Numeric expressions}

Faced with the question of whether to interpret numbers in the mathematical sense, or to treat them as machine numbers, with such behaviors as modularity or rounding errors, we decide to follow the mathematical interpretation of operations on numbers.

This decision is motivated by the focus of Business Rules Management Systems toward domain experts rather than programmers, and the belief that the business rules authors have this semantics in mind rather than the machine one. With this choice, we favor finding errors in reasoning, such as $x^{2}<0$, which is not always false with machine numbers, over tracking behaviors related to the specifics of machine implementations of numbers.

We augment the classical computation rules of number arithmetics with one rule due to the introduction of the $\perp$ value. When one of the operands in an arithmetic operation is $\perp$, then the result of the operation is $\perp$. Similarly when one of the operands in an arithmetic operation is not a number. For example, $\perp+3$ and $5-$ Blue both equal $\perp$.

\subsubsection{Interpretation of Formulas}

\section{Truth value of a formula}

We say that a formula $\varphi \in \mathcal{F}(V)$ holds in a given state $s$ under a valuation $\nu$ when the interpretation function of the state defined in this section, together with the variable-to-object mapping defined by the valuation, gives true as the truth value of the formula. We note this $s, \nu \models \varphi$.

For example, under a valuation $\nu$ such that $\nu(p)=$ Alice, and in a state $s$ where the attribute symbol age is interpreted by a function age $e^{s}$ such that $\operatorname{age}^{s}($ Alice $)=10$, one has: $s, \nu \models$ p.age $<21$.

The rest of this section defines the interpretation of formulas. This interpretation provides a truth value for the formulas, by induction on their structure.

In addition, by $\varphi \Rightarrow \psi$ we mean that for any state $s$ and valuation $\nu$, if $s, \nu \models \varphi$ then $s, \nu \models \psi$. And by $\varphi \Longleftrightarrow \psi$ we mean that for any state $s$ and valuation $\nu, s, \nu \models \varphi$ if and only if $s, \nu \models \psi$. 


\section{Typing expression}

Informally, the typing expression $o: T$ expresses that the object held by $o$ complies with the definition given to the object type $T$ by the type definition function $\alpha$ of the fixed type system. The definition of a type is a set of attribute symbols; an object complies with such a definition when exactly the attributes denoted by the attribute symbols in the definition have a non- $\perp$ value on the object.

Formally, let $o$ be a variable and $T$ be a type name. The Boolean expression $o: T$ holds in a state $s$ under a valuation $\nu$ if

for all $f \in \alpha(T)$ and $\bar{f} \in \operatorname{Attr} \backslash \alpha(T)$ one has $f^{s}(\nu(o)) \neq \perp$ and $\bar{f}^{s}(\nu(o))=\perp$.

\section{Equalities}

Object or symbolic value comparison is performed on their respective identities. Numeric equality is defined in the classical way. An equality mixing objects, symbolic values or numbers (as in Blue $=2$ ) is false.

In all three cases, if $\perp$ is involved in an equality, the equality is false if one side, but not both, of the equality is $\perp$. In other words,

$$
\begin{array}{lll} 
& \perp=\perp & \text { is true } \\
\text { for all } x \in \mathcal{D} & x=\perp & \text { is false. }
\end{array}
$$

\section{Inequalities between numbers}

Inequalities (built with the comparison operators $<,>, \leq, \geq$ ) between numbers are defined in the classical way. An inequality between a number and $\perp$ is always false. An inequality between $\perp$ and itself is true only if one of the non-strict comparison operators $\leq$ or $\geq$.

With these computation rules in the presence of $\perp$, the usual correspondences between the comparison operators, such as $x<y \Longleftrightarrow y>x$ or $x \leq y \Longleftrightarrow$ $x=y \vee x<y$ hold, with the exception of the correspondences for negated comparisons (such as $\neg(x<y) \Longleftrightarrow x \geq y$ ). These must be replaced with the following ones:

$$
\begin{aligned}
& \neg(x<y) \Longleftrightarrow x=\perp \vee y=\perp \vee x \geq y \\
& \neg(x>y) \Longleftrightarrow x=\perp \vee y=\perp \vee x \leq y \\
& \neg(x \leq y) \Longleftrightarrow x \neq y \wedge(x=\perp \vee y=\perp \vee x \geq y) \\
& \neg(x \geq y) \Longleftrightarrow x \neq y \wedge(x=\perp \vee y=\perp \vee x \leq y) .
\end{aligned}
$$

\section{Logical connectors}

The truth value of formulas built with the logical connectors $\neg, \wedge, \vee$, and $\rightarrow$ is defined in the classical way, with $\perp$ being treated as false. The computation 
rules for logical connectors in the presence of $\perp$ are thus:

$$
\begin{array}{rll} 
& \perp & \text { is false } \\
& \neg \perp & \text { is true } \\
\text { for all } b \in \mathbb{B} \cup\{\perp\} & \perp \wedge b & \text { is false } \\
\text { for all } b \in \mathbb{B} \cup\{\perp\} & \perp \vee b & \text { has the truth value of } b \\
\text { for all } b \in \mathbb{B} \cup\{\perp\} & \perp \rightarrow b & \text { is true } \\
\text { for all } b \in \mathbb{B} & b \rightarrow \perp & \text { is false. }
\end{array}
$$

Note that the only potential occurrence of $\perp$ as an operand of a logical connector comes from a reference to an object attribute with a Boolean value.

\section{Quantifiers}

Let us consider some set of variables $V \subseteq \operatorname{Var}$, as well as a formula $\varphi \in \mathcal{F}(V)$ and a variable $\xi \in V$.

The formula $\forall \xi \varphi$ holds in a state $s$ under a valuation $\nu$, if and only if $\varphi$ holds in $s$ under all valuations that coincide with $\nu$ on all other variables of $V$ than $\xi$. That is,

$$
s, \nu \models \forall \xi \varphi \quad \text { iff } \quad s, \nu^{\prime} \models \varphi \quad \text { for any } \nu^{\prime} \text { s.t. } \nu^{\prime}(\zeta)=\nu(\zeta) \text { for } \zeta \in V \backslash\{\xi\} .
$$

The formula $\exists \xi \varphi$ holds in a state $s$ under a valuation $\nu$, if and only if $\varphi$ holds in $s$ under at least one valuation that coincides with $\nu$ on all other variables of $V$ than $\xi$. That is,

$$
s, \nu \models \exists \xi \varphi \quad \text { iff } \quad s, \nu^{\prime} \models \varphi \quad \text { for some } \nu^{\prime} \text { s.t. } \nu^{\prime}(\zeta)=\nu(\zeta) \text { for } \zeta \in V \backslash\{\xi\} \text {. }
$$

\section{Typed quantification}

Consider the quantified formula $\forall p$ (p.age $>21$ ). Per the definition of quantifiers above, this formula holds in a state $s$ and under a valuation $\nu$ if and only if $s, \nu^{\prime} \models$ p.age $>21$ for any valuation $\nu^{\prime}$, that is, if age $(O)>21$ for any object $O \in \mathbb{D}$. It can happen that the given state $s$ interprets the attribute symbol age as a function assigning a value greater than 21 to all objects on which it has a non- $\perp$ value. However, the interpretation of attribute symbols (in Section 4.1.3) states that these objects, known as the domain of age in $s$, are in finite number. On all the other objects, we have $a g e^{s}(O)=\perp$. As a result, $\forall p(p$.age $>21)$ never holds.

What is missing here for this simple quantified formula to avoid being trivially invalid, is the information that only a subset of the objects is to be considered, namely a subset on which the attribute age is guaranteed to have a non- $\perp$ value. Providing this information is the intent of object types. Assume that the Person type has the age attribute in its definition, the formula $\forall p(p$ :Person $\rightarrow p$.age $>21)$ expresses what was originally intended.

Such a case is so common that we define an abbreviation for it. Given a formula $\varphi \in \mathcal{F}(V)$, a variable $\xi \in V$, and a type name $T \in$ Types:

(i) we note $\forall \xi: T \varphi$ as an abbreviation for $\forall \xi(\xi: T \rightarrow \varphi)$;

(ii) we note $\exists \xi: T \varphi$ as an abbreviation for $\exists \xi(\xi: T \wedge \varphi)$. 


\subsubsection{States}

Definition 4.3. A state is a first-order logic structure $s=\left(\mathcal{D}, \mathcal{I}_{s}\right)$ over the signature $\Sigma$, whose domain $\mathcal{D}$ is defined in Section 4.1.1, and whose interpretation function $\mathcal{I}_{s}$ conforms with the definitions of Sections 4.1.3 and 4.1.4.

Example. Let us consider again the introductory example, this time in its full description:

$$
\begin{aligned}
\text { Types }= & \{\text { Person }, \text { Car }\} \\
\alpha(\text { Person })= & \{\text { age }, \text { car }\} \\
\alpha(\text { Car })= & \{\text { color }\} \\
\operatorname{Var}= & \{p, q, w, x, \ldots\} \\
\mathbb{D}= & \{\text { Alice }, \text { Bob }, \text { Carol }, \text { Alfa, Beetle }, \ldots\} \\
\mathbb{V}= & \{\ldots,-2,-1,0,1,2, \ldots, \text { Red }, \text { Yellow }, \ldots\} \\
\varphi \equiv & \forall p: \text { Person } \forall w: \text { Car }((\text { p.age }>60 \wedge w=p . \text { car }) \rightarrow \text { w.color }=\text { Red }) \\
& \wedge \text { q.age }=21 .
\end{aligned}
$$

The formula $\varphi$ has only $q$ as free variable. The following state and valuation make it valid:

$$
\begin{array}{rlrlrl}
s: \text { age }^{s}: \text { Alice } & \mapsto 21 & \text { car }^{s}: \text { Alice } & \mapsto \text { Beetle } & \text { color }^{s}: \text { Alfa } & \mapsto \text { Red } \\
\text { Bob } & \mapsto 80 & \text { Bob } & \mapsto \text { Alfa } & \text { Beetle } & \mapsto \text { Yellow } \\
\text { Carol } & \mapsto 75 & \text { Carol } & \mapsto \text { Alfa } & \text { otherwise } & \mapsto \perp \\
\text { otherwise } & \mapsto \perp & \text { otherwise } & \mapsto \perp \\
\nu: \quad q & \mapsto \text { Alice } & & & \\
\text { otherwise } & \mapsto \text { anything. } & & & &
\end{array}
$$

From the definition of $s$ above follows the composition of the sets of objects of each type, which are used for interpreting the quantified sub-formulas:

$$
\begin{aligned}
\text { objects of type Person: } & \{\text { Alice, Bob, Carol }\} \\
\text { objects of type Car: } & \{\text { Alfa, Beetle } .
\end{aligned}
$$

The formula $\varphi$ would not be made valid if, for example, one of the following variations was made from the $s$ and $\nu$ defined above:

- Setting $\nu(q)=$ Bob would cause $q$.age $=21$ to have the truth value of $80=21$, that is, false.

- Setting $a g e^{s}$ (Alice) $=$ Red would cause $q$. age $=21$ to have the truth value of Red $=21$, that is, false.

- Setting $\operatorname{color}^{s}($ Alfa $)=$ Yellow would cause $w$.color $=$ Red to be false, for example for $p=$ Bob.

\subsection{State Assertions}

A formula expresses an assertion about the objects represented by its variables. A closed formula expresses a global assertion involving all objects, 
while a formula with free variables relates to a tuple of objects, such as those handled by a rule.

An assertion is given a truth value by a state and, when not closed, a variable valuation.

\subsubsection{Assertions}

State assertions, or in short assertions, are formulas from $\mathcal{F}($ Var $)$ as defined in Section 3.3, that is, first-order, flat, possibly quantified formulas.

As such, they are given a truth value by a state and a valuation, as defined in Section 4.1.4. The state is used to interpret the attribute symbols in the formula, and the valuation to assign an object to each variable. Note however that, in the valuation, only the values given to the free variables of the formula matter. This will be leveraged later, and is formalized in Lemma 4.1 below.

Lemma 4.1. If an assertion $\varphi \in \mathcal{F}(\operatorname{Var})$ holds in a state $s$ under a valuation $\nu$, then it holds in $s$ under any valuation $\nu^{\prime}$ that coincides with $\nu$ on $\operatorname{free}(\varphi)$.

Another practical fact is that the truth value of an assertion does not change when a free variable of the assertion is renamed. This property is formalized in Lemma 4.2 , where the notation $\varphi[\zeta / \xi]$ has the standard meaning of "the formula $\varphi$ in which $\zeta$ is substituted for all occurrences of $\xi "$.

Lemma 4.2. Let $\varphi \in \mathcal{F}(\operatorname{Var})$ be an assertion, let $\xi \in \operatorname{free}(\varphi)$ be a free variable of the assertion, and let $\zeta \in \operatorname{Var} \backslash \operatorname{var}(\varphi)$ be a fresh variable. The assertion $\varphi$ holds in a state $s$ under a valuation $\nu$, if and only if the assertion $\varphi[\zeta / \xi]$ holds in $s$ under the valuation $\nu^{\prime}$, such that $\nu^{\prime}(\zeta)=\nu(\xi)$ and $\nu^{\prime}$ coincides with $\nu$ on all other free variables of $\varphi$.

Proofs. Both lemmas are proven by induction on the structure of formulas, as exposed in the grammar in Fig. 3.1. One proves for each derivation that an expression (resp. a formula), has the same value (resp. truth value) in $s$ under $\nu$ or $\nu^{\prime}$, taking into account the variable renaming in the case of the second lemma.

\subsubsection{Global Assertions}

Definition 4.4. A global assertion is a formula from the language $\mathcal{F}(\emptyset)$.

A global assertion is a closed, quantified, flat first-order formula. An example is

$$
\forall p \text { :Person } \forall c \text { : Car }((p . a g e>60 \wedge c=p . c a r) \rightarrow c . c o l o r=\operatorname{Red}) .
$$

Strictly speaking, both a state and a valuation should be used to give a truth value to a global assertion. Yet, since a global assertion is a closed formula, Lemma 4.1 indicates that any valuation can be used, and it is usually omitted.

We thus say that a global assertion $\varphi$ holds in a given state $s$ if and only if this state, together with any valuation, makes the formula valid. We note this $s \models \varphi$. For example, for any state $s$ one has: $s \models \forall p(p$.age $>21 \rightarrow p$.age $\geq 0)$. 


\subsubsection{Assertions Focused on a Rule}

One often needs to express properties of the values in a given state of attributes of the objects matched by a rule. For this purpose, we introduce assertions focused on a rule. These assertions have no other free variable than the variables of the rule, and are well-typed with respect to the rule type constraint.

Definition 4.5. Given a rule $r=(\vec{o}, \vec{T}, g, a)$ with $\vec{o}=\left\{o_{1}, \ldots, o_{m}\right\}$, an assertion $\varphi$ is focused on $r$ if

(i) $\varphi \in \mathcal{F}\left(\left\{o_{1}, \ldots, o_{m}\right\}\right)$, and

(ii) $\varphi$ is well-typed with respect to $(\vec{o}: \vec{T})$.

We then note $\varphi \in \mathcal{F}(r)$.

Given a rule $r$ of arity $m$, an assertion $\varphi$ focused on $r$, and a tuple $\vec{x}=$ $\left(x_{1}, \ldots, x_{m}\right)$ of $m$ variables, we will note $\varphi\left(x_{1}, \ldots, x_{m}\right)$ or $\varphi(\vec{x})$ the assertion $\varphi$ where the variables $o_{1}, \ldots, o_{m}$ have been replaced by the variables $x_{1}, \ldots, x_{m}$, respectively: $\varphi(\vec{x})=\varphi\left[x_{j} / o_{j}\right]_{j=1}^{m}$.

\subsection{Transition Assertions}

While state assertions introduced in the previous section are to be interpreted in a given state, a transition assertion relates attribute values as interpreted in two different states. Typically the initial and final states in an execution.

Transition assertions (such as p.bonus' $>$ p.bonus) involve primed or quoted attribute symbols; they can have free variables or be closed.

The purpose of assertions on transitions between states, or transition assertions, is to express a property on the relationship between two states, such as the initial state and the final state in the execution of a rule or rule program.

Depending on the way one looks at the transition, that is, from the viewpoint of the initial or of the final state, a given property can be expressed in two ways. To this end, we define present forward and backward transition assertions, a concept introduced for example in [MP89].

\subsubsection{Forward Transition Assertions}

Classically [Lam94], we start by extending the set of attribute symbols Attr with new attribute symbols obtained by adding a prime to each attribute symbol in Attr. We note $\mathrm{Attr}^{+}$the extended set:

$$
\operatorname{Attr} \stackrel{+ \text { def }}{=} \operatorname{Attr} \cup\left\{f^{\prime} \mid f \in \operatorname{Attr}\right\} .
$$

We then introduce forward transition assertions as the first-order, flat, possibly quantified formulas as defined by the same grammar exposed in Fig. 3.1 that defines the $\mathcal{F}($ Var $)$ language, but where attribute symbols are taken from $\mathrm{Attr}^{+}$instead of just Attr. For a set $V \subseteq$ Var of variables, we note $\mathcal{F}^{+}(V)$ the set of forward transition assertions in the variables of $V$.

A forward transition assertion is thus a formula that contains primed and unprimed attribute symbols. An example is

$$
\forall p\left(\text { p.age }<60 \wedge \text { p.bonus }{ }^{\prime}>\text { q.bonus }\right) \vee \text { q.age }>21 .
$$


As all of our formulas, a forward transition assertion is to be interpreted by a first-order logic structure and a valuation. Here, the first-order logic structure has to provide an interpretation for both primed and unprimed attribute symbols. This can be achieved with state pairs defined as follows.

Definition 4.6. Given two states $s_{1}$ and $s_{2}$, the state pair based on $s_{1}$ and $s_{2}$, noted $\left\langle s_{1}, s_{2}\right\rangle$, is the first-order logic structure with the domain $\mathcal{D}$ common to both states, and an interpretation function that can offer two interpretations for each attribute symbol in Attr. Other syntactic constructs are interpreted in the way common to both states, defined in Section 4.1.

We say that a forward transition assertion $\varphi$ holds in a state pair $\left\langle s_{1}, s_{2}\right\rangle$ under a valuation $\nu$, or that $\varphi$ holds between $s_{1}$ and $s_{2}$ under $\nu$, if the first-order logic structure $\left\langle s_{1}, s_{2}\right\rangle$ where each unprimed attribute symbol is interpreted as in $s_{1}$ and each primed attribute symbol is interpreted in the same way as the corresponding unprimed attribute symbol in $s_{2}$, together with the valuation $\nu$, make $\varphi$ valid. We note this $\left\langle s_{1}, s_{2}\right\rangle, \nu \models \varphi$.

A forward transition assertion relates initial values of attribute of objects, denoted by unprimed attribute symbols, to their final values, denoted by primed attribute symbols.

Example. The transition assertion

$$
\varphi \equiv \text { p.bonus }{ }^{\prime} \leq \text { p.bonus }+5
$$

is made valid by a state pair $\left\langle s_{1}, s_{2}\right\rangle$ and a valuation $\nu$ that satisfies for example

$$
\nu(p)=\text { Alice } \quad \text { bonus }^{s_{1}}(\text { Alice })=20 \quad \text { bonus }^{s_{2}}(\text { Alice })=23 .
$$

The meaning of $\varphi$ as a transition assertion is that the bonus of the object held by $p$ is not incremented by more than 5 during the transition.

\subsubsection{Backward Transition Assertions}

We define another extension of the set of attribute symbols by adding a quote in front of each attribute symbol in Attr. We note Attr ${ }^{-}$this other extension:

$$
\text { Attr } \stackrel{\text { def }}{=} \operatorname{Attr} \cup\left\{{ }^{\prime} f \mid f \in \operatorname{Attr}\right\} .
$$

The symbol ' $f$ is usually pronounced "old $f$ ".

Backward transition assertions are first-order, flat, possibly quantified formulas as defined by the grammar exposed in Fig. 3.1, but where attribute symbols are taken from $\mathrm{Attr}^{-}$instead of Attr. For a set $V \subseteq$ Var of variables, we note $\mathcal{F}^{-}(V)$ the set of backward transition assertions in the variables of $V$. As mentioned previously, a backward transition assertion is simply another way at looking at the same property as the forward transition assertion obtained by replacing unprimed attribute symbols with primed ones, and quoted ones with unprimed ones.

An example of a backward transition assertion (equivalent to the first example of forward transition assertion) is

$$
\forall p\left(p .^{\prime} \text { age }<60 \wedge \text { p.bonus }>\text { q.'bonus }\right) \vee q .^{\prime} \text { age }>21 .
$$


A backward transition assertion relates initial values of attribute of objects, denoted by quoted attribute symbols, to their final values, denoted by unprimed attribute symbols. We say that a backward transition assertion $\varphi$ holds in a state pair $\left\langle s_{1}, s_{2}\right\rangle$ under a valuation $\nu$, or that $\varphi$ holds between $s_{1}$ and $s_{2}$ under $\nu$, if the first-order logic structure $\left\langle s_{1}, s_{2}\right\rangle$ where each quoted attribute symbol is interpreted in the same way as the corresponding unprimed attribute symbol in $s_{1}$ and each unprimed attribute symbol is interpreted as in $s_{2}$, together with the valuation $\nu$, make it valid. We note this $\left\langle s_{1}, s_{2}\right\rangle, \nu \models \varphi$.

\subsubsection{Global Transition Assertions}

Global (forward or backward) transition assertions are closed transition assertions, that is, formulas in $\mathcal{F}^{+}(\emptyset)$ or $\mathcal{F}^{-}(\emptyset)$. As for other closed assertions, we omit the valuation when interpreting global transition assertions, noting $\left\langle s_{1}, s_{2}\right\rangle \models \varphi$ when the global transition assertion $\varphi$ holds between $s_{1}$ and $s_{2}$.

Examples of global assertion transitions are

$$
\begin{aligned}
& \forall p\left(p . \text { bonus }^{\prime} \leq p . \text { bonus }+2\right) \\
& \forall p \forall c\left(p .^{\prime} \text { cat }=\text { Gold } \wedge c .^{\prime} \text { value }>1000 \rightarrow c . \text { discount }>c .^{\prime} \text { discount }\right) .
\end{aligned}
$$

A frequently used global transition assertion is the one that states the identity between two states, that is, the fact that all attributes have the same values on all objects in both states. Here is this transition assertion, as a forward then as a backward assertion:

$$
\begin{aligned}
& \mathrm{Id}^{+} \stackrel{\text { def }}{=} \bigwedge_{f \in \text { Attr }} \forall o\left(o . f=o . f^{\prime}\right) \\
& \mathrm{Id}^{-} \stackrel{\text { def }}{=} \bigwedge_{f \in \text { Attr }} \forall o(o \cdot f=o . f) .
\end{aligned}
$$

\subsection{Semantics of Assignment}

Assignment has been introduced in Section 3.4; its semantics is defined here, as producing a new state where the assigned attribute is updated. A link is established with transition assertions described in the previous section.

In Section 3.4, we defined the syntax of the assignment of a value to an attribute of an object. The effect of an assignment, when executed in a given state and under a given valuation, is to produce a new state. More generally, an assignment as a statement defines a relation between states.

The state produced by the execution of an assignment coincides with the initial state, except on the interpretation of the attribute that is assigned to, where it coincides with the updated attribute, as defined below. This definition follows the approach of [Mor82], also exposed in detail in [AdBO09, pp. 42-45].

\subsubsection{Update of an Attribute}

Definition 4.7. Given an attribute symbol $f \in$ Attr, a variable $o \in \operatorname{Var}$, and an expression $e \in \mathcal{E}($ Var $)$, the update of $f$ on o by $e$ is the attribute noted $f[o \mapsto e]$, 
and interpreted in a state $s$ and under a valuation $\nu$ by the function $f[o \mapsto e]_{\nu}^{s}$ defined as follows for any $O \in \mathbb{D}$ :

$$
f[o \mapsto e]_{\nu}^{s}(O)= \begin{cases}\mathcal{I}_{s}\left(e[\nu(\xi) / \xi]_{\xi \in \operatorname{Var}}\right) & \text { if } O=\nu(o) \\ f^{s}(O) & \text { otherwise }\end{cases}
$$

An alternate notation for $f[o \mapsto e]$ is $\lambda x$.if $x=o$ then $e$ else $x$. $f$. Note that the equality $x=o$ here is a semantic one, as opposed to a syntactic one: what is to be tested is not whether the variables have the same names, but whether the objects referred to by the variables are in fact the same object or not. This information will be given by the valuation that comes with the state in the interpretation of the updated attribute.

Example. Consider the attribute value, to be updated on the variable $o$ by the expression $e \equiv$ o.value $\times$ p.bonus.

Consider also the following state $s$ and valuation $\nu$ :

$$
\begin{aligned}
& s: \text { bonus }^{s}: \text { Alice } \mapsto 2 \text { value }{ }^{s}: \text { Caddie } \mapsto 50 \\
& \text { Bob } \mapsto 3 \quad \text { Shopper } \mapsto 80 \\
& \text { Carol } \mapsto 4 \quad \text { otherwise } \mapsto \perp \\
& \text { otherwise } \mapsto \perp \\
& \nu: o \mapsto \text { Caddie } \quad p \mapsto \text { Carol } \quad \text { otherwise } \mapsto \text { anything. }
\end{aligned}
$$

The attribute value $[o \mapsto e]$ will be interpreted as follows in $s$ under $\nu$ :

$$
\begin{aligned}
\text { value }[o \mapsto e]_{\nu}^{s}: \text { Caddie } & \mapsto 200 \\
\text { Shopper } & \mapsto 80 \\
\text { otherwise } & \mapsto \perp .
\end{aligned}
$$

\subsubsection{Executing an Assignment}

Consider an assignment $a \equiv$ o.f $:=e$, where $o$ is a variable, $f$ is an attribute symbol, and $e$ is an expression. The free variables in this statement are $o$ plus the free variables of $e$.

The execution of this assignment in a state $s$ under a valuation $\nu$ produces a new state $s^{\prime}$ where:

(i) The domains of $s$ and $s^{\prime}$ are $\mathcal{D}$; the interpretations of all constant symbols and operators are the same in $s$ and $s^{\prime}$.

(ii) The interpretations of all attribute symbols except $f$ are the same in $s$ and $s^{\prime}$. That is, for any $g \in \operatorname{Attr} \backslash\{f\}$ we have $g^{s^{\prime}}=g^{s}$.

(iii) The attribute symbol $f$ is interpreted in $s^{\prime}$ as $f[o \mapsto e]$ is interpreted in $s$ under $\nu$.

We note $s \stackrel{(a, \nu)}{\longrightarrow} s^{\prime}$ the fact that $s^{\prime}$ is the state produced by the execution of the assignment $a$ in $s$ under $\nu$.

Example. Consider the assignment o.value $:=0$.value $\times$ p.bonus, executed in the state $s$ and under the valuation $\nu$ introduced in the previous example. This 
execution produces the state $s^{\prime}$ defined as follows:

$$
\begin{aligned}
s^{\prime}: \text { bonus }^{s^{\prime}}: \text { Alice } & \mapsto 2 & \text { value }^{s^{\prime}}: \text { Caddie } & \mapsto 200 \\
\text { Bob } & \mapsto 3 & \text { Shopper } & \mapsto 80 \\
\text { Carol } & \mapsto 4 & \text { otherwise } & \mapsto \perp . \\
\text { otherwise } & \mapsto \perp & &
\end{aligned}
$$

\subsubsection{Assignment as a Relation Between States}

Given two states $s$ and $s^{\prime}$, we would like to say that there is an execution of an assignment $a$ leading from $s$ to $s^{\prime}$ if there exists a valuation $\nu$, such that $s^{\prime}$ is the state produced by the execution of the assignment $a$ in $s$ under $\nu$. That is,

$$
s \stackrel{a}{\rightarrow} s^{\prime} \quad \text { iff } \quad \text { there exists } \nu \text { s.t. } s \stackrel{(a, \nu)}{\longrightarrow} s^{\prime} .
$$

However, since there is an infinite number of variables and objects, there is an infinite number of valuations, and hence in general an infinite number of states resulting from the execution of an assignment in a given state. Practically, only a finite number of state pairs in the relation that defines the semantics of an assignment are interesting, namely the ones that preserve the non- $\perp$ values of attributes.

To restrict the definition of $s \stackrel{a}{\rightarrow} s^{\prime}$ to only the relevant instances $(a, \nu)$, we need the notion of well-typed valuation, which is introduced in Section 5.2.3. The definition of assignment as a relation between states is therefore given in Section 6.3.1, as part of the semantics of loop-less sequential programs. However the semantics of an assignment given in Section 4.4.2 is already enough to give a meaning to a rule action, as done in the next section.

\subsection{Rule Guard and Action}

Based on the semantics of expressions, formulas, and assignment introduced in the preceding sections of this chapter, the semantics of the guard and action of a rule can be described, respectively, by an assertion on states and by a transition assertion. This description is relied upon in the next chapter, to define what executing a rule or a rule program means.

\subsubsection{Rule Guard}

A rule $r=(\vec{o}, \vec{T}, g, a)$ is defined in Section 3.5 as consisting of a tuple of variables, a tuple of type names, a guard, and an action. The tuple of variables $\vec{o}=$ $\left(o_{1}, \ldots, o_{m}\right)$ and the tuple of type names $\vec{T}=\left(T_{1}, \ldots, T_{m}\right)$ have the same arity $m$. The rule action $a \in \mathcal{A}\left(\left\{o_{1}, \ldots, o_{m}\right\}\right)$ is discussed in the next section.

The rule guard $g \in \mathcal{B}\left(\left\{o_{1}, \ldots, o_{m}\right\}\right)$ is a first-order, flat, non-quantified formula. The rule variables and type names form a type constraint $(\vec{o}: \vec{T})$, for which $g$ has to be well-typed.

It follows from this definition that the guard of a rule is an assertion focused on the rule, as defined in Section 4.2.3: $g \in \mathcal{F}(r)$. 


\subsubsection{Rule Action}

As indicated in Section 3.5.3, the action of a rule is a single assignment. An extended syntax and semantics of rule actions is provided in [BDS12] for sequences of assignments. More generally, a rule action could be extended to a sequential program, as described in the literature [AdBO09], or in Chapters 6 and 7 .

For the sake of simplicity, we consider rule actions made of a single assignment of an attribute of the object held by the first rule variable. That is, in a rule $r=(\vec{o}, \vec{T}, g, a)$ the action is $a \equiv o_{1} \cdot f_{r}:=e_{r}$ with $f_{r} \in \alpha\left(T_{1}\right)$ and $e_{r} \in \mathcal{E}\left(\left\{o_{1}, \ldots, o_{m}\right\}\right)$. The rule action $a$ is well-typed for the rule type constraint $(\vec{o}: \vec{T})$.

The semantics of the rule action is the one described in Section 4.4: the state $s^{\prime}$ that results from the execution of $a$ in a state $s$ and under a valuation $\nu$ interprets all other attributes than $f_{r}$ in the same way as $s$, and interprets $f_{r}$ as $s$ interprets the updated attribute $f_{r}\left[o_{1} \mapsto e_{r}\right]$.

This relationship $s \stackrel{(a, \nu)}{\longrightarrow} s^{\prime}$ can be described by a transition assertion linking the initial and final states $s$ and $s^{\prime}$ under the valuation $\nu$ :

$$
\left\langle s, s^{\prime}\right\rangle, \nu \models \forall o\left(o . f_{r}^{\prime}=\left(\lambda x \text {.if } x=o_{1} \text { then } e_{r} \text { else } x . f_{r}\right)(o)\right) \wedge \bigwedge_{f \in \operatorname{Attr} \backslash\left\{f_{r}\right\}} \forall o\left(o . f^{\prime}=o . f\right)
$$

which can be rewritten as $\left\langle s, s^{\prime}\right\rangle, \nu \models \hat{a}$, where $\hat{a}$ is the transition assertion for the action $a$, as defined below.

Definition 4.8. Given a rule $r=(\vec{o}, \vec{T}, g, a)$ with $\vec{o}=\left(o_{1}, \ldots, o_{m}\right)$ and $a \equiv$ $o_{1} \cdot f_{r}:=e_{r}$, the transition assertion for the action $a$ of the rule $r$ is

$$
\hat{a} \stackrel{\text { def }}{\equiv} o_{1} \cdot f_{r}^{\prime}=e_{r} \wedge \forall o\left(o \neq o_{1} \rightarrow o . f_{r}^{\prime}=o . f_{r}\right) \wedge \bigwedge_{f \in \operatorname{Attr} \backslash\left\{f_{r}\right\}} \forall o\left(o . f^{\prime}=o . f\right) .
$$

This transition assertion is a forward transition assertion in the rule variables: $\hat{a} \in \mathcal{F}^{+}(\operatorname{var}(r))$. It is by construction well-typed for the rule type constraint $(\vec{o}: \vec{T})$.

Example. Consider the following rule:

$$
r(p: \text { Person }): \text { p.age }>10 \rightarrow \text { p.bonus }:=\text { p.age }-10
$$

In a setting where the attribute symbols defined by our type system are age and bonus, the transition assertion for the action of this rule is

$$
\text { p.bonus }{ }^{\prime}=\text { p.age }-10 \wedge \forall o\left(o \neq p \rightarrow \text { o.bonus }{ }^{\prime}=\text { o.bonus }\right) \wedge \forall o\left(\text { o.age } e^{\prime}=\text { o.age }\right) .
$$




\section{Chapter 5}

\section{Operational Semantics of Rule Programs}

In Business Rules Management Systems, and more generally in production systems, a rule program is executed by a machine called a rule engine, on a set of objects called the working memory [BFKM85]. Execution of a rule program consists of a sequence of applications of rule instances, that is, the execution of the rule actions on the objects that make the rule guards valid.

In this chapter, we build on the previous two chapters to define the working memory, rule instances, and rule execution, and finally an abstract machine that describes the execution of a rule program. The operational semantics that we define differs from previous formalizations of production systems [FL92, CKMM04, dSMHP10], in that we differentiate eligibility and applicability of a rule instance. The applicability of a rule instance relates to whether the rule guard is valid on the objects in the instance, whereas the eligibility of a rule instance is determined by the rule instances executed so far by the rule engine. This distinction between eligibility and applicability allows us to describe other execution strategies than the historical Rete algorithm.

After an informal presentation of the notions at stake in the execution of rule programs, we formally define the abstract machine that models rule engines.

\subsection{Rule Program Execution, Informally}

We start the chapter with an informal description of the execution of a rule program by a rule engine. This description is the occasion to give an overview of the concepts at stake and of their interactions. Its aim is both to present the rule programming paradigm to readers unfamiliar to it, and to help readers more familiar with the implementation of rule engines to connect their experience with the subsequent formal description.

\section{Declarative programs}

Rule programs belong to the category of declarative programming paradigms, in the sense that the text of a rule program is a collection of independent statements 
(the rules), and does not explicit the control flow, that is, how the statements are to be combined upon execution of the program.

Rule programs share this aspect with parallel programs, where the processes composing the program are combined upon execution by a scheduler that is not explicit in the program. A given parallel program might even admit distinct sets of executions if it is run using different schedulers.

In contrast, imperative programs contain in their texts the control structures that dictate the combination of their statements.

\section{Rule engine}

The rule engine is the mechanism that conducts the execution of a rule program. Like the schedulers of parallel programs, a rule engine can adopt variations in its behavior. These variations are collectively referred to as the execution strategy, and basically arbitrate between several possible sequences of rule executions.

On a given data set and under a given execution strategy, a rule program will in practice always exhibit the same execution. This behavior is mandatory for actual rule engines, on which the logic of business-critical applications is based, so that rule programs have reproducible executions. It is however interesting, from the viewpoint of program analysis and verification, to allow a degree of nondeterminism in the execution strategy. It is the case with the execution strategies described in Sections 5.5 and 5.6: when using these abstract execution strategies, a rule program can have several possible executions on a given data set.

\section{Working memory}

A specificity of rule programs is that not only their texts have a declarative form, but the data on which they are executed as well.

When an imperative or a parallel program is executed on a given data set, the components of the programs (statements, processes) are instantiated: each variable is given a unique initial value taken from the data set. The instances of the components are then combined for execution, either linearly or in parallel.

In contrast, when a rule program is executed on a given data set, the rule engine creates multiple instances of each rule. It then combines all the rule instances according to its execution strategy. The data set on which a rule program is executed is a collection of objects, and is called the working memory. It is formally defined in Section 5.2 below, and rule instances are defined in Section 5.3.1.

This way of feeding data into a program by saturation on a working memory is not the classical way of executing other kinds of program. It is rather typical for paradigms described in Section 2.1, such as deductive databases and rule programs. However, execution by saturation is general enough to be defined for parallel programs and nondeterministic programs, as we do in the next chapter.

\section{Execution}

In Fig. 5.1, we summarize the work performed by a rule engine. Of course, the mechanisms given in this figure and in the description below are implemented in an optimized manner by the rule engines found in actual Business Rules Management Systems. 


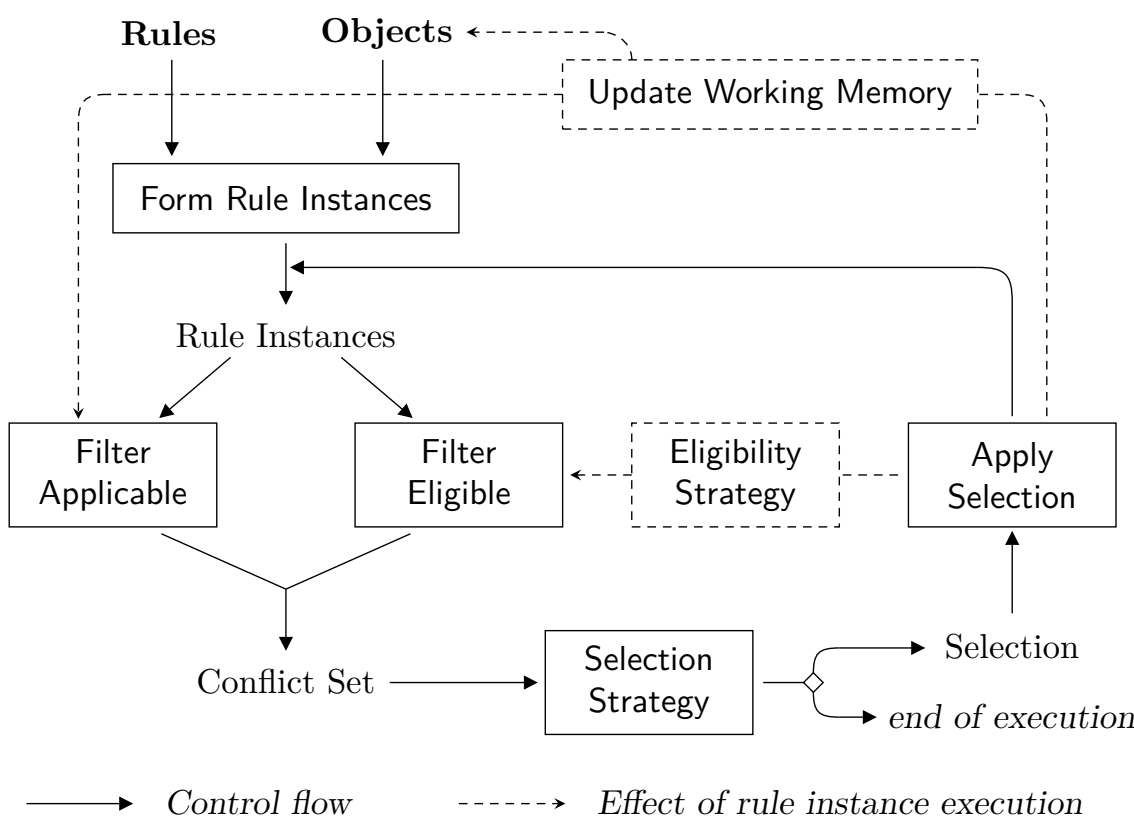

Figure 5.1: Execution of a rule program by a rule engine.

The execution of a rule program is composed of a series of rule instance applications. The role of the rule engine is to combine the rules in the rule program and the objects in the working memory to form rule instances, then to assess their applicability in the current state, and to evolve this current state by executing the actions of chosen rule instances. All these notions are exposed in detail below and are formally defined later in this chapter.

The first task of the rule engine is to form rule instances by assembling the rules in the program and the objects found in working memory. The resulting set of rule instances is filtered in two independent ways. On the one hand, the applicability of each rule instance in the current state is assessed. This amounts to determining, for each rule instance, the truth value of the guard of the rule on the objects in the instance. On the other hand, the eligibility of each rule instance is tested.

The eligibility of rule instances is a notion used by rule engines to avoid trivial loops due to the repeated execution of rule instances whose application produces a state where the rule instance is still applicable. An example of such a trivial loop is given by the rule $r(p:$ Person $):$ p.age $>21 \rightarrow$ p.bonus $:=10$. If an instance of this rule is applicable once, it remains applicable after it has been applied. Defining that at some point the rule instance is not eligible for execution although its guard might hold, is a way to avoid its endless application. Knowing which rule instances are eligible at a given moment is defined by the eligibility strategy of the engine.

Once the applicability and eligibility of all rule instances have been independently assessed, the conflict set is formed with the rule instances that are both applicable and eligible.

The next task of the rule engine is to apply its selection strategy (also called 
the conflict set resolution strategy), to choose in the conflict set a rule instance that shall be applied. In Section 5.5, we will see that a selection strategy is often based on an order defined on rule instances. As a consequence, it is common to implement the conflict set as an ordered list; it is then called the agenda. The rule instance chosen by the selection strategy is either named the selection or, as a result of the previous remark, the head of the agenda.

It can happen however that there is no head of the agenda, either because the conflict set is empty, that is, no rule instance is both applicable and eligible, or because the selection strategy retained none of them. In this case, the execution of the rule program terminates, with the current state of the engine as the resulting state of the execution.

If there is a head of the agenda, then the rule engine executes the action of this rule instance. The effect of this execution is twofold. Firstly, it updates the current state with the state that results from the execution of the statements in the rule action. That is in our setting, the assignment of a new value to an attribute of an object. This update might have an effect on the applicability of some rule instances. More generally the action of a rule might also insert new objects into the working memory, or remove objects from it, which might additionally affect the list of rule instances to be filtered. Secondly, it updates the eligibility of the rule instances according to the engine's eligibility strategy. The rule engine then starts again, updating the conflict set, selecting a new head of the agenda, etc.

\section{Properties of executions}

As noted, the execution of a rule program is subject to the choices implemented in the selection and eligibility strategies. These strategies are formally defined further below in this chapter, and several examples of each are given. They are collectively referred to as the execution strategy of the engine.

The selection strategy mainly controls whether only one, or several, or even many, executions of a rule program are possible on a given working memory. As already mentioned, implementations of rule engines must ensure that the execution of a rule program on a given working memory can be repeated. More generally, similar properties as for schedulers of parallel programs, such as fairness, are involved for selection strategies.

The eligibility strategy mainly influences the termination of rule programs, with some strategies having the property of ensuring the termination of all rule programs.

\subsection{Working Memory}

In the formal framework introduced in Chapter 4, the set of objects is infinite. The working memory gathers the finite subset of objects on which the rule program is to be executed.

In this section, we see that in general the working memory depends on the state considered. However, under simple hypotheses that hold during a rule program execution, the working memory depends only on the types used in the rule program. Furthermore, it is preserved by the execution of a well-typed assignment (as found in rule actions). 
We take advantage of these properties in the next chapter, to state that a working memory remains constant throughout a rule program execution.

We informally described the working memory earlier, as the set of objects on which a rule program is executed. In this section, we establish a link between the (infinite) set of all objects and the type system underlying our formalism. These definitions allow us to consider objects as instances of types, and to define the working memory as the collection of all instances of all types. In addition, we will see that the working memory is finite and constant over rule program executions.

\subsubsection{Type-System Compliant States}

In Chapter 3 , we fixed and defined a type system $\mathcal{T}=($ Types, $\alpha)$, which introduces the set Types of type names and the type definition function $\alpha$ that maps type names onto sets of attribute symbols.

In Section 3.3.2, we also defined a type constraint as being a partial function associating a type name with each variable in its domain. We additionally defined well-typed expressions and formulas for a type constraint $\tau$, as those including only attribute references (of the form o.f) in which the attribute symbol belongs to the definition of the type associated with the variable by the type constraint (that is, $f \in \alpha(\tau(o))$ ).

Finally, in Section 3.5, we introduced rules of the form $r=(\vec{o}, \vec{T}, g, a)$, where the tuple of variables $\vec{o}$ and the tuple of type names $\vec{T}$ constitute a type constraint noted $(\vec{o}: \vec{T})$, for which $g$ and $a$ must be well-typed.

We define below first the attribute span of an object in a state, then the notion of a type-system compliant state.

Definition 5.1. Given an object $O \in \mathbb{D}$ and a state $s$, the attribute span of $O$ in $s$, noted $\alpha_{s}(O)$, is the set of attribute symbols for which $O$ has a non- $\perp$ value in $s$. That is,

$$
\alpha_{s}(O)=\left\{f \in \operatorname{Attr} \mid f^{s}(O) \neq \perp\right\}
$$

The attribute span of an object is a symmetric notion to the domain of an attribute symbol introduced in Section 4.1.3. It also corresponds to what is sometimes called the effective type of an object.

Definition 5.2. A state $s$ is $\mathcal{T}$-compliant, or compliant with the type system $\mathcal{T}$, if all objects with a non-empty attribute span in $s$ match the definition of a type in the type system. That is, for any $O \in \mathbb{D}$, either $\alpha_{s}(O)=\emptyset$, or there exists $T \in$ Types such that $\alpha_{s}(O)=\alpha(T)$.

Example. Examples of compliant and non-compliant states are included in the example of the next section.

In states that are $\mathcal{T}$-compliant, we can consider objects as instances of types, as defined below. 


\subsubsection{Objects as Instances of Types}

Definition 5.3. Given a type name $T \in$ Types and a $\mathcal{T}$-compliant state $s$, the instances of $T$ in $s$, noted $\mathcal{M}_{s}(T)$, are the objects whose attribute span in $s$ matches the definition of $T$. That is,

$$
\mathcal{M}_{s}(T)=\left\{O \in \mathbb{D} \mid \alpha_{s}(O)=\alpha(T)\right\} .
$$

From the requirement stated in Section 4.1.3 that attribute symbols must have a finite domain in any state, it follows that the set of instances of a type is finite.

In addition, we stated in Section 3.3.2 the requirement for type definitions to be disjoint, that is, for any two distinct types names $T_{1}, T_{2} \in$ Types, we have $\alpha\left(T_{1}\right) \cap \alpha\left(T_{2}\right)=\emptyset$. (We noted there that this does not reduce our expressivity, and is usually made transparent to the user through some mangling process performed by the language compiler.) It follows from this requirement that the sets of instances of two distinct types are disjoint as well.

As a consequence, if the attribute span of an object in a given state matches the definition of a type, this type is unique. We can therefore say that $T$ is the type of an object $O$ in a $\mathcal{T}$-compliant state $s$, if $O \in \mathcal{M}_{s}(T)$.

Note that, for a $\mathcal{T}$-compliant state $s$ and a valuation $\nu$, one has $s, \nu \models o: T$ if and only if $\nu(o) \in \mathcal{M}_{s}(T)$. Also, to assess whether $s, \nu \models \forall \xi: T \varphi$ holds, it suffices to assess whether $s, \nu^{\prime} \models \varphi$ holds for $\nu^{\prime}(\xi) \in \mathcal{M}_{s}(T)$ and $\nu^{\prime}$ coinciding with $\nu$ on the other variables.

Definition 5.4. Given a state $s$, the working memory in $s$ is the set of objects with at least one non- $\perp$ attribute value in $s$. We note it $\mathcal{M}_{s}$ and we thus have

$$
\mathcal{M}_{s} \stackrel{\text { def }}{=}\left\{O \in \mathbb{D} \mid \text { there exists } f \in \operatorname{Attr} \text { s.t. } f^{s}(O) \neq \perp\right\} .
$$

The fact that the working memory is finite in any state derives from the requirement that attribute symbols must have a finite domain in any state. It can also be derived from the following equivalent definitions of the working memory in a $(\mathcal{T}$-compliant where required) state $s$ :

$$
\begin{array}{ll}
\mathcal{M}_{s}=\bigcup_{f \in \operatorname{Attr}} \operatorname{Dom}_{s}(f) & \text { - union of the domains of all attributes; } \\
\mathcal{M}_{s}=\left\{O \in \mathbb{O} \mid \alpha_{s}(O) \neq \emptyset\right\} & \text { - all objects with a non-empty attribute span; } \\
\mathcal{M}_{s}=\biguplus_{T \in \text { Types }} \mathcal{M}_{s}(T) & \text { - disjoint union of the instances of all types. }
\end{array}
$$

Example. Let us consider the setting depicted in Fig. 5.2.

We have

$$
\begin{aligned}
\alpha_{s}(\text { Alice }) & =\{\text { age }, \text { car }\} & \alpha_{s}(\text { Alfa }) & =\{\text { color }, \text { mileage }\} \\
\alpha_{s}(\text { Bob }) & =\{\text { age }, \text { car }\} & \left.\alpha_{s} \text { (Beetle }\right) & =\{\text { color }, \text { mileage }\} .
\end{aligned}
$$

Therefore $s$ is $\mathcal{T}$-compliant and we have

$$
\begin{array}{rlrl}
\mathcal{M}_{s}(\text { Person }) & =\{\text { Alice, Bob }\} & \mathcal{M}_{s}(\text { Car })=\{\text { Alfa }, \text { Beetle }\} \\
\mathcal{M}_{s} & =\{\text { Alice, Bob, Alfa, Beetle }\}
\end{array}
$$




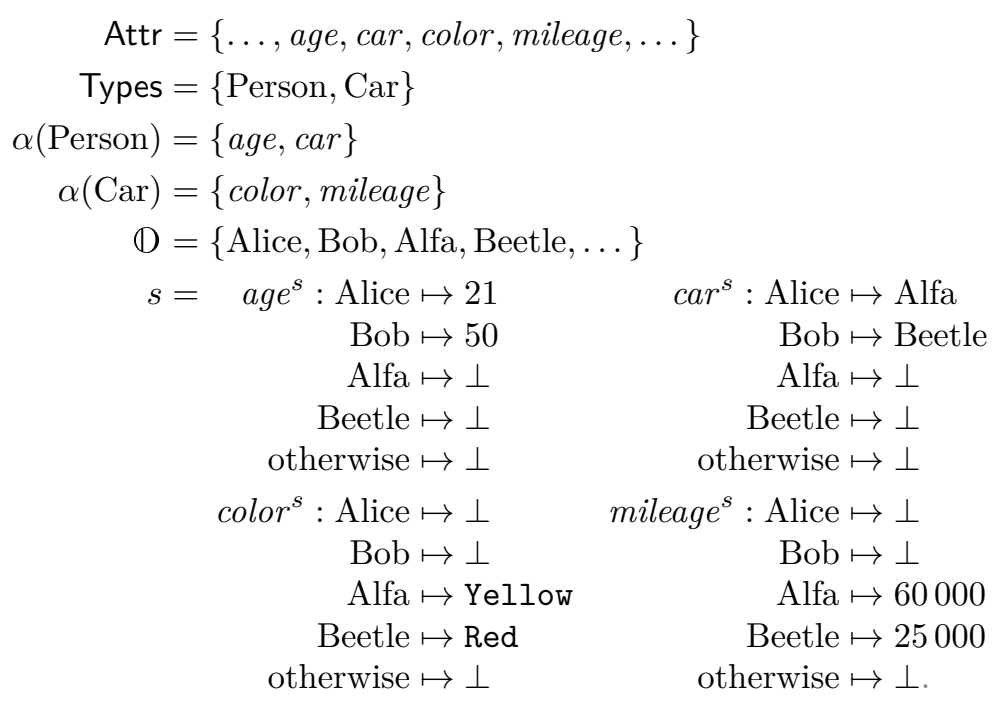

Figure 5.2: Attribute span, $\mathcal{T}$-compliant state, type instances.

The state $s$ would not have been $\mathcal{T}$-compliant if, for example, we had defined $\operatorname{age}^{s}(\mathrm{Alfa})=12$ or mileage $e^{s}($ Beetle $)=\perp$.

\subsubsection{Preservation Properties}

The preservation properties below involve the execution of an assignment. They will be extended to the execution of a rule, and of a rule program, once these are defined. For all these properties the lemma below is crucial: it states the preservation of attribute spans when executing an assignment under a well-typed valuation, which we first define.

Definition 5.5. Given a type constraint $\tau$ and a $\mathcal{T}$-compliant state $s$, a valuation $\nu$ is well-typed for $\tau$ in $s$ if $\nu$ maps each variable of the domain of $\tau$ onto an instance of the type associated with the variable by $\tau$. That is,

$$
\text { for all } \xi \in \operatorname{Dom}(\tau) \text { one has } \nu(\xi) \in \mathcal{M}_{s}(\tau(\xi)) \text {. }
$$

Example. In the context of the previous example, consider the variables $p$ and $c$, and the following valuation and type constraint:

$$
\begin{array}{llll}
\nu: & p \mapsto \text { Alice } & c \mapsto \text { Beetle } & \text { otherwise } \mapsto \text { anything } \\
\tau: & p \mapsto \text { Person } & c \mapsto \text { Car. } &
\end{array}
$$

This valuation $\nu$ is well-typed for $\tau$ in $s$.

Since the set of instances of a type is finite, there is only a finite number of well-typed valuations for a type constraint with a finite domain, in a given state (up to the values given by the valuations to variables outside of the type constraint domain). 
Lemma 5.1. Let $s$ be a $\mathcal{T}$-compliant state, $\tau$ be a type constraint, and $\nu$ be a valuation well-typed for $\tau$ in $s$. Let also $a \in \mathcal{A}(\operatorname{Dom}(\tau))$ be an assignment well-typed for $\tau$, and $s^{\prime}$ be the state that results from the execution of $a$ in $s$ under $\nu$.

Then the attribute span of any object is the same in $s^{\prime}$ as in $s$, that is, for any $O \in \mathbb{D}$ one has $\alpha_{s^{\prime}}(O)=\alpha_{s}(O)$.

Proof. Let the domain of the type constraint $\tau$ be $\operatorname{Dom}(\tau)=\left\{o_{1}, \ldots, o_{m}\right\}$. Consider an object $O \in \mathbb{D}$ and assume that $a \equiv o_{1} . f:=e$. What follows derives from the semantics of assignment as defined in Section 4.4.

If $O \neq \nu\left(o_{1}\right)$ then for any attribute symbol $h \in$ Attr one has $h^{s^{\prime}}(O)=h^{s}(O)$. As a result one has $\alpha_{s^{\prime}}(O)=\alpha_{s}(O)$.

If $O=\nu\left(o_{1}\right)$ then $f^{s^{\prime}}(O)=\mathcal{I}_{s}\left(e\left[\nu\left(o_{j}\right) / o_{j}\right]_{j=1}^{m}\right)$. Since $e$ is well-typed for $\tau$ and $\nu$ is well-typed for $\tau$ in $s$, one has $f^{s^{\prime}}(O) \neq \perp$. On the other hand, since $a$ is well-typed for $\tau$ one has $f \in \alpha\left(T_{1}\right)$, and since $\nu$ is well-typed for $\tau$ in $s$ one has $\alpha_{s}(O)=\alpha\left(T_{1}\right)$, and hence $f \in \alpha_{s}(O)$, that is, $f^{s}(O) \neq \perp$. In addition, for any $h \in \operatorname{Attr} \backslash\{f\}$ one has $h^{s^{\prime}}(O)=h^{s}(O)$. As a result one has $\alpha_{s^{\prime}}(O)=\alpha_{s}(O)$.

It results directly from this lemma that an assignment preserves the properties for states to be type-system compliant and for valuations to be well-typed; it also preserves the instances of types and the working memory.

Proposition 5.2. Let $s$ be a $\mathcal{T}$-compliant state, $\tau$ be a type constraint, and $\nu$ be a valuation well-typed for $\tau$ in $s$. Let also $a \in \mathcal{A}(\operatorname{Dom}(\tau))$ be an assignment well-typed for $\tau$, and $s^{\prime}$ be the state that results from the execution of $a$ in $s$ under $\nu$.

Then $s^{\prime}$ is $\mathcal{T}$-compliant, and $\nu$ is well-typed for $\tau$ in $s^{\prime}$. In addition, the set of instances of any type is the same in $s$ and in $s^{\prime}$; the working memory is the same in $s$ and in $s^{\prime}$.

\subsection{Rule Execution}

Rules are the building blocks of rule programs. Yet a rule is text, whereas the execution of a rule program is the combination of instances of the rules on the objects from the working memory. The actual building blocks of a rule program execution are rule instances, in that they are applicable or not, and they actually produce an effect when applied. Rule execution is defined in this section by building on these concepts.

A rule is a component of a rule program. As such, its instances are combined by the rule engine to form an execution of the rule program. The rule as a statement is also interesting from the viewpoint of the relation that it establishes between states.

In this section we define rule instances, when they are applicable and how they are applied; we also define the effect of executing a rule.

\subsubsection{Rule Instance}

Definition 5.6. Given a rule $r$ of arity $m$, a rule instance $R=\left(r, O_{1}, \ldots, O_{m}\right)$ is a $(m+1)$-tuple made of the rule $r$ and $m$ objects from $\mathbb{D}$. The objects 
$O_{1}, \ldots, O_{m}$ are not necessarily pairwise distinct. We say that $R$ is an instance of rule $r$ on the objects $O_{1}, \ldots, O_{m}$.

The rule instance $\left(r, O_{1}, \ldots, O_{m}\right)$ is also sometimes noted $(r, \vec{O})$, with $\vec{O}=$ $\left(O_{1}, \ldots, O_{m}\right)$.

In addition, given a rule $r=(\vec{o}, \vec{T}, g, a)$ with $\vec{o}=\left(o_{1}, \ldots, o_{m}\right)$, and a valuation $\nu$, we note $(r, \nu)$ the rule instance $\left(r, \nu\left(o_{1}\right), \ldots, \nu\left(o_{m}\right)\right)$. In particular, given two valuations $\nu$ and $\nu^{\prime}$ that coincide on $\vec{o}$, the notations $(r, \nu)$ and $\left(r, \nu^{\prime}\right)$ refer to the same rule instance.

\subsubsection{Applicability of a Rule Instance}

We say that a rule instance is applicable, or that the rule is applicable to the objects in the instance, when the guard of the rule is valid on the objects. As defined below, this depends on the state considered.

Definition 5.7. Given a rule instance $R=\left(r, O_{1}, \ldots, O_{m}\right)$ with $r=(\vec{o}, \vec{T}, g, a)$ and a state $s, R$ is applicable in $s$ if for any valuation $\nu$ that maps each variable $o_{j}$ onto the object $O_{j}$ for $j=1, \ldots, m$, one has $s, \nu \models g$.

From Lemma 4.1, we know that if suffices to find one valuation $\nu$ that maps each $o_{j}$ onto $O_{j}$ for $j=1, \ldots, m$, and such that $s, \nu \models g$, to decide that the rule instance is applicable in $s$. Conversely, it suffices to find one valuation $\nu$ that maps each $o_{j}$ onto $O_{j}$ for $j=1, \ldots, m$, and such that $s, \nu \not \models g$, to know that the rule instance is not applicable.

Example. The rule $r$ below states that, provided the customer is old enough, a tenth of the value of his cart should be added to his bonus.

$$
r(p: P, c: C): p . a g e \geq 50 \& p=c . o w n e r-p \cdot b o n u s:=p \cdot b o n u s+c . v a l u e / 10
$$

In this rule, the variables are $(p, c)$ and the guard is $p . a g e \geq 50 \wedge p=c . o w n e r$.

Consider the following setting:

$$
\begin{aligned}
& \mathbb{D}=\{\text { Alice }, \text { Bob }, \text { Caddie }, \ldots\} \\
& \operatorname{Attr}(\mathcal{T})=\{\text { age, bonus, owner, value }\} \\
& s_{1}=\quad a g e^{s_{1}}: \text { Alice } \mapsto 61 \\
& \text { Bob } \mapsto 80 \\
& \text { otherwise } \mapsto \perp \\
& \text { owner }^{s_{1}} \text { : Caddie } \mapsto \text { Alice value }{ }^{s_{1}} \text { : Caddie } \mapsto 500 \\
& \text { otherwise } \mapsto \perp \quad \text { otherwise } \mapsto \perp \text {. }
\end{aligned}
$$

In this setting the rule instance ( $r$, Alice, Caddie) is applicable in $s_{1}$, since $s_{1}, \nu \models$ p.age $\geq 50 \wedge p=c$.owner for any valuation $\nu$ that maps $p$ onto Alice and $c$ onto Caddie. On the other hand, the rule instance ( $r$, Bob, Caddie) is not applicable in $s_{1}$ because owner ${ }^{s_{1}}$ (Caddie) $\neq$ Bob.

\subsubsection{Application of a Rule Instance}

In a state where a rule instance is applicable, the application of the rule instance produces a new state, defined below. The application of a rule instance in a state where it is not applicable is not defined. 
Definition 5.8. Given a state $s$, a rule $r=(\vec{o}, \vec{T}, g, a)$, and a rule instance $R=\left(r, O_{1}, \ldots, O_{m}\right)$ applicable in $s$, the state that results from the application of $R$ in $s$ is the state $s^{\prime}$, such that $\left\langle s, s^{\prime}\right\rangle, \nu \models \hat{a}$ for any valuation $\nu$ that maps each variable $o_{j}$ onto the object $O_{j}$ for $j=1, \ldots, m$.

Let us remind the definition given in Section 4.5 of the transition assertion $\hat{a}$ for the action of a rule $r=(\vec{o}, \vec{T}, g, a)$ with $a \equiv o_{1} . f_{r}:=e_{r}$ :

$$
\hat{a} \stackrel{\text { def }}{\equiv} o_{1} \cdot f_{r}^{\prime}=e_{r} \wedge \forall o\left(o \neq o_{1} \rightarrow o . f_{r}^{\prime}=o . f_{r}\right) \wedge \bigwedge_{f \in \operatorname{Attr} \backslash\left\{f_{r}\right\}} \forall o\left(o . f^{\prime}=o . f\right) .
$$

Example. Applying the rule instance ( $r$, Alice, Caddie) from the previous example in the state $s_{1}$, results into a state $s_{2}$ defined by the following transition assertion for any $\nu$ such that $\nu(p)=$ Alice and $\nu(c)=$ Caddie:

$$
\begin{aligned}
\left\langle s_{1}, s_{2}\right\rangle, \nu \models & p . \text { bonus }^{\prime}=\text { p.bonus }+ \text { c.value } / 10 \\
& \wedge \forall o\left(o \neq p \rightarrow \text { o.bonus }{ }^{\prime}=\text { o.bonus }\right) \wedge \bigwedge_{f \in \operatorname{Attr} \backslash\{\text { bonus }\}} \forall o\left(o . f^{\prime}=o . f\right) .
\end{aligned}
$$

This defines $s_{2}$ as follows:

$$
\begin{aligned}
\text { age }^{s_{2}}: \text { Alice } & \mapsto 61 \\
\text { Bob } & \mapsto 80 \\
\text { otherwise } & \mapsto \perp \\
\text { owner }^{s_{2}}: \text { Caddie } & \mapsto \text { Alice } \\
\text { otherwise } & \mapsto \perp
\end{aligned}
$$

$$
\begin{aligned}
\text { bonus }^{s_{2}}: \text { Alice } & \mapsto \mathbf{8 0} \\
\text { Bob } & \mapsto 10 \\
\text { otherwise } & \mapsto \perp \\
\text { value }^{s_{2}}: \text { Caddie } & \mapsto 500 \\
\text { otherwise } & \mapsto \perp .
\end{aligned}
$$

The transition assertion $\hat{a}$ defines the state $s^{\prime}$ uniquely for a given state $s$ and a given valuation $\nu$. By Lemma 4.1 again, the transition assertion also uniquely defines $s^{\prime}$ for a given $s$ and any valuation that coincides with $\nu$ on the free variables of $\hat{a}$. Note that each of the conjuncts in $\hat{a}$ has a different set of free variables:

- The free variables in $o_{1} . f_{r}^{\prime}=e_{r}$ are $\left\{o_{1}\right\} \cup \operatorname{var}\left(e_{r}\right)$, which can span on the whole $\left\{o_{1}, \ldots, o_{m}\right\}$.

- The free variables in $\forall o\left(o \neq o_{1} \rightarrow o . f_{r}^{\prime}=o . f_{r}\right)$ are limited to $\left\{o_{1}\right\}$.

- Finally, $\bigwedge_{f \in \operatorname{Attr} \backslash\left\{f_{r}\right\}} \forall o\left(o . f^{\prime}=o . f\right)$ has no free variables.

This implies that, when $s^{\prime}$ is the state that results from the application of the rule instance $\left(r, O_{1}, \ldots, O_{m}\right)$ in $s$, one has in particular

$$
\left\langle s, s^{\prime}\right\rangle \models \bigwedge_{f \in \operatorname{Attr} \backslash\left\{f_{r}\right\}} \forall o\left(o . f^{\prime}=o . f\right)
$$

which depends only on the rule $r$, and not on the objects $O_{1}, \ldots, O_{m}$ in the rule instance; and

$$
\left\langle s, s^{\prime}\right\rangle, \nu \models \forall o\left(o \neq o_{1} \rightarrow o \text {. } f_{r}^{\prime}=o . f_{r}\right) \quad \text { for any } \nu \text { that maps } o_{1} \text { onto } O_{1}
$$

which depends only on $r$ and $O_{1}$. 


\subsubsection{Execution of a Rule Instance}

We have just defined the application of a rule instance, which is possible only when the rule instance is applicable. The execution of a rule instance covers in addition the case where the rule instance is not applicable.

Definition 5.9. Given a state $s$, and a rule instance $R$, the state that results from the execution of $R$ in $s$ is the state that results from the application of $R$ in $s$ if $R$ is applicable in $s$, or $s$ itself otherwise.

Considering a rule instance $\left(r, O_{1}, \ldots, O_{m}\right)$ with $r=(\vec{o}, \vec{T}, g, a)$, and a valuation $\nu$ such that $\nu\left(o_{j}\right)=O_{j}$ for $j=1, \ldots, m$, the definition above means that the state $s^{\prime}$ is defined as follows:

(i) if $s, \nu \models g$, then $\left\langle s, s^{\prime}\right\rangle, \nu \models \hat{a}$;

(ii) if $s, \nu \models \neg g$, then $s^{\prime}=s$;

This definition is correct because $s, \nu \not \models g$ is equivalent to $s, \nu \models \neg g$, according to the interpretation defined in Section 4.1; and because Lemma 4.1 prevents us from having both $s, \nu \models g$ for some $\nu$ such that $\nu\left(o_{j}\right)=O_{j}$ for $j=1, \ldots, m$, and $s, \nu \models \neg g$ for some others.

The execution of a rule instance in a given state thus always leads to one resulting state, whether the rule instance is applicable or not.

Given two states $s$ and $s^{\prime}$, and a rule instance $R$ (applicable in $s$ or not), we note $s \stackrel{R}{\rightarrow} s^{\prime}$ the fact that $s^{\prime}$ the state that results from the execution of $R$ in $s$.

Example. Considering the rule $r$ and the states $s_{1}$ and $s_{2}$ introduced in the previous examples, we have

$$
\begin{gathered}
s_{1} \stackrel{(r, \text { Alice,Caddie })}{\longrightarrow} s_{2} \\
s_{1} \stackrel{(r, \text { Bob,Caddie })}{\longrightarrow} s_{1} .
\end{gathered}
$$

\subsubsection{Executions of a Rule}

A rule is executed during the execution of a rule program by the application of one of its instances, if it is applicable. If we consider all the possible executions of instances of a rule from a given state, we obtain a description of the effect of this rule on the state. For the instances of the rule that are applicable in the state, the effect will be to produce a new state; and for the instances that are not applicable, the effect will be to remain in the same state.

To discard trivially inapplicable instances, we will only consider rule instances that are well-typed in the initial state of execution, as defined below.

Definition 5.10. Given a rule $r=(\vec{o}, \vec{T}, g, a)$ and a $\mathcal{T}$-compliant state $s$, the set of well-typed object tuples for $r$ in $s$ is the Cartesian product of the sets of instances of the types constraining the rule variables, that is,

$$
\mathcal{M}_{s}^{(r)} \stackrel{\text { def }}{=} \mathcal{M}_{s}\left(T_{1}\right) \times \ldots \times \mathcal{M}_{s}\left(T_{m}\right) .
$$

In addition, the rule instance $\left(r, O_{1}, \ldots, O_{m}\right)$ is well-typed in $s$ if $\left(O_{1}, \ldots, O_{m}\right)$ is a well-typed object tuple for $r$ in $s$. 
Given a valuation $\nu$, we have introduced in Section 5.3.1 the notation $(r, \nu)$ as an equivalent to $\left(r, \nu\left(o_{1}\right), \ldots, \nu\left(o_{m}\right)\right)$. We have also defined in Section 5.2.3 the notion of a valuation well-typed for a type constraint in a given state. One can note that a rule instance $(r, \nu)$ is well-typed in a state $s$ if and only if $\nu$ is well-typed for $(\vec{o}: \vec{T})$ in $s$.

Definition 5.11. Given a rule $r$ and two states $s$ and $s^{\prime}$, there is an execution of $r$ leading from $s$ to $s^{\prime}$ if there exists an instance $R$ of $r$ well-typed in $s$, such that $s^{\prime}$ results from the execution of $R$ in $s$. That is,

$$
s \stackrel{r}{\rightarrow} s^{\prime} \quad \text { iff } \quad \text { there exists } \vec{O} \in \mathcal{M}_{s}^{(r)} \text { s.t. } s \stackrel{(r, \vec{O})}{\longrightarrow} s^{\prime} .
$$

We also say that $s^{\prime}$ results from the execution of $r$ in $s$.

Example. Considering again the rule $r$ and the states $s_{1}$ and $s_{2}$ introduced in the previous examples, one has $s_{1} \stackrel{r}{\rightarrow} s_{1}$ and $s_{1} \stackrel{r}{\rightarrow} s_{2}$, which derive from the executions of the rule instances ( $r$, Bob, Caddie) and ( $r$, Alice, Caddie), respectively. But one also has $s_{2} \stackrel{r}{\rightarrow} s_{2}$ since ( $r$, Bob, Caddie) is not applicable in $s_{2}$ either. And furthermore $s_{2} \stackrel{r}{\rightarrow} s_{3}$ where $s_{3}$ differs from $s_{2}$ only in that bonus $^{s_{3}}($ Alice $)=130$. This pattern repeats indefinitely.

The definition of the execution of a rule has a few equivalent formulations, and yields the following remarks. Let us consider a rule $r=(\vec{o}, \vec{T}, g, a)$, two $\mathcal{T}$-compliant states $s$ and $s^{\prime}$, and a valuation $\nu$ well-typed for $(\vec{o}: \vec{T})$ in $s$ :

- Either we have $s, \nu \models g$, and then we have $s \stackrel{r}{\rightarrow} s^{\prime}$, where $s^{\prime}$ is defined by $\left\langle s, s^{\prime}\right\rangle, \nu \models \hat{a}$; or we have $s, \nu \models \neg g$, and then we have $s \stackrel{r}{\rightarrow} s$.

- Conversely, we have $s \stackrel{r}{\rightarrow} s^{\prime}$ if there exists an instance $R$ of $r$ well-typed in $s$, that is applicable in $s$ and whose application results in $s^{\prime}$ (which can be distinct from $s$ or not). And we have $s \stackrel{r}{\rightarrow} s$ if there exists an instance $R$ of $r$ well-typed in $s$, that is not applicable in $s$.

- Since the set of instances of a type in $s$ is finite, there are finitely many well-typed object tuples for $r$ in $s$, and there is also a finite number of possible executions of $r$ in $s$.

- The only case where there is no execution of $r$ in $s$, is when there is no instance of $r$ well-typed in $s$, that is, if $\mathcal{M}_{s}^{(r)}=\emptyset$. Indeed, if an instance of $r$ well-typed in $s$ can be formed, then executing this instance gives an execution of $r$, either to a new state if the instance is applicable, or to $s$ itself otherwise.

Also, the preservation properties stated in Section 5.2.3 hold on rule instance and rule executions.

Proposition 5.3. Let $r=(\vec{o}, \vec{T}, g, a)$ be rule, $s$ be a $\mathcal{T}$-compliant state, and $\nu$ be a valuation well-typed for $(\vec{o}: \vec{T})$ in $s$. The state $s^{\prime}$ that results from the execution of the rule instance $(r, \nu)$ in $s$ is $\mathcal{T}$-compliant, and $\nu$ is well-typed for $\tau$ in $s^{\prime}$. 
Proof. If $(r, \nu)$ is applicable in $s$, then the state $s^{\prime}$ is defined by $\left\langle s, s^{\prime}\right\rangle, \nu \models \hat{a}$. By Proposition 5.2, $s^{\prime}$ is $\mathcal{T}$-compliant, and $\nu$ is well-typed for $\tau$ in $s^{\prime}$.

If $(r, \nu)$ is not applicable in $s$, then $s^{\prime}=s$. Then $s^{\prime}$ is $\mathcal{T}$-compliant and $\nu$ is well-typed for $\tau$ in $s^{\prime}$ by hypothesis.

More generally, any state that results from an execution of a rule in a $\mathcal{T}$ compliant state is $\mathcal{T}$-compliant, and a chain of executions of well-typed rule instances that starts in a $\mathcal{T}$-compliant state contains only $\mathcal{T}$-compliant states. Along this chain, the working memory and the instances of types are constant. In this context we can omit the state in the notations, and write $\mathcal{M}$ for the working memory and $\mathcal{M}(T)$ for the instances of type $T$.

\subsection{Rule Program Execution}

Building on the description of rule and rule instance execution introduced in the previous section, we define an abstract machine to describe the executions of a rule program formally. Rule engines as described in Section 5.1 can be seen as the corresponding concrete machines.

We also give a formal description of the parameters of the abstract machine identified as the selection and eligibility strategies. The next two sections present several such strategies, both intuitively and with the formalization introduced here.

As announced, we define the operational semantics (in the sense of [Plo04]) of a rule program by using transitions between configurations of an abstract machine.

\subsubsection{Configurations}

The abstract machine that we use to describe the semantics of rule programs has configurations of the form $\langle E, s\rangle$, where $s$ is a $\mathcal{T}$-compliant state and $E$ is a set of rule instances well-typed in $s$. In a given configuration, $s$ represents the current state and $E$ the set of eligible rule instances.

We describe the semantics of the execution of a rule program from a given state by defining the initial configuration of the abstract machine and the possible transitions between configurations.

\subsubsection{Initial Configuration}

As suggested by the definition of a configuration of our abstract machine, we are going to consider only the executions of a rule program that start in a $\mathcal{T}$ compliant state. Let us remind that a state is $\mathcal{T}$-compliant if each object either has a $\perp$ value for all its attributes in this state, or the set of attributes for which it has a non- $\perp$ value (the attribute span of the object) matches the definition of a type in the type system $\mathcal{T}$.

In the initial configuration for the execution of a rule program, all rule instances are eligible. This is admittedly an arbitrary choice, and the set of initially eligible rule instances could be a component of the eligibility strategy. However, it is not necessary, for the eligibility strategies that we consider, to 
include the set of initially eligible rule instances in the definition of an eligibility strategy.

We note $\mathrm{RI}_{s}(\mathcal{R})$ the set of all the instances of rules from the rule program $\mathcal{R}$ that are well-typed in $s$, that is,

$$
\mathrm{RI}_{s}(\mathcal{R})=\left\{(r, \vec{O}) \mid r \in \mathcal{R}, \vec{O} \in \mathcal{M}_{s}^{(r)}\right\} .
$$

With this definition, the initial configuration for the execution of a rule program $\mathcal{R}$ in a $\mathcal{T}$-compliant state $s_{0}$ is $\left\langle\mathrm{RI}_{s_{0}}(\mathcal{R}), s_{0}\right\rangle$.

\subsubsection{Transition Between Configurations}

A transition between two configurations of our abstract machine can occur by the application of a rule instance. This rule instance must be retained by the selection strategy among the instances that are both applicable and eligible. The resulting configuration is made of the state that results from the application of the rule instance, and of the set of eligible rules updated according to the eligibility strategy.

The above is expressed by the following transition rule. The details of the notations used in this rule are given just below; in short, $A_{s}$ and $E$ respectively denote the sets of applicable and eligible rule instances, $\mathcal{S}$ and $\mathcal{E}$ are the selection and eligibility strategies, and $R$ is the rule instance that is applied in the transition.

$$
\frac{R \in \mathcal{S}\left(A_{s} \cap E\right) \quad s \stackrel{R}{\longrightarrow} s^{\prime} \quad E^{\prime}=\mathcal{E}(E, \ldots)}{\langle E, s\rangle \stackrel{R}{\longrightarrow}\left\langle E^{\prime}, s^{\prime}\right\rangle}
$$

In this transition rule we note $A_{s}$ the set of all rule instances applicable in the state $s$ :

$$
A_{s} \stackrel{\text { def }}{=}\left\{R \in \mathrm{RI}_{s}(\mathcal{R}) \mid R \text { applicable in } s\right\} .
$$

The set $A_{s} \cap E$ thus denotes the conflict set, that is, the set of rule instances that are both applicable and eligible in $\langle E, s\rangle$. The selection strategy $\mathcal{S}$ is a function that computes a subset of the conflict set that we call the selection, and which is the abstract counterpart to the "head of the agenda" of the concrete rule engines described in Section 5.1. The selection is a set of rule instances - whereas the head of the agenda is unitary. With this choice we abstract away some implementation-dependent aspects of the selection strategies of rule engines that do not participate in the semantics of the rule program.

As a consequence, there can be several candidate rule instances for a transition from the current configuration; all of them will be considered, leading to several possible executions of the rule program. In each possible transition, the state $s^{\prime}$ is the one that results from the application in the initial state $s$ of the rule instance considered.

The eligibility strategy $\mathcal{E}$ is also a function that takes and returns a set of rule instances. It produces the set of eligible rule instances in the resulting configuration, out of the corresponding set in the initial configuration, and possibly additional arguments depending on each eligibility strategy.

The selection strategy and the eligibility strategy are parameters to the operational semantics of rule programs. Together, they are referred to as an execution strategy. They must be fixed in order for the transition system of our 
abstract machine to describe the execution of a rule program. In Section 5.5 we present some common selection strategies; in Section 5.6 we present some common eligibility strategies. In Section 6.1.1, we fix the execution strategy that we will use from Chapter 6 on.

\subsubsection{Executions of a Rule Program}

A transition sequence is a sequence of configurations where each configuration is obtained from the previous one by an application of the transition rule. Such a sequence can be finite or infinite. A transition sequence that cannot be extended is called maximal.

Definition 5.12. An execution of a rule program $\mathcal{R}$ from a $\mathcal{T}$-compliant state $s_{0}$ under the selection strategy $\mathcal{S}$ and the eligibility strategy $\mathcal{E}$, is a maximal transition sequence by the transition rule (5.1) from the initial configuration $\left\langle\mathrm{RI}_{s_{0}}(\mathcal{R}), s_{0}\right\rangle$.

Given two states $s$ and $s^{\prime}$ and a rule program $\mathcal{R}$, we note $s \stackrel{\mathcal{R} / \mathcal{S}, \mathcal{E}}{\longrightarrow} s^{\prime}$ when there is an execution of $\mathcal{R}$ under the selection strategy $\mathcal{S}$ and the eligibility strategy $\mathcal{E}$, that starts in $s$ and results in $s^{\prime}$. When either of $\mathcal{S}$ or $\mathcal{E}$, or both, are fixed they can be omitted from the notation.

Since the execution of a rule program starts in a $\mathcal{T}$-compliant state and contains only well-typed rule instances, all states in the execution are $\mathcal{T}$-compliant, and the working memory $\mathcal{M}$, the sets of instances of each type, and the set of well-typed rule instances $\operatorname{RI}(\mathcal{R})$, are constant along the execution.

It can also be noted that the executions of a program $\mathcal{R}=\{r\}$ reduced to only one rule, are not the same as the executions of $r$ as a rule, as defined in Section 5.3.5. Indeed, considering two $\mathcal{T}$-compliant states $s$ and $s^{\prime}$, we have $s \stackrel{r}{\rightarrow} s^{\prime}$ when $s^{\prime}$ results from the execution of one instance of $r$ in $s$, whereas we have $s \stackrel{\mathcal{R}}{\rightarrow} s^{\prime}$ when $s^{\prime}$ results from the execution of all the instances of $r$ on $\mathcal{M}$. In addition, the executions of $\mathcal{R}$ will depend on the chosen execution strategy, which has no effect on the execution on $r$.

\section{Example}

Let us consider the rule program $\mathcal{R}=\left\{r_{1}, r_{2}\right\}$ :

$$
\begin{aligned}
& r_{1}(p: \text { Person }): \text { p.age }<21 \rightarrow \text { p.score }:=\text { p.score }+10 \\
& r_{2}(p: \text { Person }): \text { p.score }>35 \rightarrow \text { p.score }:=\text { p.score } / 2
\end{aligned}
$$

and the following initial configuration:

$$
\begin{aligned}
\mathcal{M}_{s_{0}} & =\mathcal{M}_{s_{0}}(\text { Person })=\{\text { Alice }, \text { Bob }\} \\
s_{0} & =\text { age }^{s_{0}}: \text { Alice } \mapsto 10 \quad \text { score }^{s_{0}}: \text { Alice } \mapsto 20 \\
\text { Bob } & \mapsto 30 \quad \text { Bob } \mapsto 40 \\
E_{0} & =\left\{\left(r_{1}, \text { Alice }\right),\left(r_{1}, \text { Bob }\right),\left(r_{2}, \text { Alice }\right),\left(r_{2}, \text { Bob }\right)\right\} .
\end{aligned}
$$

Let us define a fictive execution strategy made of the selection strategy $\mathcal{S}_{\text {fic }}$ 
and the eligibility strategy $\mathcal{E}_{\text {fic }}$ defined below:

$$
\begin{aligned}
\mathcal{S}_{\text {fic }}(C) & = \begin{cases}C \cap\left\{\text { instances of } r_{1}\right\} & \text { if non empty } \\
C \cap\left\{\text { instances of } r_{2}\right\} & \text { otherwise }\end{cases} \\
\mathcal{E}_{\text {fic }}(E, k) & =\left\{\text { instances of } r_{i} \mid i>k / 2\right\} \quad \text { after } k \text { transitions. }
\end{aligned}
$$

In the initial configuration $\left\langle E_{0}, s_{0}\right\rangle$ the applicable rule instances are

$$
A_{s_{0}}=\left\{\left(r_{1}, \text { Alice }\right),\left(r_{2}, \text { Bob }\right)\right\}
$$

since $a g e^{s_{0}}($ Alice $)=10<21$ and $\operatorname{score}^{s_{0}}(\mathrm{Bob})=40>35$, but $a g e^{s_{0}}(\mathrm{Bob})=$ $30 \geq 21$ and $\operatorname{score}^{s_{0}}($ Alice $)=20 \leq 35$. As all rule instances are eligible in the initial configuration, the conflict set is equal to $A_{s_{0}}$.

The application of the selection strategy $\mathcal{S}_{\text {fic }}$ to $A_{s_{0}}$ retains only the instances of $r_{1}$, leaving only $\left(r_{1}\right.$, Alice) in the selection. The application of this rule instance causes a transition to $\left\langle E_{1}, s_{1}\right\rangle$ with

$$
\begin{array}{rlr}
s_{1} & =\text { age }^{s_{1}}: \text { Alice } \mapsto 10 \quad \text { score }^{s_{1}}: \text { Alice } \mapsto \mathbf{3 0} \\
& \text { Bob } \mapsto 30 \quad \text { Bob } \mapsto 40 \\
E_{1}= & \left\{\text { instances of } r_{i} \mid i>1 / 2\right\} \\
= & \left\{\left(r_{1}, \text { Alice }\right),\left(r_{1}, \text { Bob }\right),\left(r_{2}, \text { Alice }\right),\left(r_{2}, \text { Bob }\right)\right\} .
\end{array}
$$

The applicable rule instances in $s_{1}$ are $A_{s_{1}}=\left\{\left(r_{1}\right.\right.$, Alice $),\left(r_{2}\right.$, Bob $\left.)\right\}$ again, which leads to the same conflict set, and again only $\left(r_{1}\right.$, Alice) in the selection. The application of this rule instance causes a transition to $\left\langle E_{2}, s_{2}\right\rangle$ with

$$
\begin{aligned}
s_{2} & =\ldots \quad \text { score }^{s_{2}}: \text { Alice } \mapsto \mathbf{4 0} \quad \ldots \\
E_{2} & =\left\{\text { instances of } r_{i} \mid i>1\right\}=\left\{\left(r_{2}, \text { Alice }\right),\left(r_{2}, \text { Bob }\right)\right\} .
\end{aligned}
$$

The applicable rule instances are $A_{s_{2}}=\left\{\left(r_{1}\right.\right.$, Alice $),\left(r_{2}\right.$, Alice $),\left(r_{2}\right.$, Bob $\left.)\right\}$ in this configuration, since the scores of both Alice and Bob are greater than 35 . The conflict set is therefore $A_{s_{2}} \cap E_{2}=\left\{\left(r_{2}\right.\right.$, Alice $\left.),\left(r_{2}, \mathrm{Bob}\right)\right\}$, and is returned untouched as the selection by the selection strategy.

We have here a selection that contains two elements, and hence two possible transitions from $\left\langle E_{2}, s_{2}\right\rangle$, by either of these rule instances. Let us note $\left\langle E_{3}^{\text {Alice }}, s_{3}^{\text {Alice }}\right\rangle$ and $\left\langle E_{3}^{\text {Bob }}, s_{3}^{\text {Bob }}\right\rangle$ the configurations that result from the transition by $\left(r_{2}\right.$, Alice $)$ and by $\left(r_{2}\right.$, Bob $)$, respectively. For any $P \in\{$ Alice, Bob $\}$ we have

$$
\begin{aligned}
s_{3}^{P} & =\ldots \quad \text { score }^{s_{3}^{P}}: P \mapsto \mathbf{2 0} \quad \ldots \\
E_{3}^{P} & =\left\{\text { instances of } r_{i} \mid i>3 / 2\right\}=\left\{\left(r_{2}, \text { Alice }\right),\left(r_{2}, \text { Bob }\right)\right\} .
\end{aligned}
$$

Let us note $P^{\prime}$ the other person than $P$. In either configuration $\left\langle E_{3}^{P}, s_{3}^{P}\right\rangle$, the only applicable and eligible rule instance is $\left(r_{2}, P^{\prime}\right)$, and it is retained by $\mathcal{S}_{\text {fic }}$. From either configuration the transition by this rule instance leads to $\left\langle E_{4}, s_{4}\right\rangle$, with

$$
\begin{aligned}
& s_{4}=a g e^{s_{4}}: \text { Alice } \mapsto 10 \quad \text { score }^{s_{4}}: \text { Alice } \mapsto 20 \\
& \text { Bob } \mapsto 30 \quad \text { Bob } \mapsto 20 \\
& E_{4}=\left\{\text { instances of } r_{i} \mid i>2\right\}=\{\} \text {. }
\end{aligned}
$$


In this configuration the applicable rule instances are reduced to $A_{s_{4}}=$ $\left\{\left(r_{1}\right.\right.$, Alice $\left.)\right\}$, but this rule instance is not eligible since none is. The conflict set and hence the selection are empty. Both executions stop, with $s_{4}$ as their final state.

As a summary, we have $s_{0} \stackrel{\mathcal{R} / \mathcal{S}_{\text {fic }}, \mathcal{E}_{\text {fic }}}{\longrightarrow} s_{4}$ as the rule program $\mathcal{R}$ used in this example admits two executions under the fictive execution strategy defined for this example. These two executions start in the state $s_{0}$ and result in the state $s_{4}$. They are:

$$
\begin{array}{cccc}
\left(r_{1}, \text { Alice }\right) & \left(r_{1}, \text { Alice }\right) & \left(r_{2}, \text { Alice }\right) & \left(r_{2}, \text { Bob }\right) \\
\left(r_{1}, \text { Alice }\right) & \left(r_{1}, \text { Alice }\right) & \left(r_{2}, \text { Bob }\right) & \left(r_{2}, \text { Alice }\right) .
\end{array}
$$

Note also that the same rule instance was applied twice in the transitions $\left\langle E_{0}, s_{0}\right\rangle \rightarrow\left\langle E_{1}, s_{1}\right\rangle$ and $\left\langle E_{1}, s_{1}\right\rangle \rightarrow\left\langle E_{2}, s_{2}\right\rangle$; this happened because the rule instance was applicable in both $s_{0}$ and $s_{1}$, and the eligibility strategy did not remove it from the set of eligible rule instances until $E_{2}$. One of the major roles of the eligibility strategy is to avoid trivial loops in rule program execution, with the meaning of "trivial" varying from one strategy to the other. Some strategies even ensure that all executions of a rule program terminate.

\subsection{Selection Strategies}

One of the parameters of the abstract machine introduced in the previous section, the selection strategy determines which rule instance to apply when several are both eligible and applicable. The basic mechanism of selection strategies consists in ordering the candidate rule instances and picking the/a maximal one. This section presents the most common orders.

When several rule instances are both applicable and eligible in a given state, the choice of which to apply to perform the transition to the next configuration is the role of the selection strategy. Since the set of applicable and eligible rule instances is called the conflict set, the selection strategy is also named conflict set resolution strategy.

The selection strategies used in practice by rule engines are deterministic: they ensure that, when applied in a given state to a given conflict set, they will always return the same rule instance. This behavior is of course required for real executions of rule programs to be reproducible. However, to ensure this, rule engines need to resort to criteria such as the name of the rule or its position in a program source file, which have no actual meaning in the logic of the program, and might even be out of the control of the rule author.

This is why verification systems for rule programs use abstracted selection strategies, which ignore these additional practical criteria, and which consider that the outcome of the selection strategy is a set of rule instances. This can lead a rule program to have several executions on the abstract machine. All these executions can have the same termination status, and if terminating, can lead to the same final state. Or not.

With this choice of using abstracted selection strategies, verification systems for rule programs might report errors (non-confluences) that would actually not occur in practice, but that reflect a flaw in the rule program design, because its execution depends on internal, technical choices of the rule engine. This feature 
is analogous to the choice of interpreting numbers in the mathematical sense, versus using machine arithmetics.

In our formalization, a selection strategy is thus a function that takes a set of rule instances (the conflict set) and returns a subset of this set (the selection).

In most cases, selection strategies define an order on rule instances, and return the maximal elements of the conflict set according to this order. When the order is not strict, the returned selection can contain several elements. A selection strategy might also be defined by the lexicographic combination of various orders.

The simplest selection strategy performs no filtering of the conflict set. We call it the identity strategy. In this strategy we have

$$
\mathcal{S}_{\text {id }}(C)=C \text {. }
$$

Here are various orders that are commonly used to define selection strategies [BFKM85, Met91].

- Priority on rule instances. For this order, a rule $r=(\vec{o}, \vec{T}, g, a)$ is additionally equipped with a numerical expression $\pi_{r} \in \mathcal{E}\left(\left\{o_{1}, \ldots, o_{m}\right\}\right)$ well-typed for $(\vec{o}: \vec{T})$. The order is based on the value of this expression for each rule instance in the current state.

- Priority on rules. This order is a simplified version of the previous one, where the expressions $\pi_{r}$ are numerical constants. The priority of all instances of a rule $r$ are then equal to the number $\pi_{r}$, independently of the state.

- Strict ordering of the rules. A strict order is defined on the rules, for example by setting the rule priorities to a permutation of $\{1, \ldots, n\}$.

- Specificity of the rules. A rule $r_{1}$ is said to be more specific than another rule $r_{2}$ when one has $g_{1} \Rightarrow g_{2}$, where $g_{i}$ is the guard of $r_{i}$. This defines a partial order on rules. Some rule engines approximate this order by using empirical indications of the rule specificity, such as the number of elementary Boolean expressions in the rule guard, or the arity of the rule.

- Recency. This order is based on a numerical constant associated with each object in the working memory, called the object recency, with the idea that objects have been inserted into the working memory in some order. The recency of a rule instance is given by the maximal recency of the objects in the rule instance.

\subsection{Eligibility Strategies}

The eligibility strategy is the other parameter of the abstract machine introduced in Section 5.4. It has a more dramatic impact on execution, as it mainly influences its termination. This section presents four eligibility strategies, including the well-known refraction strategy.

Classical production rule systems such as OPS5 do not consider selection and eligibility as separate strategies [BFKM85, Met91, BDS12]. Instead, their "conflict set resolution strategies" are a composition of several selection strategies 
listed in the previous section, such as specificity or recency, and usually also include refraction, although the latter has an eliminating effect whereas the former have a selecting effect. We choose to distinguish the selection strategy from the eligibility strategy in our abstract machine, both because the two strategies are often independent, and so that we can more easily consider alternatives to refraction.

The notion of rule instance eligibility has the objective of avoiding trivial loops as illustrated on page 47 . The role of the eligibility strategy is to update the set of eligible rules, which is part of the configuration of the abstract machine.

We define the eligibility strategy as a function that returns the set of eligible rule instances for the configuration that results from the transition. The arguments to the eligibility strategy as a function vary with the definition of each strategy. However most strategies can be defined with the set of eligible rules before the transition, the rule instance that caused the transition, the initial and final states of the transition, or the initial state of the program execution.

Another representation of an eligibility strategy, proposed by Ahmed Bouajjani [Bou08], uses an automaton to describe the evolution of a rule instance during an execution of the rule program. The accepting states of this automaton correspond to configurations of the abstract machine in which the rule instance is eligible. The transitions of the automaton depend on similar criteria as the possible arguments to the eligibility strategy seen as a function, such as the rule instance that caused the abstract machine transition or the final state of this transition. Each of the eligibility strategies described further below in this section is also depicted with such an automaton.

As for selection strategies, several eligibility strategies exist in rule engine implementations. We formalize them below. What makes the difference between eligibility strategies is how drastic they are in declaring a rule instance ineligible. Some strategies make rule instances ineligible only if they have been applied, while others may make rule instances ineligible even if they have not been applied; some strategies never make rule instances eligible again once they have been made ineligible, while others allow rule instances to be included again in the set of eligible ones.

The effect of these variations is on the termination of rule program executions. If the strategy allows rule instances to become eligible again, it makes it possible to have infinite executions. Conversely, an eligibility strategy where the set of eligible rules strictly decreases on each transition will ensure the termination of all executions. On the other hand, a strategy that is very drastic in making rule instances ineligible prevents rule programs from implementing evolved reasonings based on inference and interactions between rules.

\subsubsection{Identity Eligibility Strategy}

The simplest eligibility strategy imposes no filter on the rule instances: in any configuration, all rule instances that can be formed from the rules in the rule program and objects in the working memory, are eligible. We call it the identity strategy. It can be defined equivalent by

$$
\mathcal{E}_{\text {id }}(E)=\operatorname{RI}(\mathcal{R}) \quad \text { or } \quad \mathcal{E}_{\text {id }}(E)=E .
$$


This eligibility strategy will almost always lead to infinite executions, including trivial ones like infinite application of always the same rule instance. Exceptions will occur when the action of a rule invalidates its guard, as well as variants involving cross-invalidation between rules. For this reason it is not used in practice, and its principal interest is illustrative.

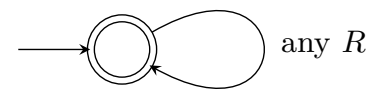

This automaton describes the eligibility of a rule instance $(r, \nu)$ and its evolution upon transitions of the abstract machine by a rule instance $R$.

Under the identity eligibility strategy, a rule instance is initially eligible and remains eligible during the whole rule program execution, no matter which rule instances are applied. This gives an automaton with only one state. This state is the initial state and is accepting. A transition of the abstract machine by any rule instance leaves the automaton in this state.

Figure 5.3: Identity Eligibility Strategy.

\subsubsection{Refraction Eligibility Strategy}

The refraction eligibility strategy is the one at work in the Rete algorithm [For82, FL92, CKMM04, dSMHP10] used in most major rule engine implementations. In the traditional presentation of this algorithm [For81, BDS12], refraction is just one step of the algorithm, whereas we describe it as an eligibility strategy, and we would gather the other steps as the selection strategy.

The refraction eligibility strategy includes cases where rule instances become eligible again after having been made ineligible. As a consequence, this strategy does not guarantee termination of executions. On the other hand, it makes it possible to implement complex inference-based algorithms with rule programs.

It can be noted that this ability has been of lesser use recently, as rule programs tend to be used more and more to implement algorithms that are less and less stateful. Although the Rete algorithm can still properly execute these less stateful rule programs, other execution strategies such as the sequential one have been introduced, targeting these rule programs with more efficient implementations. The sequential execution strategy is described in Section 5.6.3; a comparison between this strategy and refraction is given in [BDS12].

As mentioned in the RIF-PRD recommendation [dSMHP10]: "The essential idea of refraction is that a given instance of a rule must not be fired more than once as long as the reasons that made it eligible for firing hold." Refraction thus states that, once executed, a rule instance cannot be re-executed before it becomes inapplicable, and then of course applicable again. Let us illustrate this statement with the example below, before transposing it into an eligibility strategy.

Let us illustrate this statement with the example below, before transposing it into an eligibility strategy. 
Example. Consider the rule program $\mathcal{R}=\left\{r_{1}, r_{2}\right\}$ :

$$
\begin{aligned}
& r_{1}(p: \text { Person }): \text { p.age }<21 \rightarrow \text { p.score }:=\text { p.score }+10 \\
& r_{2}(p: \text { Person }): \text { p.score }>25 \rightarrow \text { p.score }:=\text { p.score } / 2
\end{aligned}
$$

and the following initial configuration:

$$
\begin{aligned}
\mathcal{M}_{s_{0}} & =\mathcal{M}_{s_{0}}(\text { Person })=\{\text { Alice }\} \\
s_{0} & =a^{s_{0}}: \text { Alice } \mapsto 10 \quad \text { score }^{s_{0}}: \text { Alice } \mapsto 40 \\
E_{0} & =\left\{\left(r_{1}, \text { Alice }\right),\left(r_{2}, \text { Alice }\right)\right\} .
\end{aligned}
$$

In this initial state, both rule instances $\left(r_{1}\right.$, Alice) and $\left(r_{2}\right.$, Alice) are eligible and applicable. Consider the execution where $\left(r_{2}\right.$, Alice) gets executed. This sets Alice's score to 20, which invalidates the guard of $r_{2}$ on Alice. The only applicable rule instance $\left(r_{1}\right.$, Alice) is then executed, causing Alice's score to be set to 30 . The refraction strategy states that $\left(r_{2}\right.$, Alice) can then be executed again.

The refraction eligibility strategy states that a rule instance becomes ineligible when executed, and becomes eligible again as soon as it becomes inapplicable.

The strategy is thus defined as follows, in a transition $\langle E, s\rangle \stackrel{R}{\longrightarrow}\left\langle E^{\prime}, s^{\prime}\right\rangle:$

$$
\mathcal{E}_{\text {refraction }}\left(E, R, s^{\prime}\right)=E \backslash\{R\} \cup\left\{R^{\prime} \in \mathrm{RI}(\mathcal{R}) \mid R^{\prime} \text { is not applicable in } s^{\prime}\right\} \text {. }
$$

The refraction eligibility strategy underlines the difference between the applicability and the eligibility of a rule instance. The definition of $\mathcal{E}_{\text {refraction }}$ above states that all inapplicable rule instances are eligible after each transition. Actually this adds to the set of already eligible rule instances only the ones that satisfy the two following conditions:

(i) The rule instance has been applied at least once in this rule program execution, as the rule instances that have never been applied have remained eligible since the initial configuration of the rule program execution.

(ii) The rule instance was applicable up until the initial configuration of the transition, otherwise it would have been added during a previous transition.

In other words, the rule instances added are exactly the ones that have been already applied in this execution, and that have been made inapplicable by the last transition. Of course, these newly eligible rule instances will be in the conflict set only when their guards are valid again, but that is not the role of the eligibility strategy and, as far as eligibility is concerned, the guard turning invalid is sufficient.

This mechanism ensures that once executed, a rule instance will not be executed a second time before it becomes first inapplicable and then applicable again, as stated by the Rete algorithm.

Example. Consider the same rule program and configuration as in the previous example. The initial set of eligible rules is

$$
E_{0}=\operatorname{RI}(\mathcal{R})=\left\{\left(r_{1}, \text { Alice }\right),\left(r_{2}, \text { Alice }\right)\right\}
$$




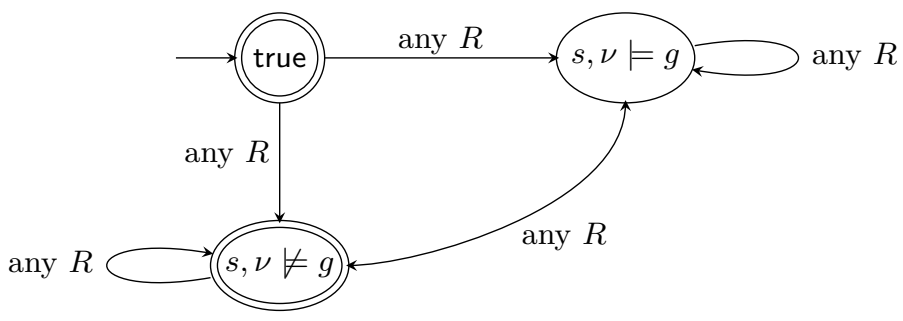

This automaton describes the eligibility of a rule instance and its evolution upon transitions of the abstract machine. Each state of the automaton is annotated with a condition (expressed on the state of the abstract machine) that is required to hold for a transition to this state to be possible.

Under the refraction eligibility strategy, a rule instance $(r, \nu)$ with $r=$ $(\vec{o}, \vec{T}, g, a)$ is initially eligible and subsequently becomes or remains eligible when a rule instance $R$ (possibly $(r, \nu)$ itself) is applied and brings the abstract machine into a state $s$ where the rule instance $(r, \nu)$ is applicable, that is, such that $s, \nu \models g$. This gives an automaton with an accepting initial state, and two other states corresponding to the cases where the rule instance considered is applicable or not. The state where the rule instance is not applicable is accepting.

Figure 5.4: Refraction Eligibility Strategy.

We chose to consider the execution of $\left(r_{2}\right.$, Alice), which invalidates its own guard; the resulting configuration is therefore

$$
\begin{aligned}
s_{1} & =\text { age }^{s_{1}}: \text { Alice } \mapsto 10 \quad \text { score }^{s_{1}}: \text { Alice } \mapsto 20 \\
E_{1} & =E_{0} \backslash\left\{\left(r_{2}, \text { Alice }\right)\right\} \cup\left\{\left(r_{2}, \text { Alice }\right)\right\}=\left\{\left(r_{1}, \text { Alice }\right),\left(r_{2}, \text { Alice }\right)\right\} .
\end{aligned}
$$

In this transition, the rule instance $\left(r_{2}\right.$, Alice) has been removed from the set of eligible ones because it was applied in the transition, and then added back as a rule instance that was made inapplicable by the transition. The fact that it was made inapplicable by its own application is coincidental.

In the configuration $\left\langle E_{1}, s_{1}\right\rangle$ the only applicable rule instance is $\left(r_{1}\right.$, Alice). As it is eligible it gets applied, causing the transition to $\left\langle E_{2}, s_{2}\right\rangle$, with

$$
\begin{aligned}
& s_{2}=\text { age }^{s_{2}}: \text { Alice } \mapsto 10 \quad \text { score }^{s_{2}}: \text { Alice } \mapsto 30 \\
& E_{2}=E_{1} \backslash\left\{\left(r_{1}, \text { Alice }\right)\right\} \cup\{\}=\left\{\left(r_{2}, \text { Alice }\right)\right\}
\end{aligned}
$$

Here, both rule instances are applicable but only $\left(r_{2}\right.$, Alice $)$ is eligible. As above, when this rule instance is applied, it invalidates its own guard, which makes it eligible again. The configuration $\left\langle E_{3}, s_{3}\right\rangle$ is therefore

$$
\begin{aligned}
s_{3} & =\text { age }^{s_{3}}: \text { Alice } \mapsto 10 \quad \text { score }^{s_{3}}: \text { Alice } \mapsto 15 \\
E_{3} & =E_{2} \backslash\left\{\left(r_{2}, \text { Alice }\right)\right\} \cup\left\{\left(r_{2}, \text { Alice }\right)\right\}=\left\{\left(r_{2}, \text { Alice }\right)\right\}
\end{aligned}
$$

In this configuration, the rule instance $\left(r_{1}\right.$, Alice) is applicable but not eligible, and $\left(r_{2}\right.$, Alice) is eligible but not applicable. This execution of the rule program thus terminates with the state $s_{3}$ as its final state. 
It is interesting to note that if Alice's score in the initial state $s_{0}$ is 60 , then the application of $\left(r_{2}\right.$, Alice) sets it to 30 . With this value, the rule instance is still applicable, and hence it is not added back to the set $E_{1}$ of eligible rule instances. This execution ends with no eligible rule instances after the application of ( $r_{1}$, Alice), because it sets Alice's score to 40 without invalidating any guard.

\subsubsection{Sequential Execution Strategy}

The sequential execution strategy has recently emerged [IBM11, Red10, Fai07] as a competitive option for rule engine implementations. This occurred as stateful rule programs became less frequent than stateless ones, as the latter can be compiled more efficiently with this execution strategy than with the Rete algorithm.

Neither the identity nor the refraction eligibility strategies just described, nor the one-shot strategy described further below, is tied to a particular selection strategy. In contrast, the sequential eligibility strategy comes with a companion selection strategy, forming a complete execution strategy.

The sequential execution strategy states that the rules and objects are to be taken into consideration in some sequential order, and that each rule instance is to be applied if it is applicable at the moment it is considered. If a rule instance has been considered for application and only later becomes applicable, for example as the consequence of another rule instance application, the rule instance is not to be considered again.

To get this behavior, the roles of the selection and eligibility strategies in the sequential execution strategy will be for the eligibility strategy to define the set of rule instances under consideration, and for the selection strategy to ensure that the rule instances are picked in the proper order.

The sequential execution strategy is based on a strict ordering of the rules in the rule program, and of the objects in the working memory. Since only well-typed rule instances are formed, it suffices in fact to define a strict order within the instances of each type.

The orders on the rules and on the instances of each type lexicographically define a strict order $<$ on the rule instances. The sequential execution strategy is then defined as follows:

(i) The sequential selection strategy returns the minimal rule instance from the conflict set:

$$
\mathcal{S}_{\text {sequential }}(C)=\{R \in C \mid R \text { is minimal with respect to }<\} \text {. }
$$

(ii) The sequential eligibility strategy retains for further consideration only the rule instances that follow (are greater than) the rule instance just applied; in a transition $\langle E, s\rangle \stackrel{R}{\rightarrow}\left\langle E^{\prime}, s^{\prime}\right\rangle$ :

$$
\mathcal{E}_{\text {sequential }}(E, R)=\left\{R^{\prime} \in E \mid R<R^{\prime}\right\} \text {. }
$$

It is depicted in Fig. 5.5. 


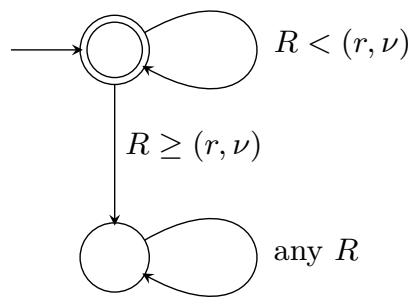

This automaton describes the eligibility of a rule instance $(r, \nu)$ and its evolution upon transitions of the abstract machine by a rule instance $R$.

Under the sequential eligibility strategy, a rule instance remains eligible as long as the rule instance that is applied is strictly smaller with respect to the order defined on rule instances. Once the rule instance, or a greater one, is applied, it ceases to be eligible.

Figure 5.5: Sequential Eligibility Strategy.

\subsubsection{One-Shot Eligibility Strategy}

The one-shot eligibility strategy states that, in each transition of the rule program execution, the last rule instance applied is removed from the set of eligible ones. It is thus defined as follows, in a transition $\langle E, s\rangle \stackrel{R}{\longrightarrow}\left\langle E^{\prime}, s^{\prime}\right\rangle$ :

$$
\mathcal{E}_{\text {oneshot }}(E, R)=E \backslash\{R\} \text {. }
$$

According to this strategy, each rule instance is eligible until it gets executed. The fact that the set of eligible rules decreases upon each transition ensures that all executions of the rule program under this strategy are finite. In the final state, the rule instances that might still be eligible are inapplicable.

The one-shot eligibility strategy is depicted in Fig. 5.6.

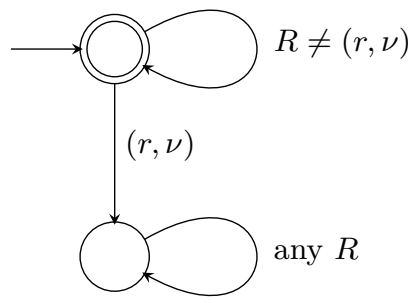

This automaton describes the eligibility of a rule instance $(r, \nu)$ and its evolution upon transitions of the abstract machine by a rule instance $R$.

Under the one-shot eligibility strategy, a rule instance remains eligible until it is applied. The application of other rule instances has no effect on its eligibility.

Figure 5.6: One-Shot Eligibility Strategy.

The one-shot eligibility strategy can be seen as a trade-off between the refraction and sequential eligibility strategies. Like the sequential strategy, it never adds rule instances to the set of eligible ones. Like the refraction strategy, 
it makes rule instances ineligible only when they are applied. This combination ensures the termination of all rule program executions, while not imposing any precedence between the rules or their instances. As a result, any rule instance has the opportunity of being applied after it gets applicable, but only once since its application makes it ineligible.

The one-shot eligibility strategy is equivalent to the refraction strategy when no rule instance appears in the conflict set after it has been executed once. This will happen if there is no way for a rule instance to become inapplicable, and then applicable again, as the result of the application of one or several rule instances. Such a condition is quite complex to express in general, however it admits sufficient conditions at the syntactic level. Consider for example a program where the set of attribute symbols that occur in the guards is disjoint from the set of attribute symbols that are updated in the rule actions. This program will have the same executions under the refraction and the one-shot eligibility strategies. Such rule programs exist, and are sometimes referred to as filtering programs. 
70 CHAPTER 5. OPERATIONAL SEMANTICS OF RULE PROGRAMS 
Part II

\section{A Hoare Logic for Rule Programs}





\section{Chapter 6}

\section{Correctness of Rule Programs}

We have just defined the operational semantics of a rule program by the transitions it makes possible between configurations of an abstract machine. We have seen that this establishes a relation between the initial and final states of the possible executions of the rule program.

We now study the correctness of a rule program from the viewpoint of this relation. In other words, requirements on the correctness of a rule program are expressed through assertions on the initial and final states in a rule program execution. For this purpose, we use correctness formulas of the well-known form $\{p\} S\{q\}$.

In this chapter, we define and discuss correctness formulas for rules, and then for rule programs. Chapters 8 to 10 introduce proof rules to reason on such correctness formulas.

\subsection{Preliminaries}

In this chapter and in the subsequent ones, we use the one-shot execution strategy, the definition of which is reminded here. As its name suggests, the one-shot execution strategy forbids a rule instance to be executed twice. A consequence is that all rule programs terminate when executed under this strategy. In the previous chapter we defined the general transition rule for the abstract machine that describes executions of a rule program. Here we specialize this transition rule for the one-shot execution strategy.

This section also briefly reminds standard concepts such as correctness formulas, proofs, or proof systems.

\subsubsection{Fixing the Execution Strategy}

From now on, and unless otherwise noted, the rule program executions that we are considering use the identity selection strategy and the one-shot eligibility strategy, collectively referred to as the one-shot execution strategy. Let us remind their definitions: 
(i) The identity selection strategy defines the selection as equal to the conflict set, leaving unspecified the choice of the rule instance to be applied:

$$
\mathcal{S}_{\text {id }}(C)=C \text {. }
$$

(ii) The one-shot eligibility strategy makes the last applied rule instance ineligible; that is, in a transition $\langle E, s\rangle \stackrel{R}{\longrightarrow}\left\langle E^{\prime}, s^{\prime}\right\rangle$ :

$$
\mathcal{E}_{\text {oneshot }}(E, R)=E \backslash\{R\} \text {. }
$$

The selection and eligibility strategies have a central role in the transition rule (5.1) of the abstract machine that describes the execution of rule programs. The use of the identity selection strategy and one-shot eligibility strategy in this transition rule yields the specialized transition rule below:

$$
\frac{R \in A_{s} \quad R \in E \quad s \stackrel{R}{\rightarrow} s^{\prime} \quad E^{\prime}=E \backslash\{R\}}{\langle E, s\rangle \stackrel{R}{\longrightarrow}\left\langle E^{\prime}, s^{\prime}\right\rangle} .
$$

Given two $\mathcal{T}$-compliant states $s$ and $s^{\prime}$, we will from now on note $s \stackrel{\mathcal{R}}{\longrightarrow} s^{\prime}$ when there exists a maximal transition sequence by the transition rule (6.1) from the configuration $\left\langle\mathrm{RI}_{s}(\mathcal{R}), s\right\rangle$ to a configuration $\left\langle E^{\prime}, s^{\prime}\right\rangle$.

As mentioned before, the one-shot eligibility strategy ensures the termination of all executions of all rule programs. In the final configuration $\left\langle E^{\prime}, s^{\prime}\right\rangle$ of an execution of a rule program $\mathcal{R}$, for any instance $R=(r, \nu)$ of a rule $r=(\vec{o}, \vec{T}, g, a)$ of $\mathcal{R}$, one has either $R \notin E^{\prime}$ or $s^{\prime}, \nu \models \neg g$. Conversely, in a configuration $\left\langle E^{\prime}, s^{\prime}\right\rangle$ that satisfies the latter, no rule of $\mathcal{R}$ can be executed.

\subsubsection{Correctness Formulas, Proofs, Proof Systems}

Correctness formulas of the form $\{p\} S\{q\}$ have been introduced by Hoare [Hoa69], as an axiomatic formulation of Floyd's method for proving partial correctness [Flo67]. The central element $S$ in such a correctness formula is a statement or a program; the assertions $p$ and $q$ express conditions on the initial and final states of executions of $S$, and hence act as pre- and postconditions of $S$.

This formalism is widely used [dBdBZ80, Apt81, Apt84, Old84] to describe the correctness of many kinds of programs, from their input-output standpoint. We contribute with a definition of correctness formulas of this form for rules in the next section, and for rule programs in Section 6.4.

Proofs are built by deriving new correctness formulas out of already known correctness formulas, by means of proof rules that are gathered in proof systems. We place ourselves in the context of the standard definitions of proof rules, proof systems, and of proofs built using them [AdBO09, pp. 38-39]. A proof rule is presented as:

$$
\begin{array}{lll}
\varphi_{1} & \cdots & \varphi_{n} \\
\varphi & \text { where } \ldots
\end{array}
$$

Such a proof rule means that if the formulas $\varphi_{1}, \ldots, \varphi_{n}$ above the horizontal line (called the premises of the rule) hold, then the formula $\varphi$ below the line (called the conclusion of the rule) holds. An optional side condition may restrict 
the cases where the rule can be applied. A proof rule with no horizontal line is called an axiom scheme, and can be read as a proof rule with true premises.

The (CONSEQUENCE) proof rule below is used, sometimes implicitly, to exploit logical implications between assertions in order to generalize a correctness formula. The proof rule is given, for example, in [Apt81], where the combination of assertions and correctness formulas in proof rules is also discussed.

$$
\frac{p \rightarrow p^{\prime} \quad\left\{p^{\prime}\right\} S\left\{q^{\prime}\right\} \quad q^{\prime} \rightarrow q}{\{p\} S\{q\}}
$$

(CONSEQUENCE)

\subsection{Correctness Formula for a Single Rule}

This section defines correctness formulas of the form $\{p\} r\{q\}$ where $r$ is a rule. The pre- and postconditions are formulas with free variables, which may be included in the rule variables, or not (as will be the case in Part III to express interference freedom conditions).

The meaning of $\{p\} r\{q\}$ follows the standards, that is, any execution of $r$ started in a state that satisfies $p$ ends in a state that satisfies $q$.

\section{Syntax}

Definition 6.1. A correctness formula for a rule is a formula of the form $\{p\} r\{q\}$, where $r$ is a rule, and $p$ and $q$ are first-order, flat, possibly quantified formulas from $\mathcal{F}(\mathrm{Var})$. The formulas $p$ and $q$ are called the pre- and postcondition, respectively.

The free variables in the correctness formula $\{p\} r\{q\}$ are those found in $p$, in $r$, or in $q$. The variables found in the rule $r=(\vec{o}, \vec{T}, g, a)$ are the $o_{1}, \ldots, o_{m}$; those in $p$ and $q$ may be in this set, or not. We call focused a correctness formula where only the rule variables appear as free variables of the pre- and postconditions.

Definition 6.2. A correctness formula for a rule $\{p\} r\{q\}$ is focused on the rule $r$ if the pre- and postconditions $p$ and $q$ are assertions focused on $r$. That is, as defined in Section 4.2.3, the free variables of $p$ and $q$ are rule variables of $r$, and $p$ and $q$ are well-typed for the type constraint implied by the rule variable types.

\section{Typing}

In a rule $r=(\vec{o}, \vec{T}, g, a)$, the variables are typed by the type constraint $(\vec{o}: \vec{T})$. A type constraint on the free variables of a correctness formula for a rule $\{p\} r\{q\}$ will thus include this constraint, and may also include constraints on the additional free variables appearing in the pre- and postconditions.

Definition 6.3. A correctness formula for a rule $\{p\} r\{q\}$ is well-typed for a type constraint $\tau$ on its free variables if the formulas $p$ and $q$ are well-typed for $\tau$.

We can note that a focused correctness formula is always well-typed for any type constraint on its free variables. 


\section{Semantics}

In line with the usual semantics of correctness formulas [Hoa69, Apt81], a correctness formula for a rule means that any execution of the rule in a state that satisfies the precondition results in a state that satisfies the postcondition. There is no mention of termination here, since an execution of a single rule always terminates.

Definition 6.4. Let $\{p\} r\{q\}$ be a correctness formula for a rule, well-typed for a type constraint $\tau$ on its free variables. The correctness formula holds if

for any $\mathcal{T}$-compliant states $s$ and $s^{\prime}$, and any valuation $\nu$ well-typed for $\tau$ in $s$, such that

one has

$$
s, \nu \models p \quad \text { and } \quad s \stackrel{(r, \nu)}{\longrightarrow} s^{\prime}
$$

$$
s^{\prime}, \nu \models q .
$$

As a reminder, the notation $s \stackrel{(r, \nu)}{\longrightarrow} s^{\prime}$ means that $s^{\prime}$ is the state that results from the execution of the rule $r$ in the state $s$ under the valuation $\nu$, which is defined in Section 5.3.4 as the state that results from the execution of the action of $r$ in $s$ under $\nu$ if the rule instance $(r, \nu)$ is applicable in $s$, and as $s$ itself otherwise.

Let us also remind that a valuation $\nu$ is well-typed for a type constraint $\tau$ in a state $s$, if it maps each variable in the domain of the type constraint to an instance of its associated type, that is, for any $\xi \in \operatorname{Dom}(\tau)$ one has $\nu(\xi) \in \mathcal{M}_{s}(\tau(\xi))$. The type constraint $\tau$ mentioned by Definition 6.4 on the free variables of the correctness formula is thus key to assess the truth value of the correctness formula. Indeed, the objects used to execute the rule and to assess the pre- and postconditions of the correctness formula are the instances of the types of these variables as constrained by $\tau$. This property is visible in the examples below.

In a correctness formula focused on a rule, the free variables are all variables of the rule, and hence are typed by construction. Some correctness formulas that appear in the interference freedom conditions of Part III are not focused on a rule; however, all the free variables in these correctness formulas are still variables of rules in the program, and hence are typed by the corresponding rule definitions.

\section{Examples}

Example. Consider the rule

$$
\mathrm{r}(\mathrm{x}: \text { Person }): \mathrm{x} . \text { age } \leq 12 \rightarrow \mathrm{x} . \text { status }:=\text { Child }
$$

and the following correctness formula for $r$

$$
\{x . \text { age }=10\} r\{\neg(\text { x.status }=\text { Adult })\} .
$$

Consider now a $\mathcal{T}$-compliant state $s$ and a valuation $\nu$ well-typed for ( $x$ :Person), such that the precondition of the correctness formula holds in $s$ under $\nu$, that is, $s, \nu \models x$.age $=10$. We thus have age $e^{s}(\nu(x))=10$, hence $a g e^{s}(\nu(x)) \leq 12$, that is, the guard of the rule $r$ holds in $s$ under $\nu$. 
As a consequence, if $s^{\prime}$ is the state that results from the execution of $r$ in $s$ under $\nu$, it is defined by $\left\langle s, s^{\prime}\right\rangle, \nu \models \hat{a}$ with $a \equiv x$.status $:=$ Child, that is,

$$
\begin{gathered}
\left\langle s, s^{\prime}\right\rangle, \nu \models x . \text { status }^{\prime}=\operatorname{Child} \wedge \forall o\left(o \neq x \rightarrow \text { o.status }^{\prime}=\text { o.status }\right) \\
\wedge \bigwedge_{f \in \operatorname{Attr} \backslash\{\text { status }\}} \forall o\left(\text { o.f } f^{\prime}=o . f\right) .
\end{gathered}
$$

In particular, we have status $s^{\prime}(\nu(x))=$ Child, and hence $s^{\prime}, \nu \models \neg(x$.status $=$ Adult).

The postcondition holds in $s^{\prime}$ under $\nu$, meaning that the correctness formula for $r$ holds.

Example. Consider the same rule as above, and the following correctness formula for $r$.

$$
\{x . \text { age }>10\} r\{x . \text { status }=\text { Child }\} .
$$

This correctness formula does not hold. Indeed, consider an instance Alice of the type Person, a valuation $\nu$ such that $\nu(x)=$ Alice, and a $\mathcal{T}$-compliant state $s$ such that $a g e^{s}($ Alice $)=30$ and status $^{s}($ Alice $)=$ Adult.

In the state $s$ and under the valuation $\nu$, the precondition holds, that is, $s, \nu \models x$.age $>10$. On the other hand, the guard of the rule does not hold in $s$ under $\nu$, since $a g e^{s}$ (Alice) $=30 \not 12$. The state that results from the execution of $r$ in $s$ under $\nu$ is therefore $s$ itself, where $\operatorname{status}^{s}$ (Alice) $=$ Adult $\neq$ Child, that is, $s, \nu \not \models x$.status $=$ Child.

There is a state and a valuation where the precondition holds, but the postcondition does not hold in the state that results from the execution of the rule. The correctness formula for $r$ thus does not hold.

Example. Consider the rule used in the previous examples, and the following correctness formula, with the type constraint ( $y$ : Person):

$$
\{x . \text { age }<10 \wedge y . \text { status }=\text { Child }\} r\{\text { x.status }=y . \text { status }\} .
$$

The valuation to consider to apply Definition 6.4 must now be well-typed for $((x, y)$ : (Person, Person $))$. Consider such a valuation $\nu$ and a $\mathcal{T}$-compliant state $s$, such that the precondition of the correctness formula holds in $s$ under $\nu$, that is, age $(\nu(x))=10$ and status ${ }^{s}(\nu(y))=$ Child. Then the guard of the rule $r$ holds in $s$ under $\nu$, and the state $s^{\prime}$ that results from the execution of $r$ in $s$ under $\nu$ is defined by

$$
\begin{aligned}
\left\langle s, s^{\prime}\right\rangle, \nu \models & x . \text { status }^{\prime}=\text { Child } \wedge \forall o\left(o \neq x \rightarrow \text { o.status }^{\prime}=\text { o.status }\right) \\
& \wedge \bigwedge_{f \in \operatorname{Attr} \backslash\{\text { status }\}} \forall o\left(\text { o. } f^{\prime}=o . f\right) .
\end{aligned}
$$

In particular, we have status $s^{\prime}(\nu(x))=$ Child.

As for $y$, we should distinguish whether it is equal to $x$ under $\nu$, or not. If it is, then status $s^{\prime}(\nu(y))=$ status $^{s^{\prime}}(\nu(x))$; it it is not, then status $s^{s^{\prime}}(\nu(y))=$ $\operatorname{status}^{s}(\nu(y))$ by the second conjunct above. In all cases, status $s^{\prime}(\nu(y))=$ Child and hence $s^{\prime}, \nu \models x$.status $=y$.status. The correctness formula for $r$ holds. 
In the examples above, it can be noted that in a correctness formula $\{p\} r\{q\}$, the precondition $p$ might or might not imply the guard of the rule. Therefore, the correctness formula must be considered both in the case where the execution of the rule results in the application of its action, and in the case where it results in a skip operation. The interaction between the precondition and the guard is discussed further below, at the end of Section 6.3 .2 on page 81 .

\subsection{Rules Compared to Conditional Statements}

Before comparing rule programs with parallel or nondeterministic programs in Chapter 7 , we can notice that a rule considered alone has the same behavior as a conditional command, in the sense that they both satisfy the same correctness formulas.

It appears from the definition of the execution of a rule in Section 5.3.5 that a rule $r=(\vec{o}, \vec{T}, g, a)$ is in some sense similar to the imperative command if $g$ then $a$ else skip fi. Below we briefly introduce the statements in this command, so as to connect their standard definitions to our formal framework; then we show that a rule and the command above have the same behavior from the viewpoint of the correctness formulas they satisfy.

\subsubsection{Loop-Free Programs}

\section{Syntax}

We consider the standard statements in a loop-free imperative program [Hoa69, Man03]. In Fig. 6.1, we provide a grammar for their language $\mathcal{L}(V)$. As usual, the language is parameterized by a set of variables $V \subseteq$ Var. Although loop-free programs are more general than the conditional command mentioned above, we define them now because we will use them as processes of parallel programs in the next chapter.

The language $\mathcal{L}(V)$ includes the skip statement and the assignment defined in Section 3.4 by the $\mathcal{A}(V)$ language. It combines these statements with the sequence and conditional control structures.

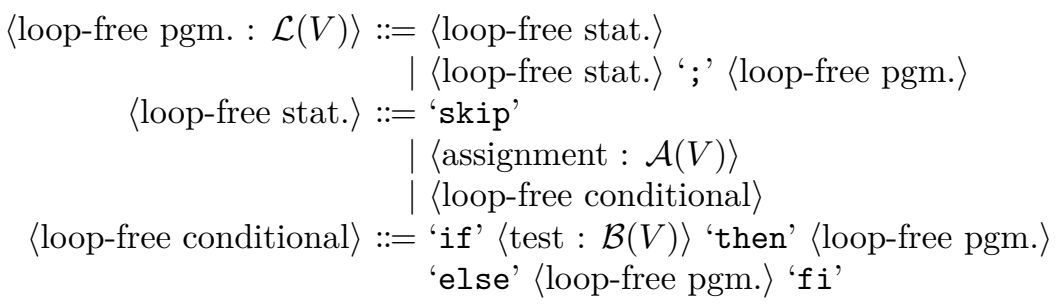

Figure 6.1: Loop-free programs.

The variables that occur in a loop-free program may come with associated types. For example, if the loop-free program is computed out of a rule $r=$ $(\vec{o}, \vec{T}, g, a)$, it will inherit its variables and their types $(\vec{o}: \vec{T})$.

We define that a loop-free program is well-typed for a type constraint by induction on its structure, based on well-typed formulas and assignment. 


\section{Semantics}

We adopt the standard semantics for loop-free programs, as defined below. We consider executions in $\mathcal{T}$-compliant states of loop-free programs that are welltyped for some given type constraint $\tau$.

We say that $s^{\prime}$ is a state that results from the execution of a program $L \in$ $\mathcal{L}($ Var $)$ in a state $s$, and we note $s \stackrel{L}{\rightarrow} s^{\prime}$, if there exists a valuation $\nu$ well-typed for $\tau$, such that the program results in $s^{\prime}$ when executed in $s$ under $\nu$. We define $s^{\prime}$ by induction on the structure on the program, as follows.

- The execution of the skip statement in $s$ under $\nu$ (actually under any valuation) results in $s$ itself.

- The execution of an assignment in $s$ under $\nu$ is defined in Section 4.4.2.

- The execution of a conditional statement if $b$ then $L_{1}$ else $L_{2}$ fi in $s$ under $\nu$, where $b \in \mathcal{B}(V)$ is a Boolean expression, and $L_{1}$ and $L_{2}$ are loop-free programs, results in the state that results from the execution of $L_{1}$ in $s$ under $\nu$ if $b$ holds in $s$ under $\nu$; and in the state that results from the execution of $L_{2}$ in $s$ under $\nu$ otherwise.

- The execution of a sequence $S ; L$ in $s$ under $\nu$, where $S$ is a loop-free statement and $L$ is a loop-free program, results in the state that results from the execution of $L$ in the state that resulted from the execution of $S$ in $s$ under $\nu$.

Example. Consider the loop-free program

$$
\mathrm{L}: \text { if } p . \text { age }>21 \text { then p.status }:=\text { Adult else p.status }:=\text { Child } \mathrm{fi}
$$

with the following type constraint, instances, and initial state:

$$
\begin{aligned}
& \tau: p \mapsto \text { Person } \\
& \mathcal{M}(\text { Person })=\{\text { Alice }, \text { Bob }\} \\
& s=\text { age }^{s}: \text { Alice } \mapsto 12 \quad \text { status }^{s}: \text { Alice } \mapsto \text { Unknown } \\
& \text { Bob } \mapsto 30 \quad \text { Bob } \mapsto \text { Unknown. }
\end{aligned}
$$

The valuations that are well-typed for $\tau$ in $s$ may map $p$ only onto Alice or onto Bob. There are therefore only two states that result from an execution of $L$ in $s$ under a valuation well-typed for $\tau$, namely $s_{1}$ and $s_{2}$ as defined below:

$$
\begin{array}{rlrl}
s_{1}=\text { age }^{s_{1}}: \text { Alice } & \mapsto 12 & \text { status }^{s_{1}}: \text { Alice } & \mapsto \text { Child } \\
\text { Bob } & \mapsto 30 & \text { Bob } & \mapsto \text { Unknown } \\
s_{2}=\text { age }^{s_{2}}: \text { Alice } & \mapsto 12 \\
\text { Bob } & \mapsto 30 & \text { status }^{s_{2}}: \text { Alice } & \mapsto \text { Unknown } \\
\text { Bob } & \mapsto \text { Adult. }
\end{array}
$$

The states that result from executions of the program $L$ in $s$ are thus $s_{1}$ and $s_{2}$. 


\section{Correctness formulas, axioms, and proof rules}

Correctness formulas $\{p\} L\{q\}$ for loop-free programs have the standard meaning, formalized as follows. Given a loop-free program $L \in \mathcal{L}($ Var $)$, two assertions $p, q \in \mathcal{F}(\mathrm{Var})$, and a type constraint $\tau$ for which $L, p$ and $q$ are well-typed, the correctness formula $\{p\} L\{q\}$ is well-typed for $\tau$, and it holds if for any $\mathcal{T}$-compliant states $s$ and $s^{\prime}$, and any valuation $\nu$ well-typed for $\tau$, such that $s, \nu \models p$ and $s \stackrel{L}{\rightarrow} s^{\prime}$, one has $s^{\prime}, \nu \models q$.

We thus inherit the standard axioms schemes and proof rules attached with skip, the assignment and the conditional statement [Hoa69, Apt81, Mor82, AdBO09]. We remind them below, with $p, q, r \in \mathcal{F}(\mathrm{Var})$ and the notations used above for the various cases of loop-free programs.

$$
\begin{gathered}
\{p\} \text { skip }\{p\} \\
\{q[f[o \mapsto e] / f]\} \text { o.f }:=e\{q\} \\
\{p \wedge b\} L_{1}\{q\} \quad\{p \wedge \neg b\} L_{2}\{q\} \\
\frac{\{p\} \text { if } b \text { then } L_{1} \text { else } L_{2} \text { fi }\{q\}}{\{p\} S\{r\} \quad\{r\} L\{q\}} \\
\frac{\{p\} S ; L\{q\}}{}
\end{gathered}
$$

\subsubsection{Rules vs. Conditional Statements}

We now formally expose the equivalence between a rule $r=(\vec{o}, \vec{T}, g, a)$ and the imperative command if $g$ then $a$ else skip fi. To this end, we first define the latter as the loop-free program derived from the former rule, then we prove that they satisfy the same correctness formulas.

Definition 6.5. Given a rule $r=(\vec{o}, \vec{T}, g, a)$, the loop-free program derived from $r$ is the program

$$
L_{r}(\vec{o}: \vec{T}): \text { if } g \text { then } a \text { else skip fi }
$$

The loop-free program derived from a rule goes with the type constraint attached to the rule. It is by construction well-typed for it.

A rule and the loop-free program derived from it have the same behavior, in the sense that they satisfy the same correctness formulas.

Proposition 6.1. Given a rule $r=(\vec{o}, \vec{T}, g, a)$ and two assertions $p, q \in \mathcal{F}$ (Var) that form a correctness formula well-typed for some type constraint $\tau$ including $(\vec{o}: \vec{T})$, the correctness formula $\{p\} r\{q\}$ for the rule $r$ holds if and only if the correctness formula $\{p\} L_{r}\{q\}$ for the loop-free program derived from $r$ holds.

Proof. We have $L_{r} \equiv$ if $g$ then $a$ else skip fi. Let $s$ be a $\mathcal{T}$-compliant state and $\nu$ be a valuation well-typed for $\tau$ in $s$, such that $s, \nu \models p$.

Case 1: $s, \nu \models g$. Then the state $s^{\prime}$ that results from the execution of the rule instance $(r, \nu)$ in $s$ is defined in Section 5.3 .4 by $\left\langle s, s^{\prime}\right\rangle, \nu \models \hat{a}$. On the other hand, the state that results from the execution of $L_{r}$ in $s$ under $\nu$ is defined as the state that results from the execution of $a$ in $s$ under $\nu$, that is, $s^{\prime}$ as well.

Case 2: $s, \nu \not \forall g$. Then the state $s^{\prime}$ that results from the execution of the rule instance $(r, \nu)$ in $s$ is defined to be $s$ itself. On the other hand, the state that 
results from the execution of $L_{r}$ in $s$ under $\nu$ is defined as the state that results from the execution of skip in $s$ under $\nu$, that is, $s$ itself as well.

Summary. In all cases, the state $s^{\prime}$ that results from the execution of the rule instance $(r, \nu)$ in $s$ is the same as the state that results from the execution of $L_{r}$ in $s$ under $\nu$. As a consequence, if $s^{\prime}, \nu \models q$, both the correctness formulas for the rule and for the loop-free program derived from it hold; and if $s^{\prime}, \nu \not \neq q$, neither holds.

The fact that a rule and the loop-free program derived from it have the same behavior is consistent with the application of the proof rule (IF) for the conditional statement, to combine under a test consisting of the guard the axioms (ASSIGNMENT) for assignment and (SKIP) for skip.

Another expression of this similarity between a rule and the derived loopfree program is the decomposition of a correctness formula for a rule that can be performed by distinguishing the cases where the rule guard holds or not. In other words, the correctness formula $\{p\} r\{q\}$ for a rule $r=(\vec{o}, \vec{T}, g, a)$ with $a \equiv o_{1} \cdot f_{r}:=e_{r}$, is equivalent to the conjunction of

$$
\{p \wedge g\} a\{q\} \quad \text { and } \quad\{p \wedge \neg g\} \operatorname{skip}\{q\}
$$

that is, by the axioms (ASSIGNMENT) and (SKIP),

$$
p \wedge g \rightarrow q\left[f_{r}\left[o_{1} \mapsto e_{r}\right] / f_{r}\right] \quad \text { and } \quad p \wedge \neg g \rightarrow q .
$$

In the particular cases where the precondition of the correctness formula implies the guard of the rule, or where it is incompatible with the guard of the rule, this gives:

(i) if we have $p \rightarrow g$, then $\{p\} r\{q\}$ is equivalent to $\{p\} a\{q\}$, that is, to $p \rightarrow q\left[f_{r}^{\prime} / f_{r}\right]$ where $f_{r}^{\prime}=f_{r}\left[o_{1} \mapsto e_{r}\right]$;

(ii) if we have $\neg(p \wedge g)$, then $\{p\} r\{q\}$ is equivalent to $\{p\} \operatorname{skip}\{q\}$, that is, to $p \rightarrow q$.

Conversely, in the context of a rule $r=(\vec{o}, \vec{T}, g, a)$, the correctness formula $\{p \wedge g\} a\{q\}$ is equivalent to $\{p\} r\{q \vee p \wedge \neg g\}$.

The equivalences listed above give additional insight into the precise meaning of rule execution. They can also be leveraged to apply the verification method described in Part III in the particular cases to which they relate.

\subsection{Correctness Formula for a Rule Program}

This section defines correctness formulas of the form $\{p\} \mathcal{R}\{q\}$ where $\mathcal{R}$ is a rule program. The pre- and postconditions are closed formulas, expressing assertions on the whole working memory.

The meaning of $\{p\} \mathcal{R}\{q\}$ is standard. For the one-shot execution strategy (where partial and total correctness coincide), it means that any execution of $\mathcal{R}$ started in a state that satisfies $p$ ends in a state that satisfies $q$.

As a first step towards a compositional method for deriving a correctness formula for a rule program from individual correctness formulas for the 
rules in the program, we conclude this section by showing that, in general, a correctness formula $\{p\} r\{q\}$ does not entail the correctness formula $\{\forall \vec{o}: \vec{T} p\} \mathcal{R}\{\forall \vec{o}: \vec{T} q\}$ for the rule program $\mathcal{R}=\{r\}$.

\subsubsection{Syntax and Semantics}

Definition 6.6. A correctness formula for a rule program is a formula of the form $\{p\} \mathcal{R}\{q\}$, where $\mathcal{R}$ is a rule program, and $p$ and $q$ are closed, firstorder, flat, possibly quantified formulas from $\mathcal{F}(\emptyset)$. The formulas $p$ and $q$ are called the pre- and postcondition of the correctness formula, respectively.

As seen in Section 6.2, the pre- and postconditions in a correctness formula for a rule are formulas with free variables. They express assertions on the objects handled by the rule. In contrast, the pre- and postconditions in a correctness formula for a rule program are closed formulas, and they express assertions on the whole working memory.

The semantics of a correctness formula for a rule program is in line with the standard definition [Hoa69, Apt81, AdBO09], including the distinction between partial and total correctness. Indeed, for a general execution strategy, a rule program might not terminate.

Definition 6.7. Let $\{p\} \mathcal{R}\{q\}$ be a correctness formula for a rule program; let $\mathcal{S}$ be a selection strategy and $\mathcal{E}$ be an eligibility strategy. The correctness formula holds in the sense of partial correctness for $\mathcal{S}$ and $\mathcal{E}$ if

for any $\mathcal{T}$-compliant state $s$ such that $s \models p$, any terminating execution of $\mathcal{R}$ from $s$ under the execution strategy $\mathcal{S}, \mathcal{E}$ results in a state $s^{\prime}$ such that $s^{\prime} \models q$.

Definition 6.8. Let $\{p\} \mathcal{R}\{q\}$ be a correctness formula for a rule program; let $\mathcal{S}$ be a selection strategy and $\mathcal{E}$ be an eligibility strategy. The correctness formula holds in the sense of total correctness for $\mathcal{S}$ and $\mathcal{E}$ if

for any $\mathcal{T}$-compliant state $s$ such that $s \models p$, all executions of $\mathcal{R}$ from $s$ under the execution strategy $\mathcal{S}, \mathcal{E}$ terminate, and for each resulting state $s^{\prime}$, one has $s^{\prime} \models q$.

As announced, a correctness formula for a rule program, taken in the sense of partial correctness, means that any execution of the rule program that starts in a state that satisfies the precondition, and that terminates, results in a state that satisfies the postcondition. And when taken in the sense of total correctness, it means that any execution of the rule program that starts in a state that satisfies the precondition terminates, and results in a state that satisfies the postcondition.

Since the one-shot execution strategy (see Section 6.1.1) ensures that all rule program executions terminate, the two notions coincide when under this execution strategy, and we will then omit the distinction.

Example. Consider the rule program $\mathcal{R}=\left\{r_{1}, r_{2}\right\}$ below:

$$
\begin{aligned}
& \mathrm{r}_{1}(\mathrm{x}: \text { Person }): \mathrm{x} \text {.age } \leq 12 \rightarrow \mathrm{x} \text {.status }:=\text { Child } \\
& r_{2}(y: \text { Person }): y \cdot \text { status }=\text { Child } \rightarrow \text { y.gift }:=\text { TeddyBear }
\end{aligned}
$$


Consider the following correctness formula for $\mathcal{R}$ :

$$
\{\forall o: \text { Person (o.age }=10)\} \mathcal{R}\{\forall o: \text { Person } \neg(\text { o.gift }=\text { Champagne })\} .
$$

This correctness formula holds for the one-shot execution strategy. Indeed, consider a $\mathcal{T}$-compliant state $s_{0}$ where the precondition of the correctness formula holds, that is, $s_{0} \models \forall o$ : Person $($ o.age $=10)$. Consider also an execution of $\mathcal{R}$ from $s_{0}$ under the one-shot execution strategy.

The initial configuration for this execution will be $\left\langle E_{0}, s_{0}\right\rangle$, where the set of eligible rule instances will contain all the instances of the rules $r_{1}$ and $r_{2}$ that can be formed with the instances of the type Person, that is, $E_{0}=\{(r, O) \mid$ $r \in \mathcal{R}, O \in \mathcal{M}$ (Person) $\}$. Since $s_{0}$ satisfies the precondition of the correctness formula, the age of all persons is 10, and therefore all instances of $r_{1}$ in $E_{0}$ are applicable. Depending on the status of persons in the state $s_{0}$, which is not constrained by the precondition, the corresponding instances of $r_{2}$ are applicable or not.

An execution of $\mathcal{R}$ from this configuration under the one-shot execution strategy will consist in the application of all the instances of $r_{1}$ and $r_{2}$, since the instances of $r_{2}$ that are initially not applicable will be made applicable by the execution of $r_{1}$ on the same object. The execution of each rule instance makes it ineligible, so each rule instance is executed exactly once. Each application of an instance of $r_{2}$ causes the gift of the corresponding person to be set to TeddyBear, so all executions end in a state where all gifts equal TeddyBear, thus satisfying the postcondition of the correctness formula.

In this example we have chosen the one-shot execution strategy to execute $\mathcal{R}$. The sequential or refraction eligibility strategies would also have made the considered correctness formula valid both in the senses of partial and of total correctness. However, under the identity strategy $\mathcal{E}_{\text {id }}$ (defined in Section 5.6.1), no execution of $\mathcal{R}$ would terminate. The correctness formula would then trivially hold in the sense of partial correctness, and would not hold in the sense of total correctness.

\subsubsection{From Rules to Rule Programs}

Consider a rule $r$, and the rule program $\mathcal{R}=\{r\}$ made of only this rule. Given two assertions $p$ and $q$, we want to compare the correctness formulas $\{p\} r\{q\}$ and $\{p\} \mathcal{R}\{q\}$.

Both when defining the meaning of correctness formulas for rules and of correctness formulas for rule programs, we noted that these meanings were standard. However, these meanings are significantly different from one another. This contrasts with usual programming paradigms, where the meanings of correctness formulas for statements and for programs are close. This peculiarity of rule programs due to the specific way rules are orchestrated in a rule program execution.

We examine these differences below. We also notice that, due to these differences, a correctness formula $\{p\} r\{q\}$ for a rule $r$ does not imply the corresponding correctness formula for a rule program that contains only the rule $r$.

A first, syntactic difference between correctness formulas for rules and for rule programs, is that the pre- and postconditions in a correctness formula for 
a rule are assertions with free variables, whereas those in a correctness formula for a rule program are closed. The former express conditions on the objects handled by the rule, while the latter express conditions on the whole working memory.

The other major difference between correctness formulas for rules and for rule programs, is that for the former only one rule instance execution is considered, whereas for the latter all the rule instance applications that make an execution of the program are considered.

Indeed, on the one hand, the correctness formula $\{p\} r\{q\}$ for the rule expresses that when the rule is executed - that is, when one instance of $r$ is executed - from a state that satisfies $p$, then the resulting state satisfies $q$.

On the other hand, the correctness formula $\{p\} \mathcal{R}\{q\}$ for the rule program (taken in the sense of partial correctness) expresses that when the rule program is executed - that is, since the program contains only one rule, when all the instances of $r$ that are eligible and applicable have been applied - from a state that satisfies $p$, then the states that result from terminating executions satisfy $q$.

This difference in semantics makes it wrong, in the general case, to transpose a correctness formula for a rule into a correctness formula for the program made of only this rule, even once the syntactic concerns are worked out. The proposition below expresses it formally.

Proposition 6.2. Let $\{p\} r\{q\}$ be a correctness formula focused on a rule $r=(\vec{o}, \vec{T}, g, a)$, and let $\mathcal{R}=\{r\}$ be the rule program made of only the rule $r$. Then the correctness formula $\{\forall \vec{o}: \vec{T} p\} \mathcal{R}\{\forall \vec{o}: \vec{T} q\}$ does not hold for the one-shot execution strategy. In other words, the proof rule below is not sound

$$
\frac{\{p\} r\{q\}}{\{\forall \vec{o}: \vec{T} p\} \mathcal{R}\{\forall \vec{o}: \vec{T} q\}} \quad \text { where } \mathcal{R}=\{r\}
$$

The notation $\forall \vec{o}: \vec{T}$ used above is an abbreviation for $\forall o_{1}: T_{1} \ldots \forall o_{n}: T_{n}$.

Proof. To prove the proposition, we exhibit a counterexample. Consider the rule program $\mathcal{R}=\{r\}$ made of the only rule below:

$$
r(\mathrm{c}: \text { Cart, } \mathrm{p}: \text { Person }): \mathrm{p} . \text { age }=21 \rightarrow \mathrm{c} . \text { value }:=\mathrm{c} . \text { value }+10
$$

The correctness formula $\{$ p.age $=21 \wedge$ c.value $=0\} r\{$ c.value $=10\}$ holds for this rule. We are going to show that the correctness formula

$\{\forall c:$ Cart $\forall p:$ Person $(p . a g e=21 \wedge$ c.value $=0)\} \mathcal{R}$

$$
\{\forall c \text { : Cart } \forall p: \text { Person }(\text { c.value }=10)\}
$$

does not hold for the rule program $\mathcal{R}$.

Consider the following setting:

$$
\begin{aligned}
& \mathcal{M}(\text { Person })=\{\text { Alice }, \text { Bob }\} \\
& \mathcal{M}(\text { Cart })=\{\text { Caddie }\} \\
& s_{0}=\quad \text { age }^{s_{0}}: \text { Alice } \mapsto 21 \quad \text { value }^{s_{0}}: \text { Caddie } \mapsto 0 . \\
& \text { Bob } \mapsto 21
\end{aligned}
$$


With this setting one has $s_{0} \models \forall c$ : Cart $\forall p$ :Person $(p$.age $=21 \wedge c$.value $=0)$.

An execution of $\mathcal{R}$ from $s_{0}$ is

$$
s_{0} \stackrel{(r, \text { Caddie }, \text { Alice })}{\longrightarrow} s_{1} \stackrel{(r, \text { Caddie }, \text { Bob })}{\longrightarrow} s_{2} .
$$

In this execution, we have value $e^{s_{1}}$ (Caddie $)=10$ and value $e^{s_{2}}$ (Caddie $)=20$. As a consequence, the final state $s_{2}$ of this execution does not satisfy

$$
\forall c: \text { Cart } \forall p: \text { Person (c.value }=10) \text {. }
$$

The reason behind this negative result is that among the multiple rule instance applications in a rule program execution, some might interfere with the pre- and postconditions of the correctness formula, hence breaking its validity.

It is the subject of Part III to study under which conditions individual correctness formulas focused on the rules of a program can be leveraged to prove a correctness formula for the rule program itself. 


\section{Chapter 7}

\section{Correctness of Programs: A Comparison}

In the previous chapter, we studied the meaning of a rule program from the viewpoint of the relation between the initial and final states of its possible executions. We used correctness formulas of the form $\{p\} \mathcal{R}\{q\}$ to embody this meaning.

In the present chapter, we compare rule programs with parallel programs and with nondeterministic programs. In these comparisons, we study whether a given rule program can, in general, be simulated by a parallel or a nondeterministic program, in the sense that both would satisfy the same correctness formulas.

The outcome is that, in general, a rule program cannot be simulated by a parallel program. This implies that a rule program cannot be verified by a mere translation into a parallel one, on which the existing verification methods for parallel programs [OG76, Hoa76] would then be applied.

On the other hand, a rule program can always be simulated by a nondeterministic program. However, the verification method for nondeterministic programs based on a dedicated Hoare logic [Apt84] is not compositional, as it relies on a global invariant.

These results motivate the search for a compositional method to derive a correctness formula for a rule program out of individual correctness formulas for its rules. This search is the subject of the next chapters.

\subsection{Correctness of Loop-Free Parallel Programs}

Section 6.3.2 showed that a rule satisfies the same correctness formulas as its derived loop-free program (if $g$ then $a$ else skip fi). This section determines that a rule program and the parallel program composed of the loop-free programs derived from its rules might have different behaviors.

Furthermore, it shows that, in general, there does not exist a parallel program with loop-free processes that has the same behavior as a given rule program. This core reason is that parallel programs with loop-free processes might fail to take into account the activation of a rule by another. 
To compare rule programs with parallel programs, the standard interleaving semantics of parallel programs is extended into a saturated interleaving semantics. Under this saturated interleaving semantics, parallel programs can be executed on a set of objects such as the working memory.

As mentioned in Chapter 5, rule programs are declarative programs composed of rules that are combined by a rule engine. It is thus natural to draw an analogy with parallel programs, which are composed of processes combined by a scheduler.

In this section, we compare rule programs with parallel ones composed of loop-free processes. In the next section, we compare them with parallel programs that admit while programs as their processes. We show in particular that in general there is no parallel program (of a precise form) with the same behavior as a rule program.

\subsubsection{Loop-Free Parallel Programs}

\section{Syntax}

We consider parallel programs built the classical way [Hoa76, AdBO09], that is, by assembling processes with a parallel composition control structure. Each process is a loop-free program as defined by the grammar of the language $\mathcal{L}(V)$ given in Fig. 6.1, augmented with a new derivation for statements that introduces the atomic region. In Fig. 7.1, we provide this rule and the grammar for the $\mathcal{P}_{\mathcal{L}}(V)$ language of parallel programs with loop-free processes.

$$
\begin{aligned}
& \langle\text { loop-free stat. }\rangle::=\text { '〈' }\langle\text { loop-free pgm. }\rangle \text { ' }\rangle \text { ' } \\
& \left\langle\text { lf-par. pgm. : } \mathcal{P}_{\mathcal{L}}(V)\right\rangle::=\text { '[' }\langle\text { lf-processes }\rangle \text { ']' } \\
& \langle\text { lf-processes }\rangle::=\langle\text { lf-process : } \mathcal{L}(V)\rangle \\
& \text { | }\langle\text { lf-process : } \mathcal{L}(V)\rangle^{\prime} \| \text { ' }\langle\text { lf-processes }\rangle
\end{aligned}
$$

Figure 7.1: Parallel programs with loop-free processes.

A parallel program inherits the type constraints on the variables of its component processes.

Example. The program $L=\left[L_{1} \| L_{2}\right]$ is a parallel program with two loop-free processes:

$$
\begin{gathered}
\mathrm{L}_{1}(\mathrm{x}: \text { Person }): \mathrm{x} \cdot \text { bonus }:=0 \\
\mathrm{~L}_{2}(\mathrm{x}: \text { Person, } \mathrm{y}: \text { Person }): \mathrm{y} \cdot \text { bonus }:=\mathrm{x} \cdot \text { bonus }+10
\end{gathered}
$$

\section{Classical interleaving semantics}

In the widely used interleaving semantics for parallel programs, the executions of a parallel program are obtained by combining the execution of the statements in the processes in all the possible manners that respect the control flow of each process.

In this semantics, when a parallel program is executed on some input data, the processes are first instantiated on the data, that is, variables are given 
the value provided as input to the program, and then all combinations of the statements of the process instances are formed.

For example, consider the parallel program $L=\left[L_{1} \| L_{2}\right]$ defined in the example above. Consider also a valuation that maps the variable $x$ onto the value Alice, and the variable $y$ onto Bob. Finally, consider an execution of $L$ on the input data modeled by this valuation and from the state $s_{0}$ defined below:

$$
\text { bonus }^{s_{0}}(\text { Alice })=30 \quad \text { bonus }^{s_{0}}(\mathrm{Bob})=20
$$

The program $L$ then admits the following two executions:

$$
\begin{aligned}
& s_{0} \underset{1^{\text {st }} \text { statement }}{\stackrel{\left(L_{1}, \text { Alice }\right)}{\longrightarrow}} \begin{array}{c}
\text { bonus }(\text { Alice })=0 \\
\text { bonus }(\text { Bob })=20
\end{array} \stackrel{\left(L_{2}, \text { Alice, Bob }\right)}{\stackrel{1^{\text {st }} \text { statement }}{\longrightarrow}} \begin{array}{l}
\text { bonus }(\text { Alice })=0 \\
\text { bonus }(\text { Bob })=10
\end{array} \\
& s_{0} \underset{1^{\text {st }}}{\stackrel{\left(L_{2}, \text { Alice }, \text { Bob }\right)}{\longrightarrow}} \quad \begin{array}{lll}
\text { bonus }(\text { Alice })=30 & \stackrel{\left(L_{1}, \text { Alice }\right)}{\longrightarrow} & \text { bonus }(\text { Alice })=0 \\
1^{\text {st }} \text { statement } & \text { bonus }(\text { Bob })=40
\end{array}
\end{aligned}
$$

\section{Saturated interleaving semantics}

The semantics for parallel processes that we are introducing here does not differ with the classic interleaving semantics in the way the executions of the rule program are obtained, as far as the combination of process statements is concerned. Its difference lies in the way the processes are instantiated on input data before the combination occurs. On this point, it reproduces the instantiation of rules into rule instances by the rule engine.

In the saturated interleaving semantics, a parallel program $L=\left[L_{1}\|\ldots\| L_{n}\right]$ is considered for execution on a working memory $\mathcal{M}$. For each $i=1, \ldots, n$, the process $L_{i}$ involves variables in its text and comes with a type constraint $\left(\vec{o}_{i}: \vec{T}_{i}\right)$ for them.

To instantiate the processes, the classic interleaving semantics finds in the input data the (single) value to give to each variable of the program. Instead, the saturated interleaving semantics considers, for a process $L_{i}$, all the instances of the type $T_{j}^{i}$ as possible values of the variable $o_{j}^{i}$, and creates as many instances of the process $L_{i}$. That is, it creates one instance of $L_{i}$ for each tuple of objects in the working memory that is well-typed for $\left(\vec{o}_{i}: \vec{T}_{i}\right)$.

For example, consider a parallel program $L=\left[L_{1} \| L_{2}\right]$ composed of two processes, where $L_{1}$ involves the variables $p$ : Person and $c$ : Cart, and $L_{2}$ involves the variable $q$ :Person. In an execution of $L$ on a working memory with the instances $\mathcal{M}($ Person $)=\{$ Alice, Bob $\}$ and $\mathcal{M}($ Cart $)=\{$ Caddie $\}$, the following process instances are created:

$$
\begin{array}{rr}
\left(L_{1}, \text { Alice }, \text { Caddie }\right) & \left(L_{2}, \text { Alice }\right) \\
\left(L_{1}, \text { Bob }, \text { Caddie }\right) & \left(L_{2}, \text { Bob }\right) .
\end{array}
$$

The statements of all the process instances are then combined to form the executions of the parallel program. Assuming that each process of the example just above contains only one statement, the combination of the four process instances gives $4 !=24$ possible executions of $L$, although these executions might represent fewer pairs of initial and final states.

The saturated interleaving semantics for parallel programs is well-suited to compare a rule program with a parallel program, because it provides a meaning for the execution of a parallel program on a working memory. It does so 
by transposing to parallel programs the way in which a rule engine instantiates rules. The specificity of the parallel programming paradigm, namely the interleaving semantics, is preserved.

The classic and saturated interleaving semantics coincide when the working memory contains exactly one instance of each type involved in the program. Each process then yields only one instance.

\subsubsection{Loop-Free Parallel Program Derived from a Rule Program}

We have defined in Section 6.3.2 that the loop-free program derived from a rule $r=(\vec{o}, \vec{T}, g, a)$ is the program $L_{r}(\vec{o}: \vec{T})$ : if $g$ then $a$ else skip fi.

Definition 7.1. Given a rule program $\mathcal{R}=\left\{r_{1}, \ldots, r_{n}\right\}$, the parallel program with loop-free processes derived from $\mathcal{R}$, is the program $L_{\mathcal{R}} \equiv$ $\left[\left\langle L_{r_{1}}\right\rangle\|\ldots\|\left\langle L_{r_{n}}\right\rangle\right]$ composed of the loop-free programs derived from the rules of $\mathcal{R}$, each treated as an atomic region.

Example. Consider the rule program $\mathcal{R}=\left\{r_{1}, r_{2}\right\}$ composed of the rules below:

$$
\begin{aligned}
& \mathrm{r}_{1}(\mathrm{p}: \text { Person }): \mathrm{p} . \text { age }>21 \rightarrow \text { p.bonus }:=10 \\
& \quad \mathrm{r}_{2}(\mathrm{c}: \text { Car }): \text { c.mileage }<100000 \rightarrow \text { c.score }:=15
\end{aligned}
$$

The parallel program with loop-free processes derived from $\mathcal{R}$ is the program $L_{\mathcal{R}} \equiv\left[\left\langle L_{r_{1}}\right\rangle \|\left\langle L_{r_{2}}\right\rangle\right]$, with

$$
\begin{aligned}
& \mathrm{L}_{\mathrm{r}_{1}}(\mathrm{p}: \text { Person }): \text { if } \mathrm{p} . \text { age }>21 \text { then p.bonus }:=10 \text { else skip fi } \\
& \quad \mathrm{L}_{\mathrm{r}_{2}}(\mathrm{c}: \text { Car }): \text { if c.mileage }<100000 \text { then c.score }:=15 \text { else skip fi }
\end{aligned}
$$

The natural question that arises is whether, given a rule program $\mathcal{R}$, the derived program $L_{\mathcal{R}}$ has the same behavior, in the sense that its executions under the saturated interleaving semantics make the same correctness formulas valid. The answer is: in general, no.

In the example just above, $\mathcal{R}$ and $L_{\mathcal{R}}$ have the same behavior, but this is a consequence of the fact that the rules in $\mathcal{R}$ (and hence the processes in $L_{\mathcal{R}}$ ) are disjoint, in the sense that no attribute updated by one of the rules occurs in the other rule.

Proposition 7.1. There exist rule programs $\mathcal{R}$ such that the parallel program with loop-free processes $L_{\mathcal{R}}$ derived from $\mathcal{R}$ does not satisfy the same correctness formulas as $\mathcal{R}$.

Proof. Consider the rule program $\mathcal{R}=\left\{r_{1}, r_{2}\right\}$ composed of the rules below:

$$
\begin{aligned}
& r_{1}(x: T): x \cdot a=1 \rightarrow x \cdot b:=1 \\
& r_{2}(x: T): x \cdot b=1-x \cdot c:=1
\end{aligned}
$$

The parallel program with loop-free processes derived from $\mathcal{R}$ is the program $L_{\mathcal{R}} \equiv\left[\left\langle L_{r_{1}}\right\rangle \|\left\langle L_{r_{2}}\right\rangle\right]$, with

$$
\begin{aligned}
& \mathrm{L}_{\mathrm{r}_{1}}(\mathrm{x}: \mathrm{T}): \text { if } \mathrm{x} \cdot \mathrm{a}=1 \text { then } \mathrm{x} \cdot \mathrm{b}:=1 \text { else skip } \mathrm{fi} \\
& \mathrm{L}_{\mathrm{r}_{2}}(\mathrm{x}: \mathrm{T}): \text { if } \mathrm{x} \cdot \mathrm{b}=1 \text { then } \mathrm{x} \cdot \mathrm{c}:=1 \text { else skip } \mathrm{fi}
\end{aligned}
$$


The proof is based on the fact that the rule program satisfies the following correctness formula, whereas the derived parallel program would not:

$$
\{\forall x: T(x . a=1 \wedge x . b=0 \wedge x . c=0)\} \mathcal{R}\{\forall x: T(x . a=1 \wedge x . b=1 \wedge x . c=1)\} .
$$

During the whole proof, we limit ourselves to executions of $\mathcal{R}$ and $L_{\mathcal{R}}$ on a working memory reduced to one object; since the difference of behavior is visible under this limitation, it suffices to prove the proposition.

Executions of $\mathcal{R}$. The initial configuration of the rule engine is $\left\langle E_{0}, s_{0}\right\rangle$, with

$$
\begin{aligned}
\mathcal{M}(\mathrm{T}) & =\{\mathrm{X}\} \\
E_{0} & =\left\{\left(r_{1}, \mathrm{X}\right),\left(r_{2}, \mathrm{X}\right)\right\} \\
s_{0} & =a^{s_{0}}: \mathrm{X} \mapsto 1 \quad b^{s_{0}}: \mathrm{X} \mapsto 0 \quad c^{s_{0}}: \mathrm{X} \mapsto 0 .
\end{aligned}
$$

In this initial configuration, the instances of $r_{1}$ and $r_{2}$ on $\mathrm{X}$ are eligible, but only $\left(r_{1}, \mathrm{X}\right)$ is applicable.

The only possible transition is by $\left(r_{1}, \mathrm{X}\right)$ to a configuration $\left\langle E_{1}, s_{1}\right\rangle$, where $E_{1}=\left\{\left(r_{2}, \mathrm{X}\right)\right\}$ and $a^{s_{1}}(\mathrm{X})=b^{s_{1}}(\mathrm{X})=1$ while $c^{s_{1}}(\mathrm{X})=0$. In this configuration, both $\left(r_{1}, \mathrm{X}\right)$ and $\left(r_{2}, \mathrm{X}\right)$ are applicable, but only the latter is also eligible.

The only possible transition is by $\left(r_{2}, \mathrm{X}\right)$ to a configuration $\left\langle E_{2}, s_{2}\right\rangle$, where $E_{2}=\emptyset$ and $a^{s_{2}}(\mathrm{X})=b^{s_{2}}(\mathrm{X})=c^{s_{2}}(\mathrm{X})=1$. The execution of $\mathcal{R}$ ends in this configuration, in which the postcondition of the correctness formula holds.

Executions of $L_{\mathcal{R}}$. Since the working memory is reduced to $\mathcal{M}(\mathrm{T})=\{\mathrm{X}\}$, the process instances are simply $\left(\left\langle L_{r_{1}}\right\rangle, \mathrm{X}\right)$ and $\left(\left\langle L_{r_{2}}\right\rangle, \mathrm{X}\right)$. Since each process is composed of an atomic region, there are two executions of $L_{\mathcal{R}}$, corresponding to the execution of the two process instances in one order or the other.

Let us consider the execution in which $\left(\left\langle L_{r_{2}}\right\rangle, \mathrm{X}\right)$ is executed first, from the state $s_{0}$ described above. In this execution, the loop-free program $L_{2}$ takes the else branch and executes skip. The resulting state is therefore $s_{0}$ itself. The process instance $\left(\left\langle L_{r_{1}}\right\rangle, \mathrm{X}\right)$ is then executed, with the else branch of the conditional, resulting in the final state $s_{1}$ where $a^{s_{1}}(\mathrm{X})=b^{s_{1}}(\mathrm{X})=1$, but $c^{s_{1}}(\mathrm{X})=0$. The correctness formula is thus not satisfied.

\subsubsection{Rule Programs vs. Loop-Free Parallel Programs}

We have just seen that the parallel program with loop-free processes $L_{\mathcal{R}}$ derived from a rule program $\mathcal{R}$ is not a good candidate to reproduce the behavior of $\mathcal{R}$. The next proposition expresses that, in general, there does not exist a parallel program with loop-free processes (obeying a few reasonable syntactic constraints) that would reproduce the behavior of a given rule program.

Proposition 7.2. There are rule programs of the form $\mathcal{R}=\left\{r_{1}, \ldots, r_{n}\right\}$, for which no parallel program $L \equiv\left[L_{1}\|\ldots\| L_{n}\right]$ satisfies the same correctness formulas as the rule program, and where $L_{i} \in \mathcal{L}\left(\operatorname{var}\left(r_{i}\right)\right)$ with $\operatorname{Attr}\left(L_{i}\right) \subseteq \operatorname{Attr}\left(r_{i}\right)$ for $i=1, \ldots, n$.

The requirement $L_{i} \in \mathcal{L}\left(\operatorname{var}\left(r_{i}\right)\right)$ means that $L_{i}$ is a loop-free program whose variables are taken among those of the rule $r_{i}$. In addition, $\operatorname{Attr}\left(L_{i}\right) \subseteq \operatorname{Attr}\left(r_{i}\right)$ means that the attribute symbols used in $L_{i}$ are also taken among those of $r_{i}$. 
Proof. To prove the proposition, we consider a specific rule program and we prove by contradiction that there exists no parallel program with the same behavior.

Consider again the rule program $\mathcal{R}=\left\{r_{1}, r_{2}\right\}$ composed of the rules below:

$$
\begin{aligned}
& r_{1}(x: T): x \cdot a=1 \rightarrow x \cdot b:=1 \\
& r_{2}(x: T): x \cdot b=1 \rightarrow x \cdot c:=1
\end{aligned}
$$

Assume that a program $L=\left[L_{1} \| L_{2}\right]$ exists, where $L_{1} \in \mathcal{L}(\{x\})$ and $L_{2} \in$ $\mathcal{L}(\{x\})$, with $\operatorname{Attr}\left(L_{1}\right) \subseteq\{a, b\}$ and $\operatorname{Attr}\left(L_{2}\right) \subseteq\{b, c\}$, and satisfying the same correctness formulas as $\mathcal{R}$.

Consider the executions of $L$ on a working memory $\mathcal{M}(\mathrm{T})=\{\mathrm{X}\}$ reduced to one object. Since $L_{1}$ and $L_{2}$ are loop-free programs, there exists an execution of $L$ in which $\left(L_{2}, \mathrm{X}\right)$ terminates before $\left(L_{1}, \mathrm{X}\right)$ starts. Let us call $s_{1}$ the state at this point, and $s$ and $s^{\prime}$ the initial and final states of this execution. Therefore, we have

$$
s \stackrel{\left(L_{2}, \mathrm{X}\right)}{\longrightarrow} s_{1} \stackrel{\left(L_{1}, \mathrm{X}\right)}{\longrightarrow} s^{\prime} .
$$

Since $L_{1}$ cannot modify the values of the $c$ attribute, the value of this attribute on $\mathrm{X}$ is the same in $s_{1}$ and at the end of the execution. That is, $c^{s^{\prime}}(\mathrm{X})=c^{s_{1}}(\mathrm{X})$.

On the other hand, since $L_{2}$ is deterministic and does not involve the $a$ attribute, the value $c^{s_{1}}(\mathrm{X})$ is the same if

and if

$$
a^{s}(\mathrm{X})=0 \quad b^{s}(\mathrm{X})=1 \quad c^{s}(\mathrm{X})=0
$$

$$
a^{s}(\mathrm{X})=1 \quad b^{s}(\mathrm{X})=1 \quad c^{s}(\mathrm{X})=0 .
$$

Yet the final value of $c$ on $\mathrm{X}$ is not the same when $\mathcal{R}$ is executed from these two initial states. This contradicts the assumption that $L$ and $\mathcal{R}$ satisfy the same correctness formulas.

\subsection{Correctness of Parallel Programs}

Since parallel programs with loop-free processes suffer from terminating too soon, this section considers while programs instead of loop-free programs as processes of parallel programs. To help modeling the execution of rule programs as performed by the abstract machine introduced in Chapter 5, we allow the parallel programs to use ghost variables (Section 7.2.1).

Again, we conclude that, in general, there does not exist a parallel program (even with while programs as processes and including ghost variables) that has the same behavior as a given rule program. This motivates the development of a specific verification method for rule programs, as opposed, for example, to the reuse of an existing verification method for parallel programs such as the one proposed by Owicki and Gries [OG76].

In this section, we compare rule programs with parallel programs with while programs as their processes. In addition to not being loop-free, parallel programs with while programs as their processes also contain additional variables, called ghost variables. 
We start the section with the definition of ghost variables, which can be used to encode information that is otherwise kept implicit in the rule engine. Based on this definition, we introduce while programs and parallel programs using while programs as their processes. Then, we compare rule programs with the parallel programs that can be derived from them in a similar way as in Section 7.1.2. Finally, we compare rule programs with general parallel programs with while programs as their processes.

\subsubsection{Ghost Variables}

The purpose of ghost variables is to allow imperative programs, such as while programs, to encode information that is implicit in rule programs because it is maintained internally by the rule engine. This information is analogous to the program counter maintained by the processor that executes a sequential program.

More generally, a ghost variable is used to store information in addition to the information encoded by attributes of objects. Contrary to attributes, ghost variables do not take object values but only Boolean or theory-specific values, such as numbers.

In the context of parallel programs, each ghost variable is local to a process. There are no shared ghost variables. (An extension with shared additional variables is discussed at the end of Section 7.2.5, but we do not include these in the definition of ghost variables.) One consequence is that during the execution of a parallel program under the saturated interleaving semantics, ghost variables undergo the same instantiation scheme as the processes in which they occur, leading to as many replicates of the ghost variable as there are instances of the process. From this perspective, ghost variables are in fact functions, and each process instance handles the image by this function of the objects on which the process is instantiated.

\section{Syntax}

We augment the signature $\Sigma$ introduced in Section 3.1 with an infinite set of function symbols, noted Ghost. We will use lowercase Greek letters for ghost variables, such as $\gamma, \delta, \ldots$

We add a second parameter to all the languages defined up to now with one parameter. These languages are: $\mathcal{E}(V), \mathcal{B}(V), \mathcal{F}(V)$ introducing respectively expressions, Boolean expressions or non-quantified formulas, and possibly quantified formulas in Fig. 3.1; $\mathcal{A}(V)$ introducing assignment in Fig. 3.3; $\mathcal{L}(V)$ for loop-free programs in Fig. 6.1; and $\mathcal{P}_{\mathcal{L}}(V)$ for parallel programs with loop-free processes in Fig. 7.1.

The second parameter comes in addition to the set $V \subseteq$ Var of variables, and is a set of ghost variables $G \subseteq$ Ghost. We define the languages $\mathcal{E}(V, G)$ and $\mathcal{A}(V, G)$ by the grammar in Fig. 7.2. This grammar simply adds derivations to incorporate ghost variables into expressions and assignment to the grammars of $\mathcal{E}(V)$ and $\mathcal{A}(V)$. (Similar extensions have to be made for each theory-specific kind of expression.)

The $\mathcal{B}(V, G), \mathcal{F}(V, G), \mathcal{L}(V, G)$ and $\mathcal{P}_{\mathcal{L}}(V, G)$ languages are obtained by replacing expressions and assignments in their grammars with those from $\mathcal{E}(V, G)$ 


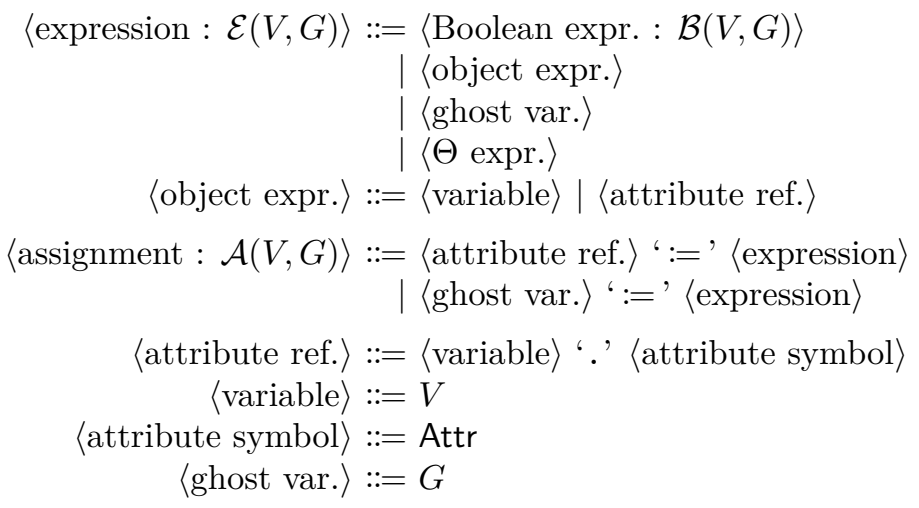

Figure 7.2: Expressions and assignment with ghost variables.

and $\mathcal{A}(V, G)$. Without surprise, the one-parameter version of each of these languages is equivalent to its two-parameter version where the set of ghost variables is empty: for example, the languages $\mathcal{L}(V)$ and $\mathcal{L}(V, \emptyset)$ are the same.

Example. The following program contains the ghost variable $\gamma$ :

$\mathrm{L}(\mathrm{p}:$ Person $): \gamma:=$ false if $\gamma$ then p.bonus $:=0$ else $\mathrm{p}$. bonus $:=1 \mathrm{fi}$

Although ghost variables are included in the signature as function symbols, a ghost variable appears in a program only with its symbol. Its implicit argument list is made of the variables of the program in which it appears.

\section{Semantics}

A state $s$ interprets a ghost variable $\gamma$ occurring in a program that involves the variables $o_{1}, \ldots, o_{m}$, by a function $\gamma^{s}$ defined on $\mathbb{D}^{m}$ with values in $\mathbb{V} \cup\{\perp\}$. A ghost variable is eventually given a value by an additional variable valuation. The value of $\gamma$ in the state $s$ under the valuation $\nu$ is $\gamma^{s}\left(\nu\left(o_{1}\right), \ldots, \nu\left(o_{m}\right)\right)$.

Assigning an expression $e$ to a ghost variable $\gamma$ in a state $s$ and under a valuation $\nu$ results in a state $s^{\prime}$, where the ghost variable is interpreted by a function $\gamma^{s^{\prime}}$ that has the same values as $\gamma^{s}$, except on $\left(\nu\left(o_{1}\right), \ldots, \nu\left(o_{m}\right)\right)$ where it takes the value resulting from the interpretation of $e$ in $s$ under $\nu$.

\subsection{2 while Programs}

We now introduce the languages $\mathcal{W}(V, G)$ of while programs, parameterized by a set $V \subseteq$ Var of variables and a set $G \subseteq$ Ghost of ghost variables.

The grammar of $\mathcal{W}(V, G)$ in Fig. 7.3 relies on the languages of expressions, formulas and assignment extended with ghost variables in Fig. 7.2. It builds on the grammar of loop-free programs introduced in Fig. 6.1, adding the while statement. Note that although while loops may occur within other loops or within conditionals, they may not occur within atomic regions.

Also, if $b \in \mathcal{B}(V, G)$ is a Boolean expression, and $L \in \mathcal{L}(V, G)$ is a loopfree program, we will use while $b\langle L\rangle$ as an abbreviated notation for the loop while $b$ begin $\langle L\rangle$ end. 


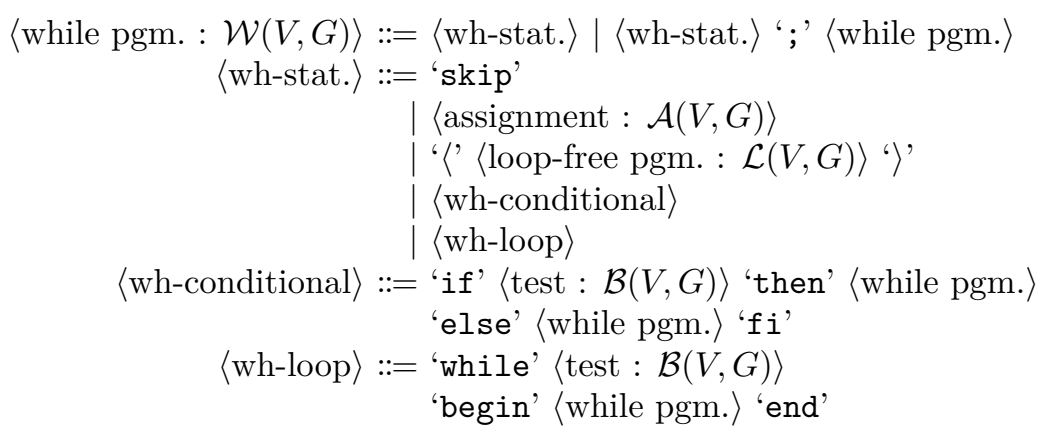

Figure 7.3: while Programs.

We define that a while program is well-typed for a type constraint by induction on its structure.

The execution of a loop while $b$ begin $W$ end in a state $s$ under a valuation $\nu$, where $b$ is a Boolean expression and $W$ is a while program, is composed of a possibly infinite sequence of transitions between states. This sequence starts in $s$; if $b$ does not hold in $s$ under $\nu$ it ends, and the state resulting from the execution of the loop is $s$. Otherwise, the transition sequence extends from $s$ by executing the program $W$, resulting in a state $s^{\prime}$. If $b$ does not hold in $s^{\prime}$ under $\nu$ the transition sequence ends, and the state resulting from the execution of the loop is $s^{\prime}$, etc.

Given a while program $W \in \mathcal{W}$ (Var, Ghost) well-typed for some type constraint $\tau$, and two $\mathcal{T}$-compliant states $s$ and $s^{\prime}$, we note $s \stackrel{W}{\longrightarrow} s^{\prime}$ if there exists a valuation $\nu$ well-typed for $\tau$, such that the execution of $W$ in $s$ under $\nu$ terminates and results in $s^{\prime}$.

\subsubsection{Parallel Programs}

We can now introduce the language $\mathcal{P}_{\mathcal{W}}(V, G)$ of parallel programs composed of while programs with ghost variables as their processes. Its grammar is provided in Fig. 7.4.

$$
\begin{aligned}
\left\langle\text { wh-par. pgm. : } \mathcal{P}_{\mathcal{W}}(V, G)\right\rangle::= & \text { '['〈wh-processes }\rangle \text { ' }] \\
\langle\text { wh-processes }\rangle: & \langle\langle\text { wh-process }\rangle \\
& \mid\langle\text { wh-process }\rangle \text { ' } \| \text { ' }\langle\text { wh-processes }\rangle \\
\langle\text { wh-process }\rangle::=\mathcal{W}(V, G) &
\end{aligned}
$$

Figure 7.4: Parallel programs composed of while programs.

We will use the saturated interleaving semantics for parallel programs. We defined this semantics in Section 7.1.1 on parallel programs with loop-free processes; it transposes directly onto parallel programs composed of while programs with ghost variables.

Example. Consider the parallel program $P=\left[P_{1}\right]$ with only one process, as defined below.

$$
\begin{aligned}
\mathrm{P}_{1}(\mathrm{p}: \text { Person }): & \gamma:=\text { true } \\
& \text { if } \gamma \text { then } \text { p.bonus }:=0 ; \gamma:=\text { false else } \mathrm{p} . \text { bonus }:=1 \mathrm{fi}
\end{aligned}
$$


An execution of $P$ on a working memory with two persons $\mathcal{M}$ (Person) $=$ $\{$ Alice, Bob)\} will interleave the statements of two process instances, namely $\left(P_{1}\right.$, Alice $)$ and $\left(P_{1}, \mathrm{Bob}\right)$. In each of these process instances, the ghost variable $\gamma$ will be interpreted on the person specific to the instance. As a consequence, the statement $\gamma:=$ false in one process instance, say $\left(P_{1}\right.$, Alice), will update only the value of $\gamma$ on Alice.

In other words, and as mentioned earlier, ghost variables are local to each process instance; there are no shared ghost variables. Process instances may still share data through attributes of objects, although in this example this cannot happen because the process has only one variable. As a consequence, the two process instances are disjoint, and the program $P$ has only one execution, which sets the bonuses of Alice and Bob to zero.

\subsubsection{Parallel Program Derived from a Rule Program}

We have seen in Proposition 7.1 that, in general, the parallel program with loopfree processes $L_{\mathcal{R}}$ derived from a rule program $\mathcal{R}$ has not the same behavior as $\mathcal{R}$, basically because the loop-free process derived from one rule might terminate before the rule guard has the chance of being made valid by the execution of another rule/process.

With the introduction of the while statement, we are now able to propose an alternate parallel program derived from a rule program that will not terminate prematurely. We must however prevent this program from entering trivial loops when the application of a rule does not invalidate its guard. This task is the role of the eligibility strategy in a rule engine.

The one-shot eligibility strategy makes a rule instance ineligible once it has been applied (see Section 5.6.4). To encode it, we use for each rule a ghost variable $\delta$ (for "done") with Boolean values.

Definition 7.2. Given a rule program $\mathcal{R}=\left\{r_{1}, \ldots, r_{n}\right\}$, the parallel program with while programs as processes derived from $\mathcal{R}$, is the program $W_{\mathcal{R}} \equiv$ $\left[W_{r_{1}}\|\ldots\| W_{r_{n}}\right]$ defined as follows, with $r_{i}=\left(\vec{o}_{i}, \vec{T}_{i}, g_{i}, a_{i}\right)$ and $\delta_{i} \in$ Ghost for $i=1, \ldots, n$ :

$$
W_{r_{i}}\left(\vec{o}_{i}: \vec{T}_{i}\right) \stackrel{\text { def }}{\equiv} \delta_{i}:=\text { false; while } \neg \delta_{i}\left\langle\text { if } g_{i} \text { then } a_{i} ; \delta_{i}:=\text { true else skip } \mathrm{fi}\right\rangle
$$

Example. Let us consider the program taken as counter-example in Proposition 7.1:

$$
\begin{aligned}
& r_{1}(x: T): x \cdot a=1 \rightarrow x \cdot b:=1 \\
& r_{2}(x: T): x \cdot b=1 \rightarrow x \cdot c:=1
\end{aligned}
$$

The parallel program with while programs as processes derived from $\mathcal{R}$ is the program $W_{\mathcal{R}} \equiv\left[W_{r_{1}} \| W_{r_{2}}\right]$, with

$$
\begin{aligned}
W_{r_{1}}(x: T) \equiv & \delta_{1}:=\text { false; } \\
& \text { while } \neg \delta_{1}\left\langle\text { if } x . a=1 \text { then } x . b:=1 ; \delta_{1}:=\text { true else skip } \mathrm{fi}\right\rangle \\
W_{r_{2}}(x: T) \equiv & \delta_{2}:=\text { false; } \\
& \text { while } \neg \delta_{2}\left\langle\text { if } x . b=1 \text { then } x . c:=1 ; \delta_{2}:=\text { true else skip } \mathrm{fi}\right\rangle
\end{aligned}
$$


As in the proof of Proposition 7.1, let us consider the execution of $W_{\mathcal{R}}$ from the state $s_{0}$ below:

$$
\begin{aligned}
\mathcal{M}(\mathrm{T}) & =\{\mathrm{X}\} \\
s_{0} & =a^{s_{0}}: \mathrm{X} \mapsto 1 \quad b^{s_{0}}: \mathrm{X} \mapsto 0 \quad c^{s_{0}}: \mathrm{X} \mapsto 0 .
\end{aligned}
$$

The execution of the parallel program that caused the mismatch in behavior was the one in which the process derived from $r_{2}$ was executed first. In such an execution, with $\left(W_{r_{2}}, \mathrm{X}\right)$ starting while we still have $b^{s_{0}}(\mathrm{X})=0$, the process instance $\left(W_{r_{2}}, \mathrm{X}\right)$ would loop on the skip statement.

Assuming a fair scheduler, $\left(W_{r_{1}}, \mathrm{X}\right)$ would eventually be started; this would cause a transition to a state $s_{1}$ where $b^{s_{1}}(\mathrm{X})=1$ and $\delta_{1}^{s_{1}}(\mathrm{X})=$ true.

The process instance $\left(W_{r_{1}}, \mathrm{X}\right)$ would then terminate, whereas $\left(W_{r_{2}}, \mathrm{X}\right)$ would cause a transition to a state $s_{2}$ where $c^{s_{2}}(\mathrm{X})=1$ and $\delta_{2}^{s_{2}}(\mathrm{X})=$ true.

With the process instance $\left(W_{r_{2}}, \mathrm{X}\right)$ terminating in turn, the program $W_{\mathcal{R}}$ would end in a state that satisfies the expected postcondition.

The program $W_{\mathcal{R}}$ is thus a better candidate than $L_{\mathcal{R}}$ to reproduce the behavior of $\mathcal{R}$. However, it fails to do so from the standpoint of termination.

Proposition 7.3. Let $W_{\mathcal{R}}$ be the parallel program with while programs as processes derived from a rule program $\mathcal{R}$ :

(i) Any terminating execution of $W_{\mathcal{R}}$ is also an execution of $\mathcal{R}$.

(ii) $W_{\mathcal{R}}$ might not terminate (whereas $\mathcal{R}$ always does).

This proposition can informally be worded as " $W_{\mathcal{R}}$ has the same behavior as $\mathcal{R}$ when it terminates". To prove it, we first introduce ghost-independent states, which are states in which the ghost variables have been "erased".

Definition 7.3. Given a state $s$, the ghost-independent state $s^{\natural}$ is the state that interprets all the symbols in the same way as $s$, except ghost variables which are all interpreted by the constant function returning $\perp$.

An assertion $\varphi \in \mathcal{F}(\operatorname{Var})$ that contains no ghost variable holds in a state $s$ if and only if it holds in the state $s^{\natural}$.

Proof of Proposition 7.3. The proof addresses each statement of the proposition in turn.

Proof of (i). Consider a rule program $\mathcal{R}=\left\{r_{1}, \ldots, r_{n}\right\}$. The parallel program with while programs as processes derived from $\mathcal{R}$ is $W_{\mathcal{R}} \equiv\left[W_{r_{1}}\|\ldots\| W_{r_{n}}\right]$, with for $i=1, \ldots, n$ :

$$
\begin{aligned}
W_{r_{i}}\left(\vec{o}_{i}: \vec{T}_{i}\right) \equiv & \ell_{0}: \delta_{i}:=\text { false } \\
& \ell_{1}: \text { while } \neg \delta_{i} \text { begin } \\
& \left.\ell_{2}: \text { if } g_{i} \text { then } a_{i} ; \delta_{i}:=\text { true else skip } \text { i }\right\rangle \\
& \ell_{3}: \text { end }
\end{aligned}
$$

Let $s$ and $s^{\prime}$ be two $\mathcal{T}$-compliant states such that $s \stackrel{W}{\longrightarrow} s^{\prime}$, that is, there is a terminating execution of the parallel program $W_{\mathcal{R}}$ from $s$, that results in $s^{\prime}$. This means that there exist some states $s_{0}, \ldots, s_{m}$ such that

$$
s=s_{0} \underset{\ell^{1}}{\stackrel{w_{1}}{\longrightarrow}} s_{1} \frac{w_{2}}{\ell^{2}} \ldots \frac{w_{m-1}}{\ell^{m-1}} s_{m-1} \frac{w_{m}}{\ell^{m}} s_{m}=s^{\prime}
$$


For each $j=1, \ldots, m$, the transition from $s_{j-1}$ to $s_{j}$ involves a process instance $w_{j}=\left(W_{r_{i_{j}}}, \vec{O}_{j}\right)$, where $i_{j} \in\{1, \ldots, n\}$ is the index of a rule $r_{i_{j}} \in \mathcal{R}$, and $\vec{O}_{j} \in \mathcal{M}^{\left(r_{i_{j}}\right)}$ is a tuple of objects well-typed for the rule $r_{i_{j}}$; and a statement of the process $W_{r_{i_{j}}}$, labeled by $\ell^{j} \in\left\{\ell_{0}, \ell_{1}, \ell_{2}\right\}$. The transition occurs when the statement of $W_{r_{i_{j}}}$ is executed at $\ell^{j}$ on the objects $\vec{O}_{j}$, that is, under some valuation $\nu$ that maps the variables in $\vec{o}_{i_{j}}$ onto the objects in $\vec{O}_{j}$, and is hence well-typed for $\left(\vec{o}_{i_{j}}: \vec{T}_{i_{j}}\right)$.

Let us now consider the ghost-independent states $s_{0}^{\natural}, \ldots, s_{m}^{\natural}$ corresponding to the states of the transition sequence above, and let us examine for each $j=1, \ldots, m$, the relationship between $s_{j-1}^{\natural}$ and $s_{j}^{\natural}$, depending on the statement at $\ell^{j}$.

(a) If $\ell^{j}=\ell_{0}$, the executed statement relates only to the ghost variable $\delta_{i_{j}}$, so one has $s_{j-1}^{\natural}=s_{j}^{\natural}$. In addition one has $s_{j}, \nu \models \neg \delta_{i_{j}}$, which implies that the next transition for the process instance $w_{j}$ will execute the statement at $\ell_{1}$ as described by case (b) below.

(b) If $\ell^{j}=\ell_{1}$ and $s_{j-1}, \nu \models \neg \delta_{i_{j}}$, the next transition for the process instance $w_{j}$ will enter the loop at $\ell_{2}$, as described by cases (c) and (d). Also, since no action is performed in this transition, one has $s_{j-1}=s_{j}$ and hence $s_{j-1}^{\natural}=s_{j}^{\natural}$.

(c) If $\ell^{j}=\ell_{2}$ and $s_{j-1}, \nu \not \models g_{i_{j}}$, the statement executed is skip, so one has $s_{j-1}^{\natural}=s_{j}^{\natural}$. The next transition for the process instance $w_{j}$ will execute the statement at $\ell_{1}$ as described by case (b).

(d) If $\ell^{j}=\ell_{2}$ and $s_{j-1}, \nu \models g_{i_{j}}$ the statement executed in the transition is $a_{i_{j}} ; \delta_{i_{j}}:=$ true, meaning on the one hand that $\left\langle s_{j-1}^{\natural}, s_{j}^{\natural}\right\rangle, \nu \models \hat{a}_{i_{j}}$, and on the other hand that $s_{j}, \nu \models \delta_{i_{j}}$. The next transition for $w_{j}$ will therefore execute the statement at $\ell_{1}$ as described by case (e).

(e) If $\ell^{j}=\ell_{1}$ and $s_{j-1}, \nu \models \delta_{i_{j}}$ the next transition for the process instance $w_{j}$ will end its execution at $\ell_{3}$.

Since by hypothesis the execution of $W_{\mathcal{R}}$ from $s$ terminates, the last transition related to each process instance corresponds to the case (e) above. It follows from the case study that the preceding transition related to the same process instance must correspond to case (d). In this transition we have $s_{j-1}^{\natural}, \nu \models g_{i_{j}}$ and $\left\langle s_{j-1}^{\natural}, s_{j}^{\natural}\right\rangle, \nu \models \hat{a}_{i_{j}}$, that is, $s_{j-1}^{\natural} \stackrel{\left(r_{i_{j}}, \vec{O}_{j}\right)}{\longrightarrow} s_{j}^{\natural}$. In all other transitions related to this process instance we have $s_{j-1}^{\natural}=s_{j}^{\natural}$.

Since each well-typed rule instance that can be formed out of $\mathcal{R}$ and $\mathcal{M}$ occurs exactly once in the sequence, we have $s_{0}^{\natural} \stackrel{\mathcal{R}}{\longrightarrow} s_{m}^{\natural}$. As ghost variables are neither considered nor modified during the execution of $\mathcal{R}$, we have $s \stackrel{\mathcal{R}}{\longrightarrow} s^{\prime}$ as well. In other words, the execution of $W_{\mathcal{R}}$ is also an execution of $\mathcal{R}$.

Proof of (ii). We now prove the second statement of Proposition 7.3 by exhibiting a rule program whose derived parallel program with while programs as processes does not terminate. Consider again the rule program $\mathcal{R}=\left\{r_{1}, r_{2}\right\}$ below

$$
\begin{aligned}
& r_{1}(x: T): x \cdot a=1 \rightarrow x \cdot b:=1 \\
& r_{2}(x: T): x \cdot b=1 \rightarrow x \cdot c:=1
\end{aligned}
$$


The parallel program with while programs as processes derived from $\mathcal{R}$ is the program $W_{\mathcal{R}} \equiv\left[W_{r_{1}} \| W_{r_{2}}\right]$, with

$$
\begin{aligned}
W_{r_{1}}(x: T) \equiv & \delta_{1}:=\text { false; } \\
& \text { while } \neg \delta_{1}\left\langle\text { if } x . a=1 \text { then } x . b:=1 ; \delta_{1}:=\text { true else skip } \mathrm{fi}\right\rangle \\
W_{r_{2}}(x: T) \equiv & \delta_{2}:=\text { false; } \\
& \text { while } \neg \delta_{2}\left\langle\text { if } x . b=1 \text { then } x . c:=1 ; \delta_{2}:=\text { true else skip } \mathrm{fi}\right\rangle
\end{aligned}
$$

This program does not terminate when executed from the state $s_{0}$ defined as

$$
\begin{aligned}
\mathcal{M}(\mathrm{T}) & =\{\mathrm{X}\} \\
s_{0} & =a^{s_{0}}: \mathrm{X} \mapsto 0 \quad b^{s_{0}}: \mathrm{X} \mapsto 0 \quad c^{s_{0}}: \mathrm{X} \mapsto 0
\end{aligned}
$$

because none of the rule guards ever becomes valid.

\subsubsection{Rule Programs vs. Parallel Programs}

Given a rule program $\mathcal{R}$, the parallel program $W_{\mathcal{R}}$ derived from $\mathcal{R}$ as defined in the previous section fails to reproduce the behavior of $\mathcal{R}$ from the viewpoint of termination.

Is it possible to devise a systematic and compositional way of building a parallel program composed of while programs, possibly with ghost variables, out of a rule program, so that the parallel program and the rule program have the same behavior in the sense that they satisfy the same correctness formulas?

By "compositional", we mean that the parallel program has one process per rule; the variables and attribute symbols of each process are taken among those of the corresponding rule; the generation of the process is based only on the rule, and is thus independent from the other rules of the rule program. Again the answer is: no. That is, it is not possible to devise a way of generating a parallel program that simulates a rule program.

Theorem 7.4. There exists no function $\Pi$ that maps a rule $r$ onto a while program with ghost variables, where $\Pi(r) \in \mathcal{W}(\operatorname{var}(r)$, Ghost) and $\operatorname{Attr}(\Pi(r)) \subseteq$ $\operatorname{Attr}(r)$, such that given a rule program $\mathcal{R}=\left\{r_{1}, \ldots, r_{n}\right\}$, the parallel program $\left[\Pi\left(r_{1}\right)\|\ldots\| \Pi\left(r_{n}\right)\right]$ satisfies the same correctness formulas as $\mathcal{R}$, either in the sense of partial or of total correctness.

Proof. To prove the theorem, we exhibit a counterexample. Consider the three rule programs $\mathcal{R}_{1}=\left\{r_{1}\right\}, \mathcal{R}_{2}=\left\{r_{2}\right\}$ and $\mathcal{R}_{3}=\left\{r_{1}, r_{2}\right\}$ composed with the two rules below:

$$
\begin{aligned}
& r_{1}(x: T): x \cdot a=1 \rightarrow x \cdot a:=2 \\
& r_{2}(x: T): \text { true } \rightarrow \text { x.a }:=1
\end{aligned}
$$

These programs satisfy the following correctness formulas:

$$
\begin{aligned}
& \{\forall x: \mathrm{T}(x . a=0)\} \mathcal{R}_{1}\{\forall x: \mathrm{T}(x . a=0)\} \\
& \{\forall x: \mathrm{T}(x . a=0)\} \mathcal{R}_{2}\{\forall x: \mathrm{T}(x . a=1)\} \\
& \{\forall x: \mathrm{T}(x . a=0)\} \mathcal{R}_{3}\{\forall x: \mathrm{T}(x . a=2)\} .
\end{aligned}
$$

Let us assume that the function $\Pi$ described above exists. In addition, let us note $p \equiv \forall x: \mathrm{T}(x \cdot a=0)$. 
If $\Pi$ exists, then the parallel program $P_{1}=\left[\Pi\left(r_{1}\right)\right]$ has the same behavior as $\mathcal{R}_{1}$. Firstly, this implies that $P_{1}$ terminates when executed from a state that satisfies $p$ : otherwise it would satisfy in the sense of partial correctness any correctness formula with $p$ as its precondition, which $\mathcal{R}_{1}$ does not. Secondly, when $P_{1}$ is executed from a state that satisfies $p$, it results in a state where the same holds. In such an execution, the process instances executed are all the instances of $\Pi\left(r_{1}\right)$ on the instances of the type $\mathrm{T}$, that is, $I_{1}=\left\{\left(\Pi\left(r_{1}\right), X\right) \mid\right.$ $X \in \mathcal{M}(\mathrm{T})\}$.

Similarly, the parallel program $P_{2}=\left[\Pi\left(r_{2}\right)\right]$, when executed from a state that satisfies $p$, terminates and results in a state where the value of $a$ is 1 for all instances of the type T. The process instances executed are $I_{2}=\left\{\left(\Pi\left(r_{2}\right), X\right) \mid\right.$ $X \in \mathcal{M}(\mathrm{T})\}$.

Consider now the parallel program $P_{3}=\left[\Pi\left(r_{1}\right) \| \Pi\left(r_{2}\right)\right]$, and its executions from a state that satisfies $p$. The process instances to execute are $I_{1} \cup I_{2}$. Since the execution of $P_{1}$ from the same state terminates, there is an execution of $P_{3}$ in which all the process instances in $I_{1}$ are executed and terminate, before an instance of $\Pi\left(r_{2}\right)$ is executed. The state at this point of the execution still satisfies $p$, as seen above. In this execution of $P_{3}$, all the instances of $\Pi\left(r_{2}\right)$ are then executed and terminate.

The program $P_{3}$ thus terminates, but in a state where $\forall x: \mathrm{T}(x \cdot a=1)$. This contradicts the assumption that $P_{3}$ and $\mathcal{R}_{3}$ satisfy the same correctness formulas.

We have just shown with Theorem 7.4 that, in general, a rule program cannot be simulated by a parallel program whose processes are while programs possibly involving ghost variables under a few reasonable syntactic constraints. As a consequence, in general one cannot prove a correctness formula for a rule program by applying a proof method such as the Owicki-Gries one [OG76, AdBO09] to a parallel program built from the rule program.

This result is basically due to the fact that the execution of a rule instance can have an effect on the applicability of another rule instance, whereas it is not the case among process instances. In other words, the individual processes of a parallel program cannot simulate the global scheduling performed by the rule engine.

In particular, this result is not the consequence of the constraint for ghost variables to be local to each process instance. Assume indeed that we allow additional variables to be shared by all the processes of a parallel program (these additional variables could be introduced in our language as function symbols of arity 0). Assume also that there exists a function Init that maps the rules of a rule program onto some while program, such that the parallel program $P \equiv \operatorname{Init}\left(r_{1}, \ldots, r_{n}\right) ;\left[\Pi\left(r_{1}\right)\|\ldots\| \Pi\left(r_{n}\right)\right]$ satisfies the same correctness formulas as the rule program $\left\{r_{1}, \ldots, r_{n}\right\}$.

Consider the rule program $\mathcal{R}=\left\{r_{1}, r_{2}, r_{3}\right\}$ composed with the rules below:

$$
\begin{aligned}
& r_{1}(x: T): x \cdot a=1 \rightarrow x \cdot a:=2 \\
& r_{2}(x: T): \text { true } \rightarrow \text { x.a }:=1 \\
& r_{3}(x: T): x \cdot a=2 \rightarrow x \cdot a:=3
\end{aligned}
$$

Consider an execution of $\mathcal{R}$ on a working memory that contains only one object $\mathrm{X} \in \mathcal{M}(\mathrm{T})$. As noted in Section 6.1.1, in the final configuration of this execution, 
for each $i=1,2,3$ either the rule instance $\left(r_{i}, \mathrm{X}\right)$ is not eligible or the guard $g_{i}$ does not hold on $\mathrm{X}$. This is the condition that the parallel program $P$ has to encode as the exit condition of each process $\Pi\left(r_{i}\right)$.

Contrary to the eligibility of each rule instance, which can vary only as the consequence of the rule instance own application, the truth value of a rule guard on the object $\mathrm{X}$ can vary as the result of the application of another rule. For example, executing $\left(r_{2}, \mathrm{X}\right)$ in a state $s$ where $a^{s}(\mathrm{X})=0$ changes the truth value of $g_{1}$ on X. This change must be reflected by the update of a shared variable $\gamma_{1}$ in order to be taken into account by the exit conditions of all processes, including $\Pi\left(r_{3}\right)$. However, the shared variable can be updated only by the process $\Pi\left(r_{1}\right)$, since $\Pi$ is a function of each rule only. If the process $\Pi\left(r_{3}\right)$ is scheduled after $\Pi\left(r_{2}\right)$ has changed the truth value of $g_{1}$ but before $\Pi\left(r_{1}\right)$ has updated $\gamma_{1}$, then $\Pi\left(r_{3}\right)$ will base its execution on a wrong value of $\gamma_{1}$, possibly exiting too early.

\subsection{Correctness of Nondeterministic Programs}

Unlike parallel programs, a nondeterministic program based on Dijkstra's dood statement can be derived from a given rule program and satisfy the same correctness formulas. However, the proof rule for do-od is not compositional but instead involves a global invariant.

Nondeterministic programs are another programming paradigm of declarative nature. The central construct of nondeterministic programs is the guarded command introduced by Edsger Dijkstra [Dij75]. The implicit aspect of nondeterministic programs lies in the unspecified choice between several valid guards in nondeterministic statements.

In this section, we first briefly introduce nondeterministic programs, so as to connect their standard definition to our formal framework. Then we compare rule programs and nondeterministic programs, to find that there is a way of deriving a nondeterministic program with ghost variables from a rule program, so that both have the same behavior.

\subsubsection{Nondeterministic Programs}

\section{Syntax}

As just mentioned, the central construct of nondeterministic programs is the guarded command. A guarded command is made of a Boolean expression (the guard) and a program (the command). It does not constitute a statement in itself, but serves as a building block for the nondeterministic conditional and loop. As we will only use the nondeterministic loop, we do not include the nondeterministic conditional in our language of nondeterministic programs.

In Fig. 7.5, we define the grammar of the $\mathcal{N}(V, G)$ language of nondeterministic programs, which resembles the language of while programs, with the do-od nondeterministic loop in lieu of the while loop. Note that, to avoid any confusion with logical implication or with the concrete syntax of rules, we use a special arrow $\rightarrow$ between the guard and the command in guarded commands.

The variables that occur in a nondeterministic program usually come with associated types, for example because they were inherited from a rule program. 


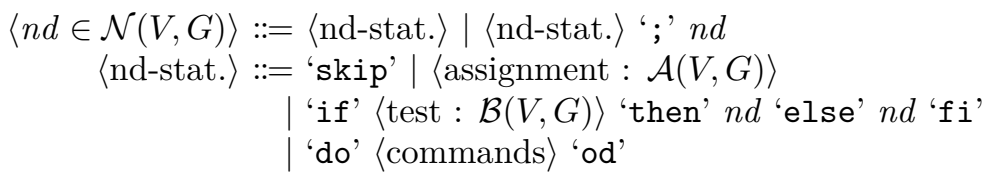

Figure 7.5: Nondeterministic programs.

As for the other languages that we introduced in this chapter, we define that a nondeterministic program is well-typed for a type constraint by induction on its structure, based on well-typed formulas and assignment.

\section{Saturated semantics}

The standard semantics for executing a nondeterministic loop on some input data would be to consider the guards that hold in the current state, to pick one nondeterministically, to update the current state by executing the corresponding command, and to repeat this process until no guard holds [Dij75].

Example. Assume that, for some reason, one wants to reduce the ages of two persons to their greatest common divisor. This could be achieved by the following nondeterministic program under the standard semantics for do-od loops:

$$
\begin{array}{r}
\mathrm{P}(\mathrm{p}: \text { Person, } \mathrm{q}: \text { Person }): \text { do } \mathrm{p} . \text { age }>\mathrm{q} . \text { age } \rightarrow \text { p.age }: \\
\text { p.age }<\mathrm{q} \text {.age }-\mathrm{q} \cdot \text { age } \\
\text { q.age }:=\mathrm{q} . \text { age }- \text { p.age od }
\end{array}
$$

For example, consider an execution of $P$ from the state $s_{0}$ defined below, on some input data (that is, under some valuation) that maps $p$ onto Alice and $q$ onto Bob:

$$
a g e^{s_{0}}(\text { Alice })=24 \quad a g e^{s_{0}}(\mathrm{Bob})=18 .
$$

In the state $s_{0}$, the first guard holds, and the execution of the first command results in the state $s_{1}$ where $a g e^{s_{1}}$ (Alice) $=6$. In $s_{1}$, the second guard holds, leading to $s_{2}$ where $a g e^{s_{2}}(\mathrm{Bob})=12$. There again, the second guard holds, leading to $s_{3}$ where $a g e^{s_{3}}($ Alice $)=a g e^{s_{3}}(\mathrm{Bob})=6$. The loop and the program then terminate.

As with parallel programs (see Section 7.1.1) we are going to extend the standard semantics of nondeterministic programs by adapting the instantiation of programs to support executions on a working memory.

A nondeterministic loop is of the form do $g c_{1}[\ldots] g c_{n}$ od, where for $i=1, \ldots, n$ each $g c_{i} \equiv g_{i} \longrightarrow c_{i}$ is a guarded command with $g_{i} \in \mathcal{B}$ (Var, Ghost) and $c_{i} \in \mathcal{N}$ (Var, Ghost). For each $i=1, \ldots, n$, the guarded command $g c_{i}$ involves variables in its guard and command, which come with a type constraint $\left(\vec{o}_{i}: \vec{T}_{i}\right)$ for them.

When executing a nondeterministic loop on a working memory $\mathcal{M}$, the saturated semantics considers for a guarded command $g c_{i}$ all the instances of the type $T_{j}^{i}$ as possible values for each variable $o_{j}^{i}$ that occurs in the guarded command. As a consequence, as many instances of each guard $g_{i}$ are assessed, and all of them that hold in the current state are candidate to be picked. 
Example. Consider the following nondeterministic program:

$\mathrm{P}(\mathrm{p}:$ Person, $\mathrm{q}:$ Person $):$ do $\mathrm{p}$. age $>\mathrm{q}$. age $\rightarrow$ p.age $:=$ p.age $-\mathrm{q}$.age od

and its execution under the saturated semantics on a working memory such as $\mathcal{M}($ Person $)=\{$ Alice, Bob $\}$.

In this execution, four instances of the guarded command are created, corresponding to the four ways of mapping the two variables $p$ and $q$ onto the two instances of the type Person. The guard p.age > q.age is thus assessed in each successive state of the execution under the following four valuations:

$$
\begin{array}{lll}
\nu_{1}: & p \mapsto \text { Alice } & q \mapsto \text { Alice } \\
\nu_{2}: & p \mapsto \text { Alice } & q \mapsto \text { Bob } \\
\nu_{3}: & p \mapsto \text { Bob } & q \mapsto \text { Alice } \\
\nu_{4}: & p \mapsto \text { Bob } & q \mapsto \text { Bob. }
\end{array}
$$

Regardless of the state, the guard will never hold under $\nu_{1}$ nor under $\nu_{4}$. Therefore, we end up with the same guards being assessed in each successive state, and the same commands executed, as in the previous example.

Note that with this same program and the saturated semantics, we can compute the greatest common divisor of the ages of any number of persons by simply adding them to the working memory.

\subsubsection{Nondeterministic Program Derived from a Rule Pro- gram}

Definition 7.4. Given a rule program $\mathcal{R}=\left\{r_{1}, \ldots, r_{n}\right\}$, the nondeterministic program derived from $\mathcal{R}$ is the program defined as follows, with $r_{i}=$ $\left(\vec{o}_{i}, \vec{T}_{i}, g_{i}, a_{i}\right)$ and $\delta_{i} \in$ Ghost for $i=1, \ldots, n$ :

$$
P_{\mathcal{R}}(\vec{o}: \vec{T}) \stackrel{\text { def }}{\equiv} \delta_{1}:=\text { false } ; \ldots ; \delta_{n}:=\text { false } ; \text { do } \square_{i=1}^{n} g_{i} \wedge \neg \delta_{i} \neg a_{i} ; \delta_{i}:=\text { true od }
$$

The notations $\vec{o}$ and $\vec{T}$ denote the respective concatenations of the tuples $\vec{o}_{i}$ and $\vec{T}_{i}$ for $i=1, \ldots, n$.

As for parallel programs in Section 7.2.4, for each rule $r_{i}$ we use a ghost variable $\delta_{i}$ (with $\delta$ standing for "done") with Boolean values to encode the one-shot eligibility strategy.

Example. Consider the rule program $\mathcal{R}=\left\{r_{1}, r_{2}\right\}$ below:

$$
\begin{aligned}
& \mathrm{r}_{1}(\mathrm{x}: \mathrm{P}): \mathrm{x} . \text { age } \leq 12 \rightarrow \mathrm{x} . \text { status }:=\text { Child } \\
& \mathrm{r}_{2}(\mathrm{y}: \mathrm{P}): \mathrm{y} . \text { status }=\text { Child } \rightarrow \text { y.gift }:=\text { TeddyBear }
\end{aligned}
$$

The nondeterministic program $P_{\mathcal{R}}$ derived from $\mathcal{R}$ is:

$$
\begin{aligned}
\mathrm{P}_{\mathcal{R}}(\mathrm{x}: \mathrm{P}, \mathrm{y}: \mathrm{P}): & \delta_{1}:=\text { false } ; \delta_{2}:=\text { false } \\
& \text { do x.age } \leq 12 \& \neg \delta_{1} \neg \mathrm{x} . \text { status }:=\text { Child } ; \delta_{1}:=\text { true } \llbracket \\
& \text { y.status }=\text { Child \& } \neg \delta_{2} \neg \text { y.gift }:=\text { Bear } ; \delta_{2}:=\text { true od }
\end{aligned}
$$


The nondeterministic program derived from a given rule program can be seen as the partial evaluation on the rule program of the description given in Chapter 5 of a rule engine under the one-shot execution strategy. We show below that both a rule program $\mathcal{R}$ and the nondeterministic program $P_{\mathcal{R}}$ have the same behavior.

Theorem 7.5. The nondeterministic program $P_{\mathcal{R}}$ derived from a given rule program $\mathcal{R}$ satisfies the same correctness formulas as $\mathcal{R}$ in the sense of total correctness.

Since $\mathcal{R}$ always terminates under the one-shot execution strategy, the indication "in the sense of total correctness" implies that $P_{\mathcal{R}}$ always terminates as well.

Proof. Consider a rule program $\mathcal{R}=\left\{r_{1}, \ldots, r_{n}\right\}$ with $r_{i}=\left(\vec{o}_{i}, \vec{T}_{i}, g_{i}, a_{i}\right)$ for $i=1, \ldots, n$, and the nondeterministic program $P_{\mathcal{R}}$ derived from it. To prove the theorem, we have to show that $P_{\mathcal{R}}$ always terminates, that any execution of $P_{\mathcal{R}}$ is an execution of $\mathcal{R}$, and conversely that any execution of $\mathcal{R}$ is an execution of $P_{\mathcal{R}}$.

Termination of $P_{\mathcal{R}}$. When the nondeterministic loop in $P_{\mathcal{R}}$ is first executed, all ghost variables $\delta_{i}$ are false for all object tuples. Either no rule guard holds, and then $P_{\mathcal{R}}$ terminates immediately; or at least one rule guard holds, and the execution of the picked command sets the corresponding ghost variable to true. In other words, on each loop iteration, either the program ends or one $\delta_{i}$ is set to true among those that were false. It follows that the loop eventually ends, and hence that $P_{\mathcal{R}}$ terminates.

Any execution of $P_{\mathcal{R}}$ is an execution of $\mathcal{R}$. Consider two $\mathcal{T}$-compliant states $s$ and $s^{\prime}$ such that $s \stackrel{P_{\mathcal{R}}}{\longrightarrow} s^{\prime}$, that is, there is a (terminating) execution of $P_{\mathcal{R}}$ from $s$ that results in $s^{\prime}$. This means that there exist some states $s_{0}, \ldots, s_{m}$ such that

$$
s \stackrel{\delta_{1}:=\text { false } ; \ldots ; \delta_{n}:=\text { false }}{\longrightarrow} s_{0} \stackrel{G C_{1}}{\longrightarrow} s_{1} \stackrel{G C_{2}}{\longrightarrow} \ldots \stackrel{G C_{m-1}}{\longrightarrow} s_{m-1} \stackrel{G C_{m}}{\longrightarrow} s_{m}=s^{\prime}
$$

where for $j=1, \ldots, m, G C_{j}=\left(g c_{i_{j}}, \vec{O}_{j}\right)$ is an instance of the guarded command corresponding to the rule $r_{i_{j}}$ on a tuple of objects well-typed for this rule.

For each $j=1, \ldots, m$, the transition $s_{j-1} \stackrel{G C_{j}}{\longrightarrow} s_{j}$ occurs because the guard $g_{i_{j}}$ holds in the state $s_{j-1}$ under some valuation $\nu$ that maps the variables $\vec{o}_{i_{j}}$ onto the objects $\vec{O}_{j}$, and because the ghost variable $\delta_{i_{j}}$ is false in the state $s_{j-1}$ on the objects $\vec{O}_{j}$. The state $s_{j}$ results from the execution of the action $a_{i_{j}}$ in $s_{j-1}$ under $\nu$ and then from the update of the ghost variable $\delta_{i_{j}}$ on the objects $\vec{O}_{j}$. In particular, we have

$$
s_{j-1}, \nu \models g_{i_{j}} \quad \text { and } \quad\left\langle s_{j-1}, s_{j}\right\rangle, \nu \models \hat{a}_{i_{j}} .
$$

On the other hand, let us define for each $j=1, \ldots, m$ the set

$$
E_{j}=\left\{\left(r_{i}, \vec{O}\right) \mid 1 \leq i \leq n, \vec{O} \in \mathcal{M}^{\left(r_{i}\right)}, \delta_{i}^{s_{j}}(\vec{O})=\text { false }\right\}
$$

of all the instances of rules of $\mathcal{R}$ whose corresponding ghost variable is false on the objects of the instance. In each transition $s_{j-1} \stackrel{G C_{j}}{\longrightarrow} s_{j}$ described above, we have

$$
\left(r_{i_{j}}, \vec{O}_{j}\right) \in E_{j-1} \quad \text { and } \quad\left(r_{i_{j}}, \vec{O}_{j}\right) \notin E_{j} .
$$


The assertions (a) and (b) mean that $\left\langle E_{j}, s_{j}\right\rangle$ is a configuration that results from a transition from $\left\langle E_{j-1}, s_{j-1}\right\rangle$ by the application of the rule instance $\left(r_{i_{j}}, \vec{O}_{j}\right)$ under the one-shot eligibility strategy.

As $E_{0}=\mathrm{RI}_{s_{0}}(\mathcal{R})$, it follows that $s_{0} \stackrel{\mathcal{R}}{\longrightarrow} s_{m}$, that is, there is an execution of the rule program $\mathcal{R}$ from the state $s_{0}$, that results in $s_{m}$. Since $s^{\natural}=s_{0}^{\natural}$ and $s_{m}=s^{\prime}$, we have $s \stackrel{\mathcal{R}}{\longrightarrow} s^{\prime}$, and hence any execution of $P_{\mathcal{R}}$ is an execution of $\mathcal{R}$.

Any execution of $\mathcal{R}$ is an execution of $P_{\mathcal{R}}$. A reasoning similar to the previous, symmetric one gives this result. This ends the proof of Theorem 7.5.

As a conclusion, we now know how to get a nondeterministic program that satisfies the same correctness formulas in the sense of total correctness as any rule program considered for execution under the one-shot strategy. This means that given a correctness formula $\{p\} \mathcal{R}\{q\}$ for a rule program $\mathcal{R}$, we could use proof rule (DO-OD) below [Apt84] on the nondeterministic program derived from $\mathcal{R}$ to prove it.

$$
\begin{gathered}
\frac{\left\{I \wedge B_{i}\right\} S_{i}\{I\} \quad \text { for } i=1, \ldots, n}{\{I\} \text { do } \prod_{i=1}^{n} B_{i} \neg S_{i} \text { od }\left\{I \wedge \bigwedge_{i=1}^{n} \forall \vec{o}_{i}: \vec{T}_{i} \neg B_{i}\right\}} \\
\text { where } I \in \mathcal{F}(\emptyset, \text { Ghost })
\end{gathered}
$$

However this method is not compositional, in that it relies on a global invariant and not on individual correctness formulas for the rules of $\mathcal{R}$. This is a drawback in our context, as it does not leverage the declarative nature of rule programs and the modularity of rules.

Finding such a compositional proof rule is the subject of the next part. 


\section{Part III}

\section{Proof Rules for Rule Programs}





\section{Chapter 8}

\section{Main Steps in Rule Program Verification}

The purpose of the present Part III is to propose a compositional verification method for rule programs. This verification method is based on proof rules to derive a correctness formula for a rule program, from individual correctness formulas for the rules of the program. It is compositional in the sense that it exploits the modular, declarative nature of rule programs, by deriving a global piece of information on the rule program from local knowledge about each individual rule.

In the present chapter, we explain the foundations of our verification method with a proof rule for a simple, although not trivial, class of rule programs. The next chapter exposes our verification method for general rule programs, based on a sound and relatively complete proof rule. Chapter 10 explores other classes of rule programs and provides the proof rules suited to each case.

We still work within the setting exposed in Section 6.1, that is, rule programs are executed under the one-shot execution strategy and we use the classical definitions of proofs and proof rules, as reminded in that section.

The objective of a compositional verification method for rule programs is to help prove a correctness formula for a rule program by leveraging the declarative, modular structure of the program as a set of rules. Consider a rule program $\mathcal{R}=\left\{r_{1}, \ldots, r_{n}\right\}$ and a correctness formula $\{p\} \mathcal{R}\{q\}$ for this rule program. The verification method should allow us to prove this correctness formula by proving "smaller" correctness formulas $\left\{p_{i}\right\} r_{i}\left\{q_{i}\right\}$ for the individual rules in the program. Indeed, proving the correctness formulas for the rules is considered simpler, because they involve only the objects handled in one execution of one rule.

However, we have seen with Proposition 6.2 that correctness formulas do not trivially transpose from rules to rule programs. This is due to the possible interference cases between the multiple rule instance applications in a rule program execution and the pre- and postconditions of the correctness formulas.

Interference between the statements of a program and the individual correctness formulas for these statements is a well-known phenomenon in parallel programs [Lip75, OG76, Lam77]. Tackling them is the focus of verification methods for parallel programs, such as the one proposed by Owicki and Gries 
[OG76]. This approach is exposed in great detail in [AO97, AdBO09], with a progressive introduction of gradually more complex classes of parallel programs.

Although Theorem 7.4 has invalidated the idea of mapping rule programs onto parallel ones to use a verification method devised for parallel programs, our approach to addressing interference in rule programs follows similar paths. The specificities come from the kinds of interference that can occur during the execution of a rule program.

\subsection{Interference Cases in Rule Programs}

Two kinds of interference can occur during the execution of a rule programs. Cross-rule interference is when the execution of a rule invalidates the pre- or postcondition of a correctness formula focused on another rule. Interference due to aliasing is when several rule instances refer to the same object, and this causes a pre- or postcondition to become invalid on this object.

An execution of a rule program is a sequence of rule instance applications. Each rule instance application has an effect on the current state. Those effects that are called interference cases are the ones that prevent the derivation from the correctness formulas focused on the individual rules to the global correctness formula to prove. An interference has the undesired effect of leading to a state where the pre- or postcondition of an individual correctness formula no longer holds.

An interference thus involves the application of a rule instance and an assertion. In a rule program execution, two kinds of interference can occur: cross-rule interference and interference due to aliasing.

\subsubsection{Cross-Rule Interference}

Consider the two rules below:

$$
\begin{aligned}
& r_{1}(p: P): \text { p.age }>60 \rightarrow \text { p.bonus }:=\text { p.bonus }+10 \\
& r_{2}(p: P): \text { p.cat }=\text { Gold } \rightarrow \text { p.bonus }:=\text { p.bonus }+15
\end{aligned}
$$

The following correctness formulas hold for each of the individual rules $r_{1}$ and $r_{2}$ :

$$
\begin{gathered}
\{\text { true }\} r_{1}\{\text { true }\} \\
\{p . \text { bonus }=0 \wedge \text { p.cat }=\text { Gold }\} r_{2}\{\text { p.bonus }=15\} .
\end{gathered}
$$

The interference between the rules $r_{1}$ and $r_{2}$ and these individual correctness formulas is visible when considering the following global correctness formula for the rule program $\mathcal{R}_{1}=\left\{r_{1}, r_{2}\right\}$ :

$$
\{\forall p: \mathrm{P}(\text { p.bonus }=0 \wedge \text { p.cat }=\mathrm{Gold})\} \mathcal{R}_{1}\{\forall p: \mathrm{P}(\text { p.bonus }=15)\} .
$$

This correctness formula is obtained by conjunction of the pre- and postconditions of the individual correctness formulas above, but it does not hold. Indeed, when executing the program $\mathcal{R}_{1}$ from a state where an instance of $\mathrm{P}$ both has an age greater than 60 and a Gold category, its bonus is increased by 25 . 
The interference here results from the fact that neither the pre- nor the postcondition of the correctness formula for $r_{2}$ is guaranteed to be preserved by the application of $r_{1}$. This is why we call it cross-rule interference. In contrast, the same global correctness formula holds for the rule program $\mathcal{R}_{2}=\left\{r_{2}\right\}$, which does not contain $r_{1}$ :

$$
\{\forall p: \mathrm{P}(\text { p.bonus }=0 \wedge \text { p.cat }=\mathrm{Gold})\} \mathcal{R}_{2}\{\forall p: \mathrm{P}(\text { p.bonus }=15)\} .
$$

Furthermore, the following correctness formulas for $\mathcal{R}_{1}$ hold, and can be derived by the proof rules given in Section 8.3 or in Chapter 9 from individual correctness formulas that do not suffer from cross-rule interference with the rules in the program:

$$
\begin{aligned}
& \begin{array}{l}
\{\forall p: \mathrm{P}(\text { p.bonus }=0 \wedge \text { p.cat }=\mathrm{Gold} \wedge \text { p.age } \leq 60)\} \\
\{\forall p: \mathrm{P}(\text { p.bonus }=15)\}
\end{array} \\
& \begin{array}{r}
\{\forall p: \mathrm{P}(\text { p.bonus }=0 \wedge p . \text { cat }=\mathrm{Gold})\} \\
\{\forall p: \mathrm{P}(\text { p.bonus }=15 \vee p . \text { bonus }=25)\}
\end{array} \\
& \{\forall p: \mathrm{P}(\text { p.bonus }=0 \wedge \text { p.cat }=\mathrm{Gold})\} \mathcal{R}_{1} \\
& \{\forall p: \mathrm{P}(\text { p.age } \leq 60 \wedge \text { p.bonus }=15 \vee \text { p.age }>60 \wedge \text { p.bonus }=25)\} .
\end{aligned}
$$

\subsubsection{Interference due to Aliasing}

The aliasing phenomenon is inherited by rule programs from their use of objects. It names the fact that two variables can refer to the same object. As a consequence, an update of the object held in one variable can cause a change in the object held by another one. Aliasing is widely studied, for example by Morris [Mor82] from whom we adopt the approach, which consists in including a conditional expression in the formula that defines the effect of assignment. See Sections 4.4 and 5.3 for our handling of aliasing, and [AdBO09, p. 240] for additional references.

The two rules below provide an example of interference due to aliasing. They state the rules for assigning points on a discount card depending on the bonus of a person.

$$
\begin{aligned}
& \mathrm{r}_{3}(\mathrm{c}: \mathrm{C}, \mathrm{p}: \mathrm{P}): \text { p.bonus }<20 \rightarrow \text { c.points }:=\mathrm{c} \cdot \text { points }+5 \\
& \mathrm{r}_{4}(\mathrm{c}: \mathrm{C}, \mathrm{p}: \mathrm{P}): \text { p.bonus } \geq 20 \rightarrow \text { c.points }:=\text { c.points }+10
\end{aligned}
$$

These rules satisfy the following correctness formulas:

$$
\begin{aligned}
& \{\text { c.points }=0\} r_{3}\{\text { c.points }=0 \vee c \text {.points }=5\} \\
& \{\text { c.points }=0\} r_{4}\{\text { c.points }=0 \vee c \text { points }=10\} .
\end{aligned}
$$

The global correctness formula obtained by conjunction of the pre- and postconditions of these individual correctness formulas does obviously not hold for the rule program $\mathcal{R}_{3}=\left\{r_{3}, r_{4}\right\}$ :

$$
\{\forall c: \mathrm{C}(\text { c.points }=0)\} \mathcal{R}_{3}\{\forall c: \mathrm{C}(\text { c.points }=0)\} .
$$

Even the following correctness formulas, suggested by the ones that hold for $\mathcal{R}_{1}$ above, do not hold:

$$
\begin{aligned}
& \{\forall c: \mathrm{C}(\text { c.points }=0)\} \mathcal{R}_{3}\{\forall c: \mathrm{C}(\text { c.points }=0 \vee c . p o i n t s=5 \vee c . p o i n t s=10)\} \\
& \{\forall p: \mathrm{P} \forall c: \mathrm{C}(\text { p.bonus }<20 \wedge c . \text { points }=0)\} \mathcal{R}_{3}\{\forall c: \mathrm{C}(\text { c.points }=5)\} .
\end{aligned}
$$


Indeed, when $\mathcal{R}_{3}$ is executed from a state where three persons with bonuses lower that 20 share the same empty discount card, it results in a state where the discount card has 15 points. The interference here results from the fact that a given discount card can be referred to by several rule instances in an execution of $\mathcal{R}_{3}$.

Another illustration of such an interference due to aliasing is provided by the counterexample used in the proof of Proposition 6.2 on page 84. The rule program in this counterexample contains only one rule; interference occurs between several instances of this rule.

The rule program $\mathcal{R}_{3}$ could be modified to avoiding aliasing, by adding a binding between $p$ and $c$ to the guard of each rule:

$$
\begin{aligned}
& \mathrm{r}_{3}^{\prime}(\mathrm{c}: \mathrm{C}, \mathrm{p}: \mathrm{P}): \text { p.bonus }<20 \& \mathrm{p}=\mathrm{c} . \text { owner } \rightarrow \text { c.points }:=\mathrm{c} \text {.points }+5 \\
& \mathrm{r}_{4}^{\prime}(\mathrm{c}: \mathrm{C}, \mathrm{p}: \mathrm{P}): \text { p.bonus } \geq 20 \& \mathrm{p}=\mathrm{c} . \text { owner } \rightarrow \text { c.points }:=\mathrm{c} \text {.points }+10
\end{aligned}
$$

However these modified rules have a different behavior from the rules in $\mathcal{R}_{3}$. In other words, aliasing might be considered as a feature by the author of a rule program, who consciously leverages it to implement a given specification.

For example, when executed on a working memory that contains one instance of $\mathrm{P}$ for each person in a family, and one instance of $\mathrm{C}$ representing the discount card of the family, program $\mathcal{R}_{3}$ computes the discount points earned by the family. The following global correctness formula for $\mathcal{R}_{3}$ can then be derived, using the proof rule given in Chapter 9, from individual correctness formulas that do not suffer from interference due to aliasing:

$$
\{\forall c: \mathrm{C}(\text { c.points }=0)\} \mathcal{R}_{3}\{\forall c: \mathrm{C}(\text { c.points } \geq 0)\} .
$$

\subsection{Unary Rules}

Unary rules are rules with only one rule variable. Programs made of unary rules have the interesting property that no aliasing can occur during their executions. This eliminates one kind of interference, and offers a simplified setting to present the verification method in the rest of this chapter.

We introduce now unary rules, which provide a simplified setting where the core principles of our verification method can be more easily grasped. As we see below, the main contribution of unary rules is to eliminate interference due to aliasing.

Definition 8.1. A rule is a unary rule if it has at most one rule variable. An assertion is a unary assertion if it has at most one free variable.

Example. The following rules are unary:

$$
\begin{aligned}
& r_{1}(p: \text { Person }): p \cdot \text { age }>60 \rightarrow \text { p.bonus }:=p \cdot \text { bonus }+10 \\
& r_{2}(p: \text { Person }): \text { p.cat }=\text { Gold } \rightarrow \text { p.bonus }:=\text { p.bonus }+15
\end{aligned}
$$

The following assertions are unary:

$$
\begin{gathered}
\text { p.bonus }=0 \\
\text { true. }
\end{gathered}
$$


In contrast, the following rule and assertion are not unary:

$$
\begin{gathered}
\mathrm{r}_{3}(\mathrm{p}: \text { Person, } \mathrm{c}: \text { Cart }): \text { c.value }>500 \rightarrow \text { p.bonus }:=\text { p.bonus }+ \text { c.value } / 10 \\
\text { p.bonus } \leq \text { c.value. }
\end{gathered}
$$

By definition (see page 39), an assertion focused on a unary rule is a unary assertion.

The interest of unary rules comes from the constraint imposed by the grammar of expressions and discussed in Section 3.3.3, that formulas must be flat. The consequence is that neither the guard of a rule nor its action can contain a chain of attribute references, such as p.spouse.bonus. Combine this constraint with the one for rules, and hence for their guards and actions, to involve only one variable: the execution of a unary rule is affected by, and affects, only the object to which it is applied. In other words, unary rules and assertions cannot cause, or suffer from, interference due to aliasing. On the other hand however, not all rule programs can be expressed with unary rules, as illustrated by the program used as an example in Section 8.1.2.

Lemma 8.1 below formalizes the immunity of unary rules to aliasing. Before exposing it, we take the opportunity of simplifying notations for rule programs made of unary rules.

If a rule $r=(\vec{o}, \vec{T}, g, a)$ is unary, its tuple of variables $\vec{o}$ is actually a singleton. The sole variable of $r$ is then noted $o$ and its type is noted $T$; the rule is thus noted $r=(o, T, g, a)$. The rule guard $g$ is a unary assertion in $o$ and the rule action $a$ is an assignment to an attribute of $o$.

Given a rule program $\mathcal{R}=\left\{r_{1}, \ldots, r_{n}\right\}$ made of unary rules, we note each rule $r_{i}$ of the rule program $r_{i}=\left(o_{i}, T_{i}, g_{i}, a_{i}\right)$ with $a_{i} \equiv o_{i} \cdot f_{r_{i}}:=e_{r_{i}}$. We use the notation $\forall \vec{o}: \vec{T}$ as an abbreviation for $\forall o_{1}: T_{1} \ldots \forall o_{n}: T_{n}$.

Finally we simplify in a similar way the notations introduced in Sections 3.5.1 and 4.2.3 when renaming the variables of a rule or of an assertion focused on a rule. If $x$ is a variable, $r=(o, T, g, a)$ is a unary rule, and $\varphi$ is an assertion focused on $r$ (such as the guard $g$ of $r$, or the pre- or postcondition of a correctness formula focused on $r$ ), we note $r(x)$ and $\varphi(x)$ the rule $r$ and the assertion $\varphi$ where the variable $x$ has been substituted for $o$

Lemma 8.1. Given a unary rule $r=(o, T, g, a)$ and a unary assertion $\varphi \in$ $\mathcal{F}(\{x\})$ in some variable $x$, the rule $r$ preserves $\varphi$ under the condition $x \neq o$. That is,

$$
\{x \neq o \wedge \varphi\} r\{\varphi\}
$$

Proof. Consider two $\mathcal{T}$-compliant states $s$ and $s^{\prime}$, and a valuation $\nu$ well-typed for $(o: T)$, such that $s, \nu \models x \neq o \wedge \varphi$ and $s \stackrel{(r, \nu)}{\longrightarrow} s^{\prime}$.

If the rule instance $(r, \nu)$ is not applicable in $s$, then $s^{\prime}=s$ and hence $s^{\prime}, \nu \models \varphi$.

If the rule instance $(r, \nu)$ is applicable in $s$, then $\left\langle s, s^{\prime}\right\rangle, \nu \models \hat{a}$. The transition assertion $\hat{a}$ for the action $a \equiv o . f_{r}:=e_{r}$ is defined in Section 4.5 ; in particular and as noticed in Section 5.3.3, it follows from $x \neq o$ that

$$
\left\langle s, s^{\prime}\right\rangle, \nu \models x . f_{r}^{\prime}=x . f_{r} \wedge \bigwedge_{f \in \operatorname{Attr} \backslash\left\{f_{r}\right\}} x . f^{\prime}=x . f .
$$


That is, the states $s$ and $s^{\prime}$ give the same values to all attributes of the object onto which $\nu$ maps $x$. Since $\nu$ is arbitrary and $\varphi$ is unary in $x$, its truth value is the same in $s$ and $s^{\prime}$.

In all cases we thus have $s^{\prime}, \nu \models \varphi$, which proves the lemma.

\subsection{A First Proof Rule}

We propose in this section a reasonably simple proof rule suited for programs made of unary rules. We show that this proof rule is sound, by using the fact that at each step in the execution of a rule program, only one correctness formula focused on a rule makes a transition from its precondition to its postcondition.

Consider a rule program $\mathcal{R}$ made of unary rules. Consider also a collection of correctness formulas focused on the rules in $\mathcal{R}$. As just discussed, these rules and correctness formulas cannot suffer from interference due to aliasing. In the present section we give a proof rule that tackles the other source of interference, namely cross-rule interference. Both its form and the proof of its soundness will be reminiscent of the Owicki-Gries proof rule for parallel programs [OG76, AdBO09]. As noted in Section 7.2 though, the simulation of $\mathcal{R}$ by a parallel program, which would open the door to applying the OwickiGries proof rule, is not possible in general.

Theorem 8.2. Let $\mathcal{R}=\left\{r_{1}, \ldots, r_{n}\right\}$ be a rule program, and for $i=1, \ldots, n$ let $p_{i}, q_{i} \in \mathcal{F}\left(r_{i}\right)$ be assertions focused on the rules. If $\mathcal{R}$ is made of unary rules, then the proof rule below is sound:

$$
\begin{gathered}
\left\{p_{i}\right\} r_{i}\left\{q_{i}\right\} \quad \text { for } i=1, \ldots, n \\
\left.\left\{p_{i} \wedge p_{j}\right\} r_{j}\left\{p_{i}\right\}\right\} \text { for } i, j=1, \ldots, n \text { with } i \neq j \\
\left\{q_{i} \wedge p_{j}\right\} r_{j}\left\{q_{i}\right\}
\end{gathered}
$$

In addition to the correctness formulas focused on the rules, the premises of this proof rule contain correctness formulas expressing interference freedom, that is, the fact that no cross-rule interference can occur. Although it might not be obvious at first sight, these correctness formulas are not focused on a rule, as they involve both the variable $o_{i}$ brought by the assertion $p_{i}$ or $q_{i}$, and the variable $o_{j}$ brought by the assertion $p_{j}$ and the rule $r_{j}$. The semantics of such correctness formulas for single rules, which include variables that are not variables of the rule in their pre- and postconditions, has been discussed in Section 6.2.

The application of this proof rule is discussed in detail on an example in Section 8.4. Lemma 8.4 introduced in that section addresses the fact that the interference freedom correctness formulas are not focused on a rule.

As we show in Section 8.5, this proof rule is not complete, in the sense that there exist rule programs and global correctness formulas for these rule programs that cannot be proven with the proof rule. As for parallel programs, 
completeness is achieved with the introduction of ghost variables. This is exposed in Section 10.1 for the particular case of unary rules, and in Chapter 9 for the general case of rules of arbitrary arity.

The proof of Theorem 8.2 relies on the fact that, among the correctness formulas focused on the rules, only one makes a transition from its pre- to its postcondition at each step in an execution of the rule program; and that this correctness formula is the one related to the rule instance that is executed. We formalize this property in Lemma 8.3 below. To this end, we first define what it means for a configuration of the abstract machine used for execution of rule programs (see Section 5.4.1) to be consistent with a collection of correctness formulas focused on rules. The lemma is then used to prove the theorem.

Definition 8.2. Given a rule program $\mathcal{R}=\left\{r_{1}, \ldots, r_{n}\right\}$ made of unary rules, and correctness formulas $\left\{p_{i}\right\} r_{i}\left\{q_{i}\right\}$ for $i=1, \ldots, n$ focused on the rules of $\mathcal{R}$, a configuration $\langle E, s\rangle$ of the abstract machine used for execution of rule programs is consistent with the unary correctness formulas, or unary cf-consistent, if

for each $i \in\{1, \ldots, n\}$ and for any valuation $\nu$ well-typed for $\left(o_{i}: T_{i}\right)$, that is, such that $\nu\left(o_{i}\right) \in \mathcal{M}\left(T_{i}\right)$, one has

$$
\text { either } \quad\left(r_{i}, \nu\right) \in E \text { and } s, \nu \models p_{i} \quad \text { or } \quad\left(r_{i}, \nu\right) \notin E \text { and } s, \nu \models q_{i} \text {. }
$$

We can now state Lemma 8.3, by which the premises of proof rule (PR1) ensure that an execution of a rule program that starts in a configuration consistent with the unary correctness formulas considered, results in a configuration equally unary cf-consistent.

Lemma 8.3. Let $\mathcal{R}=\left\{r_{1}, \ldots, r_{n}\right\}$ be a rule program made of unary rules, and for $i=1, \ldots, n$ let $p_{i}, q_{i} \in \mathcal{F}\left(r_{i}\right)$ be assertions focused on the rules, such that the following correctness formulas hold:

$$
\left.\begin{array}{rl} 
& \left\{p_{i}\right\} r_{i}\left\{q_{i}\right\} \quad \text { for } i=1, \ldots, n \\
\left\{p_{i} \wedge p_{j}\right\} r_{j}\left\{p_{i}\right\} \\
\left\{q_{i} \wedge p_{j}\right\} r_{j}\left\{q_{i}\right\}
\end{array}\right\} \text { for } i, j=1, \ldots, n \text { with } i \neq j .
$$

In an execution $\langle E, s\rangle \stackrel{\mathcal{R}}{\longrightarrow}\left\langle E^{\prime}, s^{\prime}\right\rangle$ of the rule program, if $\langle E, s\rangle$ is consistent with the correctness formulas $\left\{p_{i}\right\} r_{i}\left\{q_{i}\right\}$ then $\left\langle E^{\prime}, s^{\prime}\right\rangle$ is as well.

Proof. The lemma is proven by induction on the length of executions of $\mathcal{R}$.

Base step. If the execution is empty, that is, $\left\langle E^{\prime}, s^{\prime}\right\rangle$ is equal to $\langle E, s\rangle$, then the lemma holds by hypothesis.

Induction step. We consider an execution $\left\langle E_{0}, s_{0}\right\rangle \stackrel{\mathcal{R}}{\longrightarrow}\left\langle E_{k}, s_{k}\right\rangle$ of length $k \geq 1$ of the rule program, such that $\left\langle E_{0}, s_{0}\right\rangle$ is unary cf-consistent, and we assume that $\left\langle E_{k-1}, s_{k-1}\right\rangle$ is unary cf-consistent.

Let $\left\langle E_{k-1}, s_{k-1}\right\rangle \stackrel{R_{k}}{\longrightarrow}\left\langle E_{k}, s_{k}\right\rangle$ be the last transition of this execution, where $R_{k}=\left(r_{i_{k}}, O_{k}\right)$ with $i_{k} \in\{1, \ldots, n\}$ and $O_{k} \in \mathcal{M}\left(T_{i_{k}}\right)$ by the definitions of Section 5.4.

Let us also consider some $i \in\{1, \ldots, n\}$ and some valuation $\nu$, such that $\nu\left(o_{i}\right) \in \mathcal{M}\left(T_{i}\right)$. We examine in turn the cases where the rule instance $R=\left(r_{i}, \nu\right)$ is equal to the rule instance $R_{k}$ used in the last transition, or not; and if not, why. 
- First case: $R=R_{k}$, that is, $R_{k}=\left(r_{i}, \nu\right)$. From $\left\langle E_{k-1}, s_{k-1}\right\rangle \stackrel{R_{k}}{\longrightarrow}\left\langle E_{k}, s_{k}\right\rangle$ and the transition rule (6.1), we have

$$
\begin{gathered}
\left(r_{i}, \nu\right) \in E_{k-1} \\
s_{k-1} \stackrel{\left(r_{i}, \nu\right)}{\longrightarrow} s_{k} \\
\left(r_{i}, \nu\right) \notin E_{k} .
\end{gathered}
$$

From (c) and the hypothesis that $\left\langle E_{k-1}, s_{k-1}\right\rangle$ is unary cf-consistent, we have $s_{k-1}, \nu \models p_{i}$. By (a) and (d), this gives

$$
s_{k}, \nu \models q_{i} .
$$

- Second and third cases: $R \neq R_{k}$, that is, $\left(r_{i}, \nu\right) \neq\left(r_{i_{k}}, O_{k}\right)$. By the transition rule (6.1), a consequence is that if $R \in E_{k-1}$ then $R \in E_{k}$ and if $R \notin E_{k-1}$ then $R \notin E_{k}$.

Let us note $\varphi \equiv p_{i}$ when $R \in E_{k-1}$, and $\varphi \equiv q_{i}$ when $R \notin E_{k-1}$. Since by hypothesis $\left\langle E_{k-1}, s_{k-1}\right\rangle$ is unary cf-consistent, we have $s_{k-1}, \nu \models \varphi$.

We now examine the cases where $i=i_{k}$ and $i \neq i_{k}$.

- Second case: $R \neq R_{k}$ but still $i=i_{k}$. Then we must have $\nu\left(o_{i}\right) \neq O_{k}$.

Consider another valuation $\nu^{\prime}$, such that $\nu^{\prime}\left(o_{i}\right)=O_{k}$ and $\nu^{\prime}(x)=\nu\left(o_{i}\right)$ for some fresh variable $x$ : since $\nu\left(o_{i}\right) \neq O_{k}$ and hence $\nu^{\prime}(x) \neq \nu^{\prime}\left(o_{i}\right)$, we have $\nu^{\prime} \models x \neq o_{i}$. By Lemma 4.2 we have $s_{k-1}, \nu^{\prime} \models \varphi(x)$. Since $s_{k-1} \stackrel{\left(r_{i}, \nu^{\prime}\right)}{\longrightarrow} s_{k}$ and both $r_{i}$ and $\varphi$ are unary, by Lemma 8.1 we have $s_{k}, \nu^{\prime} \models \varphi(x)$. That is by Lemma 4.2 again,

$$
s_{k}, \nu \models \varphi .
$$

- Third case: $R \neq R_{k}$ and $i \neq i_{k}$. Consider a valuation $\nu^{\prime}$ such that $\nu^{\prime}\left(o_{i}\right)=$ $\nu\left(o_{i}\right)$ and $\nu^{\prime}\left(o_{i_{k}}\right)=O_{k}$. Note that we can have $\nu^{\prime}\left(o_{i}\right)=\nu^{\prime}\left(o_{i_{k}}\right)$ or not. However we always have $R_{k}=\left(r_{i_{k}}, \nu^{\prime}\right)$.

By the transition rule $(6.1)$ we have $\left(r_{i_{k}}, \nu^{\prime}\right) \in E_{k-1}$. Since by hypothesis $\left\langle E_{k-1}, s_{k-1}\right\rangle$ is unary cf-consistent, we have

$$
s_{k-1}, \nu^{\prime} \models p_{i_{k}} .
$$

By Lemma 4.1, $s_{k-1}, \nu \models \varphi$ gives

$$
s_{k-1}, \nu^{\prime} \models \varphi .
$$

By (b) we have $\left\{\varphi \wedge p_{i_{k}}\right\} r_{i_{k}}\{\varphi\}$. With (f) and (g) this gives $s_{k}, \nu^{\prime} \models \varphi$. That is by Lemma 4.1 again,

$$
s_{k}, \nu \models \varphi \text {. }
$$

- Summary. We have considered an arbitrary $i \in\{1, \ldots, n\}$ and an arbitrary valuation $\nu$, such that $\nu\left(o_{i}\right) \in \mathcal{M}\left(T_{i}\right)$.

In the first case, we have shown that $s_{k}, \nu \models q_{i}$ while $\left(r_{i}, \nu\right) \notin E_{k}$ by (e).

In the second and third cases, we have shown that $s_{k}, \nu \models \varphi$, where $\varphi \equiv p_{i}$ when $\left(r_{i}, \nu\right) \in E_{k}$ and $\varphi \equiv q_{i}$ when $\left(r_{i}, \nu\right) \notin E_{k}$.

In all cases we have therefore shown that $\left\langle E_{k}, s_{k}\right\rangle$ is unary cf-consistent, which proves the lemma. 
Proof of Theorem 8.2. Let $\mathcal{R}=\left\{r_{1}, \ldots, r_{n}\right\}$ be a rule program made of unary rules, and for $i=1, \ldots, n$ let $p_{i}, q_{i} \in \mathcal{F}\left(r_{i}\right)$ be assertions focused on the rules, such that the following correctness formulas hold:

$$
\left.\begin{array}{rl} 
& \left\{p_{i}\right\} r_{i}\left\{q_{i}\right\} \quad \text { for } i=1, \ldots, n \\
\left\{p_{i} \wedge p_{j}\right\} r_{j}\left\{p_{i}\right\} \\
\left\{q_{i} \wedge p_{j}\right\} r_{j}\left\{q_{i}\right\}
\end{array}\right\} \text { for } i, j=1, \ldots, n \text { with } i \neq j .
$$

Let also $s$ be a $\mathcal{T}$-compliant state in which the precondition of the correctness formula to prove holds, that is,

$$
s \models \bigwedge_{i=1}^{n} \forall o_{i}: T_{i} p_{i} .
$$

Finally let $s^{\prime}$ be a state that results from the execution of $\mathcal{R}$ from $s$, that is, $s \stackrel{\mathcal{R}}{\rightarrow} s^{\prime}$.

Each conjunct in (b) involves a typed quantification, defined in Section 4.1.4. By this definition, for any $i \in\{1, \ldots, n\}$ we have $s, \nu \models p_{i}$ for any valuation $\nu$ such that $\nu\left(o_{i}\right) \in \mathcal{M}\left(T_{i}\right)$.

For all of these $i$ and $\nu$, the rule instance $\left(r_{i}, \nu\right)$ belongs to the set $\mathrm{RI}_{s}(\mathcal{R})$ of all the instances of rules from the rule program $\mathcal{R}$ that are well-typed in $s$, a set introduced in Section 5.4.2.

By definition, $\left\langle\mathrm{RI}_{s}(\mathcal{R}), s\right\rangle$ is the initial configuration of all executions of $\mathcal{R}$ from $s$. The last two statements above express that this configuration is consistent with the correctness formulas $\left\{p_{i}\right\} r_{i}\left\{q_{i}\right\}$ for $i=1, \ldots, n$. Consider the set $E^{\prime}$ of all eligible rule instances at the end of the execution from $s$ to $s^{\prime}$. By Lemma 8.3, the final configuration $\left\langle E^{\prime}, s^{\prime}\right\rangle$ is also consistent with these correctness formulas.

It follows that, for any $i \in\{1, \ldots, n\}$ and any valuation $\nu$ such that $\nu\left(o_{i}\right) \in$ $\mathcal{M}\left(T_{i}\right)$, we have $s^{\prime}, \nu \models q_{i}$. Indeed, consider the rule instance $R=\left(r_{i}, \nu\right)$ :

(i) If $R \in E^{\prime}$ then since $\left\langle E^{\prime}, s^{\prime}\right\rangle$ is unary cf-consistent, we have $s^{\prime}, \nu \models p_{i}$. Since $s^{\prime}$ is the final state of the execution of $\mathcal{R}$, the rule instance $R$ is not applicable in $s^{\prime}$, meaning that $s^{\prime} \stackrel{R}{\rightarrow} s^{\prime}$. Thus by (a) we have $s^{\prime}, \nu \models q_{i}$.

(ii) If $R \notin E^{\prime}$ then since $\left\langle E^{\prime}, s^{\prime}\right\rangle$ is unary cf-consistent, we have $s^{\prime}, \nu \models q_{i}$.

Therefore, by definition of typed quantification again, we have

$$
s^{\prime} \models \bigwedge_{i=1}^{n} \forall o_{i}: T_{i} q_{i}
$$

which proves the conclusion of proof rule (PR1), and ends the proof of the theorem.

As a practical note, it is often the case in programs made of unary rules that the sole variables of the rules in the program all have the same type. The preand postconditions of the correctness formula in the conclusion of the proof rule can use this as follows. If $r_{i}=\left(o_{i}, T, g_{i}, a_{i}\right)$ for $i=1, \ldots, n$, then the proof rule 
can be written

$$
\begin{gathered}
\left\{p_{i}\right\} r_{i}\left\{q_{i}\right\} \quad \text { for } i=1, \ldots, n \\
\left.\left\{p_{i} \wedge p_{j}\right\} r_{j}\left\{p_{i}\right\}\right\} \text { for } i, j=1, \ldots, n \text { with } i \neq j \\
\left\{q_{i} \wedge p_{j}\right\} r_{j}\left\{q_{i}\right\}
\end{gathered}
$$

\subsection{Usage}

This section discusses the approach to adopt when putting the verification method into practice. The successive steps are identified and discussed. The central task resides in finding the proper pre- and postconditions focused on the individual rules.

The method is then illustrated in detail on an example.

Consider a rule program responsible for assigning a bonus to customers based on their ages, according to the function depicted in Fig. 8.1. The rule program $\mathcal{R}=\left\{r_{1}, r_{2}\right\}$ below implements this specification.

$$
\begin{aligned}
& r_{1}(x: P): x \cdot \text { age } \leq 50 \rightarrow x \cdot \text { bonus }:=x \cdot \text { age }+10 \\
& r_{2}(y: P): y \cdot \text { age }>50 \rightarrow y \cdot \text { bonus }:=y \cdot \text { age } \times 2-40
\end{aligned}
$$

This program contains only unary rules. To avoid any confusion, we have given a different name to the variable of each rule.

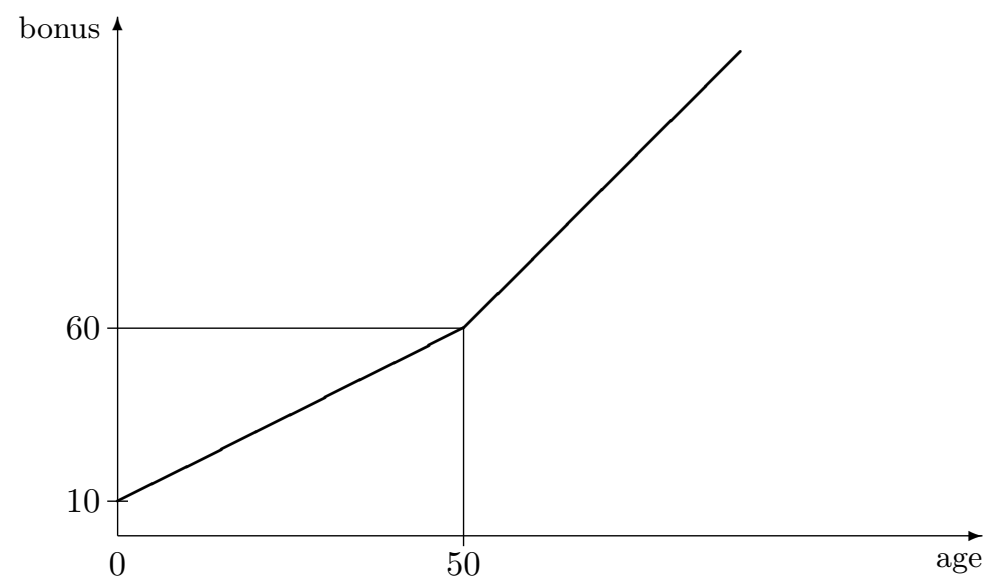

Figure 8.1: Bonus depending on the age.

Imagine we have to certify that, to a set of customers with ages between 20 and 80 , the rule program $\mathcal{R}$ will always assign bonuses between 30 and 120 . This amounts to proving the following correctness formula:

$$
\{\forall o: P(\text { o.age } \in[20,80])\} \mathcal{R}\{\forall o: P(\text { o.bonus } \in[30,120])\} .
$$




\subsubsection{Use the Proof Rule Bottom-Up}

Given a correctness formula for a rule program, the proof task consists in finding two assertions per rule in the program. These assertions must form valid correctness formulas focused on the rules. The resulting correctness formulas must be interference free. In addition, they must lead to the correctness formula to prove, by means of the proof rule on rule programs, and possibly of other proof rules, such as the (CONSEQUENCE) one exposed in Section 6.1.2.

We call this approach bottom-up because it starts from the correctness formula to prove, which appears in the conclusion of the proof rule, and it looks for the correctness formulas appearing in the premises.

In contrast, adopting a top-down approach on the rule program $\mathcal{R}$ presented above would be of little efficiency. Indeed, if we start by expressing correctness formulas for the rules, we will probably propose

$$
\begin{aligned}
& \{x . \text { age } \leq 50\} r_{1}\{x . \text { bonus }=x . \text { age }+10\} \\
& \{y . \text { age }>50\} r_{2}\{y . \text { bonus }=2 \times \text { y.age }-40\}
\end{aligned}
$$

which are not interference free, as each postcondition is invalidated by the other rule. We will then perhaps weaken the postconditions to achieve interference freedom, and propose

$$
\begin{aligned}
& \{x . \text { age } \leq 50\} r_{1}\{x . \text { bonus }=x . \text { age }+10 \vee x . \text { bonus }=2 \times x . \text { age }-40\} \\
& \{y . \text { age }>50\} r_{2}\{y . \text { bonus }=y . \text { age }+10 \vee y . \text { bonus }=2 \times y . \text { age }-40\} .
\end{aligned}
$$

However, since these correctness formulas have incompatible preconditions, one can only derive a correctness formula whose precondition is false from them, which is of little use.

On the other hand, weakening the preconditions would force us to account in the postconditions for the executions of the rules where the guards do not hold. And since in these cases we cannot say anything on the value of the bonus, we seem to be unable to propose interesting correctness formulas that hold on the individual rules.

\subsubsection{What to Look for}

The bottom-up approach means that, given a correctness formula $\{p\} \mathcal{R}\{q\}$ to prove on a rule program $\mathcal{R}=\left\{r_{1}, \ldots, r_{n}\right\}$ made of unary rules, we are going to look for assertions $p_{i}, q_{i}$ that meet the following criteria for $i=1, \ldots, n$ :

(i) The assertions are focused on the corresponding rule, that is, one has $p_{i}, q_{i} \in \mathcal{F}\left(r_{i}\right)$.

(ii) Each correctness formula holds, that is, one has $\left\{p_{i}\right\} r_{i}\left\{q_{i}\right\}$.

(iii) The correctness formulas are interference free, that is, for $j=1, \ldots, n$ with $j \neq i$, one has $\left\{p_{i} \wedge p_{j}\right\} r_{j}\left\{p_{i}\right\}$ and $\left\{q_{i} \wedge p_{j}\right\} r_{j}\left\{q_{i}\right\}$.

(iv) The correctness formula derived by the proof rule leads to the target one, that is, $p \rightarrow\left(\bigwedge_{i=1}^{n} \forall o_{i}: T_{i} p_{i}\right)$ and $\left(\bigwedge_{i=1}^{n} \forall o_{i}: T_{i} q_{i}\right) \rightarrow q$.

In the search for the assertions $p_{i}, q_{i}$, one will draw inspiration from the preand postconditions of the correctness formula to prove and from the effect of the rule actions on the assertions proposed so far. 
From a practical standpoint, each time a correctness formula for a rule is to be verified in steps (ii) and (iii), one must consider both the case where the guard of the rule is satisfied and the case where it is not. Furthermore, in step (iii) the correctness formulas involve other variables that the rule variables. Then the cases where these variables equal, or do not equal, the rule variables must be both considered. However, the following lemma gives a useful shortcut, especially for the correctness formulas that express the interference freedom conditions.

Lemma 8.4. Given a correctness formula $\{p\} r\{q\}$ focused on a unary rule $r=(o, T, g, a)$, and a unary assertion $\varphi \in \mathcal{F}(\{x\})$ in some variable $x$, if

$$
\{\varphi \wedge p \wedge g \wedge x=o\} r\{\varphi\}
$$

then

$$
\{\varphi \wedge p\} r\{\varphi\}
$$

Proof. Consider two states $s$ and $s^{\prime}$ and a valuation $\nu$, such that $s, \nu \models \varphi \wedge p$ and $s \stackrel{(r, \nu)}{\longrightarrow} s^{\prime}$.

- If $s, \nu \models g$ and $\nu \models x=o$ then by hypothesis $s^{\prime}, \nu \models \varphi$.

- If $s, \nu \models g$ and $\nu \models x \neq o$ then by Lemma 8.1 we have $s^{\prime}, \nu \models \varphi$.

- If $s, \nu \models \neg g$ then we have $s^{\prime}=s$ and hence $s^{\prime}, \nu \models \varphi$.

We thus have $\{\varphi \wedge p\} r\{\varphi\}$ in all cases, which proves the lemma.

This lemma is useful when applying the method, to verify such interference freedom conditions as $\left\{p_{i} \wedge p_{j}\right\} r_{j}\left\{p_{i}\right\}$. Here, the assertion $p_{i}$ plays the role of $\varphi$ and the variable $x$ is here $o_{i}$. By the lemma, to verify this correctness formula, it suffices to verify that $\left\{p_{i}\left(o_{j}\right) \wedge p_{j} \wedge g_{j}\right\} r_{j}\left\{p_{i}\left(o_{j}\right)\right\}$ holds.

\subsubsection{Application of the Verification Method}

Let us remind the rule program $\mathcal{R}=\left\{r_{1}, r_{2}\right\}$, which computes bonuses based on ages according to the function depicted in Fig. 8.1.

$$
\begin{aligned}
& r_{1}(x: P): x \cdot a g e \leq 50 \rightarrow x \cdot \text { bonus }:=x \cdot \text { age }+10 \\
& r_{2}(y: P): y \cdot \text { age }>50 \rightarrow y \cdot \text { bonus }:=y \cdot \text { age } \times 2-40
\end{aligned}
$$

We want to prove the following correctness formula for $\mathcal{R}$, expressing that to a set of customers with ages between 20 and 80 , the rule program will always assign bonuses between 30 and 120. In the rest of this example, we note $a$ the age attribute and $b$ the bonus.

$$
\{\forall o: P(o . a \in[20,80])\} \mathcal{R}\{\forall o: P(o . b \in[30,120])\}
$$

Adopting the approach described in this section, we are looking for a preand a postcondition for each rule, such that:

(i) The correctness formulas for $r_{1}$ and $r_{2}$ that can be formed with these pre- and postconditions hold.

(ii) These correctness formulas are interference free. 
(iii) By the proof rule, they lead to the global correctness formula (8.1).

As mentioned in Section 8.4.2, we shall draw inspiration from the pre- and postconditions of the target correctness formula to find the assertions focused on the rules. In our case, we see from the text of the rules, or from Fig. 8.1, that the lower bound 30 on the bonus in the postcondition of (8.1) comes from the application of $r_{1}$ to the lower bound 20 on the age in the precondition. Similarly, a bonus of 120 results from the application of $r_{2}$ to an age of 80 . Hence the idea of dispatching the precondition of (8.1) over the two rules, with $x . a \geq 20$ as the precondition for $r_{1}$ and $y . a \leq 80$ as the one for $r_{2}$. The postconditions are obtained by symbolically executing the rules on their preconditions. In this, one must not forget to take into account both the case where the rule is applicable, and the case where it is not.

We thus propose the following assertions as pre- and postconditions of the correctness formulas focused on each rule:

$$
\begin{array}{ll}
p_{1} \equiv x . a \geq 20 & q_{1} \equiv x . b \in[30,60] \vee x . a>50 \\
p_{2} \equiv y . a \leq 80 & q_{2} \equiv y . b \in(60,120] \vee y . a \leq 50 .
\end{array}
$$

\section{The individual correctness formulas hold}

We start by verifying that the correctness formula $\left\{p_{1}\right\} r_{1}\left\{q_{1}\right\}$ holds. Consider two states $s$ and $s^{\prime}$ and a valuation $\nu$, such that $s, \nu \models p_{1}$ and $s \stackrel{\left(r_{1}, \nu\right)}{\longrightarrow} s^{\prime}$. As described by the semantics of rule instance execution (see Section 5.3.4), the definition of $s^{\prime}$ depends on the applicability of $\left(r_{1}, \nu\right)$ in $s$. That is, on whether $s, \nu \models x . a \leq 50$ or not. Combined with the precondition $p_{1}$, we have

$$
\begin{aligned}
\text { either } & s, \nu \models x . a \in[20,50] & \text { and then } & \left\langle s, s^{\prime}\right\rangle, \nu \models x . b^{\prime}=x . a+10 \\
\text { or } & s, \nu \models x . a>50 & \text { and then } \quad & s=s^{\prime} .
\end{aligned}
$$

The first case gives $s^{\prime}, \nu \models x . b \in[30,60]$, so in all cases we have $s^{\prime}, \nu \models q_{1}$. As a consequence $\left\{p_{1}\right\} r_{1}\left\{q_{1}\right\}$ holds.

A similar reasoning gives that $\left\{p_{2}\right\} r_{2}\left\{q_{2}\right\}$ holds.

\section{Interference freedom}

We start by verifying that $\left\{p_{1} \wedge p_{2}\right\} r_{2}\left\{p_{1}\right\}$ holds. By Lemma 8.4 it suffices to verify that $\left\{p_{1}(y) \wedge p_{2} \wedge g_{2}\right\} r_{2}\left\{p_{1}(y)\right\}$ holds, that is,

$$
\{y . a \geq 20 \wedge y . a \leq 80 \wedge y . a>50\} r_{2}\{y . a \geq 20\} .
$$

This correctness formula is simply $\{y . a \in(50,80]\} r_{2}\{y . a \geq 20\}$, which holds obviously since $r_{2}$ does not update the age. A similar reasoning gives that $\left\{p_{2} \wedge p_{1}\right\} r_{1}\left\{p_{2}\right\}$ holds.

To verify $\left\{q_{1} \wedge p_{2}\right\} r_{2}\left\{q_{1}\right\}$ we use Lemma 8.4 again, and we only verify that $\left\{q_{1}(y) \wedge p_{2} \wedge g_{2}\right\} r_{2}\left\{q_{1}(y)\right\}$ holds, that is,

$$
\{(y . b \in[30,60] \vee y . a>50) \wedge y . a \in(50,80]\} r_{2}\{y . b \in[30,60] \vee y . a>50\} .
$$

Let us consider an execution $s \stackrel{\left(r_{2}, \nu\right)}{\longrightarrow} s^{\prime}$ of an instance of $r_{2}$ from a state that satisfies the precondition of this correctness formula. In particular, we have $s, \nu \models y . a>50$. Since $r_{2}$ does not update the age, we also have $s^{\prime}, \nu \models y . a>50$, which implies $s^{\prime}, \nu \models q_{1}(y)$. As a consequence $\left\{q_{1} \wedge p_{2}\right\} r_{2}\left\{q_{1}\right\}$ holds. A similar reasoning gives $\left\{q_{2} \wedge p_{1}\right\} r_{1}\left\{q_{2}\right\}$. 


\section{Proving the targeted correctness formula}

Having verified all the premises of proof rule (PR1), we can derive its conclusion, that is,

$$
\begin{aligned}
&\{\forall o: P(o . a \geq 20\wedge o . a \leq 80)\} \mathcal{R} \\
&\{\forall o: P((o . b \in[30,60] \vee o . a>50) \wedge(o . b \in(60,120] \vee o . a \leq 50))\} .
\end{aligned}
$$

The precondition of this correctness formula is directly equivalent to $\forall o: P$ (o.a $\in$ $[20,80])$ and the postcondition implies $\forall o: P(o . b \in[30,120])$.

We have thus proven the targeted correctness formula for $\mathcal{R}$, and by doing so we have certified that to a set of customers with ages between 20 and 80 , the rule program will always assign bonuses between 30 and 120 .

\subsection{Incompleteness}

The simplifying setting used in this chapter to present our verification method includes using unary rules, and also not taking eligibility into account. The latter causes the proof rule introduced in Section 8.3 to not be complete. In contrast, the proof rule proposed in the next chapter for the general case is relatively complete (in the sense defined there).

Proof rule (PR1) is sound, meaning that all the correctness formulas that are derived by this proof rule are valid. However, it is not complete, meaning that there are valid correctness formulas for some rule programs made of unary rules, for which no proof can be found using the proof rule. That is, for such a correctness formula one cannot find individual correctness formulas focused on the rules that hold, are interference free, and whose combination leads to the correctness formula for the rule program. This property is formalized by Theorem 8.5.

Theorem 8.5. The proof rule (PR1) is not complete.

Proof. To prove the theorem, we exhibit a counterexample.

Consider the rule program $\mathcal{R}=\left\{r_{1}, r_{2}\right\}$ made of the following rules, noting $r_{1}=\left(x, \mathrm{~T}, g_{1}, a_{1}\right)$ and $r_{2}=\left(y, \mathrm{~T}, g_{2}, a_{2}\right)$ :

$$
\begin{aligned}
& r_{1}(x: T): x \cdot a \geq 20 \rightarrow x \cdot b:=x \cdot a-10 \\
& r_{2}(y: T): y \cdot b>70 \rightarrow y \cdot b:=70
\end{aligned}
$$

The following correctness formula for $\mathcal{R}$ states that, when executed from a state where all bonuses are zero, the rule program leads to a state where no bonus is greater than 70. It is formally proven in Section 10.1.1, but its validity is quite obvious to the trained eye.

$$
\{\forall o: \mathrm{T}(o . b=0)\} \mathcal{R}\{\forall o: \mathrm{T}(o . b \leq 70)\} .
$$

However, this correctness formula cannot be proven using proof rule (PR1), which we show below by contradiction.

Indeed, suppose that this correctness formula can be proven using proof rule (PR1). Then there exist assertions $p_{1}, q_{1} \in \mathcal{F}\left(r_{1}\right)$ and $p_{2}, q_{2} \in \mathcal{F}\left(r_{2}\right)$ that form 
valid, interference free correctness formulas for the rules $r_{1}$ and $r_{2}$, and such that in addition

$$
\begin{aligned}
\forall o: \mathrm{T}(o . b=0) & \rightarrow \forall o: \mathrm{T}\left(p_{1}(o) \wedge p_{2}(o)\right) \\
\forall o: \mathrm{T}\left(q_{1}(o) \wedge q_{2}(o)\right) & \rightarrow \forall o: \mathrm{T}(o . b \leq 70) .
\end{aligned}
$$

Consider the following working memory, states and valuation:

$$
\begin{array}{rlrl}
\mathcal{M}(\mathrm{T})=\{\mathrm{X}\} & & \\
s_{1}: & a^{s_{1}}(\mathrm{X})=81 & b^{s_{1}}(\mathrm{X})=0 \\
s_{2}: & a^{s_{2}}(\mathrm{X})=81 & b^{s_{2}}(\mathrm{X})=71 \\
\nu: & x \mapsto \mathrm{X} \quad y \mapsto \mathrm{X} \quad o \mapsto \mathrm{X} .
\end{array}
$$

In the state $s_{1}$ the value of the attribute $b$ on the only instance $\mathrm{X}$ of the type $\mathrm{T}$ is 0 , so we have $s_{1} \models \forall o: T(o . b=0)$. By (b) this gives $s_{1} \models \forall o: T\left(p_{1}(o) \wedge p_{2}(o)\right)$, hence

$$
\begin{aligned}
& s_{1}, \nu \models p_{1} \\
& s_{1}, \nu \models p_{2} .
\end{aligned}
$$

In addition, the states $s_{1}$ and $s_{2}$ have been defined so that $s_{1}, \nu \models g_{1}$ and $\left\langle s_{1}, s_{2}\right\rangle, \nu \models \hat{a}_{1}$. Therefore, we have

$$
s_{1} \stackrel{\left(r_{1}, \nu\right)}{\longrightarrow} s_{2}
$$

Since the correctness formula $\left\{p_{1}\right\} r_{1}\left\{q_{1}\right\}$ holds by hypothesis, from (d) and (f) we have

$$
s_{2}, \nu \models q_{1} .
$$

On the other hand, the state $s_{1}$ is such that $s_{1}, \nu \not \models g_{2}$. A consequence is that $s_{1} \stackrel{\left(r_{2}, \nu\right)}{\longrightarrow} s_{1}$. Since the correctness formula $\left\{p_{2}\right\} r_{2}\left\{q_{2}\right\}$ holds by hypothesis, this with (e) give that

$$
s_{1}, \nu \models q_{2} .
$$

Another part of the hypothesis is that the interference freedom correctness formula $\left\{q_{2} \wedge p_{1}\right\} r_{1}\left\{q_{2}\right\}$ holds. Therefore, from (h), (d) and (f), we have $s_{2}, \nu \models$ $q_{2}$. This and (g) give by Lemma 4.2 that $s_{2}, \nu \models q_{1}(o) \wedge q_{2}(o)$. By Lemma 4.1, and due to the fact that $\mathcal{M}(\mathrm{T})$ is a singleton, this means that $s_{2}, \nu^{\prime} \models q_{1}(o) \wedge$ $q_{2}(o)$ for any valuation $\nu^{\prime}$ such that $\nu^{\prime}(o) \in \mathcal{M}(\mathrm{T})$, which is the definition of

$$
s_{2} \models \forall o: \mathrm{T}\left(q_{1}(o) \wedge q_{2}(o)\right) .
$$

By (c) this gives $s_{2} \models \forall o$ : $\mathrm{T}(o . b \leq 70)$, which contradicts $b^{s_{2}}(\mathrm{X})=71$.

We have thus exhibited a valid correctness formula that cannot be proven using proof rule (PR1). We have shown this by demonstrating that otherwise, a false statement (namely $71 \leq 70$ ) would follow.

In this proof, the contradiction is built by considering an execution of $\mathcal{R}$ in which an instance of $r_{1}$ is applied after an instance of $r_{2}$ to the same object X. The interference freedom between $r_{1}$ and the postcondition of the correctness formula focused on $r_{2}$ is expressed by the premise $\left\{q_{2} \wedge p_{1}\right\} r_{1}\left\{q_{2}\right\}$ of the proof rule. It is exploited after step (h) to lead to the contradiction. 
However, this execution of the rule program is not possible, because the applications of $\left(r_{2}, \mathrm{X}\right)$, and then of $\left(r_{1}, \mathrm{X}\right)$, are considered on an object $\mathrm{X}$ with the initial attribute value $b^{s_{1}}(\mathrm{X})=0$. In this initial state, only $r_{1}$ is applicable to $\mathrm{X}$. And once the rule instance $\left(r_{1}, \mathrm{X}\right)$ has been applied, it becomes ineligible, and hence will never be applied again. Therefore, there can be no execution of $\mathcal{R}$ from a state $s_{1}$ where $b^{s_{1}}(\mathrm{X})=0$, in which the rule instance $\left(r_{1}, \mathrm{X}\right)$ is applied after $\left(r_{2}, \mathrm{X}\right)$.

The incompleteness of the proof rule manifests itself in the fact that the premises of the proof rule do not discard such an impossible execution.

Eligibility plays a central role here. The premises of the proof rule do not take it into account, which causes the proof rule to fail proving a correctness formula when eligibility has to be relied upon. Proof rule (PR2) introduced in Chapter 9 does take eligibility into account, and is complete - actually relatively complete, in the sense discussed in Section 9.4. Its specialization (PR3) for unary rules (given in Section 10.1.1) is also relatively complete for rule programs made of unary rules. 


\section{Chapter 9}

\section{A Verification Method for Rule Programs}

In the previous chapter, we have introduced a proof rule suited to rule programs made of unary rules. We used this proof rule to expose the core principle of our verification method: derive a correctness formula for a rule program from interference-free correctness formulas focused on the individual rules in the program.

However, this first proof rule has two limitations. Firstly, it applies only to rule programs made of unary rules. Secondly, it does not take the eligibility of rule instances into account, hence it is not complete.

The present chapter proposes a proof rule that does not suffer from these limitations. On the one hand, relieving the constraint for rules to be unary brings back the possibility of interference due to aliasing. This possibility is addressed through additional premises in the proof rule. On the other hand, eligibility of rule instances is taken into account by the introduction of dedicated ghost variables. As a result, we obtain a verification method that handles rules of arbitrary arity, and that is relatively complete (in the sense discussed in Section 9.4)

As since the start of Part II, we work within the setting exposed by Section 6.1, that is, rule programs are executed under the one-shot execution strategy and we use the classical definitions of proofs and proof rules, as reminded in that section.

\subsection{Taking Eligibility into Account}

In order to propose a verification method suited for the general case of rule programs, eligibility must be taken into account. To this end, we extend our formal framework with ghost variables that embody the eligibility of rule instances. The present section defines these eligibility ghost variables, as well as eligibility-aware version of assertions, execution, and correctness formulas. 


\subsubsection{Eligibility Ghost Variables}

With each rule in a rule program, we associate a ghost variable with a precise interpretation that binds instances of this ghost variable to the eligibility of instances of the rule. These eligibility ghost variables follow the definitions of Section 7.2.1, which introduced ghost variables. We remind the main points below.

(i) Ghost variables are introduced by augmenting the signature $\Sigma$ (Section 3.1) with an infinite set of function symbols, noted Ghost.

(ii) They are incorporated into expressions and assertions by defining extended versions of the corresponding languages: Fig. 7.2 defines the languages $\mathcal{E}(V, G)$ and $\mathcal{A}(V, G)$ of expressions and assignment; the definition of the languages $\mathcal{B}(V, G)$ and $\mathcal{F}(V, G)$ of non-quantified and quantified formulas is based on them. In the above, $V \subseteq$ Var is a set of variables and $G \subseteq$ Ghost is a set of ghost variables.

(iii) A ghost variable is a function symbol. If its arity is $m$, it is interpreted in a state by a function defined on $\mathbb{D}^{m}$ with values in $\mathbb{V} \cup\{\perp\}$.

More specifically, we now define the eligibility ghost variables associated with a rule program.

Definition 9.1. Given a rule program $\mathcal{R}=\left\{r_{1}, \ldots, r_{n}\right\}$, the set of eligibility ghost variables for $\mathcal{R}$ is $\Delta=\left\{\delta_{1}, \ldots, \delta_{n}\right\}$ with $\delta_{i} \in$ Ghost and $\left|\delta_{i}\right|=\left|r_{i}\right|$, for $i=1, \ldots, n$; that is, each $\delta_{i}$ is a ghost variable of the same arity of the rule $r_{i}$. In a configuration $\langle E, s\rangle$, each variable $\delta_{i}$ is interpreted by the function $\delta_{i}^{s}$ defined below, for $i=1, \ldots, n$ :

$$
\begin{aligned}
\delta_{i}^{s}: \mathbb{D}^{\left|r_{i}\right|} & \rightarrow \mathbb{B} \\
\vec{O} & \mapsto \begin{cases}\text { false } & \text { if }\left(r_{i}, \vec{O}\right) \in E \\
\text { true } & \text { if }\left(r_{i}, \vec{O}\right) \notin E .\end{cases}
\end{aligned}
$$

The name $\delta_{i}$ of eligibility ghost variables has been chosen to stand for "done", as it encodes the ineligibility of the rule instance, which in the one-shot strategy occurs when the rule instance has been applied once.

For a given $i \in\{1, \ldots, n\}$, the eligibility ghost variable $\delta_{i}$ names a function whose formal parameters are the variables of the rule $r_{i}$. However, as for ghost variables in Section 7.2.1, its argument list is left implicit when unambiguous, and only the $\delta_{i}$ symbol is used instead of the full $\delta_{i}\left(o_{1}^{i}, \ldots, o_{m_{i}}^{i}\right)$, or $\delta_{i}\left(\vec{o}_{i}\right)$ for short.

A common case where the argument list of the eligibility ghost variable $\delta_{i}$ must be explicited is to refer to the eligibility of an instance of the rule $r_{i}$ on objects held by other variables than those of $r_{i}$. We then note $\delta_{i}(\vec{x})$ the eligibility of an instance of the rule $r_{i}$ on objects held in the $m_{i}$-tuple of variables $\vec{x}$.

Example. Consider the rule program $\mathcal{R}=\left\{r_{1}, r_{2}, r_{3}, r_{4}\right\}$ made of the rules below:

$$
\begin{gathered}
r_{1}\left(p_{1}: P\right): p_{1} \text {.age }>60 \rightarrow p_{1} \text {.bonus }:=p_{1} \text {. bonus }+10 \\
r_{2}\left(p_{2}: P\right): p_{2} \cdot \text { cat }=\text { Gold } \rightarrow p_{2} \text {.bonus }:=p_{2} \cdot \text { bonus }+15 \\
r_{3}\left(c_{3}: C, p_{3}: P\right): p_{3} \text {.bonus }<20 \rightarrow c_{3} \text {.points }:=c_{3} \cdot \text { points }+5 \\
r_{4}\left(c_{4}: C, p_{4}: P\right): p_{4} \text {.bonus } \geq 20 \rightarrow c_{4} \text {.points }:=c_{4} \text {.points }+10
\end{gathered}
$$


The set of eligibility ghost variables for $\mathcal{R}$ is $\Delta=\left\{\delta_{1}, \delta_{2}, \delta_{3}, \delta_{4}\right\}$, where $\delta_{1}$ and $\delta_{2}$ are unary, $\delta_{3}$ and $\delta_{4}$ are binary.

Here, $\delta_{1}$ is a shorthand for $\delta_{1}\left(p_{1}\right)$ and $\delta_{3}$ is a shorthand for $\delta_{3}\left(c_{3}, p_{3}\right)$. The notation $\delta_{4}(c, p)$ would refer to the eligibility of an instance of $r_{4}$ on objects held in the variables $c$ and $p$.

The introduction of eligibility ghost variables reifies the notion of eligibility. It establishes a connection between the set of eligible rule instances and the state in configurations of the abstract machine used to model the execution of a rule program.

In particular, the interpretation of the eligibility ghost variables in Definition 9.1 means that during a transition in an execution of the program, not only the rule instance is removed from the set of eligible rule instances, but the value of the ghost variable associated with the rule also changes from false to true on the objects in the instance:

$$
\text { if }\langle E, s\rangle \stackrel{\left(r_{i}, \vec{O}\right)}{\longrightarrow}\left\langle E^{\prime}, s^{\prime}\right\rangle \quad \text { then } \quad \delta_{i}^{s}(\vec{O})=\text { false } \quad \text { and } \quad \delta_{i}^{s^{\prime}}(\vec{O})=\text { true. }
$$

More generally, eligibility-aware assertions, rule execution, and correctness formulas described in the rest of this section make it possible to model rule program execution with formulas that include eligibility ghost variables. This opens the way to a proof rule that takes eligibility into account, as proposed in Section 9.3.

\subsubsection{Eligibility-Aware Assertions}

The eligibility ghost variables are not meant to be used in the guards or actions of rules, but in the assertions that express properties of rules or of rule programs, in particular as pre- and postconditions of correctness formulas.

These assertions belong to a language of the $\mathcal{F}(V, G)$ family, as introduced in Section 7.2.1. More precisely, given a rule program $\mathcal{R}=\left\{r_{1}, \ldots, r_{n}\right\}$ and the set $\Delta$ of its eligibility ghost variables, the following three languages of so-called eligibility-aware assertions are used, for the following purposes.

- Correctness formulas for an individual rule $r_{i}$ are formed with assertions in the variables of the rule $r_{i}$, possibly involving eligibility ghost variables. This corresponds to the language $\mathcal{F}\left(\operatorname{var}\left(r_{i}\right), \Delta\right)$.

When these assertions are in addition well-typed for $\left(\vec{o}_{i}: \vec{T}_{i}\right)$, we call them eligibility-aware assertions focused on $r_{i}$. We note their set $\mathcal{F}_{\Delta}\left(r_{i}\right)$.

- Correctness formulas expressing the interference freedom between rules are formed with assertions in the variables of several rules of the program, possibly involving eligibility ghost variables. This corresponds to the language $\mathcal{F}(\operatorname{var}(\mathcal{R}), \Delta)$.

- Correctness formulas expressing global properties of the working memory in an execution of a rule program are formed with closed assertions, possibly involving eligibility ghost variables. This corresponds to the language $\mathcal{F}(\emptyset, \Delta)$. 
Example. Given the rules in the previous example, the assertion below expresses that the bonus of the person held in variable $p_{2}$ is 0 if the instance of $r_{1}$ on this person has not yet been applied, and is 10 if the instance of $r_{1}$ on the person held in $p_{2}$ has already been applied.

$$
p_{2} \text {.bonus }=0 \wedge \neg \delta_{1}\left(p_{2}\right) \vee p_{2} . \text { bonus }=10 \wedge \delta_{1}\left(p_{2}\right)
$$

Although it contains occurrences of the eligibility ghost variable for $r_{1}$, its only free (regular) variable is $p_{2}$. It is thus an eligibility-aware assertion focused on rule $r_{2}$, that is, it belongs to $\mathcal{F}_{\Delta}\left(r_{2}\right)$.

An eligibility-aware assertion is given a truth value by a state and a valuation. In addition, in order for the state to interpret eligibility ghost variables as per Definition 9.1, it must be related to a configuration. It is the case in the initial state of a rule program execution. The next section on eligibility-aware execution describes the effect on eligibility ghost variables of a transition between configurations. This description inductively enables all states in a rule program execution to interpret eligibility ghost variables.

\subsubsection{Eligibility-Aware Execution}

The application of rule instances during the execution of a rule program depends, and has an effect, on the eligibility of rule instances. Chapter 5 formalizes this with configurations of an abstract machine. We introduce in this section "eligibility-aware" concepts to encode it with eligibility ghost variables.

\section{Rule instance execution}

Definition 9.2. Let $\mathcal{R}=\left\{r_{1}, \ldots, r_{n}\right\}$ be a rule program and $\Delta$ the set of its eligibility ghost variables; let $r_{i}=\left(\vec{o}_{i}, \vec{T}_{i}, g_{i}, a_{i}\right)$ be a rule in this program and $\nu$ a valuation well-typed for $\left(\vec{o}_{i}: \vec{T}_{i}\right)$.

(i) The eligibility-aware rule instance of $r_{i}$ on the tuple of objects $\vec{O}=$ $\left(\nu\left(o_{1}\right), \ldots, \nu\left(o_{\left|r_{i}\right|}\right)\right)$ is noted $\left(r_{i}^{*}, \nu\left(o_{1}\right), \ldots, \nu\left(o_{\left|r_{i}\right|}\right)\right)$, or $\left(r_{i}^{*}, \vec{O}\right)$ or $\left(r_{i}^{*}, \nu\right)$.

(ii) Given a state $s$, the eligibility-aware rule instance $\left(r_{i}^{*}, \nu\right)$ is selectable in $s$ if the rule instance $\left(r_{i}, \nu\right)$ is applicable in $s$ and $\delta_{i}^{s}(\vec{O})=$ false. That is,

$$
s, \nu \models g_{i} \wedge \neg \delta_{i} .
$$

During the execution of a rule program, the set of selectable rule instances in a configuration $\langle E, s\rangle$ coincides with the rule instances in $E$ that are applicable in $s$. The definition above extends this notion to states that are not considered in the context of a configuration.

The effect of applying a rule instance from a given state is described in Section 5.3.3 as producing a new state, related to the initial one by the transition assertion $\hat{a}$ for the action $a$ of the rule, defined in Section 4.5. We express the effect on the eligibility ghost variables by a similar transition assertion, as follows.

Definition 9.3. Let $\mathcal{R}=\left\{r_{1}, \ldots, r_{n}\right\}$ be a rule program and $\Delta$ the set of its eligibility ghost variables; let $r_{i}=\left(\vec{o}_{i}, \vec{T}_{i}, g_{i}, a_{i}\right)$ be a rule in this program and $\nu$ a valuation well-typed for $\left(\vec{o}_{i}: \vec{T}_{i}\right)$. 
(i) Given a state $s$ in which $\left(r_{i}^{*}, \nu\right)$ is selectable, the state that results from the eligibility-aware application of $\left(r_{i}^{*}, \nu\right)$ in $s$ is the state $s^{\prime}$ such that

$$
\left\langle s, s^{\prime}\right\rangle, \nu \models \hat{a}_{i} \wedge \hat{\delta}_{i}
$$

with the definition given in Section 4.5 of the transition assertion $\hat{a}_{i}$ for the action of $r_{i}$, and with the following definition for the $\hat{\delta}_{i}$ transition assertion:

$$
\hat{\delta}_{i} \stackrel{\text { def }}{\equiv} \delta_{i}^{\prime}\left(\vec{o}_{i}\right) \wedge \forall \vec{o}\left(\vec{o} \neq \vec{o}_{i} \rightarrow \delta_{i}^{\prime}(\vec{o})=\delta_{i}(\vec{o})\right) \wedge \bigwedge_{\delta \in \Delta \backslash\left\{\delta_{i}\right\}} \forall \vec{o}\left(\delta^{\prime}(\vec{o})=\delta(\vec{o})\right) .
$$

(ii) Given a state $s$, the state that results from the eligibility-aware execution of $\left(r_{i}^{*}, \nu\right)$ in $s$ is the state that results from its eligibility-aware application if $\left(r_{i}^{*}, \nu\right)$ is selectable in $s$, and is $s$ itself otherwise. We note $s \stackrel{\left(r_{i}^{*}, \nu\right)}{\longrightarrow} s^{\prime}$ if $s^{\prime}$ is the resulting state.

The definition of the transition assertion $\hat{\delta}_{i}$ for the eligibility ghost variables above formalizes the transition of $\delta_{i}$ from false in state $s$ to true in state $s^{\prime}$, additionally taking into account the chances of aliasing.

Example. Let us focus on the rules $r_{1}$ and $r_{3}$ of the same program as in previous examples:

$$
\begin{gathered}
\mathrm{r}_{1}\left(\mathrm{p}_{1}: \mathrm{P}\right): \mathrm{p}_{1} \text {.age }>60 \rightarrow \mathrm{p}_{1} \text {.bonus }:=\mathrm{p}_{1} \text {. bonus }+10 \\
\mathrm{r}_{3}\left(\mathrm{c}_{3}: \mathrm{C}, \mathrm{p}_{3}: \mathrm{P}\right): \mathrm{p}_{3} \text {.bonus }<20 \rightarrow \mathrm{c}_{3} \text {.points }:=\mathrm{c}_{3} \text {.points }+5
\end{gathered}
$$

Consider the following setting:

$$
\begin{aligned}
& \mathcal{M}(\mathrm{P})=\{\text { Alice }, \mathrm{Bob}\} \\
& \mathcal{M}(\mathrm{C})=\{\text { Card }\} \\
& s_{1}=\text { age }^{s_{1}}: \text { Alice } \mapsto 75 \quad \text { bonus }^{s_{1}}: \text { Alice } \mapsto 15 \\
& \text { Bob } \mapsto 50 \quad \mathrm{Bob} \mapsto 30 \\
& \text { points }^{s_{1}} \text { : Card } \mapsto 25 \\
& \delta_{1}^{s_{1}}: \text { Alice } \mapsto \text { true } \quad \delta_{3}^{s_{1}}: \text { Card, Alice } \mapsto \text { false } \\
& \text { Bob } \mapsto \text { false } \quad \text { Card, Bob } \mapsto \text { true. }
\end{aligned}
$$

In the state $s_{1}$, the eligibility-aware rule instance $\left(r_{1}^{*}\right.$, Alice) is applicable but it is not selectable since $s_{1}, \nu \not \models \neg \delta_{1}$ for any valuation $\nu$ that maps $p_{1}$ onto Alice. In contrast, the eligibility-aware rule instance ( $r_{3}^{*}$, Card, Alice) is selectable.

Let us name $s_{2}$ the state that results from the eligibility-aware application of $\left(r_{3}^{*}\right.$, Card, Alice $)$ from $s_{1}$, that is, $s_{1} \stackrel{\left(r_{3}^{*} \text {,Card,Alice }\right)}{\longrightarrow} s_{2}$. Let us note $\nu$ a valuation that maps $c_{3}$ onto Card and $p_{3}$ onto Alice. Since the rule instance $\left(r_{3}^{*}, \nu\right)$ is selectable, we have $\left\langle s_{1}, s_{2}\right\rangle, \nu \models \hat{a}_{3} \wedge \hat{\delta}_{3}$, that is,

$$
\begin{aligned}
\left\langle s_{1}, s_{2}\right\rangle, \nu \models & c_{3} . \text { points }{ }^{\prime}=c_{3} . \text { points }+5 \wedge \delta_{3}^{\prime}\left(c_{3}, p_{3}\right)=\text { true } \\
& \wedge \forall o\left(o \neq c_{3} \rightarrow c_{3} . \text { points } c^{\prime}=c_{3} . \text { points }\right) \wedge \bigwedge_{f \in \operatorname{Attr} \backslash\{\text { points }\}} \forall o\left(o . f^{\prime}=o . f\right) \\
& \wedge \forall \vec{o}\left(\vec{o} \neq\left(c_{3}, p_{3}\right) \rightarrow \delta_{3}^{\prime}(\vec{o})=\delta_{3}(\vec{o})\right) \wedge \bigwedge_{\delta \in \Delta \backslash\left\{\delta_{3}\right\}} \forall \vec{o}\left(\delta^{\prime}(\vec{o})=\delta(\vec{o})\right) .
\end{aligned}
$$


Which gives the following for $s_{2}$ :

$$
\begin{aligned}
& \begin{aligned}
s_{2}=\text { age }^{s_{2}}: \text { Alice } & \mapsto 75 & \text { bonus }^{s_{2}}: \text { Alice } & \mapsto 15 \\
\text { Bob } & \mapsto 50 & \text { Bob } & \mapsto 30
\end{aligned} \\
& \text { points }^{s_{2}}: \text { Card } \mapsto \mathbf{3 0} \\
& \delta_{1}^{s_{2}} \text { : Alice } \mapsto \text { true } \quad \delta_{3}^{s_{2}} \text { : Card, Alice } \mapsto \text { true } \\
& \text { Bob } \mapsto \text { false } \quad \text { Card, Bob } \mapsto \text { true. }
\end{aligned}
$$

\section{Transition assertions in detail}

Given two states $s$ and $s^{\prime}$, the definition of the eligibility-aware execution of a rule instance $s \stackrel{\left(r_{i}^{*}, \nu\right)}{\longrightarrow} s^{\prime}$ can be summed up as:

$$
\begin{aligned}
& \text { either } \quad s, \nu \models g_{i} \wedge \neg \delta_{i} \quad \text { and then } \quad\left\langle s, s^{\prime}\right\rangle, \nu \models \hat{a}_{i} \wedge \hat{\delta}_{i} \\
& \text { or } \quad s, \nu \models \neg g_{i} \vee \delta_{i} \quad \text { and then } \quad s=s^{\prime}
\end{aligned}
$$

In the above, the equation $s, \nu \models g_{i} \wedge \neg \delta_{i}$ expresses that the rule instance $\left(r_{i}^{*}, \nu\right)$ is selectable. In that case, the resulting state is defined by the transition assertions $\hat{a}_{i}$ and $\hat{\delta}_{i}$. These were introduced by Definitions 4.8 and 9.3 , respectively. The transition assertion $\hat{a}_{i}$ describes the effect of the action of the rule on the attributes, the transition assertion $\hat{\delta}_{i}$ describes the update of the eligibility ghost variables. When $\left(r_{i}^{*}, \nu\right)$ is not selectable, the state that results from the rule execution is defined as identical to the initial one.

In the same way as noted in Section 5.3.3 about the transition assertion for a rule action, the combined transition assertion $\hat{a}_{i} \wedge \hat{\delta}_{i}$ describes the transition for several objects, or sets of objects:

(i) The objects in the rule instance $\left(r_{i}, \nu\right)$ are held by the variables $\vec{o}_{i}$. On the object held by the first variable $o_{1}^{i}$, the attribute $f_{r_{i}}$ is updated to the value $e_{r_{i}}$. Also, the instance of the rule on the tuple of objects becomes ineligible. That is, we have the following in the state $s^{\prime}$ that results from the rule instance application:

$$
\begin{aligned}
& \left\langle s, s^{\prime}\right\rangle, \nu \models o_{1}^{i} \cdot f_{r_{i}}^{\prime}=e_{r_{i}} \\
& \left\langle s, s^{\prime}\right\rangle, \nu \models \delta_{i}^{\prime} \quad \text { that is } \quad s^{\prime}, \nu \models \delta_{i}\left(\vec{o}_{i}\right) .
\end{aligned}
$$

(ii) On any other object than the one held by the first variable $o_{1}^{i}$ of the rule, the value of the attribute $f_{r_{i}}$ is left unchanged. Also, the instances of $r_{i}$ on any other tuple of objects than the ones held by the variables $\overrightarrow{o_{i}}$ keeps its (positive or negative) eligibility. That is, in $s^{\prime}$ we have

$$
\begin{aligned}
& \left\langle s, s^{\prime}\right\rangle, \nu \models o \neq o_{1}^{i} \rightarrow o . f_{r_{i}}^{\prime}=o . f_{r_{i}} \\
& \left\langle s, s^{\prime}\right\rangle, \nu \models \vec{o} \neq \vec{o}_{i} \rightarrow \delta_{i}^{\prime}(\vec{o})=\delta_{i}(\vec{o}) .
\end{aligned}
$$

(iii) For all objects, the value of any other attribute than $f_{r_{i}}$ is left unchanged. The eligibility of all instances of any other rule than $r_{i}$ does not change either. That is, in $s^{\prime}$ we have

$$
\begin{array}{ll}
\left\langle s, s^{\prime}\right\rangle \models \forall o\left(o . f^{\prime}=o . f\right) & \text { for any } f \in \operatorname{Attr} \backslash\left\{f_{r_{i}}\right\} \\
\left\langle s, s^{\prime}\right\rangle \models \forall \vec{o}\left(\delta^{\prime}(\vec{o})=\delta(\vec{o})\right) & \text { for any } \delta \in \Delta \backslash\left\{\delta_{i}\right\} .
\end{array}
$$

These three viewpoints on the effects of a rule instance application will be successively used in many of the proofs of this chapter and of the next. 


\section{Rule and rule program execution}

From the definition of the eligibility-aware execution of a rule instance (Definition 9.3), the eligibility-aware execution of a rule follows naturally.

Definition 9.4. Let $\mathcal{R}=\left\{r_{1}, \ldots, r_{n}\right\}$ be a rule program and $\Delta$ the set of its eligibility ghost variables; let $r_{i}=\left(\vec{o}_{i}, \vec{T}_{i}, g_{i}, a_{i}\right)$ be a rule in this program. Given two states $s$ and $s^{\prime}$, there is an eligibility-aware execution of the rule $r_{i}$ from $s$ to $s^{\prime}$, and we note $s \stackrel{r_{i}^{*}}{\longrightarrow} s^{\prime}$, if there exists an eligibility-aware instance $R^{*}$ of $r_{i}$ such that $s \stackrel{R^{*}}{\longrightarrow} s^{\prime}$.

The execution of rule programs is inherently eligibility-aware: the definition of rule program execution given in Section 5.4 already handles eligibility. However, with the introduction of eligibility ghost variables, the transition rule (5.1), which we specialized for the one-shot strategy as transition rule (6.1), can be expressed as follows. The transition rule (9.1) below describes a transition of the abstract machine from a state $s$ to a state $s^{\prime}$ by the application of an instance of the rule $r_{i}=\left(\vec{o}_{i}, \vec{T}_{i}, g_{i}, a_{i}\right)$ :

$$
\frac{\left\langle s, s^{\prime}\right\rangle, \nu \models \neg \delta_{i} \wedge g_{i} \wedge \hat{a}_{i} \wedge \hat{\delta}_{i}}{\langle E, s\rangle \stackrel{\left(r_{i}, \nu\right)}{\longrightarrow}\left\langle E^{\prime}, s^{\prime}\right\rangle} .
$$

\subsubsection{Eligibility-Aware Correctness Formulas}

Correctness formulas that take into account the eligibility of rule instances, namely eligibility-aware correctness formulas, build upon the eligibility-aware concepts introduced so far. Their pre- and postconditions are eligibility-aware assertions, that is, assertions that contain occurrences of the eligibility ghost variables. They relate the initial and final states in the eligibility-aware execution of a rule or of a rule program, that is, they reflect the effect of this execution both on the objects in working memory and on the eligibility of rule instances.

Definition 9.5. Let $\mathcal{R}$ be a rule program and $\Delta$ the set of its eligibility ghost variables; let $p, q \in \mathcal{F}(\operatorname{var}(\mathcal{R}), \Delta)$ be two assertions involving eligibility ghost variables. Given a rule $r \in \mathcal{R}$ in the program and its associated eligibility ghost variable $\delta \in \Delta$, the eligibility-aware correctness formula $\{p\} r^{*}\{q\}$ for the rule $r$ holds if:

for any $\mathcal{T}$-compliant states $s$ and $s^{\prime}$, and any valuation $\nu$ well-typed for $r$, such that

one has

$$
s, \nu \models p \quad \text { and } \quad s \stackrel{\left(r^{*}, \nu\right)}{\longrightarrow} s^{\prime}
$$

$$
s^{\prime}, \nu \models q .
$$

Although lexically close to a "regular" correctness formula $\{p\} r\{q\}$, an eligibility-aware one $\{p\} r^{*}\{q\}$ as just defined is more powerful for two reasons. Firstly, it admits occurrences of the eligibility ghost variables in its pre- and postconditions $p$ and $q$. Secondly, it updates these eligibility ghost variables to reflect the effect of the rule execution and thus to ensure consistency with the one-shot eligibility strategy. 
Example. Let us consider again the rule $r_{1}$ used in the previous examples:

$$
\mathrm{r}_{1}\left(\mathrm{p}_{1}: \mathrm{P}\right): \mathrm{p}_{1} \text {.age }>60 \rightarrow \mathrm{p}_{1} \text {.bonus }:=\mathrm{p}_{1} \text {. bonus }+10
$$

and the eligibility-aware assertion proposed earlier, only with a variable renaming:

$$
\varphi \equiv x . \text { bonus }=0 \wedge \neg \delta_{1}(x) \vee x \text {.bonus }=10 \wedge \delta_{1}(x) .
$$

When executing the rule $r_{1}$ on a person $p_{1}$ aged more than 60 from a state that satisfies $\varphi\left(p_{1}\right)$, we can assert that the bonus of that person will finally be 10 :

$$
\left\{\varphi\left(p_{1}\right) \wedge p_{1} \text {.age }>60\right\} r_{1}^{*}\left\{p_{1} \text {.bonus }=10\right\} .
$$

Indeed, either the instance of the rule $r_{1}$ on the object held by $p_{1}$ is eligible, and then the rule instance is selectable and its application increments the bonus from 0 to 10 . Or the rule instance is not eligible and the eligibility-aware execution of the rule instance boils down to a skip, leaving the bonus to its initial value of 10 .

Since the execution of rule programs is inherently eligibility-aware, so are correctness formulas for rule programs. Definition 6.7 of a correctness formula for a rule program still holds when the pre- and postconditions include occurrences of the eligibility ghost variables. A correctness formula $\{p\} \mathcal{R}\{q\}$, where $p, q \in \mathcal{F}(\emptyset, \Delta)$ contain eligibility ghost variables, thus holds if:

for any $\mathcal{T}$-compliant states $s$ and $s^{\prime}$ such that $s \models p$ and $s \stackrel{\mathcal{R}}{\longrightarrow} s^{\prime}$, one has $s^{\prime} \models q$.

Let us remind that, since we consider executions of rule programs under the one-shot execution strategy, partial and total correctness coincide.

We have the following property for the first and last configurations in an execution of a rule program.

Lemma 9.1. Let $\mathcal{R}=\left\{r_{1}, \ldots, r_{n}\right\}$ be a rule program with $r_{i}=\left(\vec{o}_{i}, \vec{T}_{i}, g_{i}, a_{i}\right)$ for $i=1, \ldots, n$, and let $\Delta$ be the set of its eligibility ghost variables; if $s$ and $s^{\prime}$ are two $\mathcal{T}$-compliant states such that $s \stackrel{\mathcal{R}}{\longrightarrow} s^{\prime}$, then

(i) $s \models \bigwedge_{i=1}^{n} \forall \vec{o}_{i}: \vec{T}_{i} \neg \delta_{i}$

(ii) $\quad s^{\prime} \models \bigwedge_{i=1}^{n} \forall \vec{o}_{i}: \vec{T}_{i}\left(\neg g_{i} \vee \delta_{i}\right)$.

Proof. (i) is a direct consequence of the definition of the eligibility ghost variables and of the fact that all rule instances are eligible in the initial configuration of an execution.

(ii) follows from the fact that the execution has ended. Let us note $\left\langle E^{\prime}, s^{\prime}\right\rangle$ the last configuration in an execution of $\mathcal{R}$ from $s$ to $s^{\prime}$. Consider for any $i=1, \ldots, n$ a rule instance $\left(r_{i}, \nu\right)$ well-typed for $\left(\vec{o}_{i}: \vec{T}_{i}\right)$. Since the execution has ended, the rule instance is not applicable or not eligible (or both). If the rule instance is not eligible one has, by definition of the eligibility ghost variables, $s^{\prime}, \nu \models \delta_{i}$. If it is not applicable one has $s^{\prime}, \nu \models \neg g_{i}$. 


\subsection{Interference Freedom}

The present section reviews how the premises of the proof rule introduced in the next section express freedom from the two kinds of interference identified in Section 8.1. In addition, it explains how these premises take eligibility into account, using the eligibility-aware concepts defined in the previous section.

We listed in Section 8.1 two kinds of interference that appear in rule programs: cross-rule interference and interference due to aliasing. The proof rule presented in the previous chapter avoided interference due to aliasing by considering only unary rules, and omitted eligibility. The general proof rule (PR2) introduced in Section 9.3 aims at addressing both kinds of interference, and at taking eligibility into account.

In this section, we discuss how the premises of proof rule (PR2) address interference. Here are these premises:

$$
\begin{aligned}
& \text { for } i=1, \ldots, n: \\
& \qquad\left\{p_{i} \wedge \neg \delta_{i}\right\} r_{i}^{*}\left\{q_{i}\right\} \\
& \text { for } i, j=1, \ldots, n \text { with } i \neq j: \\
& \quad\left\{p_{i} \wedge p_{j} \wedge \neg \delta_{i} \wedge \neg \delta_{j}\right\} r_{j}^{*}\left\{p_{i} \wedge \neg \delta_{i}\right\} \\
& \quad\left\{q_{i} \wedge p_{j} \wedge \delta_{i} \wedge \neg \delta_{j}\right\} r_{j}^{*}\left\{q_{i} \wedge \delta_{i}\right\} \\
& \text { for } i=1, \ldots, n \text { and } \vec{x} \in \operatorname{Var}^{m_{i}}: \\
& \quad\left\{\vec{x} \neq \vec{o}_{i} \wedge p_{i}(\vec{x}) \wedge p_{i} \wedge \neg \delta_{i}(\vec{x}) \wedge \neg \delta_{i}\right\} r_{i}^{*}\left\{p_{i}(\vec{x}) \wedge \neg \delta_{i}(\vec{x})\right\} \\
& \quad\left\{\vec{x} \neq \vec{o}_{i} \wedge q_{i}(\vec{x}) \wedge p_{i} \wedge \delta_{i}(\vec{x}) \wedge \neg \delta_{i}\right\} r_{i}^{*}\left\{q_{i}(\vec{x}) \wedge \delta_{i}(\vec{x})\right\} .
\end{aligned}
$$

Using eligibility-aware correctness formulas allows these premises to take eligibility into account. The second and third group of correctness formulas express interference freedom.

\subsubsection{Cross-Rule Interference}

The correctness formulas expressing cross-rule interference freedom in proof rule (PR1) are

$$
\left.\begin{array}{l}
\left\{p_{i} \wedge p_{j}\right\} r_{j}\left\{p_{i}\right\} \\
\left\{q_{i} \wedge p_{j}\right\} r_{j}\left\{q_{i}\right\}
\end{array}\right\} \text { for } i, j=1, \ldots, n \text { with } i \neq j .
$$

The correctness formula $\left\{p_{i} \wedge p_{j}\right\} r_{j}\left\{p_{i}\right\}$, for example, states that the assertion $p_{i}$ is preserved by an execution of the rule $r_{j}$. The restriction to executions from a state where $p_{j}$ holds can be justified as a consequence of Lemma 8.3. Indeed, for an instance of the rule $r_{j}$ to be executed, it must be eligible. And since the configurations of the abstract machine during the execution of the rule program are consistent with the correctness formulas focused on the rules, the precondition $p_{j}$ associated with the rule $r_{j}$ then holds.

However the fact that $p_{j}$ holds is only a consequence of the eligibility of the instance of $r_{j}$. Explicitly taking eligibility into account requires that $\neg \delta_{j}$ holds as well.

Similarly, the soundness of proof rule (PR2) does not require the rule $r_{j}$ to be interference free with the entire assertion $p_{i}$, but only with the portion of this assertion that holds when the rule $r_{i}$ is eligible. Hence the eligibility-aware correctness formula found in the premises of this proof rule, which states that 
the assertion $p_{i} \wedge \neg \delta_{i}$ is preserved by an eligibility-aware execution of the rule $r_{j}$ from a state where $p_{j} \wedge \neg \delta_{j}$ holds.

One might notice that the pre- and postconditions of eligibility-aware correctness formulas are typically longer than those which do not contain eligibility ghost variables. Indeed, a correctness formula for a rule $r_{i}$ usually addresses cross-rule interference by including the eligibility ghost variables for the other rules. It is typically of the following form, assuming a three-rule program:

$$
p_{1} \equiv p_{1}^{00} \wedge \neg \delta_{2} \wedge \neg \delta_{3} \vee p_{1}^{01} \wedge \neg \delta_{2} \wedge \delta_{3} \vee p_{1}^{10} \wedge \delta_{2} \wedge \neg \delta_{3} \vee p_{1}^{11} \wedge \delta_{2} \wedge \delta_{3} .
$$

where the $p_{1}^{x y}$ are assertions in the variables of $r_{1}$, containing no ghost variables.

As a result, a conjunction with an eligibility ghost variable has the effect of "selecting" disjuncts in the assertion. For example,

$$
p_{1} \wedge \neg \delta_{2} \quad \text { is } \quad\left(p_{1}^{00} \wedge \neg \delta_{3} \vee p_{1}^{01} \wedge \delta_{3}\right) \wedge \neg \delta_{2} .
$$

This phenomenon is exploited when devising the pre- and postconditions of the correctness formulas for the individual rules, so that they take into account the past executions of the other rules.

\subsubsection{Interference due to Aliasing}

Interference due to aliasing, defined in Section 8.1.2, occurs when the execution of a rule instance does not preserve the pre- or postcondition of a correctness formula on a partially distinct tuple of objects.

This kind of interference was avoided in the previous chapter because we considered only rule programs made of unary rules, which prevented aliasing altogether by the fact that formulas are flat, as discussed in Section 8.2. As a consequence, an instance of a rule could not affect the truth values of preand postconditions considered on other objects. This property is formalized by Lemmas 8.1 and 8.4.

When rules with an arbitrary number of variables are considered, the objects in various instances of a rule are no longer guaranteed to be distinct. In particular, Lemmas 8.1 and 8.4 do not extend to the n-ary case. As a result, the premises of a proof rule suited to the n-ary case, such as (PR2), must express that executing a rule instance preserves the pre- and postconditions of the correctness formulas for all possible mappings of variables to objects.

The second group of correctness formulas in the premises of (PR2), discussed in Section 9.2.1, involves variables of two rules. For example, the correctness formula $\left\{p_{i} \wedge p_{j} \wedge \neg \delta_{i} \wedge \neg \delta_{j}\right\} r_{j}^{*}\left\{p_{i} \wedge \neg \delta_{i}\right\}$ implicitly refers to the variables $\vec{o}_{i}=$ $\left(o_{1}^{i}, \ldots, o_{m_{i}}^{i}\right)$ when mentioning $p_{i}$ and $\delta_{i}$, and to the variables $\vec{o}_{j}=\left(o_{1}^{j}, \ldots, o_{m_{j}}^{j}\right)$ when mentioning $p_{j}, \delta_{j}$, and $r_{j}$. All cases of equality or disequality between these variables are covered in these correctness formulas.

The missing cases are for two instances of the same rule, since the above correctness formulas are considered for $i \neq j$. These cases are addressed by the third group of correctness formulas in the premises of (PR2). In these correctness formulas, two instances of a rule $r_{i}$ are considered by providing explicit variable tuples; the instances are made distinct by additionally requiring the variable tuples to be different. Hence a correctness formula such as

$$
\left\{\vec{x} \neq \vec{o}_{i} \wedge p_{i}(\vec{x}) \wedge p_{i} \wedge \neg \delta_{i}(\vec{x}) \wedge \neg \delta_{i}\right\} r_{i}^{*}\left\{p_{i}(\vec{x}) \wedge \neg \delta_{i}(\vec{x})\right\} .
$$




\subsection{General Proof Rule}

The proof rule for programs made of rules of arbitrary arities, and correctness formulas that take eligibility into account, is given in this section. Its soundness is proven, based on the fact that at each step in the execution of a rule program, the truth value of all pre- and postconditions of individual correctness formulas is left unchanged, except for the rule instance applied.

The proof rule is not only sound, but also relatively complete, in the sense that it can prove any valid correctness formula on a rule program, provided the assertions from first-order logic and from the theory fixed in Section 3.1 can be proven. This property is exposed in the next section.

With the introduction of eligibility ghost variables and the consideration of interference due to aliasing, we have gathered the material for the proof rule at the heart of our verification method. It is given in Theorem 9.2 below.

Theorem 9.2. Let $\mathcal{R}=\left\{r_{1}, \ldots, r_{n}\right\}$ be a rule program and $\Delta$ the set of its eligibility ghost variables. For $i=1, \ldots, n$, let us note $r_{i}=\left(\vec{o}_{i}, \vec{T}_{i}, g_{i}, a_{i}\right)$ with $m_{i}=\left|r_{i}\right|$, and let $p_{i}, q_{i} \in \mathcal{F}_{\Delta}\left(r_{i}\right)$ be eligibility-aware assertions focused on the rule $r_{i}$. The proof rule (PR2) below is sound.

$$
\begin{aligned}
& \text { for } i=1, \ldots, n: \\
& \quad\left\{p_{i} \wedge \neg \delta_{i}\right\} r_{i}^{*}\left\{q_{i}\right\} \\
& \text { for } i, j=1, \ldots, n \text { with } i \neq j: \\
& \quad\left\{p_{i} \wedge p_{j} \wedge \neg \delta_{i} \wedge \neg \delta_{j}\right\} r_{j}^{*}\left\{p_{i} \wedge \neg \delta_{i}\right\} \\
& \quad\left\{q_{i} \wedge p_{j} \wedge \delta_{i} \wedge \neg \delta_{j}\right\} r_{j}^{*}\left\{q_{i} \wedge \delta_{i}\right\} \\
& \text { for } i=1, \ldots, n \text { and } \vec{x} \in \operatorname{Var}^{m_{i}}: \\
& \quad\left\{\vec{x} \neq \vec{o}_{i} \wedge p_{i}(\vec{x}) \wedge p_{i} \wedge \neg \delta_{i}(\vec{x}) \wedge \neg \delta_{i}\right\} r_{i}^{*}\left\{p_{i}(\vec{x}) \wedge \neg \delta_{i}(\vec{x})\right\} \\
& \quad\left\{\vec{x} \neq \vec{o}_{i} \wedge q_{i}(\vec{x}) \wedge p_{i} \wedge \delta_{i}(\vec{x}) \wedge \neg \delta_{i}\right\} r_{i}^{*}\left\{q_{i}(\vec{x}) \wedge \delta_{i}(\vec{x})\right\} \\
& \hline\left\{\bigwedge_{i=1}^{n} \forall \vec{o}_{i}: \vec{T}_{i}\left(p_{i} \wedge \neg \delta_{i}\right)\right\} \mathcal{R}\left\{\bigwedge_{i=1}^{n} \forall \vec{o}_{i}: \vec{T}_{i}\left(q_{i} \wedge\left(\delta_{i} \vee \neg g_{i}\right)\right)\right\}
\end{aligned}
$$

The premises of proof rule (PR2) have just been discussed in Section 9.2. Its conclusion might seem quite complex, as it includes combinations of the $p_{i}, q_{i}$ assertions and the eligibility ghost variables, and additionally involves the rule guards in its postcondition. As for the premises of the proof rule, the presence of eligibility ghost variables in the pre- and postconditions of the conclusion is actually a tool. Including them selects the disjuncts in the $p_{i}, q_{i}$ assertions that are relevant in the first and last configurations of an execution of the rule program. The rule guards in the postcondition enhances the completeness of the proof rule, notably in the case where the precondition does not imply the rule guards. This is related to Lemma 9.1, and is discussed in Section 9.5 on an example.

The proof of Theorem 9.2 follows a path similar to the proof of Theorem 8.2 in Section 8.3. We start by defining what it means for a state to be consistent with the eligibility-aware correctness formulas focused on the rules. We then show that consistency is an invariant of a rule program execution. Finally we leverage this result to show the soundness of proof rule (PR2). 
Definition 9.6. Given a rule program $\mathcal{R}=\left\{r_{1}, \ldots, r_{n}\right\}$, the set of its eligibility ghost variables $\Delta=\left\{\delta_{1}, \ldots, \delta_{n}\right\}$, and eligibility-aware correctness formulas $\left\{p_{i}\right\} r_{i}^{*}\left\{q_{i}\right\}$ for $i=1, \ldots, n$ focused on the rules of $\mathcal{R}$, a $\mathcal{T}$-compliant state $s$ is consistent with the correctness formulas, or $s$ is cf-consistent, if

$$
s \models \bigwedge_{i=1}^{n} \forall \vec{o}_{i}: \vec{T}_{i}\left(\neg \delta_{i} \wedge p_{i} \vee \delta_{i} \wedge q_{i}\right) .
$$

We can now state Lemma 9.3, by which the premises of proof rule (PR2) ensure that an execution of a rule program that starts in a cf-consistent state results in a cf-consistent state.

Lemma 9.3. Let $\mathcal{R}=\left\{r_{1}, \ldots, r_{n}\right\}$ be a rule program and $\Delta$ the set of its eligibility ghost variables. For $i=1, \ldots, n$, let us note $r_{i}=\left(\vec{o}_{i}, \vec{T}_{i}, g_{i}, a_{i}\right)$ with $m_{i}=\left|r_{i}\right|$, and let $p_{i}, q_{i} \in \mathcal{F}_{\Delta}\left(r_{i}\right)$ be eligibility-aware assertions focused on the rule $r_{i}$, such that

$$
\begin{aligned}
& \text { for } i=1, \ldots, n: \\
& \quad\left\{p_{i} \wedge \neg \delta_{i}\right\} r_{i}^{*}\left\{q_{i}\right\} \\
& \text { for } i, j=1, \ldots, n \text { with } i \neq j: \\
& \quad\left\{p_{i} \wedge p_{j} \wedge \neg \delta_{i} \wedge \neg \delta_{j}\right\} r_{j}^{*}\left\{p_{i} \wedge \neg \delta_{i}\right\} \\
& \quad\left\{q_{i} \wedge p_{j} \wedge \delta_{i} \wedge \neg \delta_{j}\right\} r_{j}^{*}\left\{q_{i} \wedge \delta_{i}\right\} \\
& \text { for } i=1, \ldots, n \text { and } \vec{x} \in \operatorname{Var}^{m_{i}}: \\
& \quad\left\{\vec{x} \neq \vec{o}_{i} \wedge p_{i}(\vec{x}) \wedge p_{i} \wedge \neg \delta_{i}(\vec{x}) \wedge \neg \delta_{i}\right\} r_{i}^{*}\left\{p_{i}(\vec{x}) \wedge \neg \delta_{i}(\vec{x})\right\} \\
& \quad\left\{\vec{x} \neq \vec{o}_{i} \wedge q_{i}(\vec{x}) \wedge p_{i} \wedge \delta_{i}(\vec{x}) \wedge \neg \delta_{i}\right\} r_{i}^{*}\left\{q_{i}(\vec{x}) \wedge \delta_{i}(\vec{x})\right\} .
\end{aligned}
$$

Given two $\mathcal{T}$-compliant states $s$ and $s^{\prime}$ such that $s \stackrel{\mathcal{R}}{\longrightarrow} s^{\prime}$, if $s$ is consistent with the correctness formulas $\left\{p_{i}\right\} r_{i}^{*}\left\{q_{i}\right\}$, then $s^{\prime}$ is as well.

Proof. The proof goes by induction on the length of the executions of $\mathcal{R}$.

Base step. If the execution is empty then $s^{\prime}=s$ and the lemma holds by hypothesis.

Induction step. We consider an execution $\left\langle E_{0}, s_{0}\right\rangle \stackrel{\mathcal{R}}{\longrightarrow}\left\langle E_{k}, s_{k}\right\rangle$ of length $k \geq 1$ of the rule program, such that $s_{0}$ is cf-consistent, and we assume that $s_{k-1}$ is cf-consistent.

Let us note $\left\langle E_{k-1}, s_{k-1}\right\rangle \stackrel{\left(r_{i_{k}}, \vec{O}_{k}\right)}{\longrightarrow}\left\langle E_{k}, s_{k}\right\rangle$ the last transition of this execution, where $i_{k} \in\{1, \ldots, n\}$ and $\vec{O}_{k}=\left(O_{1}^{k}, \ldots, O_{m_{i_{k}}}^{k}\right) \in \mathcal{M}^{\left(r_{i_{k}}\right)}$ with $m_{i_{k}}=\left|r_{i_{k}}\right|$. By the transition rule (9.1) we have

$$
\left\langle s_{k-1}, s_{k}\right\rangle, \nu_{k} \models \neg \delta_{i_{k}} \wedge g_{i_{k}} \wedge \hat{a}_{i_{k}} \wedge \hat{\delta}_{i_{k}}
$$

for any valuation $\nu_{k}$ such that $\nu_{k}\left(o_{j}^{i_{k}}\right)=O_{j}^{k}$ for $j=1, \ldots, m_{i_{k}}$.

We want to prove that $s_{k} \models \bigwedge_{i=1}^{n} \forall \vec{o}_{i}: \vec{T}_{i}\left(\neg \delta_{i} \wedge p_{i} \vee \delta_{i} \wedge q_{i}\right)$, that is, that for any $i \in\{1, \ldots, n\}$ and any valuation $\nu$ well-typed for $\left(\vec{o}_{i}: \vec{T}_{i}\right)$ one has $s_{k}, \nu \models$ $\neg \delta_{i} \wedge p_{i} \vee \delta_{i} \wedge q_{i}$. Let us thus consider some $i \in\{1, \ldots, n\}$ and some valuation $\nu$ well-typed for $\left(\vec{o}_{i}: \vec{T}_{i}\right)$.

The induction hypothesis states that $s_{k-1} \models \bigwedge_{i=1}^{n} \forall \vec{o}_{i}: \vec{T}_{i}\left(\neg \delta_{i} \wedge p_{i} \vee \delta_{i} \wedge q_{i}\right)$, which gives in particular that either $s_{k-1}, \nu \models \neg \delta_{i} \wedge p_{i}$ or $s_{k-1}, \nu \models \delta_{i} \wedge q_{i}$ holds. Therefore, we have

$$
s_{k-1}, \nu \models \varphi
$$


where $\varphi$ denotes either $\neg \delta_{i} \wedge p_{i}$ or $\delta_{i} \wedge q_{i}$.

We now examine in turn the cases where the rule instance $R=\left(r_{i}, \nu\right)$ is equal to the rule instance $R_{k}=\left(r_{i_{k}}, \vec{O}_{k}\right)$ used in the last transition, or not; and if not, why.

- First case: $R=R_{k}$, that is, $R_{k}=\left(r_{i}, \nu\right)$.

Since $i=i_{k}$ we have $\nu\left(o_{j}^{i_{k}}\right)=\nu\left(o_{j}^{i}\right)=O_{j}^{k}$ for $j=1, \ldots, m_{i_{k}}$. The valuation $\nu$ thus qualifies for (d), which gives in particular $s_{k-1}, \nu \models \neg \delta_{i}$. By induction hypothesis this gives $s_{k-1}, \nu \models p_{i} \wedge \neg \delta_{i}$, and hence by (a) we have $s_{k}, \nu \models q_{i}$.

By (d) again, we have $s_{k}, \nu \models \delta_{i}$, which with the previous gives

$$
s_{k}, \nu \models \delta_{i} \wedge q_{i} .
$$

- Second case: $R \neq R_{k}$ but still $i=i_{k}$. Then there must exists some $\ell \in$ $\left\{1, \ldots, m_{i}\right\}$ such that $\nu\left(o_{\ell}^{i}\right) \neq O_{\ell}^{k}$.

Let us consider a tuple of variables $\vec{x} \in \operatorname{Var}^{m_{i}}$ and a valuation $\nu^{\prime}$, such that for $j=1, \ldots, m_{i}$ one has $\nu^{\prime}\left(o_{j}^{i}\right)=O_{j}^{k}$ and $\nu^{\prime}\left(x_{j}\right)=\nu\left(o_{j}^{i}\right)$.

Since $\nu^{\prime}\left(x_{\ell}\right)=\nu\left(o_{\ell}^{i}\right) \neq O_{\ell}^{k}=\nu^{\prime}\left(o_{\ell}^{i}\right)$, we have

$$
\nu^{\prime} \models \vec{x} \neq \vec{o}_{i} .
$$

Also, since $\nu^{\prime}\left(o_{j}^{i}\right)=O_{j}^{k}$ for $j=1, \ldots, m_{i}$ we have

$$
\left(r_{i}, \nu^{\prime}\right)=R_{k}
$$

By Lemma 4.2 we also have from (e) that

$$
s_{k-1}, \nu^{\prime} \models \varphi(\vec{x}) .
$$

In addition, since $i=i_{k}$ the valuation $\nu^{\prime}$ qualifies for (d) and we thus have

$$
s_{k-1}, \nu^{\prime} \models \neg \delta_{i}
$$

which by induction hypothesis gives

$$
s_{k-1}, \nu^{\prime} \models p_{i} .
$$

From (f), (h), (i), and (j) we have $s_{k-1}, \nu^{\prime} \models \vec{x} \neq \vec{o}_{i} \wedge \varphi(\vec{x}) \wedge \neg \delta_{i} \wedge p_{i}$. By (g) and the interference freedom correctness formulas (c) this gives $s_{k}, \nu^{\prime} \models \varphi(\vec{x})$, which by Lemma 4.2 finally gives

$$
s_{k}, \nu \models \varphi .
$$

- Third case: $R \neq R_{k}$ with $i \neq i_{k}$

Let $\nu^{\prime}$ be a valuation such that for $j=1, \ldots, m_{i_{k}}$ one has $\nu^{\prime}\left(o_{j}^{i_{k}}\right)=O_{j}^{k}$ and for $j=1, \ldots, m_{i}$ one has $\nu^{\prime}\left(o_{j}^{i}\right)=\nu\left(o_{j}^{i}\right)$. We have

$$
\left(r_{i_{k}}, \nu^{\prime}\right)=R_{k} .
$$

By Lemma 4.1 we also have from (e) that

$$
s_{k-1}, \nu^{\prime} \models \varphi .
$$

In addition, the valuation $\nu^{\prime}$ qualifies for (d) and we thus have

$$
s_{k-1}, \nu^{\prime} \models \neg \delta_{i}
$$


which by induction hypothesis gives

$$
s_{k-1}, \nu^{\prime} \models p_{i} .
$$

From (l), (m), and (n) we have $s_{k-1}, \nu^{\prime} \models \varphi \wedge \neg \delta_{i} \wedge p_{i}$. By (k) and the interference freedom correctness formulas (b) this gives $s_{k}, \nu^{\prime} \models \varphi$, which by Lemma 4.1 finally gives

$$
s_{k}, \nu \models \varphi .
$$

- Summary. We have considered an arbitrary $i \in\{1, \ldots, n\}$ and an arbitrary valuation $\nu$, such that $\nu\left(o_{j}^{i}\right) \in \mathcal{M}\left(T_{j}^{i}\right)$ for $j=1, \ldots, m_{i}$.

In the first case, we have shown that $s_{k}, \nu \models \delta_{i} \wedge q_{i}$.

In the second and third cases, we have shown that $s_{k}, \nu \models \varphi$, where $\varphi \equiv$ $\neg \delta_{i} \wedge p_{i}$ or $\varphi \equiv \delta_{i} \wedge q_{i}$.

In all cases we have thus shown that $s_{k}, \nu \models \neg \delta_{i} \wedge p_{i} \vee \delta_{i} \wedge q_{i}$. That is, we have shown that $s_{k}$ is cf-consistent.

We now turn to the proof of Theorem 9.2.

Proof of Theorem 9.2. Let $\mathcal{R}=\left\{r_{1}, \ldots, r_{n}\right\}$ be a rule program and $\Delta$ the set of its eligibility ghost variables. For $i=1, \ldots, n$, let us note $r_{i}=\left(\vec{o}_{i}, \vec{T}_{i}, g_{i}, a_{i}\right)$ with $m_{i}=\left|r_{i}\right|$, and let $p_{i}, q_{i} \in \mathcal{F}_{\Delta}\left(r_{i}\right)$ be eligibility-aware assertions focused on the rule $r_{i}$, such that the premises of (PR2) hold, and among them

$$
\left\{p_{i} \wedge \neg \delta_{i}\right\} r_{i}^{*}\left\{q_{i}\right\}
$$

Consider two $\mathcal{T}$-compliant states $s$ and $s^{\prime}$, such that $s \stackrel{\mathcal{R}}{\longrightarrow} s^{\prime}$ and

$$
s \models \bigwedge_{i=1}^{n} \forall \vec{o}_{i}: \vec{T}_{i}\left(p_{i} \wedge \neg \delta_{i}\right)
$$

We want to prove the postcondition of the correctness formula in the conclusion of the proof rule, that is, $s \models \bigwedge_{i=1}^{n} \forall \vec{o}_{i}: \vec{T}_{i}\left(q_{i} \wedge\left(\delta_{i} \vee \neg g_{i}\right)\right)$. Hypothesis (b) gives that $s$ is cf-consistent. Therefore, by Lemma 9.3, we have that $s^{\prime}$ is cf-consistent, that is,

$$
s^{\prime} \models \bigwedge_{i=1}^{n} \forall \vec{o}_{i}: \vec{T}_{i}\left(p_{i} \wedge \neg \delta_{i} \vee q_{i} \wedge \delta_{i}\right) .
$$

Let us consider some $i \in\{1, \ldots, n\}$ and some valuation $\nu$ well-typed for $\left(\vec{o}_{i}: \vec{T}_{i}\right)$. The above means that we have

$$
s^{\prime}, \nu \models p_{i} \wedge \neg \delta_{i} \vee q_{i} \wedge \delta_{i} .
$$

That is:

(i) Either $s^{\prime}, \nu \models q_{i} \wedge \delta_{i}$, which implies

$$
s^{\prime}, \nu \models q_{i} \wedge\left(\delta_{i} \vee \neg g_{i}\right) .
$$

(ii) $\mathrm{Or}$

$$
s^{\prime}, \nu \models p_{i} \wedge \neg \delta_{i} .
$$


Since $s^{\prime}$ is the final state of the execution of $\mathcal{R}$, the rule instance $\left(r_{i}^{*}, \nu\right)$ is not selectable, that is by Definition 9.2, $s^{\prime}, \nu \models \neg g_{i} \vee \delta_{i}$. With (c) this gives

$$
s^{\prime}, \nu \models \neg g_{i} .
$$

As the rule instance $\left(r_{i}^{*}, \nu\right)$ is not selectable, we also have $s^{\prime} \stackrel{\left(r_{i}^{*}, \nu\right)}{\longrightarrow} s^{\prime}$. Therefore, from (c) and (a), we have

$$
s^{\prime}, \nu \models q_{i} .
$$

This together with (d) gives $s^{\prime}, \nu \models q_{i} \wedge \neg g_{i}$, which implies

$$
s^{\prime}, \nu \models q_{i} \wedge\left(\delta_{i} \vee \neg g_{i}\right) .
$$

In all cases, and for any $i \in\{1, \ldots, n\}$ and valuation $\nu$ well-typed for $\left(\vec{o}_{i}: \vec{T}_{i}\right)$, we have $s^{\prime}, \nu \models q_{i} \wedge\left(\delta_{i} \vee \neg g_{i}\right)$. This means that we have the expected

$$
s \models \bigwedge_{i=1}^{n} \forall \vec{o}_{i}: \vec{T}_{i}\left(q_{i} \wedge\left(\delta_{i} \vee \neg g_{i}\right)\right)
$$

which proves the correctness formula in the conclusion of proof rule (PR2), and proves the theorem.

\subsection{Relative Completeness}

The proof rule introduced in the previous section is relatively complete, which means that it can prove any valid correctness formula on a rule program, relatively to the existence of complete proof rules for the implications used in the side conditions of the (CONSEQUENCE) proof rule.

The proof of the relative completeness of our proof rule is based on a reduction of a rule program to the nondeterministic program derived from the rule program, as described in Section 7.3.2, and on the relative completeness of the (DO-OD) proof rule given in the same section.

The proof system proposed by our verification method is built on proof rule (PR2). It also includes the (CONSEQUENCE) proof rule (see Section 6.1.2), and the proof rules inherited from the theory $\Theta$ fixed in Section 3.1. The question of whether this proof system is complete has to be addressed from each of these three viewpoints.

The (CONSEQUENCE) proof rule uses implications between assertions in its side conditions. Thus, the completeness of our proof system depends on the provability of these implications. The assertions involved in these implications, as well as in the pre- and postconditions of correctness formulas, are relative to the theory $\Theta$. Therefore, their provability is conditioned by the completeness of the proof rules inherited from $\Theta$.

As a result, we are interested in the classical notion of relative completeness [Coo78]. That is, we focus on the completeness of proof rule (PR2) relatively to the existence of a complete proof system (or of an oracle) for the validity of the implications used in the side conditions of the (CONSEQUENCE) proof rule. 
Theorem 9.4. The proof rule (PR2), and our verification method for rule programs, are relatively complete. That is, given a rule program $\mathcal{R}=\left\{r_{1}, \ldots, r_{n}\right\}$ and a correctness formula $\{p\} \mathcal{R}\{q\}$ for this program, if $\{p\} \mathcal{R}\{q\}$ is valid then there exist eligibility-aware assertions $p_{1}, q_{1} \in \mathcal{F}_{\Delta}\left(r_{1}\right), \ldots, p_{n}, q_{n} \in \mathcal{F}_{\Delta}\left(r_{n}\right)$ focused on the rules in the program, such that the individual correctness formulas $\left\{p_{i} \wedge \neg \delta_{i}\right\} r_{i}^{*}\left\{q_{i}\right\}$ are valid, are interference free, and such that the correctness formula $\{p\} \mathcal{R}\{q\}$ can be derived by (PR2), under the assumption that the implications used in the side conditions of the (CONSEQUENCE) proof rule can be proven.

We have shown in Section 7.3.2 that rule programs can be reduced to nondeterministic program. That is, from any rule program, a nondeterministic program can be derived that satisfies the same correctness formulas. The proof of the relative completeness of (PR2) is based on this equivalence in behavior between rule programs and derived nondeterministic programs, and on the relative completeness of the (DO-OD) proof rule [Apt84].

We start this section with a few preliminaries about nondeterministic programs that will be of use in the proof of Theorem 9.4. Then we proceed to the proof.

\section{Preliminaries: Nondeterministic Programs}

Section 7.3.1 introduces nondeterministic programs based on Dijkstra's do-od statement [Dij75]. Section 7.3.2 defines the nondeterministic program derived from a rule program and shows that both satisfy the same correctness formulas.

Let us remind the definition of the nondeterministic program $P_{\mathcal{R}}$ derived from a rule program $\mathcal{R}=\left\{r_{1}, \ldots, r_{n}\right\}$ with $r_{i}=\left(\vec{o}_{i}, \vec{T}_{i}, g_{i}, a_{i}\right)$ for $i=1, \ldots, n$ :

$$
P_{\mathcal{R}}(\vec{o}: \vec{T}) \stackrel{\text { def }}{=} \pi_{1}:=\text { false } ; \ldots ; \pi_{n}:=\text { false } ; \text { do } \square_{i=1}^{n} g_{i} \wedge \neg \pi_{i} \neg a_{i} ; \pi_{i}:=\text { true od. }
$$

The program $P_{\mathcal{R}}$ is a nondeterministic program in the variables $o_{1}^{1}, \ldots, o_{m_{n}}^{n}$. The notation $(\vec{o}: \vec{T})$ is a shorthand for $\left(\vec{o}_{1}: \vec{T}_{1}\right) \ldots\left(\vec{o}_{n}: \vec{T}_{n}\right)$.

In addition, $P_{\mathcal{R}}$ contains as many ghost variables as there are rules in $\mathcal{R}$. To avoid confusion with eligibility ghost variables introduced earlier for rule programs, we name them $\pi_{i}$ in $P_{\mathcal{R}}$. We will refer to them collectively as the set $\Pi=\left\{\pi_{1}, \ldots, \pi_{n}\right\} \subset$ Ghost. Note that although we refrain from identifying $\pi_{i}$ and $\delta_{i}$, both sets of ghost variables intuitively encode the same concept, namely whether an instance of the corresponding rule has been executed.

The program $P_{\mathcal{R}}$ is a sequence of assignments and a nondeterministic loop. We materialize this by noting $P_{\mathcal{R}} \equiv H_{\Pi} ; P_{\mathcal{R}}^{\Pi}$ with:

$$
\begin{aligned}
& H_{\Pi}(\vec{o}: \vec{T}) \equiv \pi_{1}:=\text { false } ; \ldots ; \pi_{n}:=\text { false } \\
& P_{\mathcal{R}}^{\Pi}(\vec{o}: \vec{T}) \equiv \text { do } g_{1} \wedge \neg \pi_{1} \neg a_{1} ; \pi_{1}:=\text { true }\left[\ldots \square g_{n} \wedge \neg \pi_{n} \neg a_{n} ; \pi_{n}:=\right.\text { true od }
\end{aligned}
$$

Let us also remind that this program is to be executed on a working memory under the saturated semantics (described in Section 7.3.1). That is, in an execution of $P_{\mathcal{R}}$ on a working memory $\mathcal{M}$ :

(i) Each assignment $\pi_{i}:=$ false in $H_{\Pi}$ is instantiated and executed for all the possible mappings of $\vec{o}_{i}$ onto object tuples from $\mathcal{M}^{\left(r_{i}\right)}$; 
(ii) In the execution of the loop in $P_{\mathcal{R}}^{\Pi}$, each guarded command is instantiated and assessed for all these mappings as well. On each loop iteration, one instance of one guarded command is selected and executed.

Assigning a value to a ghost variable $\pi_{i}$, whether in $H_{\Pi}$ or in the action part of a guarded command, is done after the assignment has been instantiated on a tuple of objects $\vec{O} \in \mathcal{M}^{\left(r_{i}\right)}$. As described in Section 7.2.1, neither the value of the ghost variable on other object tuples nor the values of the other ghost variables are changed in the state that results from such an assignment. Let us note $s$ and $s^{\prime}$ the initial and final states of an assignment to the ghost variable $\pi_{i}$ with the value $b$ under a valuation $\nu$ that maps the variables in $\vec{o}_{i}$ onto the objects in $\vec{O}$. The assignment is then described by $\left\langle s, s^{\prime}\right\rangle, \nu \models \hat{\pi}_{i}(b)$, with the transition assertion $\hat{\pi}_{i}(b)$ defined as follows:

$$
\hat{\pi}_{i}(b) \stackrel{\text { def }}{=} \pi_{i}^{\prime}\left(\vec{o}_{i}\right)=b \wedge \forall \vec{o}\left(\vec{o} \neq \vec{o}_{i} \rightarrow \pi_{i}^{\prime}(\vec{o})=\pi_{i}(\vec{o})\right) \wedge \bigwedge_{\pi \in \Pi \backslash\left\{\pi_{i}\right\}} \forall \vec{o}\left(\pi^{\prime}(\vec{o})=\pi(\vec{o})\right) .
$$

It is no surprise that $\hat{\pi}_{i}$ (true) is identical to $\hat{\delta}_{i}$ introduced by Definition 9.3, modulo the ghost variable names.

Finally, the decomposition of $P_{\mathcal{R}}$ into $H_{\Pi} ; P_{\mathcal{R}}^{\Pi}$ leads to the following lemma.

Lemma 9.5. Let $\mathcal{R}$ be a rule program and $P_{\mathcal{R}} \equiv H_{\Pi} ; P_{\mathcal{R}}^{\Pi}$ the nondeterministic program derived from it. Given two global assertions $p, q \in \mathcal{F}(\emptyset, \emptyset)$ containing no ghost variable,

$$
\text { if } \quad\{p\} P_{\mathcal{R}}\{q\} \quad \text { then } \quad\left\{p \wedge \bigwedge_{i=1}^{n} \forall \vec{o}_{i}: \vec{T}_{i} \neg \pi_{i}\right\} P_{\mathcal{R}}^{\Pi}\{q\} .
$$

Proof. Assume that $\{p\} P_{\mathcal{R}}\{q\}$ holds, and consider two $\mathcal{T}$-compliant states $s$ and $s^{\prime}$, such that $s=p \wedge \bigwedge_{i=1}^{n} \forall \vec{o}_{i}: \vec{T}_{i} \neg \pi_{i}$ and $s \stackrel{P_{\mathcal{R}}^{\Pi}}{\longrightarrow} s^{\prime}$.

Let $s^{\natural}$ be the ghost-independent state corresponding to $s$, that is by Definition 7.3, the state that interprets all symbols in the same way as $s$, except ghost variables which are all interpreted by the constant function returning $\perp$. Since we have in particular $s \models p$ and since $p$ contains no ghost variable, we also have $s^{\natural} \models p$.

On the other hand, an execution of $H_{\Delta}$ from $s^{\natural}$ has the (only) effect of setting all the ghost variables $\pi_{i}$ to false. The resulting state is therefore $s$. That is, $s^{\natural} \stackrel{H_{\Delta}}{\longrightarrow} s$ and hence $s^{\natural} \stackrel{P_{\mathcal{R}}}{\longrightarrow} s^{\prime}$.

By $\{p\} P_{\mathcal{R}}\{q\}$ we finally have $s^{\prime} \models q$, which establishes the targeted correctness formula.

\section{Proof of Relative Completeness}

As mentioned earlier, the proof of the relative completeness of (PR2) is based both on the relative completeness of the (DO-OD) proof rule and on the fact that a rule program and the nondeterministic program derived from it satisfy the same correctness formulas.

The proof goes through the following steps. First, we establish the existence of a proof of the correctness formula for the nondeterministic program derived from the rule program. From this proof we synthesize assertions focused on the 
rules. Then we show that these assertions form valid eligibility-aware correctness formulas on the rules and that these correctness formulas are interference free, for both forms of interference. We conclude the proof by showing that the global correctness formula derived from these individual correctness formulas leads to the correctness formula to prove.

Proof of Theorem 9.4. Consider a rule program $\mathcal{R}=\left\{r_{1}, \ldots, r_{n}\right\}$ with $r_{i}=$ $\left(\vec{o}_{i}, \vec{T}_{i}, g_{i}, a_{i}\right)$ for $i=1, \ldots, n$, and two global assertions $p, q \in \mathcal{F}(\emptyset, \emptyset)$, such that the correctness formulas $\{p\} \mathcal{R}\{q\}$ holds.

Our objective here is to show that there exists a proof of $\{p\} \mathcal{R}\{q\}$ by proof rule (PR2). That is, that there exist $2 n$ eligibility-aware assertions $p_{i}, q_{i} \in \mathcal{F}_{\Delta}\left(r_{i}\right)$ focused on the rules $r_{1}, \ldots, r_{n}$, such that the individual correctness formulas $\left\{p_{i} \wedge \neg \delta_{i}\right\} r_{i}^{*}\left\{q_{i}\right\}$ hold, are interference free, and the correctness formula derived from them by (PR2) leads to $\{p\} \mathcal{R}\{q\}$.

To this end, we leverage the relative completeness of the (DO-OD) proof rule to exhibit assertions focused on the rules, and we show that these assertions satisfy the expected criteria.

- Existence of the assertions. Let us consider the nondeterministic program $P_{\mathcal{R}}$ derived from the rule program $\mathcal{R}:$

$$
P_{\mathcal{R}}(\vec{o}: \vec{T}) \equiv \pi_{1}:=\text { false } ; \ldots ; \pi_{n}:=\text { false } ; \text { do } \square_{i=1}^{n} g_{i} \wedge \neg \pi_{i} \neg a_{i} ; \pi_{i}:=\text { true od. }
$$

Theorem 7.5 establishes that $P_{\mathcal{R}}$ satisfies the same correctness formulas as $\mathcal{R}$. That is, the correctness formula $\{p\} P_{\mathcal{R}}\{q\}$ holds. By Lemma 9.5 the following correctness formula thus holds:

$$
\left\{p \wedge \bigwedge_{i=1}^{n} \forall \vec{o}_{i}: \vec{T}_{i} \neg \pi_{i}\right\} \text { do } \square_{i=1}^{n} g_{i} \wedge \neg \pi_{i} \neg a_{i} ; \pi_{i}:=\text { true od }\{q\}
$$

The (DO-OD) proof rule is given in Section 7.3.2 and reproduced below:

$$
\begin{gathered}
\left\{I \wedge B_{i}\right\} S_{i}\{I\} \quad \text { for } i=1, \ldots, n \\
\{I\} \text { do } \square_{i=1}^{n} B_{i} \neg S_{i} \text { od }\left\{I \wedge \bigwedge_{i=1}^{n} \forall \vec{o}_{i}: \vec{T}_{i} \neg B_{i}\right\} \\
\text { where } I \in \mathcal{F}(\emptyset, \Pi) .
\end{gathered}
$$

As this proof rule is relatively complete [Apt84], it can prove (a). That is, there exists an eligibility-aware global assertion $I \in \mathcal{F}(\emptyset, \Pi)$ that supports a derivation from the premises of (DO-OD) to the correctness formula (a). In this derivation, we have $B_{i} \equiv g_{i} \wedge \neg \pi_{i}$ and $S_{i} \equiv a_{i} ; \pi_{i}:=$ true. The existence of the derivation means that the following hold:

$$
\begin{gathered}
\left\{I \wedge g_{i} \wedge \neg \pi_{i}\right\} a_{i} ; \pi_{i}:=\operatorname{true}\{I\} \quad \text { for } i=1, \ldots, n \\
p \wedge \bigwedge_{i=1}^{n} \forall \vec{o}_{i}: \vec{T}_{i} \neg \pi_{i} \rightarrow I \\
I \wedge \bigwedge_{i=1}^{n} \forall \vec{o}_{i}: \vec{T}_{i}\left(\neg g_{i} \vee \pi_{i}\right) \rightarrow q .
\end{gathered}
$$

Let us note $I_{\Delta} \equiv I\left[\delta_{i} / \pi_{i}\right]_{i=1}^{n}$ the global assertion from $\mathcal{F}(\emptyset, \Delta)$ obtained by substituting in $I$ the eligibility ghost variables $\delta_{i}$ for the ghost variables $\pi_{i}$. 
We define the assertions $p_{i}$ and $q_{i}$ as follows:

$$
p_{i} \stackrel{\text { def }}{=} I_{\Delta} \quad \text { and } \quad q_{i} \stackrel{\text { def }}{=} I_{\Delta} \quad \text { for } i=1, \ldots, n .
$$

It follows from $I_{\Delta}$ having no free variable that the $p_{i}, q_{i}$ are focused on the rule $r_{i}$, and that $p_{i}(\vec{x}) \equiv I_{\Delta}$ and $q_{i}(\vec{x}) \equiv I_{\Delta}$ for any $\vec{x} \in \operatorname{Var}^{m_{i}}$.

- Validity of the individual correctness formulas. For any $i \in\{1, \ldots, n\}$, we show below that the eligibility-aware correctness formula $\left\{p_{i} \wedge \neg \delta_{i}\right\} r_{i}^{*}\left\{q_{i}\right\}$ holds.

Consider two states $s$ and $s^{\prime}$ and a valuation $\nu$, such that $s, \nu \models p_{i} \wedge \neg \delta_{i}$ and $s \stackrel{\left(r_{i}^{*}, \nu\right)}{\longrightarrow} s^{\prime}$. We examine in turn the cases where the eligibility-aware rule instance $\left(r_{i}^{*}, \nu\right)$ is applicable in $s$, or not.

On the one hand, if the rule instance is not applicable in $s$, then it is not selectable and the state $s^{\prime}$ is defined as identical to $s$. It follows then from $s, \nu \models p_{i}$ and from the fact that, by definition, $p_{i} \equiv q_{i}$, that we have $s^{\prime}, \nu \models q_{i}$, which proves the correctness formula $\left\{p_{i} \wedge \neg \delta_{i}\right\} r_{i}^{*}\left\{q_{i}\right\}$ in this case.

If, on the other hand, the rule instance is applicable in $s$, then $s, \nu \models g_{i}$. Given the definition of $p_{i}$, we have

$$
s, \nu \models I_{\Delta} \wedge g_{i} \wedge \neg \delta_{i} .
$$

This expresses in particular that the rule instance is selectable: the state $s^{\prime}$ is defined by $\left\langle s, s^{\prime}\right\rangle, \nu \models \hat{a}_{i} \wedge \hat{\delta}_{i}$, as stated in Section 9.1.3.

Let us define $s_{\Pi}$ and $s_{\Pi}^{\prime}$ as the states that interpret all attribute symbols and ghost variables as in $s$ and $s^{\prime}$, respectively, except that $s_{\Pi}$ and $s_{\Pi}^{\prime}$ interpret the ghost variables $\pi_{i}$ in the same way as $s$ and $s^{\prime}$ interpret the ghost variables $\delta_{i}$. With this definition of $s_{\Pi}$, and since the rule guard $g_{i}$ contains no ghost variable, we have from (e) that

$$
s_{\Pi}, \nu \models I \wedge g_{i} \wedge \neg \pi_{i} .
$$

Similarly, since the rule action $a_{i}$, and hence the related transition assertion $\hat{a}_{i}$, contain no ghost variable, we have from the transition assertion that defines $s^{\prime}$

$$
\left\langle s_{\Pi}, s_{\Pi}^{\prime}\right\rangle, \nu \models \hat{a}_{i} \wedge \hat{\pi}_{i} \text { (true). }
$$

The $i$-th correctness formula in (b) allows us to derive from (f) and (g) that

$$
s_{\Pi}^{\prime}, \nu \models I .
$$

It follows that $s^{\prime}, \nu \models I_{\Delta}$, that is, $s^{\prime}, \nu \models q_{i}$. This proves the correctness formula $\left\{p_{i} \wedge \neg \delta_{i}\right\} r_{i}^{*}\left\{q_{i}\right\}$.

- Freedom from cross-rule interference. For any $i, j \in\{1, \ldots, n\}$ with $i \neq j$, we show below that the correctness formula $\left\{p_{i} \wedge p_{j} \wedge \neg \delta_{i} \wedge \neg \delta_{j}\right\} r_{j}^{*}\left\{p_{i} \wedge \neg \delta_{i}\right\}$ holds. The proof of the companion correctness formula involving $q_{i} \wedge \delta_{i}$ is similar.

Consider two states $s$ and $s^{\prime}$ and a valuation $\nu$, such that

$$
s, \nu \models p_{i} \wedge p_{j} \wedge \neg \delta_{i} \wedge \neg \delta_{j}
$$

and $s \stackrel{\left(r_{j}^{*}, \nu\right)}{\longrightarrow} s^{\prime}$. We examine in turn the cases where the eligibility-aware rule instance $\left(r_{j}^{*}, \nu\right)$ is applicable in $s$, or not. 
On the one hand, if the rule instance is not applicable in $s$, then it is not selectable and the state $s^{\prime}$ is defined as identical to $s$. It follows then from (h) that $s^{\prime}, \nu \models p_{i} \wedge \neg \delta_{i}$, which proves the correctness formula in this case.

If, on the other hand, the rule instance is applicable in $s$, meaning that we have $s, \nu \models g_{j}$, then (h) and the definition of the assertions $p_{i}$ give

$$
s, \nu \models I_{\Delta} \wedge g_{j} \wedge \neg \delta_{i} \wedge \neg \delta_{j} .
$$

This expresses in particular that the rule instance $\left(r_{j}^{*}, \nu\right)$ is selectable: the state $s^{\prime}$ is defined by $\left\langle s, s^{\prime}\right\rangle, \nu \models \hat{a}_{j} \wedge \hat{\delta}_{j}$.

Let us define the states $s_{\Pi}$ and $s_{\Pi}^{\prime}$ in the same way as for the proof of the individual correctness formulas. The equation (i) and the definition of $s^{\prime}$ give

$$
\begin{aligned}
s_{\Pi}, \nu & \models I \wedge g_{j} \wedge \neg \pi_{j} \\
\left\langle s_{\Pi}, s_{\Pi}^{\prime}\right\rangle, \nu & \models \hat{a}_{j} \wedge \hat{\pi}_{j} \text { (true). }
\end{aligned}
$$

The $j$-th correctness formula in (b) allows us to derive from the above that

$$
s_{\Pi}^{\prime}, \nu \models I .
$$

In addition, since $i \neq j$, the transition assertion $\left\langle s_{\Pi}, s_{\Pi}^{\prime}\right\rangle, \nu \models \hat{\pi}_{j}$ (true) gives in particular that $\left\langle s_{\Pi}, s_{\Pi}^{\prime}\right\rangle, \nu \models \pi_{i}^{\prime}=\pi_{i}$. And since $s_{\Pi}, \nu \models \neg \pi_{i}$, it follows that $s_{\Pi}^{\prime}, \nu \models \neg \pi_{i}$. This with (j) gives $s_{\Pi}^{\prime}, \nu \models I \wedge \neg \pi_{i}$, hence

$$
s^{\prime}, \nu \models I_{\Delta} \wedge \neg \delta_{i}
$$

that is, $s^{\prime}, \nu \models p_{i} \wedge \neg \delta_{i}$, which proves the desired correctness formula.

- Freedom from interference due to aliasing. For any $i \in\{1, \ldots, n\}$ and $\vec{x} \in$ $\mathrm{Var}^{m_{i}}$, we show below that the third interference freedom correctness formula $\left\{\vec{x} \neq \vec{o}_{i} \wedge p_{i}(\vec{x}) \wedge p_{i} \wedge \neg \delta_{i}(\vec{x}) \wedge \neg \delta_{i}\right\} r_{i}^{*}\left\{p_{i}(\vec{x}) \wedge \neg \delta_{i}(\vec{x})\right\}$ holds. The proof of the companion correctness formula involving $q_{i}(\vec{x}) \wedge \delta_{i}(\vec{x})$ is similar.

Consider two states $s$ and $s^{\prime}$ and a valuation $\nu$, such that

$$
s, \nu \models \vec{x} \neq \vec{o}_{i} \wedge p_{i}(\vec{x}) \wedge p_{i} \wedge \neg \delta_{i}(\vec{x}) \wedge \neg \delta_{i}
$$

and $s \stackrel{\left(r_{i}^{*}, \nu\right)}{\longrightarrow} s^{\prime}$. We examine in turn the cases where the eligibility-aware rule instance $\left(r_{i}^{*}, \nu\right)$ is applicable in $s$, or not.

On the one hand, if the rule instance is not applicable in $s$, then it is not selectable and the state $s^{\prime}$ is defined as identical to $s$. It follows then from $(\mathrm{k})$ that $s^{\prime}, \nu \models p_{i}(\vec{x}) \wedge \neg \delta_{i}(\vec{x})$, which proves the targeted correctness formula in this case.

If, on the other hand, the rule instance is applicable in $s$, meaning that we have $s, \nu \models g_{i}$, then (k) and the definition of $p_{i}$ give

$$
s, \nu \models I_{\Delta} \wedge g_{i} \wedge \neg \delta_{i}(\vec{x}) \wedge \neg \delta_{i} .
$$

This expresses in particular that the rule instance $\left(r_{i}^{*}, \nu\right)$ is selectable: the state $s^{\prime}$ is defined by $\left\langle s, s^{\prime}\right\rangle, \nu \models \hat{a}_{i} \wedge \hat{\delta}_{i}$.

Let us define the states $s_{\Pi}$ and $s_{\Pi}^{\prime}$ in the same way as for the proof of the individual correctness formulas. The equation (l) and the definition of $s^{\prime}$ give

$$
\begin{aligned}
s_{\Pi}, \nu & \models I \wedge g_{i} \wedge \neg \pi_{i} \\
\left\langle s_{\Pi}, s_{\Pi}^{\prime}\right\rangle, \nu & \models \hat{a}_{i} \wedge \hat{\pi}_{i} \text { (true) } .
\end{aligned}
$$


The $i$-th correctness formula in (b) allows us to derive from the above that

$$
s_{\Pi}^{\prime}, \nu \models I .
$$

In addition, since $\nu \models \vec{x} \neq \vec{o}_{i}$, the transition assertion $\left\langle s_{\Pi}, s_{\Pi}^{\prime}\right\rangle, \nu \models \hat{\pi}_{i}$ (true) gives in particular that $\left\langle s_{\Pi}, s_{\Pi}^{\prime}\right\rangle, \nu \models \pi_{i}^{\prime}(\vec{x})=\pi_{i}(\vec{x})$. And since from (l) we also have $s_{\Pi}, \nu \models \neg \pi_{i}(\vec{x})$, it follows that $s_{\Pi}^{\prime}, \nu \models \neg \pi_{i}(\vec{x})$. This with (m) gives $s_{\Pi}^{\prime}, \nu \models I \wedge \neg \pi_{i}(\vec{x})$, hence

$$
s^{\prime}, \nu \models I_{\Delta} \wedge \neg \delta_{i}(\vec{x})
$$

that is, $s^{\prime}, \nu \models p_{i}(\vec{x}) \wedge \neg \delta_{i}(\vec{x})$, which proves the targeted correctness formula.

- Derivation by (PR2). We have proven that the eligibility-aware correctness formulas $\left\{p_{i} \wedge \neg \delta_{i}\right\} r_{i}^{*}\left\{q_{i}\right\}$ focused on the individual rules hold and are interference free. We can therefore derive the following correctness formula for the rule program $\mathcal{R}$ by proof rule (PR2):

$$
\left\{\bigwedge_{i=1}^{n} \forall \vec{o}_{i}: \vec{T}_{i}\left(p_{i} \wedge \neg \delta_{i}\right)\right\} \mathcal{R}\left\{\bigwedge_{i=1}^{n} \forall \vec{o}_{i}: \vec{T}_{i}\left(q_{i} \wedge\left(\neg g_{i} \vee \delta_{i}\right)\right)\right\} .
$$

We need to prove that the correctness formula $\{p\} \mathcal{R}\{q\}$ can be derived from this correctness formula.

For this purpose, consider two states $s$ and $s^{\prime}$, such that $s \models p$ and $s \stackrel{\mathcal{R}}{\longrightarrow} s^{\prime}$. Part (i) of Lemma 9.1 indicates that since $s$ is the initial state of a rule program execution we have $s \models \bigwedge_{i=1}^{n} \forall \vec{o}_{i}: \vec{T}_{i} \neg \delta_{i}$. Therefore, we have

$$
s \models p \wedge \bigwedge_{i=1}^{n} \forall \vec{o}_{i}: \vec{T}_{i} \neg \delta_{i}
$$

The implication (c) states that $p \wedge \bigwedge_{i=1}^{n} \forall \vec{o}_{i}: \vec{T}_{i} \neg \pi_{i} \rightarrow I$. Let us substitute the ghost variables $\delta_{i}$ for the ghost variables $\pi_{i}$ in this implication. Since $p \in$ $\mathcal{F}(\emptyset, \emptyset)$ contains no ghost variable, the substitution gives

$$
p \wedge \bigwedge_{i=1}^{n} \forall \vec{o}_{i}: \vec{T}_{i} \neg \delta_{i} \rightarrow I_{\Delta}
$$

This with (o) gives that $s \models I_{\Delta}$, and furthermore $s \models I_{\Delta} \wedge \bigwedge_{i=1}^{n} \forall \vec{o}_{i}: \vec{T}_{i} \neg \delta_{i}$, which is the precondition of the correctness formula $(\mathrm{n})$ given the definition of $p_{i}$. As $s \stackrel{\mathcal{R}}{\longrightarrow} s^{\prime}$, the state $s^{\prime}$ satisfies the postcondition of the correctness formula $(\mathrm{n})$, that is, given the definition of $q_{i}$ :

$$
s^{\prime} \models I_{\Delta} \wedge \bigwedge_{i=1}^{n} \forall \vec{o}_{i}: \vec{T}_{i}\left(\neg g_{i} \vee \delta_{i}\right) .
$$

The implication (d) states that $I \wedge \bigwedge_{i=1}^{n} \forall \vec{o}_{i}: \vec{T}_{i}\left(\neg g_{i} \vee \pi_{i}\right) \rightarrow q$. By the same substitution as above, we obtain $I_{\Delta} \wedge \bigwedge_{i=1}^{n} \forall \vec{o}_{i}: \vec{T}_{i}\left(\neg g_{i} \vee \delta_{i}\right) \rightarrow q$. This with (p) gives that $s^{\prime} \models q$, proving the correctness formula

$$
\{p\} \mathcal{R}\{q\}
$$


- Summary. Given a rule program $\mathcal{R}=\left\{r_{1}, \ldots, r_{n}\right\}$ and a valid correctness formula $\{p\} \mathcal{R}\{q\}$ for this rule program, we have shown that there exists a collection of $2 n$ eligibility-aware assertions $p_{i}, q_{i} \in \mathcal{F}_{\Delta}\left(r_{i}\right)$ focused on the rules in the program, such that the correctness formulas $\left\{p_{i}\right\} r_{i}^{*}\left\{q_{i}\right\}$ hold, are interference free, and the correctness formula derived from them by (PR2) leads to $\{p\} \mathcal{R}\{q\}$. That is, we have given a proof of $\{p\} \mathcal{R}\{q\}$ by proof rule (PR2).

\subsection{Application of the Verification Method}

The application of our verification method has been discussed and illustrated in Section 8.4 in the simplified setting of Chapter 8. The present section reviews this subject in the light of the additional features of the general proof rule, namely eligibility and interference due to aliasing.

This section also provides a detailed illustration of an application of our verification method to an example. This example focuses on interference due to aliasing. The example in Section 8.4 addressed cross-rule interference; the ones in Section 10.1 further illustrate how to take eligibility into account.

\subsubsection{Approach}

To prove a correctness formula $\{p\} \mathcal{R}\{q\}$ on a rule program $\mathcal{R}$ with proof rule (PR2), a bottom-up approach similar to the one described in Section 8.4 has to be used.

That is, noting $\mathcal{R}=\left\{r_{1}, \ldots, r_{n}\right\}$, one should look for assertions $p_{i}, q_{i}$ with $i=1, \ldots, n$, which satisfy the following criteria:

(i) The assertions $p_{i}, q_{i}$ are focused on the corresponding rule, and are eligibility-aware; that is, $p_{i}, q_{i} \in \mathcal{F}_{\Delta}\left(r_{i}\right)$ for $i=1, \ldots, n$.

(ii) The eligibility-aware correctness formulas built with these assertions hold for each rule; that is, $\left\{p_{i} \wedge \neg \delta_{i}\right\} r_{i}^{*}\left\{q_{i}\right\}$ for $i=1, \ldots, n$.

(iii) These correctness formulas are free from cross-rule interference, that is, for $i, j=1, \ldots, n$ with $i \neq j$ :

$$
\begin{gathered}
\left\{p_{i} \wedge p_{j} \wedge \neg \delta_{i} \wedge \neg \delta_{j}\right\} r_{j}^{*}\left\{p_{i} \wedge \neg \delta_{i}\right\} \\
\left\{q_{i} \wedge p_{j} \wedge \delta_{i} \wedge \neg \delta_{j}\right\} r_{j}^{*}\left\{q_{i} \wedge \delta_{i}\right\} .
\end{gathered}
$$

(iv) They are also free from interference due to aliasing, that is in addition to the above, for $i=1, \ldots, n$ and $\vec{x} \in \operatorname{Var}^{m_{i}}$ :

$$
\begin{gathered}
\left\{\vec{x} \neq \vec{o}_{i} \wedge p_{i}(\vec{x}) \wedge p_{i} \wedge \neg \delta_{i}(\vec{x}) \wedge \neg \delta_{i}\right\} r_{i}^{*}\left\{p_{i}(\vec{x}) \wedge \neg \delta_{i}(\vec{x})\right\} \\
\left\{\vec{x} \neq \vec{o}_{i} \wedge q_{i}(\vec{x}) \wedge p_{i} \wedge \delta_{i}(\vec{x}) \wedge \neg \delta_{i}\right\} r_{i}^{*}\left\{q_{i}(\vec{x}) \wedge \delta_{i}(\vec{x})\right\} .
\end{gathered}
$$

(v) The correctness formula derived by the proof rule leads to the target one, that is,

$$
\begin{gathered}
p \rightarrow \bigwedge_{i=1}^{n} \forall \vec{o}_{i}: \vec{T}_{i}\left(p_{i} \wedge \neg \delta_{i}\right) \\
\bigwedge_{i=1}^{n} \forall \vec{o}_{i}: \vec{T}_{i}\left(q_{i} \wedge\left(\neg g_{i} \vee \delta_{i}\right)\right) \rightarrow q .
\end{gathered}
$$


In contrast with (PR1), proof rule (PR2) takes eligibility and aliasing into account. This has an effect on the assertions $p_{i}, q_{i}$ and on the correctness formulas formed with them.

To account for eligibility, correctness formulas are now eligibility-aware, with pre- and postconditions containing eligibility ghost variables. The occurrences of these ghost variables come both from the assertions $p_{i}, q_{i}$, and explicitly from the correctness formulas themselves, as in the precondition of the individual correctness formulas $\left\{p_{i} \wedge \neg \delta_{i}\right\} r_{i}^{*}\left\{q_{i}\right\}$.

Note that in many examples, the simpler though stronger correctness formula $\left\{p_{i}\right\} r_{i}^{*}\left\{q_{i}\right\}$ holds as well. The conjunction with $\neg \delta_{i}$ in the precondition is required in particular when the assertion $p_{i}$ implies the guard $g_{i}$, but does not imply the postcondition $q_{i}$, as is the case in the example discussed in Section 10.1.2. The conjunction with $\neg \delta_{i}$ then ensures that the executions of $r_{i}$ considered when verifying the correctness formula effectively applies the rule action.

To address aliasing, the assertions $p_{i}, q_{i}$ should include not only the eligibility ghost variables for the other rules, but also the one for the rule they are focused on.

Another consequence of aliasing is that the number of applicable instances of a given rule for a given updated object is not bounded by a constant, but instead depends on the number of objects in working memory. Consider the following two rules, extracted from Section 8.1:

$$
\begin{gathered}
r_{1}(p: \text { Person }): \text { p.age }>60 \rightarrow \text { p.bonus }:=\text { p.bonus }+10 \\
r_{4}(c: \text { Card, } p: \text { Person }): \text { p.bonus } \geq 20 \rightarrow \text { c.points }:=\text { c.points }+10
\end{gathered}
$$

An execution of the rule program $\left\{r_{1}, r_{4}\right\}$ contains at most one applicable instance of the rule $r_{1}$ on a given instance of Person. On the other hand, such an execution contains as many applicable instances of $r_{4}$ on a given instance of Card, as there are instances of Person with a bonus greater than or equal to 20 .

This consequence of aliasing usually materializes as weaker postconditions in correctness formulas, compared to attributes that are updated in unary rules. More expressivity could be achieved by extending the language of assertions with constructs expressing the number of objects in working memory that satisfy a given condition.

Finally, the complexity of the conclusion of (PR2) is both a tool and a burden. On the one hand, the presence of eligibility ghost variables helps selecting those disjuncts in the assertions $p_{i}, q_{i}$ that are relevant in the first and last configurations of an execution of the rule program. On the other hand, the preand postconditions of a global correctness formula to prove include no eligibility ghost variables. This makes step (v) in the approach described above more difficult to perform. However Lemma 9.6 below is of help in this task.

Lemma 9.6. Let $\mathcal{R}=\left\{r_{1}, \ldots, r_{n}\right\}$ be a rule program and $\Delta$ the set of its eligibility ghost variables; let $p, q \in \mathcal{F}(\emptyset, \emptyset)$ be two global assertions containing no eligibility ghost variable. One has

$$
\{p\} \mathcal{R}\{q\} \quad \text { iff } \quad\left\{p \wedge \bigwedge_{i=1}^{n} \forall \vec{o}_{i}: \vec{T}_{i} \neg \delta_{i}\right\} \mathcal{R}\left\{q \wedge \bigwedge_{i=1}^{n} \forall \vec{o}_{i}: \vec{T}_{i}\left(\neg g_{i} \vee \delta_{i}\right)\right\} .
$$

Proof. We examine each direction of the equivalence in turn. 
Proof of the $\Rightarrow$ direction. Consider two states $s$ and $s^{\prime}$, such that $s \models p \wedge$ $\bigwedge_{i=1}^{n} \forall \vec{o}_{i}: \vec{T}_{i} \neg \delta_{i}$ and $s \stackrel{\mathcal{R}}{\rightarrow} s^{\prime}$. Assume that $\{p\} \mathcal{R}\{q\}$ holds.

We have in particular $s \models p$ and hence by the assumed correctness formula we have $s^{\prime} \models q$.

In addition, since $s^{\prime}$ is the final state in an execution of $\mathcal{R}$, we have by Lemma 9.1 that $s^{\prime} \models \bigwedge_{i=1}^{n} \forall \vec{o}_{i}: \vec{T}_{i}\left(\neg g_{i} \vee \delta_{i}\right)$.

Therefore, we have $s^{\prime} \models q \wedge \bigwedge_{i=1}^{n} \forall \vec{o}_{i}: \vec{T}_{i}\left(\neg g_{i} \vee \delta_{i}\right)$, which proves the targeted correctness formula and this direction of the equivalence.

Proof of the $\Leftarrow$ direction. Consider two states $s$ and $s^{\prime}$, such that $s \models p$ and $s \stackrel{\mathcal{R}}{\longrightarrow}$ $s^{\prime}$. Assume that $\left\{p \wedge \bigwedge_{i=1}^{n} \forall \vec{o}_{i}: \vec{T}_{i} \neg \delta_{i}\right\} \mathcal{R}\left\{q \wedge \bigwedge_{i=1}^{n} \forall \vec{o}_{i}: \vec{T}_{i}\left(\neg g_{i} \vee \delta_{i}\right)\right\}$ holds.

Since $s$ is the initial state in an execution of $\mathcal{R}$, we have by Lemma 9.1 that $s \models \bigwedge_{i=1}^{n} \forall \vec{o}_{i}: \vec{T}_{i} \neg \delta_{i}$. Together with $s \models p$ this gives by the assumed correctness formula that $s^{\prime} \models q \wedge \bigwedge_{i=1}^{n} \forall \vec{o}_{i}: \vec{T}_{i}\left(\neg g_{i} \vee \delta_{i}\right)$.

That is in particular, $s^{\prime} \models q$, which proves the targeted correctness formula and this direction of the equivalence.

The pre- and postconditions in the conclusion of (PR2) are not exactly in the form of the right-hand side correctness formula in Lemma 9.6. Yet, if two global assertions $p^{\natural}, q^{\natural} \in \mathcal{F}(\emptyset, \emptyset)$ containing no eligibility ghost variable can be found, such that

$$
\begin{aligned}
& \bigwedge_{i=1}^{n} \forall \vec{o}_{i}: \vec{T}_{i}\left(p_{i} \wedge \neg \delta_{i}\right) \quad \Leftrightarrow \quad p^{\natural} \wedge \bigwedge_{i=1}^{n} \forall \vec{o}_{i}: \vec{T}_{i} \neg \delta_{i} \\
& \bigwedge_{i=1}^{n} \forall \vec{o}_{i}: \vec{T}_{i}\left(q_{i} \wedge\left(\neg g_{i} \vee \delta_{i}\right)\right) \quad \Leftrightarrow \quad q^{\natural} \wedge \bigwedge_{i=1}^{n} \forall \vec{o}_{i}: \vec{T}_{i}\left(\neg g_{i} \vee \delta_{i}\right)
\end{aligned}
$$

then step (v) can be performed by proving

$$
p \rightarrow p^{\natural} \quad \text { and } \quad q^{\natural} \rightarrow q .
$$

The observation of the pre- and postconditions of conclusion of (PR2) might suggest to split the inner conjunctions in these assertions, to find candidates for $p^{\natural}$ and $q^{\natural}$. However, $\bigwedge_{i=1}^{n} \forall \vec{o}_{i}: \vec{T}_{i} p_{i}$ is usually not eligible for $p^{\natural}$, as it contains eligibility ghost variables. Similarly, $\bigwedge_{i=1}^{n} \forall \vec{o}_{i}: \vec{T}_{i} q_{i}$ usually contains eligibility ghost variables and hence is not eligible for $q^{\natural}$.

\subsubsection{Example}

Consider a rule program responsible for accumulating discount points on a collective discount card, based on the characteristics of the owners of the card. The rule program $\mathcal{R}=\left\{r_{1}, r_{2}\right\}$ below is an example of such a program.

$$
\begin{aligned}
& r_{1}(\mathrm{u}: \text { Card, } \mathrm{x}: \text { Person }): \mathrm{x} \cdot \text { age }>60 \rightarrow \mathrm{u} \cdot \text { discount }:=\mathrm{u} \cdot \mathrm{discount}+5 \\
& \mathrm{r}_{2}(\mathrm{v}: \text { Card, } \mathrm{y}: \text { Person }): \mathrm{y} \cdot \mathrm{cat}=\text { Gold } \rightarrow \mathrm{v} \cdot \mathrm{discount}:=\mathrm{v} \cdot \text { discount }+10
\end{aligned}
$$

Imagine that we want to certify that, at the end of any execution that starts with empty discount cards, the number of discount points on any card is a 
non-negative multiple of 5 . This amounts to proving the following correctness formula:

$$
\{\forall c \text { : Card }(\text { c.discount }=0)\} \mathcal{R}\{\forall c \text { : Card } \exists k(k \geq 0 \wedge c \text {.discount }=5 k)\} \text {. }
$$

In the rest of this example, we note $\mathrm{P}$ and $\mathrm{C}$ for the type names Person and Card, and $d$ for the discount attribute symbol. The correctness formula to prove is thus

$$
\{\forall c: \mathrm{C}(c . d=0)\} \mathcal{R}\{\forall c: \mathrm{C} \exists k(k \geq 0 \wedge c . d=5 k)\} .
$$

We propose the following eligibility-aware assertions as the pre- and postconditions of the correctness formulas focused on the individual rules:

$$
\begin{aligned}
& p_{1} \equiv u . d=0 \vee \exists k(k \geq 0 \wedge u . d=5 k) \wedge \exists p: \mathrm{P}\left(\delta_{1}(u, p) \vee \delta_{2}(u, p)\right) \\
& p_{2} \equiv v . d=0 \vee \exists k(k \geq 0 \wedge v . d=5 k) \wedge \exists p: \mathrm{P}\left(\delta_{1}(v, p) \vee \delta_{2}(v, p)\right) \\
& q_{1} \equiv \exists k(k \geq 0 \wedge u . d=5 k) \\
& q_{2} \equiv \exists k(k \geq 0 \wedge v . d=5 k) .
\end{aligned}
$$

These assertions express the evolution of the discount points of a given card, noted $u$ in $r_{1}$ and in the assertions focused on this rule, and $v$ for rule $r_{2}$. When no instance of any rule on the given discount card has been applied, it has zero points. For a card $u$, this situation corresponds to $\forall p: \mathrm{P}\left(\neg \delta_{1}(u, p) \wedge \neg \delta_{2}(u, p)\right)$. As soon as one instance or $r_{1}$ or $r_{2}$ on the given discount card has been applied, that is, when $\exists p: \mathrm{P}\left(\delta_{1}(u, p) \vee \delta_{2}(u, p)\right)$, all we know is that the discount points have increased by 5 or 10 a certain number of times.

In this respect, the assertion $p_{1}$ could be equivalently written as

$$
\begin{aligned}
u . d=0 \wedge \forall p: \mathrm{P}\left(\neg \delta_{1}(u, p)\right. & \left.\wedge \neg \delta_{2}(u, p)\right) \\
\vee & \exists k(k \geq 1 \wedge u . d=5 k) \wedge \exists p: \mathrm{P}\left(\delta_{1}(u, p) \vee \delta_{2}(u, p)\right)
\end{aligned}
$$

and similarly for $p_{2}$. However, $p_{1}$ is implied by the above formula, and suffices to apply our verification method.

Let us now put (PR2) to work on these assertions to prove (9.2).

\section{The individual correctness formulas hold}

We verify below that the eligibility-aware correctness formula $\left\{p_{1} \wedge \neg \delta_{1}\right\} r_{1}^{*}\left\{q_{1}\right\}$ holds. A similar reasoning gives $\left\{p_{2} \wedge \neg \delta_{2}\right\} r_{2}^{*}\left\{q_{2}\right\}$.

Consider two states $s$ and $s^{\prime}$ and a valuation $\nu$, such that $s, \nu \models p_{1} \wedge \neg \delta_{1}$ and $s \stackrel{\left(r_{1}^{*}, \nu\right)}{\longrightarrow} s^{\prime}$. For the correctness formula to hold, we must have $s^{\prime}, \nu \models q_{1}$. We distinguish the cases where the eligibility-aware rule instance $\left(r_{1}^{*}, \nu\right)$ is applicable in $s$, and hence selectable, or not.

- If the rule instance is selectable, the state $s^{\prime}$ is defined by $\left\langle s, s^{\prime}\right\rangle, \nu \models \hat{a}_{1} \wedge \hat{\delta}_{1}$, which gives in particular (see remark (i) on page 130)

$$
\left\langle s, s^{\prime}\right\rangle, \nu \models u . d^{\prime}=u . d+5 .
$$

By hypothesis we have $s, \nu \models p_{1}$. Given the definition of $p_{1}$ we have either (or both) of

$$
s, \nu \models u . d=0
$$


or

$$
s, \nu \models \exists k(k \geq 0 \wedge u . d=5 k) \wedge \exists p: \mathrm{P}\left(\delta_{1}(u, p) \vee \delta_{2}(u, p)\right)
$$

that is in all cases,

$$
s, \nu \models u . d=5 k \quad \text { for some } k \geq 0 \text {. }
$$

Applying the transition described by (9.3) to the above gives $s^{\prime}, \nu \models u . d=$ $5 k+5$, that is,

$$
s^{\prime}, \nu \models \exists k(k \geq 1 \wedge u . d=5 k)
$$

which implies $s^{\prime}, \nu \models q_{1}$.

- If the rule instance is not selectable, the state $s^{\prime}$ is defined as identical to $s$. As above, $s, \nu \models p_{1}$ gives in all cases

$$
s^{\prime}, \nu \models \exists k(k \geq 0 \wedge u . d=5 k)
$$

which is $s^{\prime}, \nu \models q_{1}$.

- Summary. We have seen that, whether the eligibility-aware rule instance $\left(r_{i}^{*}, \nu\right)$ is selectable or not, we have $s^{\prime}, \nu \models q_{1}$. The eligibility-aware correctness formula $\left\{p_{1} \wedge \neg \delta_{1}\right\} r_{1}^{*}\left\{q_{1}\right\}$ therefore holds.

\section{Interference freedom between rules: preconditions}

We start by verifying the preservation of $p_{1} \wedge \neg \delta_{1}$ by the eligibility-aware execution of $r_{2}$, that is, that $\left\{p_{1} \wedge p_{2} \wedge \neg \delta_{1} \wedge \neg \delta_{2}\right\} r_{2}^{*}\left\{p_{1} \wedge \neg \delta_{1}\right\}$ holds. A similar reasoning gives the preservation of $p_{2} \wedge \neg \delta_{2}$ by the eligibility-aware execution of $r_{1}$.

Consider two states $s$ and $s^{\prime}$ and a valuation $\nu$, such that

$$
s, \nu \models p_{1} \wedge p_{2} \wedge \neg \delta_{1} \wedge \neg \delta_{2}
$$

and $s \stackrel{\left(r_{2}^{*}, \nu\right)}{\longrightarrow} s^{\prime}$. For the correctness formula to hold, we must have $s^{\prime}, \nu \models$ $p_{1} \wedge \neg \delta_{1}$. We distinguish the following cases.

- If the rule instance $\left(r_{2}^{*}, \nu\right)$ is not selectable in $s$, the state $s^{\prime}$ is defined as identical to $s$. It follows directly from (9.4) that $s^{\prime}, \nu \models p_{1} \wedge \neg \delta_{1}$.

- If the rule instance $\left(r_{2}^{*}, \nu\right)$ is selectable in $s$ and $\nu \models u \neq v$ : Since the rule instance is selectable, the state $s^{\prime}$ is defined by $\left\langle s, s^{\prime}\right\rangle, \nu \models \hat{a}_{2} \wedge \hat{\delta}_{2}$, which since $\nu \models u \neq v$ gives in particular (see remark (ii) on page 130)

$$
\left\langle s, s^{\prime}\right\rangle, \nu \models u . d^{\prime}=u . d \wedge \forall p: \mathrm{P}\left(\delta_{1}^{\prime}(u, p)=\delta_{1}(u, p) \wedge \delta_{2}^{\prime}(u, p)=\delta_{2}(u, p)\right) .
$$

It follows from (9.4) that $s, \nu \models p_{1} \wedge \neg \delta_{1}$, that is,

$s, \nu \models\left(u . d=0 \vee \exists k(k \geq 0 \wedge u . d=5 k) \wedge \exists p: \mathrm{P}\left(\delta_{1}(u, p) \vee \delta_{2}(u, p)\right)\right) \wedge \neg \delta_{1}(u, x)$.

As the transition described by (9.5) leaves $u . d$ unchanged, as well as $\delta_{1}(u, \cdot)$ and $\delta_{2}(u, \cdot)$, we have $s^{\prime}, \nu \models p_{1} \wedge \neg \delta_{1}$. 
- If the rule instance $\left(r_{2}^{*}, \nu\right)$ is selectable in $s$ and $\nu \models u=v$ : Since the rule instance is selectable, the state $s^{\prime}$ is defined by $\left\langle s, s^{\prime}\right\rangle, \nu \models \hat{a}_{2} \wedge \hat{\delta}_{2}$, which since $\nu \models u=v$ gives in particular (see remarks (i) and (ii) on page 130)

$$
\left\langle s, s^{\prime}\right\rangle, \nu \models u . d^{\prime}=u . d+10 \wedge \delta_{1}^{\prime}(u, x)=\delta_{1}(u, x) \wedge \delta_{2}^{\prime}(u, x) .
$$

By (9.4) we have $s, \nu \models p_{1}$, which implies $s, \nu \models u . d=5 k$ for some $k \geq 0$. By the first conjunct in (9.6) this gives $s^{\prime}, \nu \models u . d=5 k+10$. Together with the last conjunct in (9.6) this means that

$$
s^{\prime}, \nu \models \exists k(k \geq 2 \wedge u . d=5 k) \wedge \exists p: \mathrm{P}\left(p=y \wedge \delta_{2}(u, p)\right)
$$

which implies $s^{\prime}, \nu \models p_{1}$.

From (9.4) we have $s, \nu \models \neg \delta_{1}$, which is a shorthand for $s, \nu \models \neg \delta_{1}(u, x)$. Hence, by the second conjunct in (9.6), we have $s^{\prime}, \nu \models \neg \delta_{1}$. We finally have $s^{\prime}, \nu \models p_{1} \wedge \neg \delta_{1}$.

- Summary. We have seen that, whether the eligibility-aware rule instance $\left(r_{2}^{*}, \nu\right)$ is selectable or not, and whether aliasing occurs or not, we have $s^{\prime}, \nu \models$ $p_{1} \wedge \neg \delta_{1}$. The correctness formula $\left\{p_{1} \wedge p_{2} \wedge \neg \delta_{1} \wedge \neg \delta_{2}\right\} r_{2}^{*}\left\{p_{1} \wedge \neg \delta_{1}\right\}$ therefore holds.

\section{Interference freedom between rules: postconditions}

We now verify the preservation of $q_{1} \wedge \delta_{1}$ by the eligibility-aware execution of $r_{2}$, that is, that $\left\{q_{1} \wedge p_{2} \wedge \delta_{1} \wedge \neg \delta_{2}\right\} r_{2}^{*}\left\{q_{1} \wedge \delta_{1}\right\}$ holds. A similar reasoning gives the preservation of $q_{2} \wedge \delta_{2}$ by the eligibility-aware execution of $r_{1}$.

Consider two states $s$ and $s^{\prime}$ and a valuation $\nu$, such that

$$
s, \nu \models q_{1} \wedge p_{2} \wedge \delta_{1} \wedge \neg \delta_{2}
$$

and $s \stackrel{\left(r_{2}^{*}, \nu\right)}{\longrightarrow} s^{\prime}$. For the correctness formula to hold, we must have $s^{\prime}, \nu \models q_{1} \wedge \delta_{1}$. We distinguish the same cases as for the preconditions. However, we do not give the detail of the first two cases, as their descriptions are almost identical to the corresponding cases for the preconditions.

When the rule instance $\left(r_{2}^{*}, \nu\right)$ is selectable in $s$ and $\nu \models u=v$, the definition of $s^{\prime}$ by $\left\langle s, s^{\prime}\right\rangle, \nu \models \hat{a}_{2} \wedge \hat{\delta}_{2}$ gives in particular

$$
\left\langle s, s^{\prime}\right\rangle, \nu \models u . d^{\prime}=u . d+10 \wedge \delta_{1}^{\prime}(u, x)=\delta_{1}(u, x) \wedge \delta_{2}^{\prime}(u, x) .
$$

It follows from (9.7) that $s, \nu \models q_{1} \wedge \delta_{1}$, that is,

$$
s, \nu \models \exists k(k \geq 0 \wedge u . d=5 k) \wedge \delta_{1}(u, x) .
$$

The transition described by (9.8) increases $u . d$ by 10 , and leaves $\delta_{1}(u, x)$ unchanged. We thus have

$$
s^{\prime}, \nu \models \exists k(k \geq 2 \wedge u . d=5 k) \wedge \delta_{1}(u, x)
$$

which implies $s^{\prime}, \nu \models q_{1} \wedge \delta_{1}$.

The correctness formula $\left\{q_{1} \wedge p_{2} \wedge \delta_{1} \wedge \neg \delta_{2}\right\} r_{2}^{*}\left\{q_{1} \wedge \delta_{1}\right\}$ therefore holds. 


\section{Interference freedom between instances of a rule}

We consider now two variables $t$ and $z$, and we verify the preservation of $p_{1}(t, z) \wedge$ $\neg \delta_{1}(t, z)$ by the eligibility-aware execution of $r_{1}$ when $(t, z) \neq(u, x)$. That is, we verify $\left\{(t, z) \neq(u, x) \wedge p_{1}(t, z) \wedge p_{1} \wedge \neg \delta_{1}(t, z) \wedge \neg \delta_{1}\right\} r_{1}^{*}\left\{p_{1}(t, z) \wedge \neg \delta_{1}(t, z)\right\}$.

A similar reasoning gives the preservation of $p_{2}(t, z) \wedge \neg \delta_{2}(t, z)$ by the eligibility-aware execution of $r_{2}$ when $(t, z) \neq(v, y)$. Also, the preservation of $q_{1}(t, z) \wedge \delta_{1}(t, z)$ by the eligibility-aware execution of $r_{1}$ when $(t, z) \neq(u, x)$, and the preservation of $q_{2}(t, z) \wedge \delta_{2}(t, z)$ by the eligibility-aware execution of $r_{2}$ when $(t, z) \neq(v, y)$, are verified in the same way.

Consider two states $s$ and $s^{\prime}$ and a valuation $\nu$, such that

$$
s, \nu \models(t, z) \neq(u, x) \wedge p_{1}(t, z) \wedge p_{1} \wedge \neg \delta_{1}(t, z) \wedge \neg \delta_{1}
$$

and $s \stackrel{\left(r_{1}^{*}, \nu\right)}{\longrightarrow} s^{\prime}$. For the correctness formula to hold, we must have $s^{\prime}, \nu \models$ $p_{1}(t, z) \wedge \neg \delta_{1}(t, z)$. We distinguish the same cases as before.

- If the rule instance $\left(r_{1}^{*}, \nu\right)$ is not selectable in $s$, the state $s^{\prime}$ is defined as identical to $s$. It follows directly from (9.9) that $s^{\prime}, \nu \models p_{1}(t, z) \wedge \neg \delta_{1}(t, z)$.

- If the rule instance $\left(r_{1}^{*}, \nu\right)$ is selectable in $s$ and $\nu \models t \neq u$, the definition of $s^{\prime}$ by $\left\langle s, s^{\prime}\right\rangle, \nu \models \hat{a}_{1} \wedge \hat{\delta}_{1}$ gives in particular

$$
\left\langle s, s^{\prime}\right\rangle, \nu \models t . d^{\prime}=t . d \wedge \forall p: \mathrm{P}\left(\delta_{1}^{\prime}(t, p)=\delta_{1}(t, p) \wedge \delta_{2}^{\prime}(t, p)=\delta_{2}(t, p)\right) .
$$

It follows from (9.9) that $s, \nu \models p_{1}(t, z) \wedge \neg \delta_{1}(t, z)$, that is,

$$
s, \nu \models\left(t . d=0 \vee \exists k(k \geq 0 \wedge t . d=5 k) \wedge \exists p: \mathrm{P}\left(\delta_{1}(t, p) \vee \delta_{2}(t, p)\right)\right) \wedge \neg \delta_{1}(t, z) .
$$

As the transition described by (9.10) leaves $t . d$ unchanged, as well as $\delta_{1}(t, \cdot)$ and $\delta_{2}(t, \cdot)$, we have $s^{\prime}, \nu \models p_{1}(t, z) \wedge \neg \delta_{1}(t, z)$.

- If the rule instance $\left(r_{1}^{*}, \nu\right)$ is selectable in $s$ and $\nu \models t=u$, the definition of $s^{\prime}$ by $\left\langle s, s^{\prime}\right\rangle, \nu \models \hat{a}_{1} \wedge \hat{\delta}_{1}$ gives in particular

$$
\left\langle s, s^{\prime}\right\rangle, \nu \models t . d^{\prime}=t . d+5 \wedge \delta_{1}^{\prime}(t, x) \wedge \forall p: \mathrm{P}\left(p \neq x \rightarrow \delta_{1}^{\prime}(t, p)=\delta_{1}(t, p)\right) .
$$

By (9.9) we have $s, \nu \models p_{1}(t, z)$, which implies $s, \nu \models t . d=5 k$ for some $k \geq$ 0 . By the first conjunct in (9.11) this gives $s^{\prime}, \nu \models t . d=5 k+5$. Together with the second conjunct in (9.11) this means that

$$
s^{\prime}, \nu \models \exists k(k \geq 1 \wedge t . d=5 k) \wedge \exists p: \mathrm{P}\left(p=x \wedge \delta_{1}(t, p)\right)
$$

which implies $s^{\prime}, \nu \models p_{1}(t, z)$.

From (9.9) we also have $s, \nu \models \neg \delta_{1}(t, z)$. Since by hypothesis $\nu \models(t, z) \neq$ $(u, x)$ and $\nu \models t=u$, we must have $\nu \models z \neq x$. Hence, by the last conjunct in (9.11), we have $s^{\prime}, \nu \models \neg \delta_{1}(t, z)$. It follows that $s^{\prime}, \nu \models p_{1}(t, z) \wedge \neg \delta_{1}(t, z)$.

- Summary. We have seen that, whether the eligibility-aware rule instance $\left(r_{1}^{*}, \nu\right)$ is selectable or not, and whether aliasing occurs or not, we have $s^{\prime}, \nu \models$ $p_{1}(t, z) \wedge \neg \delta_{1}(t, z)$. The expected correctness formula therefore holds. 


\section{Proving the global correctness formula}

In the above, we have verified all the premises of proof rule (PR2): we can derive its conclusion. Let us note $\{\varphi\} \mathcal{R}\{\psi\}$ this derived correctness formula: we have

$$
\begin{aligned}
\varphi & \stackrel{\text { def }}{\equiv} \bigwedge_{i=1}^{2} \forall \vec{o}_{i}: \vec{T}_{i}\left(p_{i} \wedge \neg \delta_{i}\right) \\
& \equiv \forall u: \mathrm{C} \forall x: \mathrm{P}\left(p_{1} \wedge \neg \delta_{1}\right) \wedge \forall v: \mathrm{C} \forall y: \mathrm{P}\left(p_{2} \wedge \neg \delta_{2}\right) \\
\psi & \stackrel{\text { def }}{\equiv} \bigwedge_{i=1}^{2} \forall \vec{o}_{i}: \vec{T}_{i}\left(q_{i} \wedge\left(\neg g_{i} \vee \delta_{i}\right)\right) \\
& \equiv \forall u: \mathrm{C} \forall x: \mathrm{P}\left(q_{1} \wedge\left(\neg g_{1} \vee \delta_{1}\right)\right) \wedge \forall v: \mathrm{C} \forall y: \mathrm{P}\left(q_{2} \wedge\left(\neg g_{2} \vee \delta_{2}\right)\right) .
\end{aligned}
$$

Let us also note $\{p\} \mathcal{R}\{q\}$ the correctness formula (9.2) to prove, that is,

$$
\begin{aligned}
& p \stackrel{\text { def }}{\equiv} \forall c: \mathrm{C}(c . d=0) \\
& q \stackrel{\text { def }}{\equiv} \forall c: \mathrm{C} \exists k(k \geq 0 \wedge c . d=5 k)) .
\end{aligned}
$$

To complete the proof of (9.2) with (PR2), we need to show that $\{\varphi\} \mathcal{R}\{\psi\}$ leads to $\{p\} \mathcal{R}\{q\}$.

- Detail of $\varphi$. From their definitions, it can be seen that the only free variables in $p_{1}$ and $p_{2}$ are $u$ and $v$, respectively. We can therefore rename in $\varphi$ the card variables to $c$ and the person variables to $p$. We get

$$
\varphi \Leftrightarrow \forall c: \mathrm{C} \forall p: \mathrm{P}\left(p_{1}(c, p) \wedge p_{2}(c, p) \wedge \neg \delta_{1}(c, p) \wedge \neg \delta_{2}(c, p)\right) .
$$

However, we have

$p_{1}(c, p) \equiv p_{2}(c, p) \equiv c . d=0 \vee \exists k(k \geq 0 \wedge c . d=5 k) \wedge \exists p: \mathrm{P}\left(\delta_{1}(c, p) \vee \delta_{2}(c, p)\right)$.

Let us note $p_{0} \stackrel{\text { def }}{=} p_{1}(c, p) \equiv p_{2}(c, p)$ : we have

$$
\varphi \Leftrightarrow \forall c: \mathrm{C} \forall p: \mathrm{P}\left(p_{0} \wedge \neg \delta_{1}(c, p) \wedge \neg \delta_{2}(c, p)\right)
$$

and since $c$ is the only free variable in $p_{0}$,

$$
\varphi \Leftrightarrow \forall c: \mathrm{C}\left(p_{0} \wedge \forall p: \mathrm{P}\left(\neg \delta_{1}(c, p) \wedge \neg \delta_{2}(c, p)\right)\right) .
$$

That is, by expanding $p_{0}$,

$$
\begin{aligned}
\varphi \Leftrightarrow \forall c: \mathrm{C} & \left(\left(c . d=0 \vee \exists k(k \geq 0 \wedge c . d=5 k) \wedge \exists p: \mathrm{P}\left(\delta_{1}(c, p) \vee \delta_{2}(c, p)\right)\right)\right. \\
& \left.\wedge \forall p: \mathrm{P}\left(\neg \delta_{1}(c, p) \wedge \neg \delta_{2}(c, p)\right)\right)
\end{aligned}
$$

which, after simplification, gives

$$
\begin{aligned}
\varphi & \Leftrightarrow \forall c: \mathrm{C}(c . d=0) \wedge \forall c: \mathrm{C} \forall p: \mathrm{P}\left(\neg \delta_{1}(c, p) \wedge \neg \delta_{2}(c, p)\right) \\
& \Leftrightarrow \forall c: \mathrm{C}(c . d=0) \wedge \forall u: \mathrm{C} \forall x: \mathrm{P} \neg \delta_{1} \wedge \forall v: \mathrm{C} \forall y: \mathrm{P} \neg \delta_{2} .
\end{aligned}
$$


- Detail of $\psi$. Let us remind that we have

$$
\psi \equiv \forall u: \mathrm{C} \forall x: \mathrm{P}\left(q_{1} \wedge\left(\neg g_{1} \vee \delta_{1}\right)\right) \wedge \forall v: \mathrm{C} \forall y: \mathrm{P}\left(q_{2} \wedge\left(\neg g_{2} \vee \delta_{2}\right)\right)
$$

The free variables in $q_{1}$ and $q_{2}$ are $u$ and $v$, respectively. We can therefore rename them to $c$ and the person variables to $p$ :

$$
\begin{aligned}
\psi & \Leftrightarrow \forall c: \mathrm{C} \forall p: \mathrm{P}\left(q_{1}(c, p) \wedge q_{2}(c, p)\right) \\
& \wedge \forall u: \mathrm{C} \forall x: \mathrm{P}\left(\neg g_{1} \vee \delta_{1}\right) \wedge \forall v: \mathrm{C} \forall y: \mathrm{P}\left(\neg g_{2} \vee \delta_{2}\right) .
\end{aligned}
$$

However, we have

$$
q_{1}(c, p) \equiv q_{2}(c, p) \equiv \exists k(k \geq 0 \wedge c . d=5 k)
$$

Let us note $q_{0} \stackrel{\text { def }}{\equiv} q_{1}(c, p) \equiv q_{2}(c, p)$. Since $c$ is the only free variable in $q_{0}$, we have

$$
\psi \Leftrightarrow \forall c: \mathrm{C} q_{0} \wedge \forall u: \mathrm{C} \forall x: \mathrm{P}\left(\neg g_{1} \vee \delta_{1}\right) \wedge \forall v: \mathrm{C} \forall y: \mathrm{P}\left(\neg g_{2} \vee \delta_{2}\right)
$$

- Conclusion. By proof rule (PR2), we derived $\{\varphi\} \mathcal{R}\{\psi\}$. The details of $\varphi$ and $\psi$ have given that

and

$$
\begin{aligned}
\varphi & \Leftrightarrow \forall c: \mathrm{C}(c . d=0) \wedge \forall u: \mathrm{C} \forall x: \mathrm{P} \neg \delta_{1} \wedge \forall v: \mathrm{C} \forall y: \mathrm{P} \neg \delta_{2} \\
& \Leftrightarrow p \wedge \bigwedge_{i=1}^{2} \forall \vec{o}_{i}: \vec{T}_{i} \neg \delta_{i}
\end{aligned}
$$

$$
\begin{aligned}
\psi & \Leftrightarrow \forall c: \mathrm{C} \exists k(k \geq 0 \wedge c . d=5 k) \\
& \wedge \forall u: \mathrm{C} \forall x: \mathrm{P}\left(\neg g_{1} \vee \delta_{1}\right) \wedge \forall v: \mathrm{C} \forall y: \mathrm{P}\left(\neg g_{2} \vee \delta_{2}\right) \\
& \Leftrightarrow q \wedge \bigwedge_{i=1}^{2} \forall \vec{o}_{i}: \vec{T}_{i}\left(\neg g_{i} \vee \delta_{i}\right) .
\end{aligned}
$$

The correctness formula $\{\varphi\} \mathcal{R}\{\psi\}$ is thus equivalent to

$$
\left\{p \wedge \bigwedge_{i=1}^{2} \forall \vec{o}_{i}: \vec{T}_{i} \neg \delta_{i}\right\} \mathcal{R}\left\{q \wedge \bigwedge_{i=1}^{2} \forall \vec{o}_{i}: \vec{T}_{i}\left(\neg g_{i} \vee \delta_{i}\right)\right\} .
$$

By Lemma 9.6, this correctness formula is equivalent to $\{p\} \mathcal{R}\{q\}$, which is therefore proven.

\section{Discussion}

Given the rule program $\mathcal{R}$ reminded below, we have proven with proof rule (PR2) that at the end of any execution that starts with empty discount cards, the number of discount points on any card is a non-negative multiple of 5 .

$$
\begin{aligned}
& \mathrm{r}_{1}(\mathrm{u}: \text { Card, } \mathrm{x}: \text { Person }): \mathrm{x} . \text { age }>60 \rightarrow \mathrm{u} \cdot \text { discount }:=\mathrm{u} \cdot \text { discount }+5 \\
& \mathrm{r}_{2}(\mathrm{v}: \text { Card, } \mathrm{y}: \text { Person }): \mathrm{y} \cdot \mathrm{cat}=\mathrm{Gold} \rightarrow \mathrm{v} \cdot \mathrm{discount}:=\mathrm{v} \cdot \mathrm{discount}+10
\end{aligned}
$$

Although the verification of the premises of the rules is long, it is mainly mechanical. The creative part in the application of proof rule (PR2) resides in finding the right $p_{i}, q_{i}$ assertions. 
The present example focuses on interference due to aliasing, as for a given instance of Card the condition c.discount $=0$ is subject to being invalidated by the application of any rule instance that includes this object. As discussed in Section 9.2.2, this kind of interference is addressed by both groups of interference freedom correctness formulas in the premises of the proof rule.

The examples in Section 10.1 prove correctness formulas for unary rule programs, which are immune to interference due to aliasing. These examples further illustrate how to take eligibility into account, independently of cross-rule interference. 


\section{Chapter 10}

\section{Specialized Proof Rules}

The proof rule exposed in the previous chapter handles rules with an arbitrary number of variables, it takes the eligibility of rule instances into account, and addresses both cross-rule interference and interference due to aliasing. Naturally, this proof rule can also be used to prove correctness formulas for rule programs when eligibility or either kind of interference plays no role. In such cases however, using a specialized proof rule is simpler, in that fewer premises have to be verified, or eligibility ghost variables can be omitted.

Chapter 8 introduced such a specialized proof rule, suited to correctness formulas for rule programs made of unary rules and where eligibility needs not be taken into account. The present chapter provides additional proof rules, specialized for some classes of rule programs and correctness formulas to prove on them.

\subsection{Eligibility-Aware Unary Rules}

The simplified setting of Chapter 8 includes programs made of unary rules, and correctness formulas for which eligibility needs not be taken into account. The previous chapter relieved both simplifications. However, the case of unary rule programs that take eligibility into account is of interest. The present section presents a sound and relatively complete proof rule for this case.

Unary rules are defined in Section 8.2, with a short reminder below. They have the simplifying property that they cannot cause, or suffer from, interference due to aliasing.

A unary rule has only one rule variable. In this context, we simplify the notations: given a rule program $\mathcal{R}=\left\{r_{1}, \ldots, r_{n}\right\}$ made of unary rules, we note the rules $r_{i}=\left(o_{i}, T_{i}, g_{i}, a_{i}\right)$ with $a_{i} \equiv o_{i} \cdot f_{r_{i}}:=e_{r_{i}}$, for $i=1, \ldots, n$. We use the notation $\forall \vec{o}: \vec{T}$ as an abbreviation for $\forall o_{1}: T_{1} \ldots \forall o_{n}: T_{n}$.

In Chapter 8, we exposed the foundations of our verification method with rule programs made of unary rules, in cases where eligibility need not be taken into account. This restriction eliminates the need for eligibility ghost variables. Section 8.4 gives an example of such a case.

Here, we address the case of correctness formulas for unary rule programs 
in which eligibility must be taken into account. Eligibility ghost variables must therefore be included in the correctness formulas focused on the rules. Still there is no possibility of interference due to aliasing, and the last group of premises in proof rule (PR2) can be omitted. This property is formalized in the lemma below.

Lemma 10.1. Let $\mathcal{R}=\left\{r_{1}, \ldots, r_{n}\right\}$ be a rule program made of unary rules and $\Delta$ the set of its eligibility ghost variables. Given an $i \in\{1, \ldots, n\}$, two eligibility-aware assertions $p_{i}, q_{i} \in \mathcal{F}_{\Delta}\left(r_{i}\right)$ focused on the rule $r_{i}$, and a variable $x$, the following correctness formulas hold:

$$
\begin{gathered}
\left\{x \neq o_{i} \wedge p_{i}(x) \wedge p_{i} \wedge \neg \delta_{i}(x) \wedge \neg \delta_{i}\right\} r_{i}^{*}\left\{p_{i}(x) \wedge \neg \delta_{i}(x)\right\} \\
\left\{x \neq o_{i} \wedge q_{i}(x) \wedge p_{i} \wedge \delta_{i}(x) \wedge \neg \delta_{i}\right\} r_{i}^{*}\left\{q_{i}(x) \wedge \delta_{i}(x)\right\}
\end{gathered}
$$

Proof. Consider two states $s$ and $s^{\prime}$ and a valuation $\nu$, such that $s \stackrel{\left(r_{i}^{*}, \nu\right)}{\longrightarrow} s^{\prime}$.

If the rule instance $\left(r_{i}^{*}, \nu\right)$ is not selectable in $s$, then the state $s^{\prime}$ is defined as identical to $s$. As a consequence, if $s$ satisfies the precondition of either correctness formula, then $s^{\prime}$ satisfies its conclusion.

If the rule instance $\left(r_{i}^{*}, \nu\right)$ is selectable in $s$, then the state $s^{\prime}$ is defined by $\left\langle s, s^{\prime}\right\rangle, \nu \models \hat{a}_{i} \wedge \hat{\delta}_{i}$, as stated in Section 9.1.3. If $\nu \models x \neq o_{i}$ then this transition gives in particular

$$
\left\langle s, s^{\prime}\right\rangle, \nu \models \bigwedge_{f \in \text { Attr }} x \cdot f^{\prime}=x . f \wedge \bigwedge_{\delta \in \Delta} \delta^{\prime}(x)=\delta(x) .
$$

Since the rules, and hence the assertions $p_{i}, q_{i}$ and the eligibility ghost variables, are unary, it follows that the truth values of $p_{i}(x)$, of $q_{i}(x)$, and of $\delta_{i}(x)$ are the same in $s$ and in $s^{\prime}$. As a consequence, if $s$ satisfies the precondition of either correctness formula, then $s^{\prime}$ satisfies its conclusion.

For both correctness formulas, we have proven that any eligibility-aware execution of $r_{i}$ from a state that satisfies the precondition of the correctness formula results in a state that satisfies its postcondition. This proves the validity of the correctness formulas, and hence the lemma.

\subsubsection{Proof Rule}

Theorem 10.2. Let $\mathcal{R}=\left\{r_{1}, \ldots, r_{n}\right\}$ be a rule program made of unary rules and $\Delta$ its set of eligibility ghost variables. For $i=1, \ldots, n$ let $p_{i}, q_{i} \in$ $\mathcal{F}_{\Delta}\left(r_{i}\right)$ be assertions focused on the rule $r_{i}$, possibly containing eligibility ghost variables. The proof rule below is sound and relatively complete (in the sense of Section 9.4).

$$
\begin{gathered}
\left\{p_{i} \wedge \neg \delta_{i}\right\} r_{i}^{*}\left\{q_{i}\right\} \quad \text { for } i=1, \ldots, n \\
\left\{p_{i} \wedge p_{j} \wedge \neg \delta_{i} \wedge \neg \delta_{j}\right\} r_{j}^{*}\left\{p_{i} \wedge \neg \delta_{i}\right\} \\
\left.\left\{q_{i} \wedge p_{j} \wedge \delta_{i} \wedge \neg \delta_{j}\right\} r_{j}^{*}\left\{q_{i} \wedge \delta_{i}\right\}\right\} \text { for } i, j=1, \ldots, n \text { with } i \neq j \\
\left\{\bigwedge_{i=1}^{n} \forall o_{i}: T_{i}\left(p_{i} \wedge \neg \delta_{i}\right)\right\} \mathcal{R}\left\{\bigwedge_{i=1}^{n} \forall o_{i}: T_{i}\left(q_{i} \wedge\left(\neg g_{i} \vee \delta_{i}\right)\right)\right\}
\end{gathered}
$$

Proof. The soundness of (PR3) is a consequence of Theorem 9.2 stating the soundness of (PR2), and of Lemma 10.1 above. 
Its relative completeness for unary rule programs is deduced below from the relative completeness of (PR2) stated by Theorem 9.4. The relative completeness of (PR2) implies that for any valid global correctness formula $\{p\} \mathcal{R}\{q\}$, there exist eligibility-aware assertions $p_{i}, q_{i}$ for $i=1, \ldots, n$, such that:

(i) The assertions are focused on the corresponding rules, that is, $p_{i}, q_{i} \in$ $\mathcal{F}_{\Delta}\left(r_{i}\right)$.

(ii) The eligibility-aware correctness formulas $\left\{p_{i} \wedge \neg \delta_{i}\right\} r_{i}^{*}\left\{q_{i}\right\}$ hold.

(iii) They are interference free, with in particular

$$
\left.\begin{array}{c}
\left\{p_{i} \wedge p_{j} \wedge \neg \delta_{i} \wedge \neg \delta_{j}\right\} r_{j}^{*}\left\{p_{i} \wedge \neg \delta_{i}\right\} \\
\left\{q_{i} \wedge p_{j} \wedge \delta_{i} \wedge \neg \delta_{j}\right\} r_{j}^{*}\left\{q_{i} \wedge \delta_{i}\right\}
\end{array}\right\} \text { for } i, j=1, \ldots, n \text { with } i \neq j .
$$

(iv) The correctness formula derived from them by (PR2), which is the same as the one in the conclusion of (PR3), leads to $\{p\} \mathcal{R}\{q\}$.

The first three conditions correspond to the premises of (PR3). Together with the last one, they express the relative completeness of (PR3) for unary rule programs.

As noted at the end of Section 8.3, it is often the case in programs made of unary rules that the sole variables of the rules in the program all have the same type $T$. In this case, the conclusion of the proof rule can be reorganized, and the proof rule can be written

$$
\begin{aligned}
& \left\{p_{i} \wedge \neg \delta_{i}\right\} r_{i}^{*}\left\{q_{i}\right\} \quad \text { for } i=1, \ldots, n \\
& \left.\begin{array}{c}
\left\{p_{i} \wedge p_{j} \wedge \neg \delta_{i} \wedge \neg \delta_{j}\right\} r_{j}^{*}\left\{p_{i} \wedge \neg \delta_{i}\right\} \\
\left\{q_{i} \wedge p_{j} \wedge \delta_{i} \wedge \neg \delta_{j}\right\} r_{j}^{*}\left\{q_{i} \wedge \delta_{i}\right\}
\end{array}\right\} \text { for } i, j=1, \ldots, n \text { with } i \neq j \\
& \frac{\left.\left\{q_{i} \wedge p_{j} \wedge \delta_{i} \wedge \neg \delta_{j}\right\} r_{j}^{*}\left\{q_{i} \wedge \delta_{i}\right\}\right\} \text { for } i, j=1, \ldots, n \text { with } i \neq j}{\left\{\forall o: T \bigwedge_{i=1}^{n}\left(p_{i}(o) \wedge \neg \delta_{i}(o)\right)\right\} \mathcal{R}\left\{\forall o: T \bigwedge_{i=1}^{n}\left(q_{i}(o) \wedge\left(\neg g_{i}(o) \vee \delta_{i}(o)\right)\right)\right\}}
\end{aligned}
$$

Example. As a first illustration of the interest of (PR3), let us return to the rule program and correctness formula that served as a counterexample in the proof of the incompleteness of (PR1) in Section 8.5.

Consider the rule program $\mathcal{R}=\left\{r_{1}, r_{2}\right\}$ and the correctness formula below:

$$
\begin{aligned}
& \mathrm{r}_{1}(\mathrm{x}: \mathrm{T}): \mathrm{x} \cdot \mathrm{a} \geq 20 \rightarrow \mathrm{x} \cdot \mathrm{b}:=\mathrm{x} \cdot \mathrm{a}-10 \\
& \mathrm{r}_{2}(\mathrm{y}: \mathrm{T}): \mathrm{y} \cdot \mathrm{b}>70 \rightarrow \mathrm{y} \cdot \mathrm{b}:=70 \\
& \{\forall o: T(o . b=0)\} \mathcal{R}\{\forall o: T(o . b \leq 70)\} .
\end{aligned}
$$

We propose the following assertions as pre- and postconditions of the correctness formulas focused on each rule:

$$
\begin{array}{ll}
p_{1} \equiv x \cdot b=0 \wedge \neg \delta_{2}(x) & q_{1} \equiv \text { true } \\
p_{2} \equiv \text { true } & q_{2} \equiv y \cdot b \leq 70 .
\end{array}
$$

- The individual correctness formulas hold. Because $q_{1} \equiv$ true, the correctness formula $\left\{p_{1} \wedge \neg \delta_{1}\right\} r_{1}^{*}\left\{q_{1}\right\}$ holds trivially. On the other hand, $\left\{p_{2} \wedge \neg \delta_{2}\right\} r_{2}^{*}\left\{q_{2}\right\}$ is obvious. 
- Interference freedom. Let us start with $\left\{p_{1} \wedge p_{2} \wedge \neg \delta_{1} \wedge \neg \delta_{2}\right\} r_{2}^{*}\left\{p_{1} \wedge \neg \delta_{1}\right\}$, and consider two states $s$ and $s^{\prime}$ and a valuation $\nu$, such that $s, \nu \models p_{1} \wedge$ $p_{2} \wedge \neg \delta_{1} \wedge \neg \delta_{2}$ and $s \stackrel{\left(r_{2}^{*}, \nu\right)}{\longrightarrow} s^{\prime}$. If the rule instance $\left(r_{2}^{*}, \nu\right)$ is not applicable, then $s^{\prime}=s$ and the correctness formula holds. If, on the other hand, $\left(r_{2}^{*}, \nu\right)$ is applicable, we have $s, \nu \models g_{2}$. Together with the hypothesis $s, \nu \models p_{1}$ this gives $s, \nu \models x . b=0 \wedge y . b>70$, which implies that $\nu \models x \neq y$. As a consequence, $p_{1}$ and $\delta_{1}$ are left unchanged by the execution of the rule instance, since their only free variable is $x$. Hence $s^{\prime}, \nu \models p_{1} \wedge \neg \delta_{1}$.

The correctness formula $\left\{p_{1} \wedge p_{2} \wedge \neg \delta_{1} \wedge \neg \delta_{2}\right\} r_{1}^{*}\left\{p_{2} \wedge \neg \delta_{2}\right\}$ has the same precondition: the same reasoning also leads to $x \neq y$ when the rule instance is applicable. Its execution therefore leaves $p_{2}$ and $\delta_{2}$ unchanged, and the postcondition holds.

The correctness formula expressing the preservation of $q_{1} \wedge \delta_{1}$ by $r_{2}^{*}$ boils down to $\left\{\delta_{1} \wedge \neg \delta_{2}\right\} r_{2}^{*}\left\{\delta_{1}\right\}$. Its precondition forces $x \neq y$, which implies that the execution of the rule $r_{2}$ leaves $\delta_{1}$ unchanged, and that the postcondition holds.

Finally, the correctness formula expressing the preservation of $q_{2} \wedge \delta_{2}$ by $r_{1}^{*}$ is $\left\{q_{2} \wedge p_{1} \wedge \delta_{2} \wedge \neg \delta_{1}\right\} r_{1}^{*}\left\{q_{2} \wedge \delta_{2}\right\}$. Its precondition is $y . b \leq 70 \wedge x . b=0 \wedge$ $\neg \delta_{2}(x) \wedge \delta_{2} \wedge \neg \delta_{1}$, in which $\neg \delta_{2}(x) \wedge \delta_{2}$ forces $x \neq y$. This again implies that the execution of the rule $r_{1}$ leaves $q_{2}$ and $\delta_{2}$ unchanged, and that the postcondition holds.

- Conclusion. As the premises of (PR3) hold, we can derive the following correctness formula by this proof rule:

$$
\begin{aligned}
\{\forall o: T(o . b=70 \wedge & \left.\left.\neg \delta_{2}(o) \wedge \neg \delta_{1}(o)\right)\right\} \mathcal{R} \\
& \left\{\forall o: T\left(\left(\neg g_{1}(o) \vee \delta_{1}(o)\right) \wedge o . b \leq 70 \wedge\left(\neg g_{2}(o) \vee \delta_{2}(o)\right)\right)\right\}
\end{aligned}
$$

Lemma 9.6 was proven for rules of arbitrary arity and hence holds for unary rules as well. By this lemma the correctness formula above is equivalent to

$$
\{\forall o: T(o . b=70)\} \mathcal{R}\{\forall o: T(o . b \leq 70)\}
$$

which was the correctness formula to prove.

Proof rule (PR3) is thus capable of proving any valid correctness formula on unary rule programs, even when eligibility has to be taken into account. Compared to the general proof rule (PR2) exposed in the previous chapter, it exploits the fact that rules are unary, and hence are free from interference due to aliasing.

\subsubsection{Example}

Imagine a rule program responsible for computing a bonus for customers out of contributions based on their ages and categories. Such a rule program could contain the two rules below, previously given as an example of unary rules in Section 8.2:

$$
\begin{aligned}
& r_{1}(x: P): x \cdot \text { age }>60 \rightarrow x \cdot \text { bonus }:=x \cdot \text { bonus }+10 \\
& r_{2}(y: P): y \cdot c a t=\text { Gold } \rightarrow \text { y.bonus }:=y \cdot \text { bonus }+15
\end{aligned}
$$


These two rules respectively increment the customer's bonus by 10 and 15 when the customer is senior or gold. Our concern here is to prove that the rule program $\mathcal{R}=\left\{r_{1}, r_{2}\right\}$ is confluent on senior gold customers. Assuming that the initial discount is limited to 30 , this concern could be represented by the following correctness formula for $\mathcal{R}$ :

$$
\{\forall o: \mathrm{P}(\text { o.age }>60 \wedge \text { o.bonus } \leq 30 \wedge \text { o.cat }=\mathrm{Gold})\} \mathcal{R}\{\forall o: \mathrm{P}(\text { o.bonus } \leq 55)\} .
$$

This correctness formula cannot be proven using (PR1), because the rules do not invalidate their guards. Therefore only the eligibility mechanism can prevent them from being applied several times. As a result and since their actions are not idempotent, trying to find pre- and postconditions for correctness formulas focused on them as done in Section 8.4 does not converge. That is, repeatedly weakening these assertions as to reflect the effect of the rules does not reach a fix point. Eligibility ghost variables help solve this problem.

\section{The approach}

As when applying the proof rules (PR1) and (PR2), the proof of a correctness formula $\{p\} \mathcal{R}\{q\}$ on a rule program $\mathcal{R}=\left\{r_{1}, \ldots, r_{n}\right\}$ using (PR3) is achieved by finding assertions $p_{i}, q_{i}$ focused on the rules in the program, such that the correctness formulas $\left\{p_{i} \wedge \neg \delta_{i}\right\} r_{i}^{*}\left\{q_{i}\right\}$ hold, are interference free, and the correctness formula derived from them by the proof rule leads to $\{p\} \mathcal{R}\{q\}$.

Here, the premises of the proof rule contain no correctness formulas specific to interference due to aliasing. In addition, since the rules in the program are unary, the assertions $p_{i}, q_{i}$ are unary as well. The verification of the crossrule interference freedom correctness formulas can therefore be simplified by Lemma 10.3 given below, which can be seen as the eligibility-aware version of Lemma 8.4.

Finally, Lemma 9.6 can be used to conclude $\{p\} \mathcal{R}\{q\}$ out of the correctness formula derived from the proof rule.

Lemma 10.3. Let $\mathcal{R}=\left\{r_{1}, \ldots, r_{n}\right\}$ be a rule program made of unary rules and $\Delta$ the set of its eligibility ghost variables; let $r_{i}=\left(o_{i}, T_{i}, g_{i}, a_{i}\right)$ be a rule from $\mathcal{R}$ and $\varphi \in \mathcal{F}(\{o\}, \Delta)$ an eligibility-aware unary assertion in the fresh variable $o$. If one has

$$
\left\{\varphi \wedge p_{i} \wedge g_{i} \wedge \neg \delta_{i} \wedge o_{i}=o\right\} r_{i}^{*}\{\varphi\}
$$

then one has

$$
\left\{\varphi \wedge p_{i}\right\} r_{i}^{*}\{\varphi\}
$$

Proof. Consider two states $s$ and $s^{\prime}$ and a valuation $\nu$, such that $s, \nu \models \varphi \wedge p_{i}$ and $s \stackrel{\left(r_{i}^{*}, \nu\right)}{\longrightarrow} s^{\prime}$. To prove the lemma, we must show that $s^{\prime}, \nu \models \varphi$.

- If $s, \nu \models \neg g_{i}$ or $s, \nu \models \delta_{i}$ then by definition of eligibility-aware rule instance execution (see Section 9.1.3), $s \stackrel{\left(r_{i}^{*}, \nu\right)}{\longrightarrow} s^{\prime}$ means that $s^{\prime}=s$. One then has $s^{\prime}, \nu \models \varphi$.

— If $s, \nu \models g_{i} \wedge \neg \delta_{i}$ and $\nu \models o_{i}=o$ then one has $s^{\prime}, \nu \models \varphi$ by hypothesis.

— If $s, \nu \models g_{i} \wedge \neg \delta_{i}$ and $\nu \models o_{i} \neq o$ then $s \stackrel{\left(r_{i}^{*}, \nu\right)}{\longrightarrow} s^{\prime}$ means that $\left\langle s, s^{\prime}\right\rangle, \nu \models$ $\hat{a}_{i} \wedge \hat{\delta}_{i}$, with in particular (see Section 9.1.3 again) $\left\langle s, s^{\prime}\right\rangle, \nu \models \bigwedge_{f \in \operatorname{Attr}}\left(o . f^{\prime}=\right.$ o.f $) \wedge \bigwedge_{\delta \in \Delta}\left(\delta^{\prime}(o)=\delta(o)\right)$. Since free $(\varphi)=\{o\}$ one then has $s^{\prime}, \nu \models \varphi$. 


\section{The assertions}

The rule program under consideration is $\mathcal{R}=\left\{r_{1}, r_{2}\right\}$ made of the two rules below:

$$
\begin{aligned}
& r_{1}(x: P): x \cdot \text { age }>60 \rightarrow x \cdot \text { bonus }:=x \cdot \text { bonus }+10 \\
& r_{2}(y: P): y \cdot c a t=\text { Gold } \rightarrow \text { y.bonus }:=y \cdot \text { bonus }+15
\end{aligned}
$$

For the rest of this section, we abbreviate the age, bonus and category attribute symbols to $a, b$, and $c$, respectively. The global correctness formula that we want to prove with proof rule (PR3) therefore reads:

$$
\{\forall o: \mathrm{P}(o . a>60 \wedge o . b \leq 30 \wedge o . c=\text { Gold })\} \mathcal{R}\{\forall o: \mathrm{P}(o . b \leq 55)\} .
$$

We propose the following eligibility-aware assertions as the pre- and postconditions of the correctness formulas focused on the individual rules:

$$
\begin{aligned}
& p_{1} \equiv x . a>60 \wedge\left(x . b \leq 30 \wedge \neg \delta_{2}(x) \vee x . b \leq 45 \wedge \delta_{2}(x)\right) \\
& q_{1} \equiv x . a>60 \wedge\left(x . b \leq 40 \wedge \neg \delta_{2}(x) \vee x . b \leq 55 \wedge \delta_{2}(x)\right) \\
& p_{2} \equiv y . c=\text { Gold } \wedge\left(y . b \leq 30 \wedge \neg \delta_{1}(y) \vee y . b \leq 40 \wedge \delta_{1}(y)\right) \\
& q_{2} \equiv y . c=\text { Gold } \wedge\left(y . b \leq 45 \wedge \neg \delta_{1}(y) \vee y . b \leq 55 \wedge \delta_{1}(y)\right) .
\end{aligned}
$$

In each of these assertions, the eligibility ghost variables are clearly used to take into account whether the other rule has been executed on the same object or not. In $p_{1}$ for example, if the rule $r_{2}$ has not yet been executed on the object held by $x$, then $\delta_{2}(x)$ will be false and the assertion will retain $x . b \leq 30$. If, on the other hand, $r_{2}$ has already been executed on the object held by $x$, then $\delta_{2}(x)$ will be true and the assertion will retain $x . b \leq 45$, that is, the initial 30 plus the increment brought in by $r_{2}$.

Below we expose the application of (PR3) in detail.

\section{The individual correctness formulas hold}

We start by verifying that the correctness formula $\left\{p_{1} \wedge \neg \delta_{1}\right\} r_{1}^{*}\left\{q_{1}\right\}$ holds. A similar reasoning gives that $\left\{p_{2} \wedge \neg \delta_{2}\right\} r_{2}^{*}\left\{q_{2}\right\}$ holds.

Consider two states $s$ and $s^{\prime}$ and a valuation $\nu$, such that $s, \nu \models p_{1} \wedge \neg \delta_{1}$ and $s \stackrel{\left(r_{1}^{*}, \nu\right)}{\longrightarrow} s^{\prime}$, that is,

$$
\begin{aligned}
s, \nu & \models x . a>60 \wedge\left(x . b \leq 30 \wedge \neg \delta_{2}(x) \vee x . b \leq 45 \wedge \delta_{2}(x)\right) \wedge \neg \delta_{1} \\
\left\langle s, s^{\prime}\right\rangle, \nu & \models x . b^{\prime}=x . b+10 \wedge \forall o\left(o \neq x \rightarrow o . b^{\prime}=o . b\right) \\
& \wedge \forall o\left(o . a^{\prime}=o . a \wedge o . c^{\prime}=o . c\right) \\
& \wedge \delta_{1}^{\prime}(x) \wedge \forall o\left(o \neq x \rightarrow \delta_{1}^{\prime}(o)=\delta_{1}(o)\right) \\
& \wedge \forall o\left(\delta_{2}^{\prime}(o)=\delta_{2}(o)\right) .
\end{aligned}
$$

That is in particular,

$$
\left\langle s, s^{\prime}\right\rangle, \nu \models x . a^{\prime}=x . a \wedge x \cdot b^{\prime}=x . b+10 \wedge \delta_{2}^{\prime}(x)=\delta_{2}(x) .
$$

It follows that

$$
s^{\prime}, \nu \models x . a>60 \wedge\left(x . b \leq 40 \wedge \neg \delta_{2}(x) \vee x . b \leq 55 \wedge \delta_{2}(x)\right)
$$


that is, $s^{\prime}, \nu \models q_{1}$, which establishes the targeted correctness formula.

Note that here, the correctness formula $\left\{p_{1} \wedge \neg \delta_{1}\right\} r_{1}^{*}\left\{q_{1}\right\}$ holds, as just proven, but the stronger $\left\{p_{1}\right\} r_{1}^{*}\left\{q_{1}\right\}$ does not. Indeed, consider a state $s$ and a valuation $\nu$, such that $s, \nu \models p_{1} \wedge \delta_{1}$. In this state, the eligibility-aware rule instance $\left(r_{1}^{*}, \nu\right)$ is not selectable, and hence $s \stackrel{\left(r_{1}^{*}, \nu\right)}{\longrightarrow} s$. For $\left\{p_{1}\right\} r_{1}^{*}\left\{q_{1}\right\}$ to hold, one should therefore have $p_{1} \rightarrow q_{1}$, which is not the case.

\section{Interference freedom}

We start by verifying that $\left\{p_{1} \wedge p_{2} \wedge \neg \delta_{1} \wedge \neg \delta_{2}\right\} r_{2}^{*}\left\{p_{1} \wedge \neg \delta_{1}\right\}$ holds. For this purpose, we consider two states $s$ and $s^{\prime}$ and a valuation $\nu$, such that $s \stackrel{\left(r_{2}^{*}, \nu\right)}{\longrightarrow} s^{\prime}$. By Lemma 10.3 it suffices to consider the case where $s, \nu \models p_{1}(y) \wedge p_{2} \wedge g_{2} \wedge$ $\neg \delta_{1}(y) \wedge \neg \delta_{2}$, and to check that $s^{\prime}, \nu \models p_{1}(y) \wedge \neg \delta_{1}(y)$.

In the precondition $p_{1}(y) \wedge p_{2} \wedge g_{2} \wedge \neg \delta_{1}(y) \wedge \neg \delta_{2}$ the disjunction in $p_{1}(y)$ simplifies with $\neg \delta_{2}$ to give $y . a>60 \wedge y . b \leq 30 \wedge \neg \delta_{2}$. Similarly $p_{2}$ simplifies with $\neg \delta_{1}(y)$. From these simplifications, we have

$$
s, \nu \models y . a>60 \wedge y . b \leq 30 \wedge y . c=\operatorname{Gold} \wedge \neg \delta_{1}(y) \wedge \neg \delta_{2}(y) .
$$

This makes the rule instance $\left(r_{2}^{*}, \nu\right)$ selectable. Since from $\left\langle s, s^{\prime}\right\rangle, \nu \models \hat{a}_{2} \wedge \hat{\delta}_{2}$ we have in particular

$$
\left\langle s, s^{\prime}\right\rangle, \nu \models y \cdot a^{\prime}=y \cdot a \wedge y \cdot b^{\prime}=y \cdot b+15 \wedge \delta_{1}^{\prime}(y)=\delta_{1}(y) \wedge \delta_{2}^{\prime}(y)
$$

it follows that

$$
s^{\prime}, \nu \models y . a>60 \wedge y . b \leq 45 \wedge \neg \delta_{1}(y) \wedge \delta_{2}(y)
$$

hence

$$
s^{\prime}, \nu \models y . a>60 \wedge\left(y . b \leq 30 \wedge \neg \delta_{2}(y) \vee y . b \leq 45 \wedge \delta_{2}(y)\right) \wedge \neg \delta_{1}(y)
$$

that is,

$$
s^{\prime}, \nu \models p_{1}(y) \wedge \neg \delta_{1}(y) .
$$

The verification of the other three correctness formulas expressing interference freedom follows the same reasoning: use Lemma 10.3 to restrict the precondition, and apply the effect of the rule action (that is, increment the bonus and swap the corresponding eligibility rule variable) to obtain the postcondition.

\section{Conclusion}

Having verified all the premises of (PR3), we can derive its conclusion, that is,

$$
\begin{aligned}
&\left\{\forall o: \mathrm{P}\left(p_{1}(o) \wedge\right.\right.\left.\left.p_{2}(o) \wedge \neg \delta_{1}(o) \wedge \neg \delta_{2}(o)\right)\right\} \mathcal{R} \\
&\left\{\forall o: \mathrm{P}\left(q_{1}(o) \wedge q_{2}(o) \wedge\left(\neg g_{1}(o) \vee \delta_{1}(o)\right) \wedge\left(\neg g_{2}(o) \vee \delta_{2}(o)\right)\right)\right\} .
\end{aligned}
$$

As with the correctness formulas expressing interference freedom, the assertions $p_{i}, q_{i}$ simplify in the pre- and postconditions of this correctness formula 
with the eligibility ghost variables. Namely, we have

$$
\begin{aligned}
p_{1}(o) \wedge \neg \delta_{2}(o) & \equiv o . a>60 \wedge o . b \leq 30 \wedge \neg \delta_{2}(o) \\
p_{2}(o) \wedge \neg \delta_{1}(o) & \equiv o . c=\text { Gold } \wedge o . b \leq 30 \wedge \neg \delta_{1}(o) \\
q_{1}(o) \wedge\left(\neg g_{2}(o) \vee \delta_{2}(o)\right) & \equiv o . a>60 \wedge o . b \leq 55 \wedge \delta_{2}(o) \vee q_{1}(o) \wedge o . c \neq \text { Gold } \\
q_{2}(o) \wedge\left(\neg g_{1}(o) \vee \delta_{1}(o)\right) & \equiv o . c=\text { Gold } \wedge o . b \leq 55 \wedge \delta_{1}(o) \vee q_{2}(o) \wedge o . a \leq 60 .
\end{aligned}
$$

The precondition of the correctness formula derived by (PR3) can therefore be written as

$$
\forall o: \mathrm{P}(o . a>60 \wedge o . b \leq 30 \wedge o . c=\text { Gold }) \wedge \forall o: \mathrm{P}\left(\neg \delta_{1}(o) \wedge \neg \delta_{2}(o)\right)
$$

while the postcondition, after eliminating the false disjuncts, can be written as

$$
\forall o: \mathrm{P}(o . a>60 \wedge o . b \leq 55 \wedge o . c=\mathrm{Gold}) \wedge \forall o: \mathrm{P}\left(\delta_{1}(o) \wedge \delta_{2}(o)\right) .
$$

By Lemma 9.6 this leads to the following correctness formula, which was the one to prove:

$$
\{\forall o: \mathrm{P}(o . a>60 \wedge o . b \leq 30 \wedge o . c=\text { Gold })\} \mathcal{R}\{\forall o: \mathrm{P}(o . b \leq 55)\} .
$$

\section{Discussion}

In the example above, the negated guards in the postcondition of the correctness formula derived by (PR3) are no longer present after simplification. This comes from the fact that each $p_{i}$ implied the corresponding guard $g_{i}$, which was left untouched by the rule actions.

In complementary cases, the negated guards would be visible in the postcondition of the final correctness formula. For example, in the following correctness formula where $o . a>60$ is no longer part of the precondition:

$$
\begin{aligned}
\{\forall o: \mathrm{P}(o . b \leq 30 \wedge o . c=\mathrm{Gold})\} \mathcal{R} \\
\{\forall o: \mathrm{P}((o . a \leq 60 \rightarrow o . b \leq 45) \wedge(o . a>60 \rightarrow o . b \leq 55))\} .
\end{aligned}
$$

\subsection{Aliasing-Free N-ary Rule Programs}

The interest of programs made of unary rules come from the fact that no aliasing can occur during their executions. There is another way to avoid aliasing: impose that the working memory must not contain more than one instance of each type. The present section reviews this approach and provides a sound and relatively complete proof rule for this case.

Unary rules, introduced in Chapter 8 and also discussed in Section 10.1 above, are an option to avoid interference due to aliasing. However this option imposes a strong constraint on the syntax of the rules in the program.

There is an alternative to this constraint, that can be used with rules of arbitrary arity, which we call $n$-ary rules. In this alternative option, the working memory must contain at most one instance of each type. This constraint on the contents of the working memory also ensures that no interference due to aliasing occurs. We formalize it in the following lemma. 
Lemma 10.4. Let $\mathcal{R}=\left\{r_{1}, \ldots, r_{n}\right\}$ be a rule program and $\Delta$ the set of its eligibility ghost variables. Consider an $i \in\{1, \ldots, n\}$, two eligibility-aware assertions $p_{i}, q_{i} \in \mathcal{F}_{\Delta}\left(r_{i}\right)$ focused on the rule $r_{i}$, and a tuple of variables $\vec{x} \in$ $\operatorname{Var}^{\left|r_{i}\right|}$. In executions of $\mathcal{R}$ on a working memory $\mathcal{M}$ with at most one instance per type, that is, such that

$$
\text { for each } T \in \text { Types }|\mathcal{M}(T)| \leq 1
$$

the following correctness formulas hold:

$$
\begin{gathered}
\left\{\vec{x} \neq \vec{o}_{i} \wedge p_{i}(\vec{x}) \wedge p_{i} \wedge \neg \delta_{i}(\vec{x}) \wedge \neg \delta_{i}\right\} r_{i}^{*}\left\{p_{i}(\vec{x}) \wedge \neg \delta_{i}(\vec{x})\right\} \\
\left\{\vec{x} \neq \vec{o}_{i} \wedge q_{i}(\vec{x}) \wedge p_{i} \wedge \delta_{i}(\vec{x}) \wedge \neg \delta_{i}\right\} r_{i}^{*}\left\{q_{i}(\vec{x}) \wedge \delta_{i}(\vec{x})\right\} .
\end{gathered}
$$

Proof. Both correctness formulas trivially hold because their preconditions are false, which we show below by contradiction.

Indeed, suppose that there exist a state $s$ and a valuation $\nu$, such that $s, \nu \models \vec{x} \neq \vec{o}_{i} \wedge p_{i}(\vec{x}) \wedge p_{i} \wedge \neg \delta_{i}(\vec{x}) \wedge \neg \delta_{i}$ or $s, \nu \models \vec{x} \neq \vec{o}_{i} \wedge q_{i}(\vec{x}) \wedge p_{i} \wedge \delta_{i}(\vec{x}) \wedge \neg \delta_{i}$. In both cases this means that $\nu \models \vec{x} \neq \vec{o}_{i}$.

Let us note $m_{i}=\left|r_{i}\right|$ the arity of the rule $r_{i}: \nu \models \vec{x} \neq \vec{o}_{i}$ means that there exists a $j \in\left\{1, \ldots, m_{i}\right\}$ such that $\nu\left(x_{j}\right) \neq \nu\left(o_{j}^{i}\right)$. However, since we are in an execution of a rule program, the valuation $\nu$ is well-typed for the type constraint $\left(\vec{o}_{i}: \vec{T}_{i}\right)$ induced by the rule, meaning that both $\nu\left(x_{j}\right) \in T_{j}^{i}$ and $\nu\left(o_{j}^{i}\right) \in T_{j}^{i}$. Hence a contradiction with $\left|\mathcal{M}\left(T_{j}^{i}\right)\right| \leq 1$.

In such a setting where rules can be of arbitrary arity but the working memory contains at most one instance of each type, the proof rule provided by the following theorem can be used.

Theorem 10.5. Let $\mathcal{R}=\left\{r_{1}, \ldots, r_{n}\right\}$ be a rule program and $\Delta$ its set of eligibility ghost variables. For $i=1, \ldots, n$ let $p_{i}, q_{i} \in \mathcal{F}_{\Delta}\left(r_{i}\right)$ be assertions focused on the rule $r_{i}$, possibly containing eligibility ghost variables. The proof rule below is sound and relatively complete (in the sense of Section 9.4) for executions of $\mathcal{R}$ on a working memory that contains at most one instance of each type.

$$
\begin{aligned}
& \left\{p_{i} \wedge \neg \delta_{i}\right\} r_{i}^{*}\left\{q_{i}\right\} \quad \text { for } i=1, \ldots, n
\end{aligned}
$$

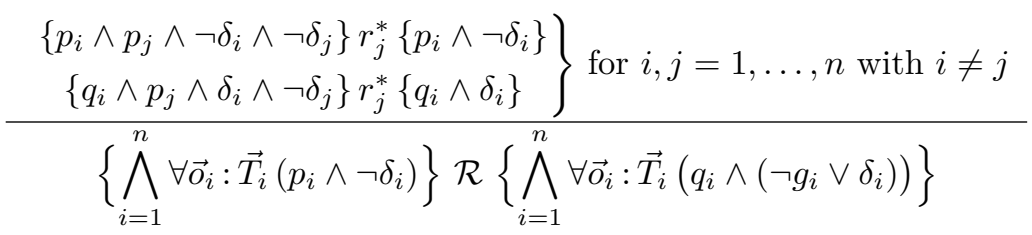

Proof. The soundness of (PR4) is a consequence of Theorem 9.2 stating the soundness of (PR2), and of Lemma 10.4 above.

Its relative completeness results from the relative completeness of (PR2) stated by Theorem 9.4. The relative completeness of (PR2) implies that for any valid global correctness formula $\{p\} \mathcal{R}\{q\}$, there exist eligibility-aware assertions $p_{i}, q_{i}$ for $i=1, \ldots, n$, such that:

(i) The assertions are focused on the corresponding rules, that is, $p_{i}, q_{i} \in$ $\mathcal{F}_{\Delta}\left(r_{i}\right)$. 
(ii) The eligibility-aware correctness formulas $\left\{p_{i} \wedge \neg \delta_{i}\right\} r_{i}^{*}\left\{q_{i}\right\}$ hold.

(iii) They are interference free, with in particular

$$
\left.\begin{array}{c}
\left\{p_{i} \wedge p_{j} \wedge \neg \delta_{i} \wedge \neg \delta_{j}\right\} r_{j}^{*}\left\{p_{i} \wedge \neg \delta_{i}\right\} \\
\left\{q_{i} \wedge p_{j} \wedge \delta_{i} \wedge \neg \delta_{j}\right\} r_{j}^{*}\left\{q_{i} \wedge \delta_{i}\right\}
\end{array}\right\} \text { for } i, j=1, \ldots, n \text { with } i \neq j .
$$

(iv) The correctness formula derived from them by (PR2), which is the same as the one in the conclusion of (PR4), leads to $\{p\} \mathcal{R}\{q\}$.

The first three conditions correspond to the premises of (PR4). Together with the last one, they express the relative completeness of (PR4) for unary rule programs.

Informally, a working memory with at most one instance per type avoids aliasing by a similar mechanism as unary rules. Indeed, unary rules limit the number of objects involved in a rule instance to one. As a result, there cannot be two distinct instances of a rule that update the same object. On the other hand, a working memory with at most one instance per type limits the number of instances of each rule to one. The consequence is that there cannot be two distinct instances of a rule at all.

One can sketch a mapping between the two settings that shows that they are equivalent: given a rule program $\mathcal{R}$ and a working memory $\mathcal{M}$ containing at most one instance of each type, one can build another rule program $\mathcal{R}^{\prime}$ made of unary rules and a working memory $\mathcal{M}^{\prime}$, and vice versa, such as the executions of $\mathcal{R}$ on $\mathcal{M}$ are in bijection with the executions of $\mathcal{R}^{\prime}$ on $\mathcal{M}^{\prime}$.

From $\mathcal{R}$ and $\mathcal{M}$ to $\mathcal{R}^{\prime}$ and $\mathcal{M}^{\prime}$. We consider a rule program $\mathcal{R}=\left\{r_{1}, \ldots, r_{n}\right\}$ and a working memory $\mathcal{M}$, such that for $i=1, \ldots, n$ and for $j=1, \ldots,\left|r_{i}\right|$ one has $r_{i}=\left(\vec{o}_{i}, \vec{T}_{i}, g_{i}, a_{i}\right)$ and $\left|\mathcal{M}\left(T_{j}^{i}\right)\right| \leq 1$.

We introduce a new unifying type $U$ that includes in its definition all the attributes symbols in the definitions of the types appearing in the rules:

$$
\alpha(\mathrm{U}) \stackrel{\text { def }}{=} \bigcup_{\substack{i=1, \ldots, n \\ j=1, \ldots,\left|r_{i}\right|}} \alpha\left(T_{j}^{i}\right) .
$$

This definition benefits from the constraint stated in Section 3.3.2 that type definitions are disjoint.

Since each type $T_{j}^{i}$ has at most one instance, the whole working memory $\mathcal{M}$ can be represented by one instance of $U$. And since all the types have been unified, the sole rule instance of each rule of $\mathcal{R}$ can be mapped onto an instance of an equivalent rule with only one variable of type $U$, on the unique instance of $U$.

From $\mathcal{R}^{\prime}$ and $\mathcal{M}^{\prime}$ to $\mathcal{R}$ and $\mathcal{M}$. We consider a rule program $\mathcal{R}^{\prime}=\left\{r_{1}, \ldots, r_{n}\right\}$ made of unary rules $r_{i}=\left(o_{i}, T_{i}, g_{i}, a_{i}\right)$, and a working memory $\mathcal{M}^{\prime}$ with possibly several instances of each type.

For each $i=1, \ldots, n$, if the working memory contains $m_{i}$ instances of the type $T_{i}$, we define in $\mathcal{T}$ as many copies $T_{1}^{i}, \ldots, T_{m_{i}}^{i}$ of the type, each with only one instance in $\mathcal{M}$, and we define as many copies of the rule $r_{i}$ in $\mathcal{R}$, each with a variable typed by a copy of $T_{i}$, that is, $r_{j}^{i}=\left(o_{j}^{i}, T_{j}^{i}, \sigma_{i j}\left(g_{i}\right), \sigma_{i j}\left(a_{i}\right)\right)$ with the substitution $\sigma_{i j}=\left[o_{j}^{i} / o_{i}\right]\left[f_{j} / f\right]_{f \in \alpha\left(T_{i}\right)}$ for $j=1, \ldots, m_{i}$. 
Example. As an example of a correctness formula that holds on an n-ary rule program when the working memory contains at most one instance of each type, one can use the program and correctness formula proposed in Section 10.1.2, only slightly modified into a two-variables version:

$$
\begin{aligned}
& r_{1}(\mathrm{c}: \text { Cart, } \mathrm{p}: \text { Person }): \text { p.age }>60 \rightarrow \text { c.discount }:=\mathrm{c} \text {.discount }+10 \\
& \mathrm{r}_{2}(\mathrm{c}: \text { Cart, } \mathrm{p}: \text { Person }): \text { p.cat }=\text { Gold } \rightarrow \text { c.discount }:=\mathrm{c} \text {.discount }+15 \\
& \{\forall p: \text { Person }(p . a g e>60 \wedge p . c a t=\text { Gold }) \wedge \forall c: \text { Cart }(\text { c.discount } \leq 30)\} \mathcal{R} \\
& \{\forall c \text { : Cart }(\text { c.discount } \leq 55)\} .
\end{aligned}
$$

The proof of this correctness formula by (PR4) follows the same path as in Section 10.1.2, only with assertions and eligibility ghost variables having an arity of two instead of being unary. However, since the working memory contains at most one instance of each type, no possibility of aliasing needs be considered.

\subsection{Disjointness in Rule Programs}

In an analogy with disjoint parallel programs and the proof rule proposed by Hoare in this case, the present section provides a proof rule for disjoint rule programs made of unary rules. The constraint for rules to be unary is required in addition to disjointness, to obtain a syntactic sufficient condition for interference freedom.

This section also studies disjoint rule programs, for which a compositional proof rule is proposed. This proof rule can in particular be useful in the case of programs implementing decision tables.

\subsubsection{Disjoint Unary Rules}

In [Hoa76], Hoare proposes a proof rule for disjoint parallel programs, that is, parallel programs in which no process ever modifies any variable read by another process. The constraint on the program is strong, but the benefit is a very simple proof rule, as disjointness between processes eliminates any possibility of interference (see for example [AdBO09]).

A similar constraint on the syntax of rules that would eliminate any interference must address the two kinds of interference described in Section 8.1. Combining disjointness (defined just below) and the constraint for rules to be unary gives a sufficient syntactic condition to ensure interference freedom. Intuitively, disjointness addresses cross-rule interference, while unary rules address interference due to aliasing. For that matter, the constraint for rules to be unary may be replaced by the constraint for the working memory to contain at most one instance of each type, as discussed in Section 10.2.

\section{Disjointness}

Definition 10.1. Disjointness is defined as follows:

(i) Change $(r)$ is the set of attributes updated by the action of a rule $r$. 
(ii) The rules $r$ and $r^{\prime}$ are disjoint if no attribute modified by either of the rules occurs in the other rule, that is, Change $(r) \cap \operatorname{Attr}\left(r^{\prime}\right)=\emptyset$ and Change $\left(r^{\prime}\right) \cap \operatorname{Attr}(r)=\emptyset$.

(iii) The rule $r$ is disjoint from the assertion $\varphi$ if no attribute modified by $r$ occurs in $\varphi$, that is, Change $(r) \cap \operatorname{Attr}(\varphi)=\emptyset$.

(iv) The rule programs $\mathcal{R}=\left\{r_{1}, \ldots, r_{n}\right\}$ and $\mathcal{R}^{\prime}=\left\{r_{1}^{\prime}, \ldots, r_{m}^{\prime}\right\}$ are disjoint if for $i=1, \ldots, n$ and $j=1, \ldots, m$ the rules $r_{i}$ and $r_{j}^{\prime}$ are disjoint.

(v) The rule program $\mathcal{R}=\left\{r_{1}, \ldots, r_{n}\right\}$ is disjoint from the set of focused correctness formulas $\left\{p_{i}\right\} r_{i}\left\{q_{i}\right\}$ if for $i, j=1, \ldots, n$ with $i \neq j$, each rule $r_{i}$ is disjoint from $p_{j}$ and from $q_{j}$.

Note that for a rule $r=(\vec{o}, \vec{T}, g, a)$ with $a \equiv o_{1} \cdot f_{r}:=e_{r}$, one has Change $(r)=$ $\left\{f_{r}\right\}$. As a result, the rule $r$ and an assertion $\varphi$ are disjoint if $f_{r} \notin \operatorname{Attr}(\varphi)$.

Example. Consider the three rules below:

$$
\begin{aligned}
& r_{1}(c: \text { Cart, }: \text { Person }): \text { p.age }>21 \rightarrow \text { c.bonus }:=10 \\
& r_{2}(c: \text { Cart, } p: \text { Person }): \text { c.discount }>20 \rightarrow \text { p.cat }:=\text { Gold } \\
& r_{3}(c: \text { Cart, }: \text { Person }): \text { p.age }<50 \rightarrow \text { c.discount }:=15
\end{aligned}
$$

The rule program $\left\{r_{1}, r_{2}\right\}$ is made of disjoint rules, but not the rule program $\left\{r_{2}, r_{3}\right\}$ since Change $\left(r_{3}\right)=\{$ discount $\}$ and $\operatorname{Attr}\left(r_{2}\right)=\{$ cat, discount $\}$.

Consider in addition the following correctness formulas:

$$
\begin{aligned}
\{\text { p.age } & =30\} r_{1}\{\text { c.bonus }=10\} \\
\{\text { c.discount } & =25\} r_{2}\{\text { p.cat }=\text { Gold }\} \\
\{\text { p.age } & =30\} r_{3}\{\text { c.discount }=15\} .
\end{aligned}
$$

The rule program $\left\{r_{1}, r_{2}\right\}$ is disjoint from this set of correctness formulas, but not the rule program $\left\{r_{2}, r_{3}\right\}$ since discount $\in \operatorname{Attr}($ c.discount $=25)$.

\section{A syntactic sufficient condition for interference freedom}

If a rule program is made of unary rules, and is disjoint from a set of valid correctness formulas focused on the rules, then the global correctness formula obtained by combining the individual ones holds. This property is formalized by the following theorem.

Theorem 10.6. Let $\mathcal{R}=\left\{r_{1}, \ldots, r_{n}\right\}$ be a rule program, and for $i=1, \ldots, n$ let $p_{i}, q_{i} \in \mathcal{F}\left(r_{i}\right)$ be assertions focused on the rule $r_{i}$. If $\mathcal{R}$ is made of unary rules, and $\mathcal{R}$ is disjoint from the correctness formulas $\left\{p_{i}\right\} r_{i}\left\{q_{i}\right\}$, then the proof rule below is sound:

$$
\frac{\left\{p_{i}\right\} r_{i}\left\{q_{i}\right\} \quad \text { for } i=1, \ldots, n}{\left\{\bigwedge_{i=1}^{n} \forall o_{i}: T_{i} p_{i}\right\} \mathcal{R}\left\{\bigwedge_{i=1}^{n} \forall o_{i}: T_{i} q_{i}\right\}}
$$

Proof. Assume that the correctness formulas $\left\{p_{i}\right\} r_{i}\left\{q_{i}\right\}$ hold for $i=1, \ldots, n$. Let us prove that under the hypotheses of the theorem, the correctness formulas $\left\{p_{i} \wedge p_{j}\right\} r_{j}\left\{p_{i}\right\}$ and $\left\{q_{i} \wedge p_{j}\right\} r_{j}\left\{q_{i}\right\}$ also hold, for $i, j=1, \ldots, n$ with $i \neq j$. 
We will then derive the conclusion of (PR5) by proof rule (PR1) introduced by Theorem 8.2.

Consider two states $s$ and $s^{\prime}$ and a valuation $\nu$, such that $s \stackrel{\left(r_{j}, \nu\right)}{\longrightarrow} s^{\prime}$. If the rule instance $\left(r_{j}, \nu\right)$ is not applicable in $s$, then the state $s^{\prime}$ is defined as identical to $s$. As a consequence, if $s$ satisfies the precondition of either correctness formula, then $s^{\prime}$ satisfies its conclusion.

If, on the other hand, the rule instance $\left(r_{j}, \nu\right)$ is applicable in $s$, then the state $s^{\prime}$ is defined by the transition assertion for the action of rule $r_{j}$, that is, $\left\langle s, s^{\prime}\right\rangle, \nu \models \hat{a}_{j}$. In particular (see Section 5.3.3), this transition assertion gives that

$$
\left\langle s, s^{\prime}\right\rangle, \nu \models \bigwedge_{f \in \operatorname{Attr} \backslash\left\{f_{r_{j}}\right\}} \forall o\left(o . f^{\prime}=o . f\right) .
$$

The disjointness hypothesis states in particular that $r_{j}$ is disjoint from the correctness formula $\left\{p_{i}\right\} r_{i}\left\{q_{i}\right\}$, that is, the assertions $p_{i}$ and $q_{i}$ do not include any reference to the attribute symbol $f_{r_{j}}$. It results from the transition assertion above that their truth values are the same in $s$ and in $s^{\prime}$. As a consequence, if $s$ satisfies the precondition of either correctness formula, then $s^{\prime}$ satisfies its conclusion.

The premises of proof rule (PR1) are met: its application gives the conclusion of (PR5), which proves the theorem.

As for previous proof rules, we can note that if the sole variables of all the rules in the program have the same type, that is, if $r_{i}=\left(o_{i}, T, g_{i}, a_{i}\right)$ for $i=1, \ldots, n$ and some type name $T$, then the conclusion of the proof rule can be rewritten, and the proof rule reads

$$
\frac{\left\{p_{i}\right\} r_{i}\left\{q_{i}\right\} \quad \text { for } i=1, \ldots, n}{\left\{\forall o: T \bigwedge_{i=1}^{n} p_{i}(o)\right\} \mathcal{R}\left\{\forall o: T \bigwedge_{i=1}^{n} q_{i}(o)\right\}}
$$

The two examples below illustrate that both the disjointness and unary rules constraints are needed for proof rule (PR5) to be sound.

Example. The proof of Proposition 6.2 used the rule program $\mathcal{R}=\{r\}$ made of the only rule below:

$$
r(c: \text { Cart, } p: \text { Person }): \text { p.age }=21 \rightarrow \text { c.value }:=\text { c.value }+10
$$

Since this program is made of only one rule, any disjointness condition is met by construction. However the rule $r$ is not unary.

We noticed that although the correctness formula

$$
\{\text { p.age }=21 \wedge \text { c.value }=0\} r\{\text { c.value }=10\}
$$

holds for the rule $r$, the correctness formula

$$
\{\forall c: \text { Cart } \forall p: \text { Person }(\text { p.age }=21 \wedge \text { c.value }=0)\} \mathcal{R}\{\forall c: \text { Cart }(\text { c.value }=10)\}
$$

which would result from the application of proof rule (PR5), does not hold for the rule program $\mathcal{R}$. Indeed, an execution of the rule program can include the application of several instances of $r$ that share the same cart. The value of this cart is then incremented as many times, and hence exceeds 10 .

Such an aliasing on a shared cart would be made impossible by the constraint for rules to be unary. 
Example. To illustrate the role of disjointness, consider the rule program $\mathcal{R}=$ $\left\{r_{1}, r_{2}\right\}$ made of the two unary rules below, which we used as an example of unary rules on page 112:

$$
\begin{aligned}
& r_{1}(p: \text { Person }): \text { p.age }>60 \rightarrow \text { p.bonus }:=\text { p.bonus }+10 \\
& r_{2}(p: \text { Person }): \text { p.cat }=\text { Gold } \rightarrow \text { p.bonus }:=\text { p.bonus }+15
\end{aligned}
$$

These rules are not disjoint with the following, otherwise valid, correctness formulas:

$$
\begin{aligned}
\{p . \text { age }=70 & \wedge \text { p.bonus }=0\} r_{1}\{p . \text { bonus }=10\} \\
\{p . \text { cat }=\text { Gold } & \wedge \text { p.bonus }=0\} r_{2}\{p . \text { bonus }=15\} .
\end{aligned}
$$

And indeed, the correctness formula

$$
\begin{aligned}
\{\forall p: \text { Person }(p . \text { age }=70 \wedge p . c a t & =\text { Gold } \wedge \text { p.bonus }=0)\} \mathcal{R} \\
& \{\forall p: \text { Person }(\text { p.bonus }=10 \wedge p . \text { bonus }=15)\}
\end{aligned}
$$

which would result from the application of proof rule (PR5) does not hold, since its postcondition is false.

As a final note, we return to the proof rule proposed in Proposition 6.2 for a rule program made of only one rule. This proof rule did not hold in general, however it does in the case of unary rules, as a consequence of Theorem 10.6:

$$
\frac{\{p\} r\{q\}}{\{\forall o: T p\} \mathcal{R}\{\forall o: T q\}} \quad \text { where } \mathcal{R}=\{r\} \text { and } r \text { is unary }
$$

\subsubsection{Disjoint Rule Programs}

Proof rule (PR5) just exposed applies to rule programs and correctness formulas where each rule in the program is disjoint from the correctness formulas focused on all other rules. However, another occurrence of disjointness in rule programs is of interest, namely a set of rules that can be partitioned into disjoint groups. In such a case, a correctness formula for the whole set of rules can be derived from correctness formulas for each group.

Theorem 10.7. Let $\mathcal{R}_{1}$ and $\mathcal{R}_{2}$ be two rule programs made of rules of arbitrary arities, and let $p_{1}, q_{1}, p_{2}, q_{2} \in \mathcal{F}(\emptyset)$ be global assertions, such that:

(i) $\mathcal{R}_{1}$ and $\mathcal{R}_{2}$ are disjoint;

(ii) every rule in $\mathcal{R}_{1}$ is disjoint from $p_{2}$ and from $q_{2}$;

(iii) every rule in $\mathcal{R}_{2}$ is disjoint from $p_{1}$ and from $q_{1}$.

Then the following proof rule is sound:

$$
\frac{\left\{p_{1}\right\} \mathcal{R}_{1}\left\{q_{1}\right\} \quad\left\{p_{2}\right\} \mathcal{R}_{2}\left\{q_{2}\right\}}{\left\{p_{1} \wedge p_{2}\right\} \mathcal{R}_{1} \cup \mathcal{R}_{2}\left\{q_{1} \wedge q_{2}\right\}} .
$$

Proof. Consider an execution of the rule program $\mathcal{R}_{1} \cup \mathcal{R}_{2}$. It is composed of applications of instances of rules taken either in $\mathcal{R}_{1}$ or in $\mathcal{R}_{2}$. Due to the disjointness hypotheses, each application of an instance of a rule from $\mathcal{R}_{1}$ updates 
an attribute that occurs neither in the rules of $\mathcal{R}_{2}$, nor in $p_{2}$ or $q_{2}$; and vice versa.

Any execution of $\mathcal{R}_{1} \cup \mathcal{R}_{2}$ is therefore equivalent (in the sense given below) to an execution where all the instances of rules from $\mathcal{R}_{1}$ are applied before all the instances of rules from $\mathcal{R}_{2}$, with the respective orders of instances from the same rule program left unchanged. These executions are equivalent from the viewpoint of the updates performed by each rule instance application, and of their effects on the applicability of other rule instances. As a consequence, they satisfy the same global correctness formulas.

If such a reordered execution is performed from a state that satisfies $p_{1} \wedge p_{2}$, then when it reaches the state where all the instances of rules from $\mathcal{R}_{1}$ have been applied, this state satisfies $q_{1} \wedge p_{2}$. All the instances of rules from $\mathcal{R}_{2}$ are then applied to get to the final state, which satisfies $q_{1} \wedge q_{2}$.

As a result, the correctness formula $\left\{p_{1} \wedge p_{2}\right\} \mathcal{R}_{1} \cup \mathcal{R}_{2}\left\{q_{1} \wedge q_{2}\right\}$ holds, which proves the soundness of the proof rule.

Proof rule (PR7) is particularly interesting the case of rule programs structured as disjoint decision tables. A decision table [Ros03] includes a set of condition columns and one decision column; each row in the table maps a configuration of the condition attributes onto a value to be assigned to the decision attribute. A decision table can be naturally implemented by a set of rules that all involve the condition attributes in their guards, and all update the decision attribute in their actions. Fig. 10.1 shows an example of a decision table, and of its implementation as a rule program.

\begin{tabular}{|c|c|c||c|}
\hline \multicolumn{2}{|c|}{ p.age } & \multicolumn{1}{|c|}{} & \\
\hline $\min$ & $\max$ & p.cat & c.discount \\
\hline \multirow{2}{*}{0} & \multirow{3}{*}{20} & Silver & 0 \\
\cline { 3 - 4 } & & Gold & 5 \\
\cline { 3 - 4 } & & Plat & 10 \\
\hline \multirow{3}{*}{21} & \multirow{3}{*}{60} & Silver & 10 \\
\cline { 3 - 4 } & & Gold & 15 \\
\cline { 3 - 4 } & & Plat & 20 \\
\hline \multirow{3}{*}{61} & \multirow{3}{*}{$\infty$} & Silver & 15 \\
\cline { 3 - 4 } & & Gold & 20 \\
\cline { 3 - 4 } & & Plat & 25 \\
\hline
\end{tabular}

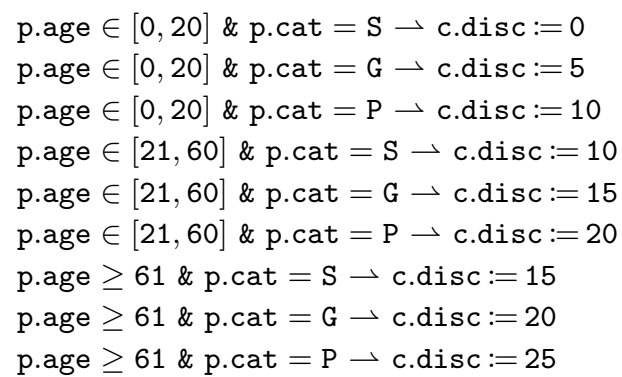

Figure 10.1: A decision table and its implementation as a rule program.

In a set of rules implementing a decision table, the rules cannot be disjoint from (non-trivial) correctness formulas focused on the other rules. However, a rule program made of disjoint decision tables will benefit from proof rule (PR7) to derive a global correctness formula for the rule program from the individual correctness formulas for the decision tables. The latter being proven with the other proof rules presented in Part III. 


\section{Chapter 11}

\section{Conclusion and Future Work}

In this thesis we have studied rule programs as handled by Business Rules Management Systems. In Part I we have formally described rules, rule programs, and the meaning of their executions. Then in Part II we have introduced a Hoare logic so that correctness properties can be expressed about either rules or rule programs. Finally in Part III we have proposed a compositionial verification method to derive correctness formulas for rule programs from individual correctness formulas for the rules in the program.

Throughout this work, we have dealt with both local and global aspects. Rules, and furthermore rule instances, have a local reach in that they involve only a handful of objects. Rule programs have a global reach, as they handle a whole working memory. Yet, the interactions between rules, as well as between rules and objects, blur the frontier between local and global scopes. This motivated the search for a verification method to turn local knowledge about the rules into global information about the rule program.

In this chapter we review our contribution from the standpoint of this tension between local and global approaches to rule programs as handled by Business Rules Management Systems. We also give indications for possible extensions of the present work.

\subsection{Modeling an Industrial Product}

An industrial product, in our case Business Rules Management Systems, has a de-facto approach to definitions. Elaborating a model for such a product is reminiscent of the work performed by knowledge engineers with business experts in eliciting a domain knowledge. Proposing a description on which to base the development of formal tools includes making choices in order not to be overwhelmed with peculiarities of the industrial product, while not losing the specificities that make it an interesting subject of study.

The choices we made in defining a rule language in Chapters 3 and 4 included simplifications with respect to the rule languages found in BRMS. Among the aspects that were discarded, but which could be the subject of future work, is the ability for a rule to perform more than an assignment in its action: for 
example add objects into the working memory and remove objects from it, or the whole range of imperative programming constructs. Also, our rules only check for the existence in working memory of object that satisfy the conditions included in their guards. Negative patterns, such as there is no person older than 60 , could be added to the guards of rules, and more generally aggregations over collections of objects. Similarly, our assertion language could be enriched with constructs expressing the number of objects that satisfy a given condition, with a possible extension towards counter abstraction [ET99, PXZ02, BMWK09]. Finally, rules in BRMS make extensive use, both in their guards and in their actions, of functions that cannot be analyzed because their code is not accessible. Examples include Java methods. We do not cover them in our formalism; this could be done for instance by including uninterpreted functions with equality in the fixed theory $\Theta$ that represents the application data types (see Section 3.1).

In spite of these simplifications, important aspects of rules as handled by BRMS were retained. These include for example the description of other semantics of rule engines than the classical Rete-based one (see Chapter 5). Also, the rules in our language apply to tuples of objects. As a corollary, we had to take the risk of aliasing into account when formalizing the updates performed in the rule actions. That is: if a rule modifies the color of the car of John, does it affect the car of Mary? This point materializes in Sections 4.4, 5.3, 8.1.2, 9.2.2. This salient feature of rule programs in BRMS had to be present for our formalization to be relevant.

\subsection{Local vs. Global: What Is a State?}

When writing a rule, or when reasoning about a rule, the world is perceived from a local viewpoint: it includes the objects handled by the rule, either to evaluate its guard or to execute its action. In contrast, when writing a rule program or reasoning about it, a whole working memory is considered, and the world consists in transitions between states of this working memory.

In Chapter 4 the definition of a state is introduced, as a first-order logic structure that interprets each attribute symbol by a function from objects to values of the domain. With this definition, a state is further used to describe three scopes:

(i) When reasoning about the executions of a rule program on a working memory, as in Chapter 5, a state is considered from a global viewpoint. It provides all the information to describe the realm of a rule program execution, namely the values of the attributes of the objects in the working memory (a finite set of objects, see Definition 5.4).

(ii) When considering a rule in isolation, objects are accessed through a tuple of variables (see Section 3.5). The objects themselves are not fixed; only their number and their types are. From this viewpoint, the rule scope is both limited by, and extends to, all the tuples of objects that can be formed from the working memory to comply with this number and these types (see Definition 5.3).

(iii) From the local viewpoint of a rule instance (a rule plus a tuple of objects, see Definition 5.6), the objects are fixed. The scope is then described by the portion of a state limited to the objects contained in the instance. 
Between these three scopes, a tension results, which correspond to different ways of thinking of a rule program. This variety can cause misunderstandings between the stakeholders in a Business Rules Management System. A precise definition of the behavior of a rule program as handled by a BRMS, as given in Part I, was therefore needed.

\subsection{Are Rule Programs Magical?}

The tension between various scopes reflects the opposition between authoring rules and executing rule programs.

Due to its declarative nature, a rule program is written with the rule scope in mind. For rule authors, the objects to consider are the ones that could match the rule, that is, the object tuples well-typed for the rule.

On the other hand, the execution of a rule program is considered either in the global scope of a whole working memory, or in the most local scope of the rule instances applied.

Rule authors and rule engines thus have different approaches to a rule program. A consequence is that the authors of rule programs do not (and cannot) always take into account the interference cases that might occur at execution time between the instances of the rules that compose the program.

Furthermore, these interference cases require some training to get used to imagining them. Indeed, not only can they affect the values of attributes in the successive states during the execution, but they can also affect whether a rule (actually some of its instances) can be executed or not. In this, rule programs differ from parallel programs. In the latter, interference between processes can affect the control of the program only through mechanisms that are explicit in the program text. In rule programs however, rule instance eligibility plays a role at the rule engine level and is not visible in the text of rules. Examples such as the one used in the proof of Proposition 7.1, or those exposed in Sections 5.6.2 or 10.1.1, illustrate this difference.

The potentially complex impact of interference cases in rule programs probably contributes to their reputation of having unpredictable executions. As some clever marketing recently suggested, "when something exceeds your ability to understand how it works, it sort of becomes magical." [1] However science has taught us that computer programs are not magical, but instead can be formally described and reasoned about. Hence the proposal of a formal tooling for rule programs.

Chapter 5 exposes the operational semantics of a rule program. This presentation introduces rule instances as building blocks of an execution, and an abstract machine whose configurations and the transitions between them orchestrate the applications of rule instances. The process by which a rule engine selects the rule instance to apply among all the applicable and eligible ones is reified as the selection strategy. The eligibility strategy represents the process by which the engine defines which rule instances are eligible and which are not. These two strategies are parameters to the abstract machine that defines the operational semantics given to a rule program by a rule engine.

Isolating the selection and eligibility strategies allows us to give a description not only of the Rete algorithm, but also of the alternatives to Rete that have been introduced in Business Rule Management Systems in the past decade 
[IBM11, Red10, Fai07]. The verification method described in Part III was designed specifically for the so-called one-shot strategy (see Section 6.1.1). An extension of our work could include the design of similar methods for other strategies. Furthermore, the properties of strategies and their influence on properties of the rule programs, in particular termination, could be investigated [PR04, PR05, CPR07].

\subsection{From Local to Global}

Part I defines assertions, used to express properties of states and of transitions between states. Based on these assertions, Part II defines correctness formulas to express properties of the execution of rules and of rule programs.

Correctness formulas are of the classical form $\{p\} S\{q\}$, and are given in Chapter 6 a precise (and different) meaning when $S$ is a rule or a rule program. This need for a distinct definition on rules and on rule programs is another manifestation of the tension between local and global scopes, between properties of executions of a rule and properties of executions of a rule program.

Part III resolves this tension with a compositional verification method that builds a link between the local reach of a rule and the global reach of a rule program. The search for this verification method is motivated by the fact that a rule program can in general not be simulated by a parallel program. This discards the option of reusing a verification method for parallel programs, such as the one introduced by Owicki and Gries [OG76].

Our verification method is built on a sound and relatively complete proof rule to derive a correctness formula for a rule program from individual correctness formulas for the rules in the program. This proof rule is given in Chapter 9, where its soundness and relative completeness are also proven. In addition, specialized proof rules are given for particular classes of rule programs, which can benefit from simplifications. One of them is given in Chapter 8 , and serves as a presentation of the foundations of our verification method. The other ones are given in Chapter 10. All of the proof rules are illustrated in detail on examples.

In Section 9.4, the relative completeness of the most general proof rule is shown, meaning that any valid correctness formula for a rule program can be derived from a set of valid, interference free correctness formulas for the rules of the program. However, the completeness proof is not constructive and no algorithm is provided to find the individual interference free correctness formulasalthough practical lemmas are given, which help checking of the premises of the proof rules or splitting the rule program into independent chunks. Studying whether such an algorithm always exists, and furthermore proposing one, is a natural direction for a follow-up to this work.

\subsection{Discussion}

In this thesis we have proposed a set of formal tools for the expression and verification of correctness properties of rule programs as handled by Business Rules Management Systems.

Our contribution includes a formal, logic-based description of the behavior of rule programs in BRMS, a Hoare logic, and a sound and relatively complete 
compositional verification method for these rule programs.

These tools can be used to express and verify global correctness properties of rule programs, based on local knowledge about the individual rules in the program.

With this contribution, our objective is to assist authors of rule programs and developers of BRMS in precisely understanding the meaning of the programs they write or handle, and in proving properties of their executions. In Appendix A, we validate the adequacy of our formal framework to express the Rule Static Analysis features of the IBM Websphere Operational Decision Management product. Our contribution could therefore provide a basis for further developments in BRMS features.

Further research can be proposed as an extension of this work. On the theoretical side, it would be interesting to investigate how our work could be enriched by incorporating results from term-rewriting systems [KB70, SS94, SS99] to study the confluence of rule programs; from shape analysis [JM79, CWZ90] when relations between objects matter; from such abstractions as predicate abstraction [GS97, LBC03] or counter abstraction [ET99, PXZ02] to provide more expression power to the local and global states involved in a rule program execution, especially for execution strategies that do not guarantee termination. On the practical side, extending our formal framework with uninterpreted functions and proposing an algorithm to support the application of our proof rules are probably the main enablers for a direct use of our work in a BRMS. 


\section{Appendix A}

\section{Verification in an Industrial Business Rules Management System}

In this chapter, we apply our results to an existing industrial Business Rules Management System, that is, the commercial BRMS sold by IBM under the name IBM Websphere Operational Decision Management [IBM11, 5].

IBM Websphere Operational Decision Management contains several features related to the verification of properties of the rule programs it handles. These features are collectively named Rule Static Analysis. We review them below, and we provide for each of them a description of the feature as offered in the product, and a formalization with the tools exposed in the previous chapters.

This review is an opportunity to demonstrate the adequacy of our formal framework, and to outline how our work could contribute to expand these features. More generally, the task of providing a formal basis for such features is a cornerstone to an agreement on their definitions, for example between product marketing and development teams. In the present case, this is made possible by the introduction of such concepts as states, rule applicability, rule execution relation, or correctness formulas. We start the chapter with a reminder on these concepts.

The Rule Static Analysis features have been developed by a team within ILOG (now IBM) that I have been leading, and that included Ulrich Junker and Michel Leconte. Credit must be given to them for their share in the design and implementation work, and to Steve Demuth for his insight into the subject. The formalization exposed in this chapter leverages our collaboration.

\section{A.1 Preliminaries}

\section{A.1.1 Rule Static Analysis Features}

The Rule Static Analysis features in IBM Websphere Operational Decision Management range from program understanding to lightweight program verification. The latter aims at detecting coding or design errors in the rule program, while 
the former assists the user in understanding or navigating the rule program by presenting rules that match various queries.

The present chapter reviews the Rule Static Analysis features by topic. It first addresses the features related to the applicability of rules, then the ones that analyze the impacts of rule execution. The features covering redundancy, confluence, and completeness are then exposed. Finally it describes a feature that implements a specialized form of safety property checking.

These features are already useful to the users of the product, however none considers the execution of a whole rule program. Some consider the consecutive execution of two rule instances, but none go so far as to have to take eligibility into account. This limitation was a motivation for the work exposed in this thesis, with the investigation of techniques to derive global correctness information for a rule program from local knowledge on each rule.

From an architectural standpoint, the Rule Static Analysis features are provided as a set of services within the IBM Websphere Operational Decision Management product. Their implementation relies on a logic engine that is capable of reasoning on assertions and rules. In contrast with the execution engine, this logic engine can symbolically perform such operations as assertion assessment or rule execution, even when part of the object attributes have no concrete values. To this end, the logic engine relies in turn on a constraint solver that was adapted for program analysis purposes $\left[\mathrm{LB} 06, \mathrm{AKBJ}^{+} 07\right.$, BL10].

\section{A.1.2 Notations}

All the concepts and notations used in this chapter have been introduced in the previous ones. We review them quickly here, with references to their original definitions.

An assertion is a first-order, flat, possibly quantified formula (Section 3.3). A rule $r$ is defined in Section 3.5 as $r=(\vec{o}, \vec{T}, g, a)$, where:

(i) $\vec{o}=\left(o_{1}, \ldots, o_{m}\right)$ is a tuple of variables, called the rule variables.

(ii) $\vec{T}=\left(T_{1}, \ldots, T_{m}\right)$ is a tuple of type names; together with the rule variables they define the rule type constraint $(\vec{o}: \vec{T})$, reminded below.

(iii) $g$ is a non-quantified assertion in the rule variables, called the guard of the rule.

(iv) $a$ is an assignment to some attribute of the object held by rule variable $o_{1}$; its called the action of the rule.

A type constraint is a partial function that maps variables onto type names (see Section 3.3.2). In the context of a rule $r=(\vec{o}, \vec{T}, g, a)$, the notation $(\vec{o}: \vec{T})$ refers to the type constraint $\tau$ such that $\tau\left(o_{j}\right)=T_{j}$ for $j=1, \ldots, m$.

A valuation is a function that maps variables onto objects (Section 4.1.2). A state is a first-order logic structure; it interprets each attribute symbol $f$ as a function, noted $f^{s}$, that provides a value for each object (Section 4.1.5).

An assertion is given a truth value by a state and a valuation. For example, a valuation $\nu$ such that $\nu(p)=$ Alice and a state $s$ such that $a g e^{s}$ (Alice) $=30$ make the assertion p.age $>21$ valid. We then note $s, \nu \models$ p.age $>21$.

Section 5.2 defines the working memory as the finite set of objects matched by rules. It also introduces the concepts of a state compliant with the type 
system, and of a valuation well-typed for a given type constraint. In the present chapter, we only consider states that are compliant with the type system, and valuations that are well-typed for the type constraints inherited from the rules at hand, and we will thus not mention it.

The execution of rules is defined in Section 5.3, by means of rule instances, as follows.

(i) An instance of a rule $r=(\vec{o}, \vec{T}, g, a)$ on a valuation $\nu$ is the $(m+1)$-tuple $\left(r, O_{1}, \ldots, O_{m}\right)$. In this tuple, each $O_{j}$ is an object that is an instance of the type $T_{j}$, and is the image of the rule variable $o_{j}$ by the valuation $\nu$, that is, $O_{j}=\nu\left(o_{j}\right)$ for $j=1, \ldots, m$. The rule instance is also noted $(r, \nu)$.

(ii) Given a rule $r=(\vec{o}, \vec{T}, g, a)$ and a valuation $\nu$, the rule instance $(r, \nu)$ is applicable in a state $s$ if one has $s, \nu \models g$.

(iii) When an applicable rule instance $(r, \nu)$ is applied in a state $s$, it produces a new state $s^{\prime}$. The new state results from the execution of the rule action on the objects in the rule instance, as described in Section 5.3.3.

(iv) Finally, the execution of a rule instance $(r, \nu)$ from a state $s$ to a state $s^{\prime}$ is noted $s \stackrel{(r, \nu)}{\longrightarrow} s^{\prime}$. The state $s^{\prime}$ is defined as the result of the application of $(r, \nu)$ in $s$ if the rule instance is applicable in this state; it is defined as identical to $s$ otherwise.

The execution of a rule $r$ is a relation between states that gathers the executions of all the possible instances of the rule. This relation is noted $\stackrel{r}{\rightarrow}$ and is defined as follows: given two states $s$ and $s^{\prime}$, we have $s \stackrel{r}{\rightarrow} s^{\prime}$ if there exists an instance $(r, \nu)$ of the rule, such that $s \stackrel{(r, \nu)}{\longrightarrow} s^{\prime}$.

In the present chapter, we also use correctness formulas for rules, which are defined in Section 6.2. A correctness formula for a rule $r$ is of the form $\{p\} r\{q\}$, where $p$ and $q$ are assertions, respectively called the pre- and postconditions of the correctness formula. The semantics of the correctness formula is the classical one: it holds if any execution of an instance of $r$ that starts in a state that satisfies $p$, ends in a state that satisfies $q$. That is:

$$
\{p\} r\{q\} \text { holds } \quad \text { iff }\left\{\begin{array}{l}
\text { for any states } s \text { and } s^{\prime} \text {, and any valuation } \nu, \\
\text { such that } \\
s, \nu \models p \text { and } s \stackrel{(r, \nu)}{\longrightarrow} s^{\prime} \\
\text { one has } s^{\prime}, \nu \models q .
\end{array}\right.
$$

\section{A.2 Rule Applicability}

As previously mentioned, we start the review of the Rule Static Analysis features with those related to the applicability of rules. The next sections cover the features related to the effects of rule execution, to redundancy, confluence, completeness, and finally to safety property checking.

For each of them, we first give an informal, business-oriented description of the feature and of its purpose, with an example. Then we proceed to the formal presentation of the feature, based on the formal framework introduced in Chapters 3 to 6 of this thesis. When relevant, we conclude with a discussion. 


\section{A.2.1 Rule may apply when...}

This Rule Static Analysis feature is typically used when a rule-based application is audited. In such a situation, the auditor wants to verify which rules will be considered for application by the rule engine in some given situations.

The "rule may apply when" feature allows the auditor to check whether the guard of a rule is compatible with an assertion that describes the situation considered. When used as a query, the feature yields all the rules whose applicability is not prevented by the assertion.

Example. Consider the three rules below:

$$
\begin{aligned}
& r_{1}(p: \text { Person }): \text { p.age } \leq 30 \rightarrow \text { p.bonus }:=10 \\
& r_{2}(p: \text { Person }): \text { p.age }>30 \rightarrow \text { p.bonus }:=15 \\
& r_{3}(p: \text { Person }): \text { p.cat }=\text { Gold } \rightarrow \text { p.bonus }:=\text { p.bonus }+5
\end{aligned}
$$

When asking for the rules that "may apply when p.age $\geq 60$ ", the user will be answered with the rules $r_{2}$ and $r_{3}$. Indeed, the guard of rule $r_{1}$ is p.age $\leq 30$, which cannot be valid in any context where p.age $\geq 60$. On the other hand, the fact that $p$.age $\geq 60$ does not prevent the guard of $r_{3}$, that is, $p$.cat $=$ Gold, from being valid.

Using the formal framework introduced in Part I, we can formalize the feature as follows. For this specific feature, we present the formalization in two steps. The first step addresses the intuitive description of the feature as requiring compatibility between the query and the rule applicability. It also introduces the concepts participating in the formal definition of the feature. The second step refines the first formalization to address pragmatic constraints that come from the object-oriented nature of our rule programs.

The "rule may apply when" feature checks whether the guard of a rule is compatible with a given situation. Let us note $r=(\vec{o}, \vec{T}, g, a)$ the rule under consideration. In the example above, $r$ would successively represent each of $r_{1}$, $r_{2}$, and $r_{3}$. Let us also represent the situation described by the query with an assertion $\varphi$. In the example, this would give $\varphi \equiv$ p.age $\geq 60$.

Stating that the query assertion $\varphi$ is compatible with the applicability of the rule $r$ means that an instance of $r$ can be found, that is applicable in a state where $\varphi$ holds. Choosing an instance of $r$ consists in choosing an object for each of the rule variables $\vec{o}=\left(o_{1}, \ldots, o_{m}\right)$ of $r$ : this amounts to choosing a valuation. In the example above, the rule $r$ has only one rule variable $p$, so all valuations that map $p$ to the same object provide the same rule instance.

Once an instance of $r$ is chosen through the choice of a valuation $\nu$, it is applicable in a state $s$ if the rule guard holds in this state and under this valuation. That is, if one has $s, \nu \models g$. The description of the feature requires the assertion $\varphi$ to also hold. One can therefore propose the following formalization:

$$
\text { " } r \text { may apply when } \varphi \text { " }
$$

if

there exist a state $s$ and valuation $\nu$, such that

$$
s, \nu \models g \wedge \varphi \text {. }
$$


As mentioned earlier, this formalization is not correct in all cases. To perceive this, one has to take into account the remark of Section 3.5.1, which noted that the rule variable names should be unique. In other words, the assertion $\varphi$ that represents the query and the rule $r$ must have distinct variables.

Let us thus keep the notation $r=(\vec{o}, \vec{T}, g, a)$ for the rule being considered, with $\vec{o}=\left(o_{1}, \ldots, o_{m}\right)$. And let us now note $x_{1}, \ldots, x_{n}$ the free variables in $\varphi$. In our running example, this gives $\varphi \equiv x$.age $\geq 60$. With this correct notation, the formalization above would give that " $r_{1}$ may apply when $\varphi$ ", which is not wanted. Indeed, the formula $g \wedge \varphi$ is made valid by a valuation $\nu$ that maps $p$ and $x$ onto distinct objects, and a state $s$ that gives ages of 20 and 60 to $\nu(p)$ and $\nu(x)$, respectively.

To avoid this, we add a constraint on the valuation: the objects considered in the query and in the rule instance must not be totally disjoint, if the type constraints permit. Let us hence note $S_{1}, \ldots, S_{n}$ the respective types of the assertion variables $x_{1}, \ldots, x_{n}$, and let us define the set $U$ of common variable types:

$$
U \stackrel{\text { def }}{=}\left\{S_{1}, \ldots, S_{n}\right\} \cap\left\{T_{1}, \ldots, T_{m}\right\}
$$

Then either the variables in the query have completely disjoint types from the variables in the rule, and we consider that the rule "may apply", as nothing prevents it from doing so. Or the variable types are to some degree compatible, and the valuation must not map the variables onto totally distinct objects. This gives the following, final formalization of the feature:

$$
\text { " } r \text { may apply when } \varphi \text { " }
$$

if

there exist a state $s$ and valuation $\nu$, such that

$$
\begin{aligned}
& U=\emptyset \quad \text { or } \quad\left\{\nu\left(x_{i}\right) \mid S_{i} \in U, 1 \leq i \leq n\right\} \cap\left\{\nu\left(o_{j}\right) \mid T_{j} \in U, 1 \leq j \leq m\right\} \neq \emptyset \\
& s, \nu \models g \wedge \varphi .
\end{aligned}
$$

\section{A.2.2 Rule is never applicable}

This feature may be seen as a complement to the previous one, although historically it came first. Its purpose is to detect and report rules whose guard is unsatisfiable.

Example. The rule below is "never applicable":

$$
r(p: \text { Person }): \text { p.age } \leq 20 \& \text { p.age } \geq 80 \rightarrow \text { p.bonus }:=0
$$

The author of this rule most probably meant it to apply in cases where the person is below 20 or above 80 .

"Never applicable" rules usually occur because of typing errors, or when the rule author uses a very complex combination of conjunctions and disjunctions, and is finally mistaken about its meaning. This feature can also reveal a misunderstanding between the business users who authored the rules, and the software engineers who designed the business rule language. Indeed, it is the translation into the technical rule language that is analyzed, and ultimately executed. 
The feature is formalized as follows. Considering a rule $r=(\vec{o}, \vec{T}, g, a)$, we say that

$$
\text { " } r \text { is never applicable" }
$$

if

$$
\text { for any state } s \text { and valuation } \nu \text { one has } \quad s, \nu \models \neg g
$$

or, equivalently, if

$$
\text { for any state } s \text { one has } s \models \forall \vec{o}: \vec{T}(\neg g) \text {. }
$$

\section{A.3 Impact Analysis}

Rule Static Analysis features in the Impact Analysis category aim at exhibiting the consequences of a state transition, such as when a rule is executed, on the applicability of rules.

\section{A.3.1 Rule may lead to a state where...}

This feature focuses on transitions from a state where a given condition does not hold, to a state where it holds. It allows the user to check whether a given rule can cause such a transition. When used as a query, it yields all rules, some executions of which will cause such a transition to occur.

Example. Consider the rules below:

$$
\begin{aligned}
& r_{1}(p: \text { Person }): \text { p.age } \leq 25 \rightarrow \text { p.bonus }:=30 \\
& r_{2}(p: \text { Person }): \text { p.age } \leq 25 \rightarrow \text { p.cat }:=\text { Gold } \\
& r_{3}(p: \text { Person }): \text { p.bonus }>50 \rightarrow \text { p.bonus }:=50
\end{aligned}
$$

When asking for the rules that "may lead to a state where p.bonus $\geq 20$ ", the user will be answered with the rule $r_{1}$. Indeed, when executed from a state where $p$.bonus $<20$, rule $r_{1}$ will cause a transition to a state where $p$.bonus $=30 \geq 20$, provided p.age $\leq 25$ holds in the initial state, which is possible.

In contrast, rules $r_{2}$ and $r_{3}$ will not be part of the answer to this query. For both rules the reason is the same: although one can find executions of the rule where the final state satisfies p.bonus $\geq 20$, the rule cannot be credited for this. Indeed, for $r_{2}$ as for $r_{3}$, when the rule is executed from a state where $p$.bonus $<20$, the execution does not result in a state where p.bonus $\geq 20$.

With $r_{2}$ this is due to the fact that the rule does not update the bonus attribute. With $r_{3}$ this is due to the fact that, when p.bonus $<20$, the rule is not applicable, and hence its execution leaves the system state unchanged, as defined on page 56 and reminded on page 181. Note that the non-skip executions of $r_{3}$ do update the bonus attribute, furthermore to a value greater than 20. However these executions start in a state where it already holds: $r_{3}$ cannot be credited for p.bonus $\geq 20$ holding in the final state.

The feature can be formalized as follows. Considering a rule $r=(\vec{o}, \vec{T}, g, a)$ and an assertion $\varphi$ that represents the query, the rule

$$
\text { " } r \text { may lead to a state where } \varphi "
$$


if

there exist two states $s$ and $s^{\prime}$ and a valuation $\nu$, such that

$$
s, \nu \models \neg \varphi \quad \text { and } \quad s \stackrel{(r, \nu)}{\longrightarrow} s^{\prime} \quad \text { and } \quad s^{\prime}, \nu \models \varphi \text {. }
$$

Note that these conditions have two direct implications. On the one hand, they imply that $s, \nu \models g$. Otherwise, the execution of $(r, \nu)$ would be a skip, and hence $s^{\prime}=s$, which makes the transition from $s, \nu \models \neg \varphi$ to $s^{\prime}, \nu \models \varphi$ impossible. On the other hand, they imply that $\varphi$ contains an occurrence of the attribute updated in the action of $r$. Otherwise, the execution of $(r, \nu)$ would not affect $\varphi$, as noted in Section 5.3.3. These remarks can serve as hints to optimize an implementation of the feature.

More importantly, this feature cannot be formalized by $\{\neg \varphi\} r\{\varphi\}$ nor by $\{g \wedge \neg \varphi\} r\{\varphi\}$. Indeed, these correctness formulas would mean that any execution, resp. any non-skip execution, of $r$ from a state where $\varphi$ does not hold results in a state where it holds, whereas the feature only requires that at least one such execution exists. Consider for example the rule $r_{3}$ below:

$r_{3}(p:$ Person $):$ p.age $\leq 25 \&$ p.bonus $<15 \rightarrow$ p.bonus $:=$ p.bonus +10

This rule "may lead to a state where p.bonus $\geq 20$ ": when executed for example from a state where the age is 21 and the bonus is 12 , it leads to a bonus of 22 . However, some executions of this rule that contradict both correctness formulas

$$
\begin{array}{r}
\{\text { p.bonus }<20\} r_{3}\{\text { p.bonus } \geq 20\} \\
\{\text { p.age } \leq 25 \wedge \text { p.bonus }<15\} r_{3}\{\text { p.bonus } \geq 20\} .
\end{array}
$$

For example, consider an execution from a state where the age is 21 and the bonus is 5 . This state satisfies the rule guard and the negation of the query, and hence the precondition of either correctness formula. However, the execution of the rule from this state results in a state where the bonus is 15 . As a consequence, the postconditions of the correctness formulas do not hold in this resulting state.

This Rule Static Analysis feature is used to find the rules whose execution can end in the state described by the query. Although this information is useful, it is only partial, as one is not only interested in the last rule leading to the considered state, but also in the rules that were executed before. In addition, the initial state causing the rule executions that led to the considered state is interesting as well. Furthermore, as discussed for the "unsafe execution" feature in Section A.6.1 below, even if one rule may lead to a state where some (safety) property is violated, the execution of subsequent rules might correct this and the final state of the rule program as a whole might be safe. However, a feature retrieving the rule program executions that end in a given state would require taking eligibility into account, which is out of the current scope of the Rule Static Analysis features of IBM Websphere Operational Decision Management.

\section{A.3.2 Rule may enable...}

This feature focuses on transitions from a state where a given rule is not applicable, to a state where it is. It can be seen as an extension of the "may lead to a state where..." feature just described, where the query assertion is intensionally given by the applicability condition of a rule. 
Example. Consider the rules below:

$$
\begin{aligned}
& r_{1}(p: \text { Person }): \text { p.age } \leq 25 \rightarrow \text { p.bonus }:=30 \\
& r_{2}(p: \text { Person }): \text { p.bonus }>20 \rightarrow \text { p.cat }:=\text { Gold }
\end{aligned}
$$

The first rule "may enable" the second one because it is possible to find an execution of $r_{1}$ in which rule $r_{2}$ changes from being inapplicable to being applicable. An example of such an execution is from a state where the age is 20 and the bonus is 10. In this state, $r_{2}$ is not applicable. However, the execution of $r_{1}$ results in a state where the bonus is 30 , and hence $r_{2}$ is applicable. that

Considering two rules $r_{1}=\left(\vec{o}_{1}, \vec{T}_{1}, g_{1}, a_{1}\right)$ and $r_{2}=\left(\vec{o}_{2}, \vec{T}_{2}, g_{2}, a_{2}\right)$, we say

$$
\text { " } r_{1} \text { may enable } r_{2} "
$$

if

there exist two states $s$ and $s^{\prime}$ and a valuation $\nu$, such that

$$
s, \nu \models \neg g_{2} \quad \text { and } \quad s \stackrel{\left(r_{1}, \nu\right)}{\longrightarrow} s^{\prime} \quad \text { and } \quad s^{\prime}, \nu \models g_{2} .
$$

Here again, the above implies that $s, \nu \models g_{1}$ and that the attribute updated in $r_{1}$ occurs in $g_{2}$.

A symmetric feature exists, worded " $r_{1}$ may disable $r_{2}$ ". This feature means that there is an execution of $r_{1}$ by which rule $r_{2}$ changes from being applicable to being inapplicable. That is,

$$
\text { " } r_{1} \text { may disable } r_{2} "
$$

if

there exist two states $s$ and $s^{\prime}$ and a valuation $\nu$, such that

$$
s, \nu \models g_{2} \quad \text { and } \quad s \stackrel{\left(r_{1}, \nu\right)}{\longrightarrow} s^{\prime} \quad \text { and } \quad s^{\prime}, \nu \models \neg g_{2} .
$$

These two features can be used to build an influence graph among rules. These features, or the influence graph, can also be used to assess the impact of a change in the rule program: a user who intends to modify some rule will probably want to consider adjusting the rules that are enabled or disabled by the rule about to be modified, or that enable or disable it.

In this respect, a user who is in the process of modifying a rule $r$ into $r^{\prime}$ could be interested in the rules that were enabled by $r$ and no longer are by its modified version $r^{\prime}$. And similarly, in the rules that were not enabled by $r$ but are by $r^{\prime}$, as well as the three other pairs of combinations. This goes one step further than the "may enable" family of features, and introduces a kind of derivative of these features.

\section{A.3.3 Rule may become applicable when...}

This Rule Static Analysis feature is only interested in the fact that a previously inapplicable rule becomes applicable, without focusing on the cause of this state transition, such as a rule execution. This feature thus reports the rules that become applicable as the result of a transition of the system, from a state where some condition does not hold to a state where it holds. 
Example. Consider the rule below:

$$
r(p: \text { Person }): \text { p.bonus }>20 \rightarrow \text { p.cat }:=\text { Gold }
$$

This rule "may become applicable when p.bonus $>30$ " because there exists a transition between two states, in which on the one hand the assertion p.bonus $>$ 30 is initially false and finally true, and on the other hand the rule $r$ is initially not applicable and finally is. An example of such a transition is from a state where the bonus is 10 to a state where it is 40 .

Considering a rule $r=(\vec{o}, \vec{T}, g, a)$ and an assertion $\varphi$ that represents the query, we say that

$$
\text { " } r \text { may become applicable when } \varphi \text { " }
$$

if

there exist two states $s$ and $s^{\prime}$ and a valuation $\nu$, such that

$$
s, \nu \models \neg g \wedge \neg \varphi \quad \text { and } \quad s^{\prime}, \nu \models g \wedge \varphi .
$$

As is visible in the formalization, and illustrated by the example, the cause for the transition is not explicited. It could be the execution of a rule, or of several rules. However it must be the case in the transition, that the query (p.bonus $>30$ in the example above) is initially false and it becomes true. This requirement is explicit in the formalization of the feature, even if not in its wording.

Although apparently close to the "rule may apply when..." feature, the "rule may become applicable when..." feature has a different usage. The "rule may apply when..." feature is used when auditing a rule-based application, in the search for all rules that will be considered for application in a given situation. The "rule may become applicable when..." feature is rather used when extending a rule program, to look for rules that deal with cases similar to the one about to be implemented.

Another way to look at the "rule may become applicable when..." feature is to see it as a "rule may apply when..." feature that would retrieve only the rules that actually involve the attributes that occur in the query. Indeed, a rule can change from not being applicable to being applicable only if its guard is affected by the change in the truth value of the query.

\section{A.4 Redundancy}

\section{A.4.1 Equivalent rules}

This Rule Static Analysis feature detects and reports pairs of rules that are applicable in the same working memories, and that produce the same ones when executed.

Example. The two rules below are "equivalent rules":

$$
\begin{aligned}
& r_{1}(p: \text { Person }): p \cdot \text { age } \geq 21 \rightarrow \text { p.bonus }:=\text { p.bonus } \times 2 \\
& r_{2}(p: \text { Person }): \text { p.age }>20 \rightarrow \text { p.bonus }:=\text { p.bonus }+ \text { p.bonus }
\end{aligned}
$$


Whether or not it is a bug for a rule program to contain two equivalent rules is a decision to be taken by the author of the program. In the example above, removing one of the two equivalent rules will actually change the program behavior, since with both rules the bonus of a matching person is multiplied by 4 , whereas it would be doubled with only one of the two rules.

This feature can be formalized as follows.

$$
r_{1} \text { and } r_{2} \text { are "equivalent rules" }
$$

if

$$
\stackrel{r_{1}}{\longrightarrow} \text { and } \stackrel{r_{2}}{\longrightarrow} \text { are the same relation. }
$$

\section{A.4.2 Redundant rules}

This feature detects and reports pairs of rules in which the first rule makes the second one redundant, that is, each time the second rule is applicable the first is as well, and it produces at least the same effect as the second.

Example. Consider the rules below. (The rule $r_{1}$ includes two assignments in its action, which is not allowed by our rule language. We take this liberty here in order to illustrate the feature.)

$$
\begin{aligned}
& r_{1}(p: \text { Person }): p \cdot \text { age } \geq 20 \rightarrow \text { p.bonus }:=\text { p.bonus }+20 ; \text { p.cat }:=\text { Gold } \\
& r_{2}(p: \text { Person }): \text { p.age }>40 \rightarrow \text { p.bonus }:=\text { p.bonus }+20
\end{aligned}
$$

In this rule program " $r_{1}$ makes $r_{2}$ redundant". Indeed, each time the rule $r_{2}$ is applicable, the rule $r_{1}$ is also applicable; and when $r_{1}$ is executed it performs the same actions as $r_{2}$, and possibly more.

Again, whether or not it is a bug for a rule program to contain redundant rules is a decision to be taken by the author of the program. Here, the question is whether the bonus of persons over 40 should be increased by 20 , as performed by $r_{1}$, or by 40 , which is the behavior of the rule program $\left\{r_{1}, r_{2}\right\}$. In the latter case however, the implementation above with $r_{2}$ performing only the extra work not performed by $r_{1}$, is quite error-prone.

A formalization of this feature in our framework, where only one assignment is allowed in the action of a rule, is that

$$
\text { " } r_{1} \text { makes } r_{2} \text { redundant" }
$$

if

$$
\text { the relation } \stackrel{r_{2}}{\longrightarrow} \text { is included in the relation } \stackrel{r_{1}}{\longrightarrow} \text {. }
$$

In a setting where redundant rules could have distinct actions, a formalization would require a finer definition of how an update is included in another, for example based on an order on states. 


\section{A.5 Confluence and Completeness}

\section{A.5.1 Conflicting rules}

This Rule Static Analysis feature reports two rules as conflicting if they can be applied to a common working memory, and they update a common object attribute with two different values. As discussed further below, this feature does not address the confluence of the whole rule program, which is left out of the current scope of the IBM Websphere Operational Decision Management product.

Example. Consider the rules below:

$$
\begin{aligned}
& r_{1}(p: \text { Person }): \text { p.age } \geq 60 \rightarrow \text { p.bonus }:=15 \\
& r_{2}(p: \text { Person }): \text { p.cat }=\text { Gold } \rightarrow \text { p.bonus }:=20
\end{aligned}
$$

These rules are reported as "conflicting" because when applied to a senior gold person, $r_{1}$ grants a bonus of 15 whereas $r_{2}$ grants 20 .

This feature can be formalized as follows, considering two rules $r_{1}$ and $r_{2}$ defined as $r_{1}=\left(\vec{o}_{1}, \vec{T}_{1}, g_{1}, a_{1}\right)$ and $r_{2}=\left(\vec{o}_{2}, \vec{T}_{2}, g_{2}, a_{2}\right)$, with the notation of actions introduced in Section 3.5.3, that is, $a_{1} \equiv o_{1}^{1} \cdot f_{r_{1}}:=e_{r_{1}}$ and $a_{2} \equiv o_{1}^{2} \cdot f_{r_{2}}:=e_{r_{2}}$.

$$
r_{1} \text { and } r_{2} \text { are "conflicting" }
$$

if

there exist three states $s, s_{1}^{\prime}$, and $s_{2}^{\prime}$, and a valuation $\nu$, such that

$$
\begin{aligned}
& \nu\left(o_{1}^{1}\right)=\nu\left(o_{1}^{2}\right) \quad \text { and } \quad f_{r_{1}} \text { is the same as } f_{r_{2}} \quad \text { and } \quad s, \nu \models g_{1} \wedge g_{2} \\
& \text { and } \quad s \stackrel{\left(r_{1}, \nu\right)}{\longrightarrow} s_{1}^{\prime} \quad \text { and } \quad s \stackrel{\left(r_{2}, \nu\right)}{\longrightarrow} s_{2}^{\prime} \quad \text { and } \quad s_{1}^{\prime} \neq s_{2}^{\prime} \text {. }
\end{aligned}
$$

Note that the first line in the formalization above is important. A feature implementing only the second line would suffer from the following issues: If not imposing that both rules are applicable, the execution of either rule might reduce to skip, in which case the final states $s_{1}^{\prime}$ and $s_{2}^{\prime}$ would be different just because one rule performed an action while the other left the initial state unchanged. If not imposing that the attribute symbols $f_{r_{1}}$ and $f_{r_{2}}$ are the same, the feature would report as conflicting any pair of rules that update distinct attributes (such as one rule assigning a bonus and another rule updating a category), since their executions actually have different effects. Finally, if not imposing that the actions of the rules are performed on the same object, the feature would report as conflicting any pair of rules that update the same attribute, since an execution of each rule on a different object results in different states.

We can also note that, by the definition of rule execution (see Section 5.3), the second line can be reduced to $s, \nu \models e_{r_{1}} \neq e_{r_{2}}$ (hence eliminating the need for states $s_{1}^{\prime}$ and $s_{2}^{\prime}$ in the formalization).

\section{Confluence}

The "conflicting rules" feature is a first step toward checking the confluence of the rule program. A rule program $\mathcal{R}$ is confluent if for any states $s, s_{1}^{\prime}$, and $s_{2}^{\prime}$, such that $s \stackrel{\mathcal{R}}{\longrightarrow} s_{1}^{\prime}$ and $s \stackrel{\mathcal{R}}{\longrightarrow} s_{2}^{\prime}$ one has $s_{1}^{\prime}=s_{2}^{\prime}$ [BL10]. That is, the relation $\stackrel{\mathcal{R}}{\longrightarrow}$ 
is a (partial) function. However, conflicting rules can exist in a confluent rule program; and furthermore a rule program can be non confluent and contain no conflicting rules. The following two examples illustrate this.

Consider the two rules below, taken from the example discussed in Section 10.1.2. They are conflicting, because when executed on a senior gold person with a zero bonus, the first one assigns a bonus of 15 and the second one assigns a bonus of 20 .

$$
\begin{aligned}
& r_{1}(p: \text { Person }): \text { p.age } \geq 60 \rightarrow \text { p.bonus }:=\text { p.bonus }+15 \\
& r_{2}(p: \text { Person }): \text { p.cat }=\text { Gold } \rightarrow \text { p.bonus }:=\text { p.bonus }+20
\end{aligned}
$$

However, in each case the other rule is applicable, and the complete execution of the program $\left\{r_{1}, r_{2}\right\}$ ends with a bonus of 35 .

Consider now the rules below. They are not conflicting in the sense of the Rule Static Analysis feature, since they do not update the same attribute.

$$
\begin{aligned}
& r_{1}(p: \text { Person }): \text { p.age } \geq 60 \rightarrow \text { p.cat }:=\text { Platinum } \\
& r_{2}(p: \text { Person }): \text { p.cat }=\text { Gold } \rightarrow \text { p.bonus }:=10
\end{aligned}
$$

However they form a non-confluent program. For example, on a senior gold person with a zero bonus, both rules are applicable. The execution in which rule $r_{1}$ is applied first ends after this application with a zero bonus and a Platinum category. In contrast, the execution where $r_{2}$ is first applied sets the bonus to 10, then applies $r_{1}$ to update the category.

\section{A.5.2 Completeness analysis}

This Rule Static Analysis feature reports cases for which a rule is suspected to be missing.

Example. Consider the rule program made of the rules below:

$$
\begin{aligned}
& r_{1}(p: \text { Person }): \text { p.age } \leq 20 \text { or p.age } \geq 60 \rightarrow \text { p.bonus }:=10 \\
& r_{2}(p: \text { Person }): \text { p.cat } \neq \text { Gold } \rightarrow \text { p.bonus }:=20
\end{aligned}
$$

This program contains no rule to address the case where the person's age is between 20 and 60 and their category is Gold. Whether or not this is a bug is the decision of the author of the program, as it might be the case that for gold persons between 20 and 60 , the bonus should be left unchanged.

Roughly speaking, a rule program is incomplete when there exists a working memory in which no rule is applicable.

However, this too simple formalization has to be refined to produce reasonable results. For example, an empty working memory would cause no rule to be applicable. Which leads to the question of which sets of instances of each type should be considered to get meaningful working memories. As another example of the subject complexity, adding the rule $r_{3}$ below to the rule program above is usually not considered as making it complete, since even though there are now applicable rules for all kinds of persons, the rule $r_{3}$ can be seen as unrelated to the rules $r_{1}$ and $r_{2}$ because they update distinct attributes.

$$
r_{3}(p: \text { Person }): \text { p.cat }=\text { Gold } \rightarrow \text { p.cat }:=\text { Platinum }
$$


A formal definition of the completeness of a rule program that also matches the expectations of users for Business Rules Management Systems is the subject of the work of Ulrich Junker, as well as methods to detect and report missing cases [Jun11].

\section{A.6 Safety}

\section{A.6.1 Rule may have an unsafe execution}

This Rule Static Analysis feature is a particular case of safety property checking. It is based on additional information provided by the user, in the form of properties of attributes that are used as invariants. The "unsafe execution" feature reports rules whose execution can violate these invariants.

Although one might imagine more general forms, the feature limits invariants provided by the user to closed assertions of the form $\forall 0: T \varphi$, where is a formula in the sole variable $o$ and involving only one attribute $f$ defined on the type $T$.

Example. Consider a setting where the following two invariants have been stated by the user:

$$
\forall p: \text { Person }(p . a g e \geq 0) \quad \forall c: \text { Cart }(c . \text { discount } \in[0,100]) .
$$

Consider now the rule below:

$$
\mathrm{r}_{1}(\mathrm{p}: \text { Person, } \mathrm{c}: \text { Cart }): \text { p.cat }=\text { Gold } \& \mathrm{c}=\text { p.cart } \rightarrow \text { c.discount }:=\mathrm{p} \text {.age }
$$

When executed on a person aged more than 100 (which is allowed by the invariant on the age of persons), this rule will set the discount of the cart to a value forbidden by the invariant on discounts.

The feature can be formalized as follows. Let us note $I$ the conjunction of all the invariants provided by the user. Given a rule $r$, we say that

$$
\text { " } r \text { may have an unsafe execution" }
$$

if

there exist two states $s$ and $s^{\prime}$ and a valuation $\nu$, such that

$$
s \models I \quad \text { and } \quad s \stackrel{(r, \nu)}{\longrightarrow} s^{\prime} \quad \text { and } \quad s^{\prime} \not \models I .
$$

That is, if

$$
\{I\} r\{I\} \quad \text { does not hold. }
$$

This feature can be seen as the restriction to one rule of the verification of $I$ as an invariant of the rule program, that is, $\{I\} \mathcal{R}\{I\}$. It is obvious that if no rule "may have an unsafe execution", then $I$ is an invariant of the rule program. This can be proven, for example, with proof rule (PR1). However, the opposite is not true. That is, it can happen that $I$ is an invariant of the rule program even if some rule "may have an unsafe execution". Consider for instance the rule program $\mathcal{R}=\left\{r_{1}, r_{2}\right\}$ made of the rule $r_{1}$ above and the rule $r_{2}$ below:

$$
\mathrm{r}_{2}(\mathrm{c}: \text { Cart }): \text { c.discount }>100 \rightarrow \text { c.discount }:=100
$$


Let us note $I \equiv \forall p:$ Person $(p$. age $\geq 0) \wedge \forall c$ : Cart (c.discount $\in[0,100])$. As seen in the example, the correctness formula $\{I\} r_{1}\{I\}$ does not hold. However the correctness formula $\{I\} \mathcal{R}\{I\}$ holds, as proven in Section 10.1.1 on a similar rule program.

The "rule may have an unsafe execution" feature is a specialized form of safety property checking. The general form could be expressed with a correctness formula as defined in Chapter 6 , and verified with the method described in Part III.

\section{A.7 Discussion}

The Rule Static Analysis features described in this chapter were developed without the formal framework introduced in this thesis. Therefore, they may not be considered as a result of the thesis. Rather, their development created the need for such a formal framework, and initiated its elaboration.

A posteriori, the fact that they can be expressed quite simply within our formal framework demonstrates that this framework is well-suited to model rule programs as handled by Business Rule Management Systems, as well as their semantics and their execution properties. It gives a positive indication on its ability to support the development of further verification features.

As mentioned previously, all the Rule Static Analysis features in the IBM Websphere Operational Decision Management product are limited to at most one rule execution. Some features, such as the "conflicting rules" detection, consider the execution of two rules, but these executions are only considered concurrently, as opposed to being chained. In this, the IBM Websphere Operational Decision Management product is not weaker than other commercial BRMS products, which sometimes do not even go beyond a syntax-based analysis, failing for instance to detect that the two rules given as an example for the "equivalent rules" feature are actually equivalent.

However this limitation to one rule transition motivated our search for a verification method to step from the scope of one rule to the scope of a whole rule program. Or, as we put it in Chapter 11, to resolve the tension between the local and global approaches to rule programs in BRMS.

The area where our work can immediately contribute to the development of new features, is the expression of safety properties. Chapter 6 provides a definition for rules and rule programs of the classic $\{p\} S\{q\}$ formalism for safety properties. BRMS can thus readily implement the tools to express specifications that a rule program should comply with, although another obstacle will be the user adoption due to the additional work implied to express these specifications.

Based on these user-provided specifications, BRMS could also implement features related to the testing for a rule program, as mentioned in Section 1.2. This would include checking that the expected output for each test input conforms with the specification; assessing the comprehensiveness of the data sets used as input of the tests, with respect to the data space that the program might receive as input; or proposing new test cases to enhance this coverage. Other features proposed in Section 1.2, such as run-time or historical testing, could also benefit from the explicitation of specifications.

As an application of Part III, BRMS could implement the methods for proving a global correctness property of a rule program out of individual properties 
of the rules. This would require either to devise an algorithm to exploit the proof rules as illustrated in Sections 8.4, 9.5, and 10.1.2, or to embed an interactive theorem prover in order to achieve the proofs with the assistance of the user. 


\section{List of Figures}

1.1 A business rule written with IBM Websphere Operational Decision Management ............... 5

3.1 Grammar - Expressions and formulas . . . . . . . . . . . . . 24

3.2 Grammar - Expressions and formulas on numbers and symbolic values (extension to Fig. 3.1) . . . . . . . . . . . . . 26

3.3 Grammar - Assignment . . . . . . . . . . . . . 27

5.1 Execution of a rule program by a rule engine . . . . . . . . . . . 47

5.2 Example - Attribute span, $\mathcal{T}$-compliant state, type instances . . 51

5.3 Automaton - Identity Eligibility Strategy . . . . . . . . . . . . . 64

5.4 Automaton - Refraction Eligibility Strategy . . . . . . . . . . 66

5.5 Automaton — Sequential Eligibility Strategy . . . . . . . . . 68

5.6 Automaton - One-Shot Eligibility Strategy . . . . . . . . . . 68

6.1 Grammar — Loop-free programs . . . . . . . . . . . . . 78

7.1 Grammar — Parallel programs with loop-free processes . . . . . 88

7.2 Grammar - Expressions and assignment with ghost variables . . 94

7.3 Grammar - while Programs . . . . . . . . . . . . . . . . . 95

7.4 Grammar - Parallel programs composed of while programs . . 95

7.5 Grammar - Nondeterministic programs . . . . . . . . . . . . 102

8.1 Example - Bonus depending on the age . . . . . . . . . . . 118

10.1 A decision table and its implementation as a rule program . . . . 171 



\section{List of Proof Rules}

\section{General Proof Rule}

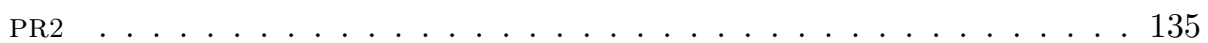

Soundness: Theorem 9.2 on page 135 .

Completeness: Theorem 9.4 on page 140.

\section{Specialized Proof Rules}

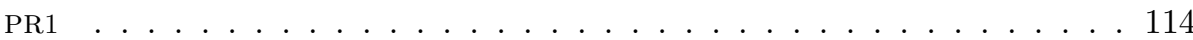

Unary rules; eligibility does not have to be taken into account.

Soundness: Theorem 8.2 on page 114 .

Incompleteness: Theorem 8.5 on page 122 .

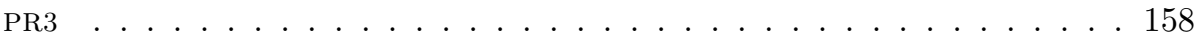

Unary rules.

Soundness and completeness: Theorem 10.2 on page 158 .

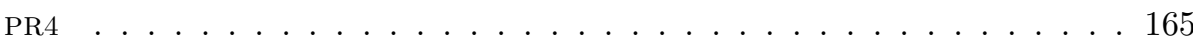

Working memory contains at most one instance of each type.

Soundness and completeness: Theorem 10.5 on page 165 .

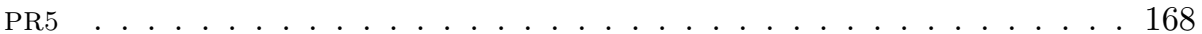

Rule program is disjoint from the correctness formulas for the rules.

Soundness: Theorem 10.6 on page 168.

PR6

Rule program contains a single, unary rule.

PR7

Two disjoint rule programs.

Soundness: Theorem 10.7 on page 170 . 



\section{Bibliography}

[AdBO09] Krzysztof R. Apt, Frank S. de Boer, and Ernst-Rüdiger Olderog. Verification of Sequential and Concurrent Programs. Texts in Computer Science. Springer-Verlag, 3rd edition, 2009. Cited on pages $8,17,41,44,74,80,82,88,100,110,111,114$, and 167 .

$\left[\mathrm{ADG}^{+} 05\right]$ Grigoris Antoniou, Carlos Viegas Damásio, Benjamin Grosof, Ian Horrocks, Michael Kifer, Jan Maluszynski, and Peter F. Patel-Schneider. Combining rules and ontologies: a survey. Technical Report IST506779/Linköping/I3-D3/D/PU/a1, Linköping University, February 2005. IST-2004-506779 REWERSE Deliverable 2005-I3-D3, http://rewerse.net/publications/ rewerse-description/REWERSE-DEL-2005-I3-D3.html.

Cited on pages 12 and 13.

[AK07] Hassan Aït-Kaci. Description logic vs. order-sorted feature logic. In Diego Calvanese, Enrico Franconi, Volker Haarslev, Domenico Lembo, Boris Motik, Anni-Yasmin Turhan, and Sergio Tessaris, editors, Description Logics, volume 250 of CEUR Workshop Proceedings. CEUR-WS.org, 2007. http://ceur-ws.org/Vol-250/ paper_2.pdf.

Cited on page 14.

$\left[\mathrm{AKBJ}^{+} 07\right]$ Hassan Ait-Kaci, Bruno Berstel, Ulrich Junker, Michel Leconte, and Andreas Podelski. Satisfiability modulo structures as constraint satisfaction: an introduction. In Pierre-Étienne Moreau and Sandrine Blazy, editors, Dix-huitièmes Journées Francophones des Langages Applicatifs, 2007. http://jfla.inria.fr/2007/.

Cited on page 180.

[AKL09] Jürgen Angele, Michael Kifer, and Georg Lausen. Ontologies in Flogic. In Peter Bernus, Jacek Blazewics, Günter Schmidt, Michael Shaw, Steffen Staab, and Rudi Studer, editors, Handbook on Ontologies, International Handbooks on Information Systems, pages 45-70. Springer, 2009.

Cited on page 14.

[AKPCG97] Hassan Aït-Kaci, Andreas Podelski, and Seth Copen Goldstein. Order sorted feature theory unification. Journal of Logic Programming, 30(2):99-124, 1997.

Cited on page 14. 
[Alb94] Patrick Albert. ILOG Rules, embedding rules in C++: Results and limits. In Proceedings of the OOPSLA'94 Workshop on Embedded Object-Oriented Production Systems (EOOPS), 1994.

Cited on page 3 .

[ALS90] Milam W. Aiken and Olivia R. Liu Sheng. Nexpert Object. Expert Systems, 7(1):54-57, 1990.

Cited on pages 3 and 4.

[AO97] Krzysztof R. Apt and Ernst-Rüdiger Olderog. Verification of Sequential and Concurrent Programs. Texts in Computer Science. Springer-Verlag, 2nd edition, 1997.

Cited on pages 8 and 110.

[Apt81] Krzysztof R. Apt. Ten years of Hoare's logic: A surveypart I. ACM Transactions on Programming Languages and Systems, 3(4):431-483, 1981.

Cited on pages 16, 74, 75, 76, 80, and 82 .

[Apt84] Krzysztof R. Apt. Ten years of Hoare's logic: A survey-part II: Nondeterminism. Theoretical Computer Science, 28:83-109, 1984.

Cited on pages 16, 74, 87, 105, 140, and 142.

[Ash75] Edward A. Ashcroft. Proving assertions about parallel programs. Journal of Computer and System Sciences, 10(1):110-135, 1975.

Cited on page 17.

[AV88] Serge Abiteboul and Victor Vianu. Procedural and declarative database update languages. In PODS, pages 240-250. ACM, 1988. Cited on page 14.

[AV91] Serge Abiteboul and Victor Vianu. Datalog extensions for database queries and updates. Journal of Computer and System Sciences, 43(1):62-124, 1991.

Cited on page 14.

$\left[\mathrm{BBB}^{+} 07\right]$ Bruno Berstel, Philippe Bonnard, François Bry, Michael Eckert, and Paula-Lavinia Pătrânjan. Reactive rules on the web. In Grigoris Antoniou, Uwe Aßmann, Cristina Baroglio, Stefan Decker, Nicola Henze, Paula-Lavinia Pătrânjan, and Robert Tolksdorf, editors, Reasoning Web, volume 4636 of Lecture Notes in Computer Science, pages 183-239. Springer, 2007.

Cited on page 13.

$\left[\mathrm{BCM}^{+}\right.$03] Franz Baader, Diego Calvanese, Deborah L. McGuinness, Daniele Nardi, and Peter F. Patel-Schneider, editors. The Description Logic Handbook: Theory, Implementation, and Applications. Cambridge University Press, 2003.

Cited on page 14.

[BDS12] Bruno Berstel-Da Silva. Formalizing both refraction-based and sequential executions of production rule programs. In Antonis Bikakis and Adrian Giurca, editors, Rules on the Web: Research 
and Applications, volume 7438 of Lecture Notes in Computer Science, pages 47-61. Springer Berlin / Heidelberg, 2012.

Cited on pages 29, 44, 62, and 64 .

[BFKM85] Lee Brownston, Robert Farrell, Elaine Kant, and Nancy Martin. Programming Expert Systems in OPS5: An Introduction to RuleBased Programming. Addison-Wesley, Boston, USA, 1985.

Cited on pages 2, 45, and 62 .

$\left[\mathrm{BFM}^{+} 06\right]$ Erik Behrends, Oliver Fritzen, Wolfgang May, Franz Schenk, and Daniel Schubert. A framework and components for ECA rules in the web. In Proceedings of Second International Conference on Rules and Rule Markup Languages for the Semantic Web, Athens, Georgia, USA (RuleML), 2006. http://rewerse.net/publications/ rewerse-description/REWERSE-RP-2006-165.html.

Cited on page 13.

[BL07] Chitta Baral and Jorge Lobo. Characterizing production systems using logic programming and situation calculus. http://cs.utep. edu/chitta/papers/char-prod-systems.ps, 2007.

Cited on page 14.

[BL10] Bruno Berstel and Michel Leconte. Using constraints to verify properties of rule programs. In Proceedings of the 2010 Third International Conference on Software Testing, Verification, and Validation Workshops, ICSTW'10, pages 349-354. IEEE Computer Society, 2010.

Cited on pages 16, 180, and 189.

[BLH01] Tim Berners-Lee and James Hendler. The semantic web. Scientific American, 284:34-43, 2001.

Cited on page 12.

[BMWK09] Gérard Basler, Michele Mazzucchi, Thomas Wahl, and Daniel Kroening. Symbolic counter abstraction for concurrent software. In Ahmed Bouajjani and Oded Maler, editors, $C A V$, volume 5643 of Lecture Notes in Computer Science, pages 64-78. Springer, 2009. Cited on page 174.

[BO01] Manfred Broy and Ernst-Rüdiger Olderog. Trace-oriented models of concurrency. In Jan A. Bergstra, Alban Ponse, and Scott A. Smolka, editors, Handbook of Process Algebra, pages 101-195. Elsevier Science B.V., 2001.

Cited on page 17.

[Bou08] Ahmed Bouajjani. Personal communication, 2008.

Cited on page 63.

[BW94] Elena Baralis and Jennifer Widom. An algebraic approach to rule analysis in expert database systems. In Jorge B. Bocca, Matthias Jarke, and Carlo Zaniolo, editors, $V L D B$, pages 475-486. Morgan Kaufmann, 1994.

Cited on pages 14 and 16. 
[CAC11] Amina Chniti, Patrick Albert, and Jean Charlet. Gestion de la cohérence des règles métier éditées à partir d'ontologies OWL. In Conférence Francophone d'Apprentissage 2011, 2011.

Cited on page 15.

[CDAC10] Amina Chniti, Sylvain Dehors, Patrick Albert, and Jean Charlet. Authoring business rules grounded in OWL ontologies. In Mike Dean, John Hall, Antonino Rotolo, and Said Tabet, editors, RuleML, volume 6403 of Lecture Notes in Computer Science, pages 297-304. Springer, 2010.

Cited on page 14 .

[CKMM04] Horatiu Cirstea, Claude Kirchner, Michael Moossen, and PierreÉtienne Moreau. Production systems and Rete algorithm formalisation. Research report, LORIA, Nancy, September 2004. http: //hal.inria.fr/inria-00280938/PDF/rete.formalisation.pdf. Cited on pages 14, 33, 45, and 64 .

[CL08] Diego Calvanese and Georg Lausen, editors. Web Reasoning and Rule Systems, Second International Conference, RR 2008, Karlsruhe, Germany, October 31-November 1, 2008. Proceedings, volume 5341 of Lecture Notes in Computer Science. Springer, 2008. Cited on page 12.

[Coo78] Stephen A. Cook. Soundness and completeness of an axiom system for program verification. Society for Industrial and Applied Mathematics Journal on Computing, 7(1):70-90, 1978.

Cited on page 139.

[CPR07] Byron Cook, Andreas Podelski, and Andrey Rybalchenko. Proving thread termination. In Jeanne Ferrante and Kathryn S. McKinley, editors, PLDI, pages 320-330. ACM, 2007.

Cited on page 176.

[CR03] Chris Culbert and Gary Riley. CLIPS Basic Programming Guide, June 2003.

Cited on page 3.

[CWZ90] David R. Chase, Mark N. Wegman, and F. Kenneth Zadeck. Analysis of pointers and structures (with retrospective). In Kathryn S. McKinley, editor, Best of PLDI, pages 343-359. ACM, 1990.

Cited on page 177.

[dBdBZ80] Jacobus W. de Bakker, Arie de Bruin, and Jeffrey Zucker. Mathematical Theory of Program Correctness. Prentice-Hall international series in computer science. Prentice Hall, 1980.

Cited on pages 16 and 74 .

[dBR09] Jos de Bruijn and Martín Rezk. A logic based approach to the static analysis of production systems. In Axel Polleres and Terrance Swift, editors, RR, volume 5837 of Lecture Notes in Computer Science, pages 254-268. Springer, 2009.

Cited on page 14. 
[Dij75] Edsger W. Dijkstra. Guarded commands, nondeterminacy and formal derivation of programs. Communications of the ACM, 18(8):453-457, 1975.

Cited on pages 101, 102, and 140.

[DL07] Birgit Demuth and Hans-Bernhard Liebau. An approach for bridging the gap between business rules and the semantic web. In Proceedings of the 2007 international conference on Advances in rule interchange and applications, RuleML'07, pages 119-133. SpringerVerlag, 2007.

Cited on page 12.

[dSMHP10] Christian de Sainte Marie, Gary Hallmark, and Adrian Paschke. Rule Interchange Format, Production Rule Dialect. Recommendation, W3C, 2010. http://www.w3.org/TR/rif-prd/.

Cited on pages 3, 14, 33, 45, and 64 .

[ET99] E. Allen Emerson and Richard J. Trefler. From asymmetry to full symmetry: New techniques for symmetry reduction in model checking. In Laurence Pierre and Thomas Kropf, editors, CHARME, volume 1703 of Lecture Notes in Computer Science, pages 142-156. Springer, 1999.

Cited on pages 174 and 177.

[Fai07] Fair, Isaac, and Company. High-volume batch processing with Blaze Advisor. Computer World U.K., March 2007. http://www. computerworlduk. com/white-paper/business-process/ 5092/high-volume-batch-processing-with-blaze-advisor/.

Cited on pages 4, 13, 67, and 176 .

[FH03] Ernest J. Friedman-Hill. JESS in Action. Manning Publications Co., 2003.

Cited on page 3 .

[FHK ${ }^{+}$97] Jürgen Frohn, Rainer Himmeröder, Paul-Thomas Kandzia, Georg Lausen, and Christian Schlepphorst. FLORID: A prototype for F-logic. In W. A. Gray and Per-Åke Larson, editors, ICDE, page 583. IEEE Computer Society, 1997.

Cited on page 13.

[FL92] François Fages and Rémi Lissajoux. Sémantique opérationnelle et compilation des systèmes de production. Revue d'Intelligence Artificielle, 6(4):431-456, 1992.

Cited on pages 14, 33, 45, and 64.

[Flo61] Robert W. Floyd. A descriptive language for symbol manipulation. Journal of the ACM, 8(4):579-584, 1961.

Cited on page 2.

[Flo67] Robert W. Floyd. Assigning meaning to programs. In Proceedings of the Symposium on Applied Math, volume 19, pages 19-32. American Mathematical Society, 1967.

Cited on pages 16 and 74 . 
[For81] Charles Forgy. OPS5 User's manual. Technical Report CMU-CS81-135, Carnegie-Mellon University, Pittsburgh, July 1981.

Cited on pages 2 and 64 .

[For82] Charles Forgy. Rete: A fast algorithm for the many patterns/many objects match problem. Artificial Intelligence, 19(1):17-37, 1982. Cited on pages 3, 12, and 64 .

[GM77] Hervé Gallaire and Jack Minker, editors. Symposium on Logic and Data Bases, Centre d'études et de recherches de Toulouse, Advances in Data Base Theory. Plemum Press, 1977.

Cited on page 14 .

[GNT04] Malik Ghallab, Dana S. Nau, and Paolo Traverso. Automated Planning: Theory and Practice. Morgan Kaufmann, May 2004.

Cited on page 13.

[GS97] Susanne Graf and Hassen Saïdi. Construction of abstract state graphs with PVS. In Orna Grumberg, editor, $C A V$, volume 1254 of Lecture Notes in Computer Science, pages 72-83. Springer, 1997. Cited on page 177.

[GS00] Rik Gerrits and Silvie Spreeuwenberg. VALENS: A knowledge based tool to validate and verify an Aion knowledge base. In Werner Horn, editor, ECAI, pages 731-738. IOS Press, 2000.

Cited on page 15.

[HdBR $\left.{ }^{+} 09\right]$ Stijn Heymans, Jos de Bruijn, Martín Rezk, Hassan Aït-Kaci, Hugues Citeau, Roman Korf, Jörg Pührer, Cristina Feier, and Thomas Eiter. Initial combinations of rules and ontologies. Public Deliverable 3.2, Ontorule Project, December 2009. http: //ontorule-project. eu/outcomes? func=fileinfo\&id $=18$.

Cited on page 14.

[Hen06] Stephen D. Hendrick. Business Rule Management Systems: Addressing Referential Rule Integrity. IDC, 2006. http://www.marketresearch.com/IDC-v2477/ Business-Rule-Management-Systems-Addressing-1286198/. Cited on pages 5 and 7 .

[Hen09] Stephen D. Hendrick. Worldwide Business Rules Management Systems 2009-2013 Forecast. IDC, 2009. http://www. biareports.com/ en/Worldwide_Business_Rules_Management_Systems_2009-2013_ Forecast_A_First_Look_in_a_Difficult_Economy.html. Cited on page 4.

[HH91] Joseph M. Hellerstein and Meichun Hsu. Determinism in partially ordered production systems. Research report, IBM Almaden Research Center, March 1991.

Cited on page 16 .

[HH93] Eric Hanson and Mohammed S. Hasan. Gator: An optimized discrimination network for active database rule condition testing. 
Technical Report TR93-036, University of Florida, 1993.

Cited on page 3.

[Hoa69] C. A. R. Hoare. An axiomatic basis for computer programming. Communications of the ACM, 12:576-580, October 1969.

Cited on pages 16, 74, 76, 78, 80, and 82 .

[Hoa76] C. A. R. Hoare. Parallel programming: An axiomatic approach. Computer Languages, 1(2):151-160, 1976.

Cited on pages 87, 88, and 167.

[HSV99] Masum Hasan, Binay Sugla, and Ramesh Viswanathan. A conceptual framework for network management event correlation and filtering systems. In Sixth IFIP/IEEE International Symposium on Integrated Network Management, pages 233-246, May 1999.

Cited on page 3.

[IBM11] IBM. IBM Websphere Operational Decision Management v7.5 User's Manual, 2011. http://publib.boulder.ibm.com/infocenter/ dmanager/v7r5/.

Cited on pages 4, 13, 67, 176, and 179 .

[JM79] Neil D. Jones and Steven S. Muchnick. Flow analysis and optimization of LISP-like structures. In Proceedings of the 6th ACM SIGACT-SIGPLAN symposium on Principles of programming languages, POPL '79, pages 244-256, New York, NY, USA, 1979. ACM.

Cited on page 177.

[Jun11] Ulrich Junker. Satisfiability techniques for detecting missing cases in incomplete business rule projects. U.S. Patent 20110082826, IBM, July 2011. http://www.freepatentsonline. com/y2011/0082826.html.

Cited on page 191.

[KB70] Donald Knuth and Peter Bendix. Simple word problems in universal algebra. In Computational Problems in Abstract Algebra, pages 263-297. Pergamon Press, 1970.

Cited on pages 16 and 177.

[KL89] Michael Kifer and Georg Lausen. F-logic: A higher-order language for reasoning about objects, inheritance, and scheme. In Proceedings of the 1989 ACM SIGMOD international conference on Management of data, SIGMOD'89, pages 134-146. ACM, 1989. Cited on page 14.

[KLW95] Michael Kifer, Georg Lausen, and James Wu. Logical foundations of object-oriented and frame-based languages. Journal of the ACM, 42(4):741-843, 1995.

Cited on page 25.

[Knu03] Donald E. Knuth. Robert W. Floyd, in memoriam. SIGACT News, 34(4):3-13, 2003. http://courses.csail.mit.edu/6.042/spring10/ 
floyd-eulogy-by-knuth.pdf.

Cited on page 2.

[KSS87] Robert A. Kowalski, Fariba Sadri, and Paul Soper. Integrity checking in deductive databases. In Proceedings of the 13th International Conference on Very Large Data Bases, VLDB'87, pages 61-69. Morgan Kaufmann Publishers Inc., 1987.

Cited on page 15.

[KU94] Anton P. Karadimce and Susan D. Urban. Conditional term rewriting as a formal basis for active database rules. In $R I D E-A D S$, pages 156-162, 1994.

Cited on page 16 .

[Lam77] Leslie Lamport. Proving the correctness of multiprocess programs. IEEE Transactions on Software Engineering, 3(2):125-143, 1977. Cited on pages 17 and 109.

[Lam93] Leslie Lamport. Verification and specification of concurrent programs. In J. W. de Bakker, Willem P. de Roever, and Grzegorz Rozenberg, editors, REX School/Symposium, volume 803 of Lecture Notes in Computer Science, pages 347-374. Springer, 1993. Cited on page 17 .

[Lam94] Leslie Lamport. The temporal logic of actions. ACM Transactions on Programing Languages and Systems, 16(3):872-923, 1994.

Cited on page 39.

[Lam02] Leslie Lamport. Specifying Systems, The TLA+ Language and Tools for Hardware and Software Engineers. Addison-Wesley, 2002. http://research.microsoft.com/en-us/um/people/lamport/ tla/book.html.

Cited on page 17.

[LB06] Michel Leconte and Bruno Berstel. Extending a CP solver with congruences as domains for software verification. In Benjamin Blanc, Arnaud Gotlieb, and Claude Michel, editors, Proceedings of the First Workshop on Constraints in Software Testing, Verification, and Analysis, pages 22-33. IEEE Computer Society Press, 2006.

Cited on page 180.

[LBC03] Shuvendu K. Lahiri, Randal E. Bryant, and Byron Cook. A symbolic approach to predicate abstraction. In Warren A. Hunt, Jr and Fabio Somenzi, editors, CAV, volume 2725 of Lecture Notes in Computer Science, pages 141-153. Springer, 2003.

Cited on page 177.

[Lip75] Richard J. Lipton. Reduction: A method of proving properties of parallel programs. Communications of the ACM, 18(12):717-721, December 1975.

Cited on page 109. 
[LL98] Bertram Ludäscher and Georg Lausen. Handling termination in a logical language for active rules. Informatica (Lithuanian Academy of Sciences), 9(1):65-84, 1998.

Cited on page 16.

[LN94] Gerhard Lakemeyer and Bernhard Nebel, editors. Foundation of Knowledge Representation and Reasoning, volume 810 of Lecture Notes in Computer Science. Springer, 1994.

Cited on page 12.

[Lud98] Bertram Ludäscher. Integration of Active and Deductive Database Rules, volume 45 of DISDBIS. Infix Verlag, St. Augustin, Germany, 1998.

Cited on pages 12 and 13.

[Man03] Zohar Manna. Mathematical Theory of Computation. Dover books on mathematics. Dover Publications, 2003.

Cited on page 78.

[Met91] William Mettrey. A comparative evaluation of expert system tools. Computer, 24:19-31, 1991.

Cited on page 62.

[Min96] Jack Minker. Logic and databases: A 20 year retrospective. In Dino Pedreschi and Carlo Zaniolo, editors, Logic in Databases, volume 1154 of Lecture Notes in Computer Science, pages 3-57. Springer, 1996.

Cited on pages 12, 14, and 15 .

[Mir87] Daniel P. Miranker. TREAT: A better match algorithm for AI production systems. In Proceedings of the Sixth National Conference on Artificial Intelligence - Volume 1, AAAI'87, pages 42-47. AAAI Press, 1987.

Cited on page 3.

[Mor82] Joseph M. Morris. A general axiom of assignment. In F. L. Bauer, E. W. Dijkstra, and C. A. R. Hoare, editors, Theoretical Foundations of Programming Methodology: Lecture Notes of an International Summer School. Reidel, 1982.

Cited on pages 17, 41, 80, and 111.

[MP89] Zohar Manna and Amir Pnueli. Completing the temporal picture. In Giorgio Ausiello, Mariangiola Dezani-Ciancaglini, and Simona Ronchi Della Rocca, editors, ICALP, volume 372 of Lecture Notes in Computer Science, pages 534-558. Springer, 1989.

Cited on page 39.

[MP05] Tim Menzies and Charles Pecheur. Verification and validation and artificial intelligence. Advances in Computers, 65:154-203, 2005. Cited on page 15.

[MS06] David W. McCoy and Jim Sinur. Achieving Agility: The Agile Power of Business Rules. Gartner, 2006. http://www.gartner. 
com/DisplayDocument?doc_cd=138218.

Cited on pages 2 and 4 .

[Nak04] Seynabou Diop Nakoulima. Added value of LibRT VALENS for ILOG JRules. Rapport de stage, École des Mines de Nancy, 2004. Available from Bruno Berstel-Da Silva.

Cited on page 15.

[NS72] Allen Newell and Herbert A. Simon. Human Problem Solving. Prentice Hall, Englewood Cliffs, NJ, USA, 1972.

Cited on pages 2 and 12 .

[OG76] Susan S. Owicki and David Gries. An axiomatic proof technique for parallel programs I. Acta Informatica, 6:319-340, 1976. Cited on pages 8, 17, 87, 92, 100, 109, 110,114, and 176 .

[Old84] Ernst-Rüdiger Olderog. Hoare's logic for programs with procedures-What has been achieved? In Edmund M. Clarke and Dexter Kozen, editors, Logics of Programs, volume 164 of Lecture Notes in Computer Science, pages 383-395. Springer, 1984.

Cited on pages 16 and 74 .

[Old91a] Ernst-Rüdiger Olderog. Correctness of concurrent processes. Theoretical Computer Science, 80:263-288, 1991.

Cited on page 17.

[Old91b] Ernst-Rüdiger Olderog. Nets, Terms and Formulas: Three Views of Concurrent Processes and Their Relationship. Cambridge University Press, 1991. Paperback Edition 2005.

Cited on page 17.

[OMG08] The Object Management Group. Semantics of Business Vocabularies and Business Rules (SBVR 1.0), 2008. http://www.omg.org/ spec/SBVR/1.0/.

Cited on pages 3 and 12 .

[OR93] Ernst-Rüdiger Olderog and Stephan Rössig. A case study in transformational design of concurrent systems. In Marie-Claude Gaudel and Jean-Pierre Jouannaud, editors, Theory and Practice of Software Development (TAPSOFT'93), volume 668 of Lecture Notes in Computer Science, pages 90-104. Springer-Verlag, 1993.

Cited on page 17.

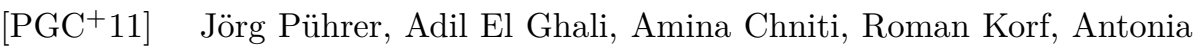
Schwichtenberg, François Lévy, Stijn Heymans, Guohui Xiao, and Thomas Eiter. Consistency maintenance. Public Deliverable 2.3, OnTORULE Project, January 2011. http://ontorule-project.eu/ outcomes func $=$ fileinfo\&id $=44$.

Cited on page 15 .

[PHE10] Jörg Pührer, Stijn Heymans, and Thomas Eiter. Dealing with inconsistency when combining ontologies and rules using DLprograms. In Lora Aroyo, Grigoris Antoniou, Eero Hyvönen, Annette ten Teije, Heiner Stuckenschmidt, Liliana Cabral, and Tania 
Tudorache, editors, ESWC (1), volume 6088 of Lecture Notes in Computer Science, pages 183-197. Springer, 2010.

Cited on page 15.

[Pic07] Gualtiero Piccinini. Allan Newell. In New Dictionary of Scientific Biography. Thomson Gale, 2007. http://www.umsl.edu/ $\sim$ piccininig/Newell $\% 205$.htm.

Cited on pages 2 and 12.

[Plo04] Gordon D. Plotkin. A structural approach to operational semantics. Journal of Logic and Algebraic Programming, 60-61:17-139, 2004. Reprint with corrections of the 1981 article.

Cited on page 57.

[PR04] Andreas Podelski and Andrey Rybalchenko. Transition invariants. In LICS, pages 32-41. IEEE Computer Society, 2004.

Cited on page 176.

[PR05] Andreas Podelski and Andrey Rybalchenko. Transition predicate abstraction and fair termination. In Jens Palsberg and Martín Abadi, editors, POPL, pages 132-144. ACM, 2005.

Cited on page 176.

[PS94] Alun D. Preece and Rajjan Shinghal. Foundation and application of knowledge base verification. International Journal of Intelligent Systems, 9(8):683-701, 1994.

Cited on page 15.

[PSM91] G. Ravi Prakash, Eswaran Subrahmanian, and Hosaker N. Mahabala. A methodology for systematic verification of OPS5-based AI applications. In IJCAI, pages 3-8, 1991.

Cited on page 16.

[PXZ02] Amir Pnueli, Jessie Xu, and Lenore D. Zuck. Liveness with $(0,1, \infty)$-counter abstraction. In Ed Brinksma and Kim Guldstrand Larsen, editors, CAV, volume 2404 of Lecture Notes in Computer Science, pages 107-122. Springer, 2002.

Cited on pages 174 and 177.

[Red10] Red Hat. Drools Expert User Guide, 2010. http: //downloads.jboss.com/drools/docs/5.1.1.34858.FINAL/ drools-expert/html_single/index.html\#d0e2086.

Cited on pages 4, 13, 67, and 176 .

[RG08] John R. Rymer and Mike Gualtieri. The Forrester Wave ${ }^{T M}$ : Business Rules Platforms, Q2 2008. Forrester Research, April 2008. http://www.forrester.com/rb/Research/wave\&trade; _business_rules_platforms, _q2_2008/q/id/39088/t/2.

Cited on page 4.

[RN09] Stuart J. Russell and Peter Norvig. Artificial Intelligence: A Modern Approach. Prentice Hall, Upper Saddle River, New Jersey, 3rd edition, December 2009.

Cited on page 33. 
[Ros03] Ronald Ross. Principles of the Business Rules Approach. AddisonWesley, Boston, 2003.

Cited on pages 3 and 171.

[SA86] Fred B. Schneider and Gregory R. Andrews. Concepts for concurrent programming. In J. W. de Bakker, Willem P. de Roever, and Grzegorz Rozenberg, editors, Current Trends in Concurrency, volume 224 of Lecture Notes in Computer Science, pages 669-716. Springer, 1986.

Cited on page 17.

[SBJ87] Elliot Soloway, Judy Bachant, and Keith Jensen. Assessing the maintainability of XCON-in-RIME: Coping with the problems of a VERY large rule-base. In $A A A I$, pages 824-829, 1987.

Cited on page 6 .

[Sow99] John F. Sowa. Knowledge Representation: Logical, Philosophical, and Computational Foundations. Brooks / Cole, August 1999.

Cited on page 12.

[SS94] James G. Schmolze and Wayne Snyder. Using confluence to control parallel production systems. In Laveen N. Kanal, editor, Parallel Processing for Artificial Intelligence. Elsevier Science Inc., New York, 1994.

Cited on pages 16 and 177.

[SS95] James G. Schmolze and Wayne Snyder. A tool for testing confluence of production rules. In Marc Ayel and Marie-Christine Rousset, editors, EUROVAV, pages 91-104. ADERIAS-LIA, Université de Savoie, 1995.

Cited on page 16.

[SS96] Wayne Snyder and James G. Schmolze. Rewrite semantics for production rule systems: Theory and applications. In Michael A. McRobbie and John K. Slaney, editors, CADE, volume 1104 of Lecture Notes in Computer Science, pages 508-522. Springer, 1996.

Cited on page 16.

[SS97] James G. Schmolze and Wayne Snyder. Detecting redundant production rules. In AAAI/IAAI, pages 417-423, 1997.

Cited on page 16.

[SS99] James G. Schmolze and Wayne Snyder. Detecting redundancy among production rules using term rewrite semantics. KnowledgeBased Systems, 12(1-2):3-11, 1999.

Cited on pages 16 and 177 .

[SSL07] Florian Schmedding, Nour Sawas, and Georg Lausen. Adapting the Rete-algorithm to evaluate F-logic rules. In Adrian Paschke and Yevgen Biletskiy, editors, Advances in Rule Interchange and Applications, volume 4824 of Lecture Notes in Computer Science, pages 166-173. Springer, Berlin, Heidelberg, 2007.

Cited on page 13. 
[SSS82] Motoi Suwa, A. Carlisle Scott, and Edward H. Shortliffe. An approach to verifying completeness and consistency in a rule-based expert system. AI Magazine, 3(4):16-21, 1982.

Cited on page 15.

[TR07] James Taylor and Neil Raden. Smart (enough) systems: How to deliver competitive advantage by automating the decisions hidden in your business. Prentice Hall, Upper Saddle River, NJ, USA, 2007.

Cited on page 3 .

[Ull88] Jeffrey D. Ullman. Principles of Database and Knowledge-Base Systems, Volume I. Computer Science Press, 1988.

Cited on page 14.

[Urq09] Alasdair Urquhart. Emil Post. In Dov M. Gabbay and John Woods, editors, Logic from Russell to Church, volume 5 of Handbook of the History of Logic, pages 617-666. North-Holland, 2009. Cited on page 2.

[vH02] Barbara von Halle. Business Rules Applied. Wiley, New York, 2002.

Cited on page 3.

[vHG06] Barbara von Halle and Larry Goldberg. The Business Rule Revolution. Happy About, 2006.

Cited on page 3.

[Via97] Victor Vianu. Rule-based languages. Annals of Mathematics and Artificial Intelligence, 19(1-2):215-259, 1997.

Cited on pages 11, 13, and 14.

[vLNPS85] Kai von Luck, Bernhard Nebel, Christof Peltason, and Albrecht Schmiedel. BACK to consistency and incompleteness. In Herbert Stoyan, editor, GWAI, volume 118 of Informatik-Fachberichte, pages 245-256. Springer, 1985.

Cited on page 15.

[WC96] Jennifer Widom and Stefano Ceri. Introduction to active database systems. In Active Database Systems: Triggers and Rules For Advanced Database Processing, pages 1-41. Morgan Kaufmann, 1996.

Cited on page 13. 



\section{References to Web Pages}

All URLs below were successfully accessed on October 17, 2012.

[1] Apple. Introducing iPad. http://movies.apple.com/media/us/ipad/2010/ tours/apple-ipad-video-us-20100127_r640-9cie.mov.

Cited on page 175.

[2] The Business Rules Group. http://www.businessrulesgroup.org.

Cited on page 3 .

[3] Corticon Business Rules Management System. http://www. corticon.com/ Products/Business-Rules-Management-System.php.

Cited on page 4.

[4] FICO $^{\text {TM }}$ Blaze Advisor $\AA$. http://www.fico.com/en/Products/DMTools/ Pages/FICO-Blaze-Advisor-System . aspx.

Cited on page 4.

[5] IBM Websphere Operational Decision Management. http://www.ibm.com/ software/websphere/products/business-rule-management.

Cited on pages 4 and 179.

[6] IBM. VISA Europe: Processing payments with unprecedented agility and reliability. http://www.ibm.com/software/success/cssdb.nsf/ CS/SSAO-8FNJEB.

Cited on page 6 .

[7] Ontorule Project. http://ontorule-project.eu.

Cited on page 14.

[8] Oracle Business Rules. http://www.oracle.com/technetwork/middleware/ business-rules/overview.

Cited on page 4.

[9] Red Hat. JBoss Enterprise BRMS. http://www.jboss.com/products/ platforms/brms.

Cited on page 4.

[10] REWERSE Network of excellence. http://rewerse.net.

Cited on page 13.

[11] SAP NetWeaver Business Rules Management. http://www.sap.com/ platform/netweaver/components/brm/index. epx.

Cited on page 4. 



\section{Index}

Page numbers in sans-serif face (as in 66) point to a definition. Page numbers in bold face (as in 66) indicate a result, such as a lemma or a theorem.

A

abstract execution machine, 57, 175

configuration, 57, 63

consistent with unary correctness formulas, 115, 115, 117, 133

final, 74, 100, 132, 135, 139, 147

initial, 58, 59, 132, 135, 145, 147

transition rule, 58, 59

for the one-shot strategy, 74, 116

for the one-shot strategy, with eligibility ghost variables, 131, 136

transition sequence, 59

abstraction

counter, 147, 174, 177

predicate, 177

agenda, 48

head of, see selection

aggregation, 174, see abstraction, counter

aliasing, 17, 41, 111, 112, 129, 147, 151, 174

interference, see interference, due to aliasing

applicability, 7, 13, 45, 47, 53, 58, 100, 128, 181

features in IBM Websphere Operational Decision Management, 181-187

assertion

as pre- or postcondition, see correctness formula

eligibility-aware, see eligibility-aware, assertion

on a state, see state assertion

on a transition between states, see transition assertion

unary, see state assertion, unary

assignment, 27, 29, 44, 78, 168

proof rule for, 80

semantics, 41, 42, 52

well-typed, 27

with ghost variables, 93

attribute

reference, 23, 34

span, see object, attribute span

symbol, 22, 39, 40 
domain, 34, 49, 50

domain is finite, $34,36,50$

in expression or formula, 24

interpretation, 31, 33

updated in rule, 167

update, 41, 44, see assignment

B

business rule, $1,3,12,15,34$

Business Rules Management System, 1, 4

market size, 4

products, $3-4$

IBM Websphere Operational Decision Management, 4, 5, 179-193

\section{C}

collection, see aggregation

completeness, see proof rule, for rule programs, completeness

of a knowledge base, 15

of a rule program, 15, 33

features in IBM Websphere Operational Decision Management, 190-191 relative, 139

conclusion, see proof rule

concurrent program, see parallel program

conflict set, 47,58

resolution strategy, see selection strategy

confluence, 16, 61, 177, 189

features in IBM Websphere Operational Decision Management, 189-190

in active databases, 16

consistency

in a rule program, 15,183

of a knowledge base, 15

constant

interpretation, 33

number, see number

symbol, 22

symbolic value, see symbolic value

correctness formula, $7,8,74$

eligibility-aware, see eligibility-aware, correctness formula

for a rule, 75, 76, 181, 192

focused, $75,75,76$

not focused, 76, 114, 120, 134, $16 \mathbf{1}$

precondition implies postcondition, 147, 163

precondition implies rule guard, 78, 81, 135, 147, 164

well-typed, 75

for a rule program, 82

compared to correctness formula for a rule, $83-85$ 
D

database

active, $13,14,16$

deductive, $12-15,46$

decision table, 171

disjoint

rule and assertions, 168

rule program and assertions, 168,168

rule programs, 168, 170

rules, 90,168

E

ECA rule, see event-condition-action rule

eligibility, $7,13,16,45,47,57,58,63,96,124,126,133,147,158,161,175,180$, 185

eligibility ghost variable, 126, 147, 158, 161, 162

argument list, 126, 134

update on rule execution, see transition assertion, for eligibility ghost variables

eligibility strategy, $47,48,57,58,59,63-69$

as an automaton, 63

identity, 63, 83

one-shot, 68, 73, 96, 103, 126

abstract execution machine transition rule, see abstract execution machine, transition rule, for the one-shot strategy

ensures termination, see termination, ensured by the one-shot strategy

equivalent to refraction on filtering programs, 69

refraction, 64, 64-68, 83

sequential, see execution strategy, sequential

eligibility-aware

abstract execution machine transition rule, see abstract execution machine, transition rule, for the one-shot strategy, with eligibility ghost variables assertion, 127

focused on a rule, 127

selection of disjuncts, 134, 135, 147

correctness formula

for a rule, 131, $\mathbf{1 6 1}$

for a ruleprogram, 132

rule execution, 131

rule instance, 128

application, 129

execution, 129, 130

selectable, 128, 130

event-condition-action rule, $13,16,17$

execution strategy, 46, 48, 58

one-shot, 73

sequential, 4, 13, 64, 67, 67-68, 83, 174, 175

expression, 23

Boolean, see formula, non quantified 
containing ghost variables, 93

interpretation, 33-34

typing, 35

well-typed, 25, 26, 49

$\mathrm{F}$

field, see attribute

first-order logic structure, 31, 32, 37, 40

domain, 32

fixed theory, see theory, fixed

fixed type system, see type system, fixed

formula, 23

closed, 24, 38, 82

containing ghost variables, 93

correctness, see correctness formula

flat, 26, 29, 38, 113

interpretation, 32, 34-36

non quantified, 23, 29

quantified, 23, 36, 38-40

typed, see typed quantification

well-typed, 25, 26, 39, 49, 75

function

ghost variable as, see ghost variable, is a function

operator, 23, 34

uninterpreted, 174, 177

G

gcd in one rule, 103

ghost variable, 93-94, 103, 126, 140

eligibility, see eligibility ghost variable

is a function, 93, 94, see eligibility ghost variable, argument list

shared, 100

ghost-independent state, see state, ghost-independent

guarded command, 101

1

IBM WODM, see Business Rules Management System, products, IBM Websphere Operational Decision Management

ILOG BRMS, see IBM WODM

interference, 29, 85, 109, 110, 167, 175

cross-rule, 111, 114, 133

due to aliasing, 85, 112, 134, 155

avoided by unary rules, 113, 116, 134, 157, 158, 166

avoided by unary working memory, 165, 166

freedom

premises expressing, 76, 114, 120, 123, 133-134, 146, 155

iPad, 175 
$\mathrm{L}$

loop-free program, $78-80$

correctness formula for, 80

derived from a rule, 80,80

proof rules for, 80

$\mathrm{N}$

nondeterministic program, 101, 140-141

derived from a rule program, 103, 140, 141, 142

behaves like the rule program, 104, 142

proof rule for, see proof-rule, DO-OD

semantics

saturated, 46, 102, 140

standard, 102

number, 21, 22, 26, 33, 34, 35, 62

$\mathrm{O}$

object, 17, 21, 32

as instance of a type, see type, instances

attribute span, 49, 49, 50

preservation, $\mathbf{5 2}$

count, see abstraction, counter

equality, 35, 42

tuple, 52,174

well-typed, 55, 175

one-shot

eligibility strategy, see eligibility strategy, one-shot

execution strategy, see execution strategy, one-shot ontology, see semantic web

OPS5, 2, 3, 16, 62

$\mathrm{P}$

parallel program, 17, 95

semantics

interleaving, 88

saturated, 46, 89, 95

verification, 17, 100, 109, 114, 176

with loop-free programs as processes, 88, 91

derived from a rule program, 90, 90

with while programs as processes, 95

cannot simulate rule programs, 99, 110, 114, 176

derived from a rule program, $96, \mathbf{9 7}$

priority, 62

production system, 2, 2, 12, 16, 33, 45

implementations, see Business Rules Management System, products program

loop-free, see loop-free program 
nondeterministic, see nondeterministic program

parallel, see parallel program

rule, see rule program

while, see while program

proof rule, 74

ASSIGNMENT, 80

CONSEQUENCE, 75, 139, 140

DO-OD, 105, 142

for rule programs, $\mathbf{1 1 4}, 118, \mathbf{1 3 5}, \mathbf{1 5 8}, 159, \mathbf{1 6 5}, \mathbf{1 6 8}, \mathbf{1 6 9}, \mathbf{1 7 0}$

completeness, 33, 114, 122, 135, 140, 158, 165, 176

usage, 118-122, 146-155, 159-164, 191

IF, 80

SEQUENCE, 80

SKIP, 80

Q

quantification, see formula, quantified

typed, see typed quantification

$\mathrm{R}$

recency, 62,63

refraction, 63 , see eligibility strategy, refraction

Rete, 3, 12, 13, 64, 175

alternatives, see execution strategy, sequential

rule, 27,180

action, 28, 29, 44, 173

transition assertion, see transition assertion, for a rule action

arity, 28, 62, 112

conflicting, see confluence, features in IBM Websphere Operational Decision Management

disjoint, see disjoint, rules

equivalence and redundancy

features in IBM Websphere Operational Decision Management, 187-188 execution, 56, 76, 81, 181

impact analysis features in IBM Websphere Operational Decision Management, $184-187$

guard, 28, 29, 43, 62, 174, 182, 183

priority, see priority

recency, see recency

specificity, see specificity

type constraint, 28, 29

unary, 112, 157, 158, 166, 168

avoids interference due to aliasing, see interference, due to aliasing, avoided by unary rules

variable types, 28, 29, see variable, naming

variables, 28,28

rule engine, $2,46,93,100$

rule instance, 46, 47, 52, 57, 181 
applicable, see applicability

application, 54, 58, 110, 129, 181

eligible, see eligibility

execution, 55, 121, 129, 181

selectable, see eligibility-aware, rule instance, selectable

well-typed, 55, 58, 59, 117

rule program, 30

abstract execution machine, see abstract execution machine

confluent, see confluence

disjoint, see disjoint, rule programs

execution, 59, 74, 132

final state, see abstract execution machine, configuration, final

strategy, see execution strategy

made of unary rules, see rule, unary

eligibility-aware, $\mathbf{1 5 8}$

operational semantics, 57,175

proof rules for, see proof rule, for rule programs

singleton, 59, 84, 170

S

safety, 16

expressed with a correctness formula, 192

features in IBM Websphere Operational Decision Management, 191-192

selection, 48,58

selection strategy, $47,48,58,59,62,64$

sequential, see execution strategy, sequential

semantic web, $3,12-15$

sequential execution mode, see execution strategy, sequential

sequential program, see while program, see loop-free program

shape analysis, 17,177

signature, 21, 37, 39, 40, 93

specificity, 62,63

state, $32,37,174,180$, see first-order logic structure

consistent with correctness formulas, 136, 136, 138

final, see abstract execution machine, configuration, final

ghost-independent, 97, 141

pair, 40, 41

type-system compliant, 49, 49, 50, 57, 59

preservation, 52, 56, 59

state assertion, 38, 180, see formula

eligibility-aware, see eligibility-aware, assertion

focused on a rule, $39,43,75,113$

global, 38, see formula, closed

unary, 112

structure, see first-order logic structure

symbolic value, 21, 22, 26, 33, 33

equality, 35 
$\mathrm{T}$

term rewriting, 16, 177

termination, $63,64,82, \mathbf{9 7}, 176$

ensured by the one-shot strategy, $\mathbf{6 8}, 74,82$

in a database, 16

in active databases, 16

testing, 6, 192

theory

as extension point, $23,26,27$

fixed, 22, 139, 174

transition assertion, 39-41

backward, 40

for a rule action, 44, 54, 113, 128, 129, 130, 149-152

for eligibility ghost variables, 129, 130, 131, 141, 149-152

for ghost variables, 141

forward, 39

global, 41

identity, 41

transition rule, see abstract execution machine, transition rule type

constraint, 25, 49, 76, 180

domain, 25

for a rule, see rule, type constraint

definition, 25, 166

are disjoint, 25, 50, 166

instances, 50, 50

preservation, $\mathbf{5 2}, \mathbf{5 7}, 59$

name, 22, 25

type system, 25, 49

fixed, 22, 26

state compliant with, see state, type-system compliant

typed quantification, 36, 36, 50, 117

U

unary

assertion, see state assertion, unary

rule, see rule, unary

working memory, see working memory, with one instance of each type

V

valuation, $31,32,180$

and free variables, $\mathbf{3 8}$

and variable names, $\mathbf{3 8}$

well-typed, 43, 51, 56, 76

preservation, 52, 56

variable

bound, 24

free, $24, \mathbf{3 8}, 39,75$ 
ghost, see ghost variable interpretation, see valuation naming, 28-30, 38, 118, 183 scalar, 33

symbol, 22

variable valuation, see valuation

W

while program, 94

working memory, 2, 17, 46, 50, 50, 82, 89, 102, 147, 174, 180 preservation, $\mathbf{5 2}, \mathbf{5 7}, 59$

unary, see working memory, with one instance of each type with one instance of each type, 90, 165, 166 
